\title{
Embracing uncertainty in health technology assessment
}

Citation for published version (APA):

Pouwels, X. G. L. V. (2020). Embracing uncertainty in health technology assessment. [Doctoral Thesis, Maastricht University]. Maastricht University. https://doi.org/10.26481/dis.20201127xp

Document status and date:

Published: 01/01/2020

DOI:

10.26481/dis.20201127xp

Document Version:

Publisher's PDF, also known as Version of record

\section{Please check the document version of this publication:}

- A submitted manuscript is the version of the article upon submission and before peer-review. There can be important differences between the submitted version and the official published version of record.

People interested in the research are advised to contact the author for the final version of the publication, or visit the DOI to the publisher's website.

- The final author version and the galley proof are versions of the publication after peer review.

- The final published version features the final layout of the paper including the volume, issue and page numbers.

Link to publication

\footnotetext{
General rights rights.

- You may freely distribute the URL identifying the publication in the public portal. please follow below link for the End User Agreement:

www.umlib.nl/taverne-license

Take down policy

If you believe that this document breaches copyright please contact us at:

repository@maastrichtuniversity.nl

providing details and we will investigate your claim.
}

Copyright and moral rights for the publications made accessible in the public portal are retained by the authors and/or other copyright owners and it is a condition of accessing publications that users recognise and abide by the legal requirements associated with these

- Users may download and print one copy of any publication from the public portal for the purpose of private study or research.

- You may not further distribute the material or use it for any profit-making activity or commercial gain

If the publication is distributed under the terms of Article $25 \mathrm{fa}$ of the Dutch Copyright Act, indicated by the "Taverne" license above, 


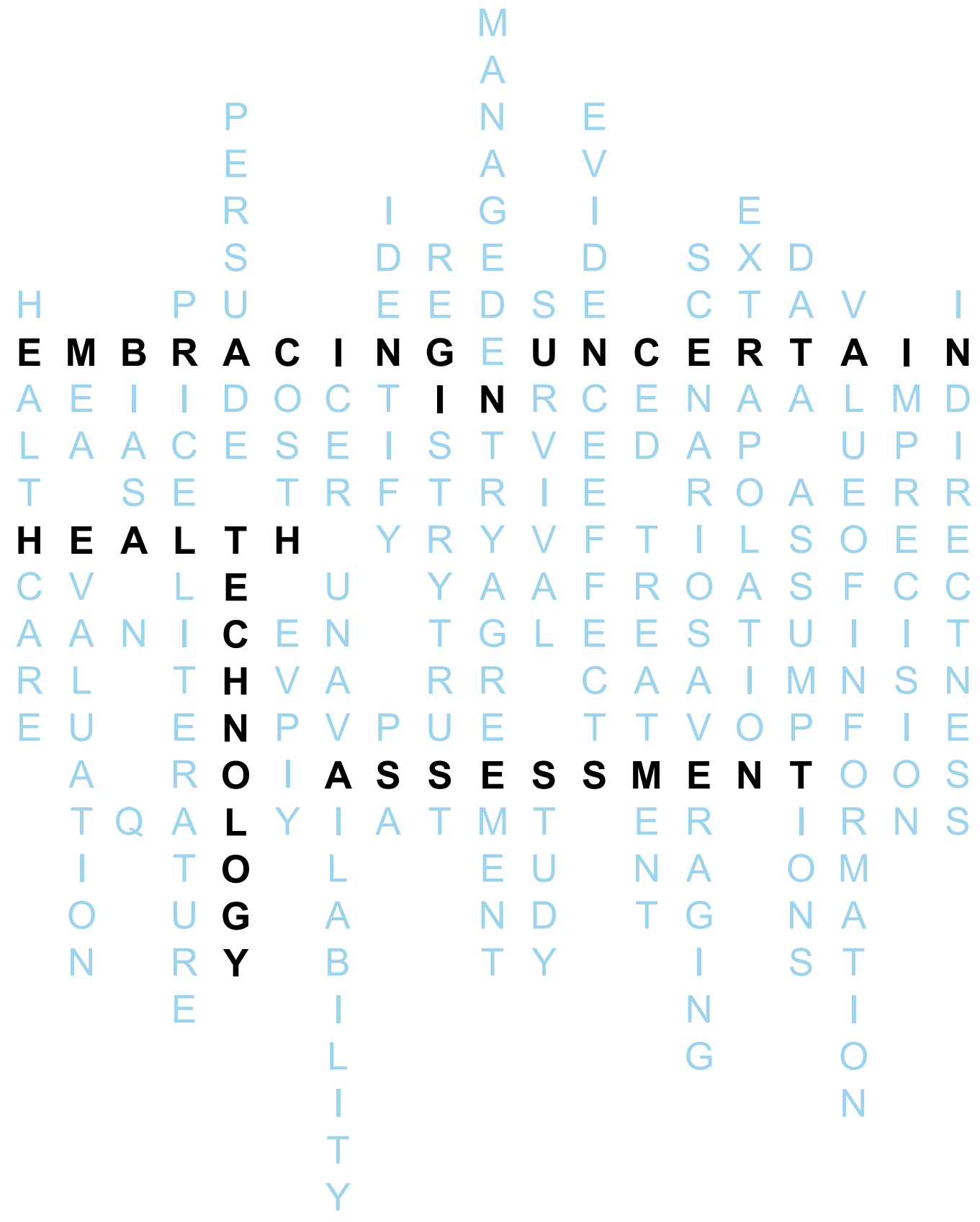

Xavier G.L.V. Pouwels 



\section{Embracing Uncertainty in}

\section{Health Technology Assessment}


C Xavier Ghislain Léon Victor Pouwels, Maastricht 2020.

All rights reserved. No parts of this thesis may be reproduced or transmitted in any form or by any means, without prior permission in writing by the author, or when appropriate, by the publishers of the publications.

Cover: Xavier Pouwels

Lay-out: Tiny Wouters

Production: Gildeprint

ISBN: 978-94-6419-070-0 


\title{
Embracing Uncertainty in
}

\section{Health Technology Assessment}

\author{
PROEFSCHRIFT \\ ter verkrijging van de graad van doctor aan de Universiteit Maastricht, \\ op gezag van de Rector Magnificus, Prof. dr. Rianne M. Letschert \\ volgens het besluit van het College van Decanen, \\ in het openbaar te verdedigen \\ op vrijdag 27 november 2020 om 14.00 uur
}

door

Xavier Ghislain Léon Victor Pouwels 


\section{Promotor}

Prof. dr. M.A. Joore

\section{Co-promotoren}

Dr. B.L.T. Ramaekers

Dr. M. de Boer

\section{Beoordelingscommissie}

Prof. dr. C.D. Dirksen (voorzitter)

Prof. dr. ir. M. B. A. van Asselt

Dr. ir. W.G. Goettsch (Universiteit Utrecht)

Prof. dr. D.K.M. De Ruysscher

Prof. dr. S. Siesling (Universiteit Twente) 


\section{Table of contents}

$\begin{array}{lll}\text { Chapter } 1 & \text { General introduction } & 7\end{array}$

Chapter 2 Uncertainty and coverage with evidence development: 21

Does practice meet theory?

Chapter 3 Fluocinolone acetonide intravitreal implant for treating recurrent non-infectious uveitis: An evidence review group perspective of a NICE single technology appraisal

Chapter 4 Reviewing the quality, health benefit, and value for money of chemotherapy and targeted therapy for metastatic breast cancer

Chapter 5 The relative effectiveness of eribulin for advanced breast cancer treatment: A study of the Southeast Netherlands advanced breast cancer registry

Chapter 6 An economic evaluation of eribulin for advanced breast cancer treatment based on the Southeast Netherlands advanced breast cancer registry

Chapter 7 Increasing the transparency and accessibility of survival analysis for decision analytic models: the PERSUADE template

Chapter 8 Assessing survival model selection uncertainty in cost-effectiveness analyses

Chapter 9 Development and validation of the TRansparent Uncertainty ASsessmenT (TRUST) tool for assessing uncertainties in health economic decision models

Chapter 10 General discussion

Addenda Summary 255

Valorisation

List of publications and presentations

Dankwoord

Author information 



\section{Chapter 1}

General introduction 

In 2018 in the Netherlands, approximately $€ 5.8$ billion was spent on extramural pharmaceuticals ${ }^{1,2}$ and an extra $€ 2.2$ billion was spent on expensive pharmaceuticals administered during inpatient care. ${ }^{3}$ These amounts are expected to rise in the near future due to, among other things, the increasing use of targeted therapies, and the escalating launch prices of (oncology) medicines. ${ }^{4}$ Without adequate regulation, the widespread use of these pharmaceuticals in clinical practice may threaten the affordability of our health care system.

Certainly, these pharmaceuticals aim at improving patients' health, but they come at a price. Patients desire rapid access to new promising pharmaceuticals, physicians wish to deliver effective and safe care to their patients; while, at the same time, decision makers want to guarantee timely access to (new) pharmaceuticals within the limited available budget. To ensure the available healthcare budget is optimally used, only pharmaceuticals providing value for money should be reimbursed. Value for money of a new pharmaceutical is assessed by comparing its costs and benefits with the costs and benefits of the next best care option. Indeed, administering a new pharmaceutical will lead to health gains and costs, but it implies the health gains delivered by administering the next best care option will not be experienced. These non-experienced health gains, or health losses, are also called opportunity costs. ${ }^{5}$ This notion of opportunity costs is crucial in the optimal allocation of the health care budget.

At the time of reimbursement decisions, the available evidence to decision makers on the effectiveness, safety, and costs of pharmaceuticals is often immature and incomplete. This may lead to uncertainty concerning their value for money, or cost effectiveness. Ultimately, this uncertainty creates a potential risk for population health because it increases the likelihood of taking a 'wrong' decision based on the available evidence. In this context, a 'wrong' decision means that one decides to reimburse a cost-ineffective pharmaceutical. One way to reduce that uncertainty is to collect and wait for additional information through clinical research.,

The current thesis focuses on how uncertainty information is used during reimbursement decisions of (new) pharmaceuticals and on developing and applying methods to identify and assess that uncertainty.

\section{Health technology assessment}

Health technology assessment (HTA) is a multidisciplinary discipline focussing on assessing the value of health care technologies, which encompasses pharmaceuticals, medical devices, and the organisation of health care process. ${ }^{8}$ The different domains 
of HTA concern the health problem and the current use of health care technologies, the technical characteristics of health care technologies, the safety, clinical effectiveness, and cost (effectiveness) of health care technologies, as well as ethical, legal, organisational, and patient and social aspects associated with (not) reimbursing these health care technologies (HTA Core ModelC) $)^{9-11}$ Health economic evaluation is a branch of HTA focussing on assessing the value for money of health care technologies. The current thesis focuses on health economic evaluation of pharmaceuticals. In health economic evaluations, the costs and benefits associated with a 'new' pharmaceutical (the intervention) are compared with the costs and benefits associated with the next best care (the comparator). ${ }^{12}$

In the Netherlands, the costs considered in a health economic evaluation are the costs associated with acquiring and administering the pharmaceuticals under study as well as other costs incurred by patients within the health care system. ${ }^{13}$ Besides health care costs, the economic burden of disease and treatment on patients and their families, such as travel costs to the hospital, are taken into account, as well as the costs in other sectors. ${ }^{14,15}$ Examples of costs in other sectors are the costs due to lost productivity ${ }^{16}$ or the costs of specialised education. The effects of pharmaceuticals are valued in terms of their impact on mortality and morbidity. These effects are usually combined in a quality-adjusted life year (QALY) estimation. QALYS are obtained by multiplying longevity of life by health-related quality of life. The QALY is the principal measure of effects in health economic evaluations informing reimbursement decisions because it can be measured through generic instruments across a variety of disease, which ensures the comparability of results of health economic evaluations across disease areas. ${ }^{17}$

The results of health economic evaluations are summarised in an incremental cost-effectiveness ratio (ICER). The ICER is calculated by dividing the difference in costs incurred by using the intervention and its comparator by the difference in effects (or QALY) obtained with these two treatment options. ${ }^{12,18}$ In practice, the new intervention often results in higher health benefits and costs. In those cases, the intervention is assumed to deliver value for money when the ICER is below a predefined willingness-to-pay threshold (the amount of money the population is willing to pay for an additional QALY). ${ }^{19}$ Several jurisdictions use an explicit willingness-to-pay threshold to determine value for money. In the Netherlands, the willingness-to-pay threshold is dependent on disease severity and lies between $€ 20,000$ and $€ 80,000$ per QALY gained. ${ }^{20}$ In the United Kingdom (UK), the willingness-to-pay lies between $£ 20,000$ and $£ 30,000$ per QALY gained. Besides value for money, three other criteria are considered during reimbursement decisions in the Netherlands: 'necessity' (Is a claim for solidarity warranted based on the severity of the disease and the expenses 
associated with obtaining access to treatment?), 'clinical effectiveness' (Does the treatment work? Is it at least as effective as current care?), and 'affordability' (Is it feasible to reimburse the treatment through the basic insurance package?). ${ }^{21}$

Health economic evaluations informing reimbursement decisions are usually performed using mathematical models. ${ }^{18}$ These model-based health economic evaluations play a prominent role in reimbursement decisions because they facilitate data synthesis and extrapolation. Firstly, data synthesis is necessary because a single source of evidence does not provide all the evidence required for performing a health economic evaluation. ${ }^{22,23}$ For instance, a randomised controlled trial (RCT) does not measure all relevant costs and effects of treatments, and may not compare the intervention against all relevant comparators for a specific decision context. ${ }^{22-24}$ Besides, RCTs are limited in time, and patients enrolled in RCTs may not be representative of patients encountered in clinical practice. ${ }^{25}$ To overcome these shortcomings of RCTs, real-world data is routinely used as an additional source of evidence informing health economic evaluations. ${ }^{26}$ Real-world data may be obtained through patient registries, national statistics, claims databases, or other observational research. The potential advantages of real-world data are that the included patients are more representative of patients encountered in clinical practice, the larger sample size, the variety of treatments administered to patients, the longer follow-up of these studies, and the possibility to observe (adverse) events that may not have occurred during RCTs. ${ }^{25}$ The main disadvantage of real-world data is its non-randomised character, which complicates its use for the estimation of the relative effectiveness of the intervention versus its comparators. Due to these shortcomings of both RCT and real-world data, their synthesis through health economic models is inevitable to provide relevant information to decision makers. ${ }^{23}$ Secondly, the available evidence at the time of reimbursement decision may be immature, which causes the need to extrapolate the available evidence to obtain estimates of total costs and effects over a relevant time horizon (often life time). Extrapolation of time-to-event data is frequently performed through parametric survival modelling. ${ }^{27,28}$ In addition, it is common practice to extrapolate evidence from a certain context to the decision context of interest because collecting all relevant evidence in each individual decision context is practically unfeasible. For instance, utility values may be obtained from a population affected by a slightly different disease or originating from another geographic area.

The reimbursement decision process for pharmaceuticals often contains two stages: the assessment and the appraisal phase. During the assessment phase, the pharmaceutical company gathers and analyses all available evidence concerning the clinical and cost effectiveness of a pharmaceutical in the company submission. This 
company submission is then critiqued by HTA agencies. In the Netherlands, the company submission is critiqued by the Dutch Health Care Institute (Zorginstituut Nederland, ZIN), which considers all four basic insurance package criteria (necessity, clinical effectiveness, cost effectiveness, and affordability). In the UK, the clinical and cost-effectiveness evidence of pharmaceuticals is critiqued by an independent academic group, called the evidence review group. After this assessment phase, the company submission and critique, as well as comments from different stakeholders on this evidence, are appraised. During this phase, the decision whether to reimburse a new pharmaceutical is taken by the Ministry of Health (the Netherlands), or by an Appraisal Committee of NICE (UK). The broader societal impact of reimbursing the pharmaceutical, such as equity concerns, are taken into account during this later phase. In the UK, this process (assessment and appraisal) is called Single Technology Appraisal. $^{29,30}$

\section{Uncertainty in health technology assessment}

Health economic models enable to assess the uncertainty affecting the decision at hand. Uncertainty arises because models are simplified representations of reality and because they are dependent on the features of the available evidence. Hence, there may be a probability that a 'wrong' decision is taken due to this uncertainty, which leads to health losses. This probability may be estimated by parameterising the uncertainty surrounding the different (uncertain) elements incorporated in the health economic models.

Parameterising uncertainty helps to determine whether further (and which type of) research concerning the costs and benefits of pharmaceuticals is worthwhile through value of information (VOI) analysis. ${ }^{6,731,32}$ To determine the potential value of further research, one needs to know (a) the probability that the pharmaceutical is cost effective, and (b) the consequences associated with reimbursing a cost-ineffective pharmaceutical. ${ }^{6}$ These two metrics can be combined in the expected value of perfect information (EVPI). The EVPI is often interpreted as the maximum expected benefits that can be obtained from additional research by eliminating all uncertainty. The EVPI is also a measure of risk since it provides a quantification of the expected health losses incurred by the uncertainty surrounding the decision. ${ }^{33}$

The first step in accurately estimating the EVPI is a systematic identification of all uncertainty. ${ }^{34}$ In model-based health economic evaluations, the following types of uncertainty are usually considered: stochastic uncertainty, heterogeneity, parameter uncertainty, and structural uncertainty. ${ }^{35}$ Stochastic uncertainty refers to the inherent 
variability surrounding the occurrence of events, which means that similar individuals will not experience the same costs and effects when receiving the same treatment. A part of stochastic uncertainty, called heterogeneity, may be explained by patient characteristics, such as gender or age. The influence of heterogeneity on the results of health economic models is sometimes explored through subgroup analyses while the unexplained part of stochastic uncertainty is often not considered in health economic models. Parameter uncertainty arises because parameters are estimated based on empirical evidence. Parameter uncertainty is mainly considered to be a consequence of statistical uncertainty, but other sources of parameter uncertainty exists. For instance, conflicting evidence, lack of generalizability of evidence to the real-world setting, and the absence of evidence may cause parameter uncertainty but these sources are often overlooked. Structural uncertainty results from the assumptions underlying the relations between different elements of the model. Finally, decision uncertainty is the cumulative uncertainty resulting from all these types of uncertainty.

Once identified, the impact of all these uncertainties on the results should be assessed through probabilistic sensitivity analyses (PSA), which is usually required for estimating the EVPI. A PSA starts with assigning a statistical distribution to each element of the model (model inputs, structural assumptions). Assigning these statistical distributions aims at parameterising the uncertainty surrounding the (mean) value of each of these elements. Afterwards, a PSA can be performed through Monte Carlo simulation, during which 1,000 (or more) random iterations are drawn from these statistical distributions. ${ }^{35,36}$ The results of each iteration are then calculated based on each random parameter set and averaged. This implies that a statistical distribution should be assigned to all uncertain elements of the model (and be included in the PSA) for the EVPI to accurately reflect decision uncertainty.

Statistical distributions are easily estimated for input parameters estimated based on empirical evidence. Methods have also been developed to capture structural uncertainty, such as parameterising the structural uncertainty, model averaging, and the discrepancy approach. ${ }^{37-39}$ However, these methods remain in the realm of science and are not commonly used in HTA decision making because (a) capturing structural uncertainty is computationally intensive, (b) it is less straightforward to assign a distribution to structural elements of an assessment, and (c) it may be seen as non-informative for health care decision making. As a result, PSAs mainly capture uncertainty caused by statistical imprecision surrounding parameter estimates ${ }^{40}$, and do not reflect more fundamental uncertainties arising from inadequacy of the data to the decision context, and from the absence of evidence. ${ }^{34}$ Not considering these uncertainties leads to an underestimation of the 'true' decision uncertainty ${ }^{33,41}$, which may lead to suboptimal decision making (Figure 1.1). 


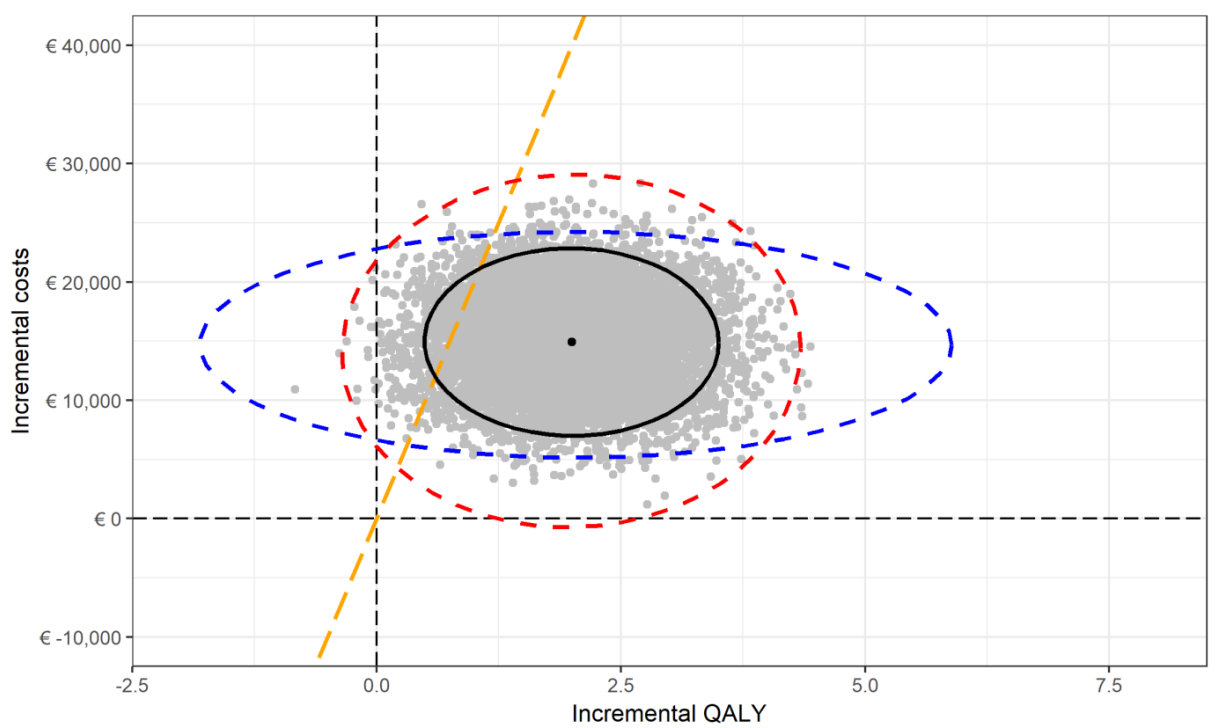

Figure 1.1: Incremental cost-effectiveness plane representing the results of a probabilistic sensitivity analysis.

Each grey dot represent an iteration of the Monte Carlo simulation.

The black dot and its ellipse represent the mean incremental $Q A L Y$ and costs and the uncertainty surrounding these results.

The red and blue ellipses represent the 'true' uncertainty surrounding the results in case several uncertainties have not been included in the probabilistic sensitivity analysis.

The orange line represent the willingness-to-pay threshold (€20,000 per QALY gained).

Abbreviation: $Q A L Y=$ quality-adjusted life year.

\section{Uncertainty in reimbursement decisions}

For an optimal management of uncertainty (and the potential risk it incurs) in reimbursement decisions, transparency concerning uncertainty information is crucial. However, society turns to science to obtain certainty and is not always willing to accept the uncertainty surrounding the decisions to be taken. ${ }^{42}$ This means that overconfidence may be placed in the results of health economic evaluations, because several uncertainties may (purposely) be ignored. Currently, uncertainty information is barely considered during decision making. ${ }^{43}$ Decision makers often rely on a 'most plausible' ICER, which is estimated based on their preferred model assumptions, and risk metrics such as the EVPI are not routinely considered. ${ }^{43}$ Consequently, decision makers are unaware of the potential health losses that will be incurred by a certain decision or whether commissioning further research would be worthwhile. Providing 
this information is key to prevent these potential health losses, especially when considering that policy tools are available to decision makers to manage these uncertainty and risk associated with reimbursement decisions. ${ }^{44}$ These policy tools are commonly gathered under the umbrella term managed entry agreements (MEAs). ${ }^{44-46}$

In the Netherlands, coverage with evidence development (CED) is a type of MEA that has been implemented to regulate the uptake of interventions with proven efficacy but with uncertain cost effectiveness in clinical practice. CED constitutes of temporarily reimbursing an intervention while additional evidence is collected on its (cost-)effectiveness in clinical practice, most often through patient registries. At the end of the CED research period, a definitive reimbursement decision is taken. ${ }^{7,44,45,47-49}$ CED schemes have been implemented in the Netherlands because the collected evidence should reduce the uncertainty surrounding reimbursement decisions. Collecting evidence within clinical practice however has disadvantages. The evidence obtained from registries lacks randomisation and the number of included patients may turn out to be lower than expected. The collected data may thus increase the level of uncertainty or add another uncertainty. ${ }^{50}$ Additionally, the relevance of CED schemes may be undermined because their value is not systematically assessed before the initiation of CED research or because they do not address the most relevant uncertainty. ${ }^{51}$

For a CED scheme to deliver valuable information, it should address the most relevant uncertainty. This emphasises the need of an a priori systematic identification of uncertainty, and an assessment of the risk associated with a decision, but also of the potential value (through VOI analysis) and feasibility of additional research. These steps should be cornerstones of HTA before the implementation of CED, or any other MEA $^{7,24,33,52}$; however, such a priori assessments are still rare. ${ }^{24,53}$

\section{Aim and outline of this thesis}

Health care systems will face many challenges in the future. Drug prices are expected to rise and the evidence base for reimbursement decisions are increasingly uncertain because studies include smaller number of patients, the follow-up of studies are too short to capture relevant outcomes for decision makers and there is an increasing reliance on non-randomised studies to inform reimbursement decisions. ${ }^{54}$

Therefore, the aim of this thesis was to apply and develop methods to identify and assess uncertainty in HTA. More specifically, this thesis focuses on the following three questions: how is uncertainty information currently considered in 
reimbursement decisions? How to assess uncertainty in HTA? And how to improve the uncertainty identification and assessment in HTA?

\section{How is uncertainty information currently considered in reimbursement decisions?}

Chapters $\mathbf{2}$ and $\mathbf{3}$ describe how uncertainty information is currently considered in reimbursement decisions in the Netherlands and the UK. For this purpose, we investigated which types of uncertainty were identified in three Dutch CED cases before CED research initiation and whether these identified uncertainties informed CED research design (Chapter 2). Additionally, stakeholders involved in the different CED cases were interviewed on issues and ways forward with uncertainty in CED schemes. Chapter 3 presents a review of the reimbursement decision taken by NICE concerning fluocinolone acetonide intravitreal implant for the treatment noninfectious uveitis of the posterior segment in the UK. This chapter discusses how uncertainty was assessed and appraised and which uncertainty information influenced the decision the most.

\section{How to assess uncertainty in HTA?}

Chapters 4 to 6 focus on applying methods to consider uncertainty in the assessment of chemotherapy for the treatment of advanced breast cancer ( $A B C)$ patients. Chapter 4 is a literature review of model-based health economic evaluations for $A B C$ treatment. This chapter contains an assessment of how study characteristics related to study quality, and results of the included model-based health economic evaluations. Following, Chapter 5 estimates the relative effectiveness of eribulin (a novel chemotherapy) versus non-eribulin chemotherapy for the treatment of $A B C$ patients. Since registry data informed this study, uncertainty arose due to the absence of randomisation and the potential indication bias. Genetic Matching was applied to address this bias. Chapter 6 builds upon Chapter 5 and determines the costeffectiveness analysis of eribulin versus non-eribulin chemotherapy, based on a health economic model and using the same registry data as in Chapter 5. This chapter assesses the uncertainty associated with the decision to reimburse eribulin within the Dutch context. 


\section{How to improve the uncertainty identification and assessment in HTA?}

Finally, Chapters 7 to 9 present methods and tools aiming at improving the identification and assessment of uncertainty information. Chapter 7 support survival model selection and implementation for health economic models. Chapter $\mathbf{8}$ describes a method to capture structural uncertainty stemming from survival model selection in health economic models. This method aims at improving the assessment of uncertainty in health economic models. Finally, Chapter 9 presents a tool aiming at systematically identifying all uncertainty in health economic decision models informing reimbursement decisions.

This thesis is concluded with a general discussion of the findings (Chapter 10). 


\section{References}

1. OECD. Health at a Glance 2019. 2019.

2. OECD. Pharmaceutical Spending 2018 [25/11/2019]. Available from: https://data.oecd.org/healthres/ pharmaceutical-spending.htm

3. Zorginstituut Nederland. Recentste cijfers over uitgaven aan genees- en hulpmiddelen gepubliceerd 2019 [10/12/2019]. Available from: https://www.gipdatabank.nl/actueel/actueel-oktober-2019openbaar

4. OECD. Improving Forecasting of Pharmaceutical Spending - Insights from 23 OECD and EU Countries. OECD; 2019.

5. $\quad$ Palmer S, Raftery J. Economic Notes: opportunity cost. BMJ. 1999;318(7197):1551-1552.

6. Chalkidou K, Lord J, Fischer A, et al. Evidence-based decision making: when should we wait for more information? Health Affairs (Project Hope). 2008;27(6):1642-1653.

7. Claxton K, Palmer S, Longworth L, et al. A Comprehensive Algorithm for Approval of Health Technologies With, Without, or Only in Research: The Key Principles for Informing Coverage Decisions. Value in Health. 2016;19(6):885-891.

8. World Health Organisation. Medical devices $>$ Health technology assessment [18/10/2019]. Available from: https://www.who.int/medical_devices/assessment/en/

9. Lampe K, Mäkelä M, Garrido MV, et al. The HTA core model: a novel method for producing and reporting health technology assessments. International Journal of Technology Assessment in Health Care. 2009;25 Suppl 2:9-20.

10. Liberati A, Sheldon TA, Banta HD. EUR-ASSESS Project Subgroup report on Methodology. Methodological guidance for the conduct of health technology assessment. Int J Technol Assess Health Care. 1997;13(2):186-219.

11. EunetHTA. HTA Core Model [12/03/2020]. Available from: https://eunethta.eu/hta-core-model/

12. Drummond M. F., Sculpher M. J., Claxton K., et al. Methods for the Economic Evaluation of Health Care Programmes. 4th ed. Oxford, New York: Oxford University Press; 2005.

13. van Baal PH, Wong A, Slobbe LC, et al. Standardizing the inclusion of indirect medical costs in economic evaluations. PharmacoEconomics. 2011;29(3):175-87.

14. Drost RMWA, Paulus ATG, Ruwaard D, et al. Inter-sectoral costs and benefits of mental health prevention: towards a new classification scheme. J Ment Health Policy Econ. 2013;16(4):179-186.

15. Zorginstituut Nederland. Richtlijn voor het uitvoeren van economische evaluaties in de gezondheidszorg: Zorginstituut Nederland; 2015. Available from: https://www.zorginstituutnederland.nl/ over-ons/publicaties/publicatie/2016/02/29/richtlijn-voor-het-uitvoeren-van-economischeevaluaties-in-de-gezondheidszorg

16. Krol M, Brouwer W, Rutten F. Productivity costs in economic evaluations: past, present, future. PharmacoEconomics. 2013;31(7):537-549.

17. Weinstein MC, Torrance G, McGuire A. QALYs: The Basics. Value Health. 2009;12:S5-S9.

18. Briggs AH, Sculpher MJ, K. C. Decision Modelling for Health Economic Evaluation. Oxford, New York: Oxford University Press; 2006.

19. Bertram MY, Lauer JA, De Joncheere K, et al. Cost-effectiveness thresholds: pros and cons. Bull World Health Organ. 2016;94:925-930.

20. Zwaap J, Knies S, van der Meijden C, et al. Kosteneffectiviteit in de praktijk. Diemen, The Netherlands: Zorginstituut Nederland; 2015.

21. Zwaap J. Pakketadvies in de praktijk - Wikken en wegen voor een rechtvaardig pakket. Diemen, The Netherlands: Zorginstituut Nederland; 2017.

22. Sculpher MJ, Claxton K, Drummond M, et al. Whither trial-based economic evaluation for health care decision making? Health Economics. 2006;15(7):677-87.

23. Sculpher M. Clinical trials provide essential evidence, but rarely offer a vehicle for cost-effectiveness analysis. Value Health. 2015;18(2):141-2. 
24. Grimm SE, Fayter D, Ramaekers BLT, et al. Pembrolizumab for Treating Relapsed or Refractory Classical Hodgkin Lymphoma: An Evidence Review Group Perspective of a NICE Single Technology Appraisal. PharmacoEconomics. 2019;37(10):1195-1207.

25. Fleurence RL NH, Jansen JP. The Critical Role Of Observational Evidence In Comparative Effectiveness Research. Health Affairs. 2010;29(10):1826-1833.

26. Makady A, van Veelen A, Jonsson P, et al. Using Real-World Data in Health Technology Assessment (HTA) Practice: A Comparative Study of Five HTA Agencies. PharmacoEconomics. 2018;36(3):359-368.

27. Ishak KJ, Kreif N, Benedict A, et al. Overview of parametric survival analysis for health-economic applications. PharmacoEconomics. 2013;31(8):663-675.

28. Latimer NR. Survival analysis for economic evaluations alongside clinical trials--extrapolation with patient-level data: inconsistencies, limitations, and a practical guide. Med decis making. 2013;33(6):743-54.

29. National Institure for Health and Care Excellence. Guide to the methods of technology appraisal 2013. National Institure for Health and Care Excellence; 2013.

30. National Institure for Health and Care Excellence. Guide to the processes of technology appraisal (2018 update). National Institure for Health and Care Excellence; 2018.

31. Wilson ECF. A Practical Guide to Value of Information Analysis [journal article]. PharmacoEconomics. 2015;33(2):105-121.

32. Eckermann S, Willan AR. Expected value of information and decision making in HTA. Health Economics. 2007;16(2):195-209.

33. Grimm SE, Strong M, Brennan A, et al. The HTA Risk Analysis Chart: Visualising the Need for and Potential Value of Managed Entry Agreements in Health Technology Assessment. PharmacoEconomics. 2017;35(12):1287-1296.

34. Grutters JP, van Asselt MB, Chalkidou K, et al. Healthy decisions: towards uncertainty tolerance in healthcare policy. PharmacoEconomics. 2015;33(1):1-4.

35. Briggs $\mathrm{AH}$, Weinstein $\mathrm{MC}$, Fenwick EA, et al. Model parameter estimation and uncertainty: a report of the ISPOR-SMDM Modeling Good Research Practices Task Force--6. Value Health. 2012;15(6):835-42.

36. Claxton K. Exploring uncertainty in cost-effectiveness analysis. PharmacoEconomics. 2008;26(9): 781-98.

37. Afzali $\mathrm{HH}$, Karnon J. Exploring structural uncertainty in model-based economic evaluations. PharmacoEconomics. 2015;33(5):435-43.

38. Bojke L, Claxton K, Sculpher M, et al. Characterizing Structural Uncertainty in Decision Analytic Models: A Review and Application of Methods. Value Health. 2009;12(5):739-749.

39. Strong M, Oakley JE, Chilcott J. Managing structural uncertainty in health economic decision models: a discrepancy approach. Journal of the Royal Statistical Society: Series C (Applied Statistics). 2012;61(1):25-45.

40. Jackson $\mathrm{CH}$, Bojke L, Thompson SG, et al. A framework for addressing structural uncertainty in decision models. Med Decis Making. 2011;31(4):662-74.

41. Alarid-Escudero F, Enns EA, Kuntz KM, et al. "Time Traveling Is Just Too Dangerous" but Some Methods Are Worth Revisiting: The Advantages of Expected Loss Curves Over Cost-Effectiveness Acceptability Curves and Frontier. Value Health. 2019;22(5):611-618.

42. van Asselt MBA, Vos E. The Precautionary Principle and the Uncertainty Paradox. J Risk Res. 2006;9(4):313-336.

43. Sculpher M, Palmer S. After 20 Years of Using Economic Evaluation, Should NICE be Considered a Methods Innovator? PharmacoEconomics. 2020;38(3):247-257.

44. Garrison LP, Jr., Towse A, Briggs A, et al. Performance-based risk-sharing arrangements-good practices for design, implementation, and evaluation: report of the ISPOR good practices for performance-based risk-sharing arrangements task force. Value Health. 2013;16(5):703-719.

45. Klemp M, Fronsdal KB, Facey K. What principles should govern the use of managed entry agreements? International Journal of Technology Assessment in Health Care. 2011;27(1):77-83.

46. Ferrario A, Kanavos P. Dealing with uncertainty and high prices of new medicines: a comparative analysis of the use of managed entry agreements in Belgium, England, the Netherlands and Sweden. Soc Sci Med. 2015;124:39-47. 
47. Foster NL, Mottola K, Hoffman JM. Coverage with evidence development: what to consider. JAMA Neurol. 2014;71(4):399-400.

48. Hutton J, Trueman P, Henshall C. Coverage with evidence development: an examination of conceptual and policy issues. Int J Technol Assess Health Care. 2007;23(4):425-432.

49. McCabe CJ, Stafinski T, Edlin R, et al. Access with evidence development schemes: a framework for description and evaluation. PharmacoEconomics. 2010;28(2):143-52.

50. Brashers DE. Communication and Uncertainty Management. Journal of Communication. 2001;51(3):477-497.

51. Franken MG, Gaultney JG, Blommestein HM, et al. Policymaker, please consider your needs carefully: does outcomes research in relapsed or refractory multiple myeloma reduce policymaker uncertainty regarding value for money of bortezomib? Value Health. 2014;17(2):245-253.

52. Walker S, Sculpher M, Claxton K, et al. Coverage with evidence development, only in research, risk sharing, or patient access scheme? A framework for coverage decisions. Value Health. 2012;15(3):570-579.

53. Dickson R, Boland A, Duarte R, et al. EMA and NICE Appraisal Processes for Cancer Drugs: Current Status and Uncertainties. Appl Health Econ Health Policy. 2018;16(4):429-432.

54. Anderson M, Naci H, Morrison D, et al. A review of NICE appraisals of pharmaceuticals 2000-2016 found variation in establishing comparative clinical effectiveness. J Clin Epidemiol. 2019;105:50-59. 


\section{Chapter 2}

\section{Uncertainty and coverage with evidence development: Does practice meet theory?}

Xavier G.L.V. Pouwels, Janneke P.C. Grutters, Jill Bindels, Bram L.T. Ramaekers, Manuela A. Joore Published in Value in Health 2019;22(7):799-807 


\section{Abstract}

\section{Objectives}

In theory, a successful coverage with evidence development (CED) scheme is one that addresses the most important uncertainties in a given assessment. We investigated 1) which uncertainties were present during the initial assessment of three Dutch CED cases, 2) how these uncertainties were integrated in the initial assessments, 3) whether CED research plans included the identified uncertainties, 4) issues with, and ways forward for managing uncertainty in CED research.

\section{Methods}

Three CED initial assessment dossiers were analysed and sixteen stakeholders were interviewed. Uncertainties were identified in interviews and dossiers and were categorised in different causes: unavailability, indirectness, and imprecision of evidence. Identified uncertainties could be mentioned, described, and explored. Issues and ways forward to address uncertainty in CED schemes were discussed during the interviews.

\section{Results}

Forty-two uncertainties were identified. Thirteen (31\%) were caused by unavailability, seventeen $(40 \%)$ by indirectness and twelve $(29 \%)$ by imprecision. Thirty-four uncertainties (81\%) were only mentioned, nineteen (45\%) were described, and the impact of three $(7 \%)$ uncertainties on the results was explored in the assessment dossiers. Seventeen uncertainties (40\%) were included in the CED research plans. According to stakeholders, research did not address the identified uncertainty while CED research should be designed to focus on these.

\section{Conclusions}

In practice, uncertainties were neither systematically nor completely identified in the analysed CED schemes. A framework would help to systematically identify uncertainty, and this process should involve all stakeholders. Value of information analysis, and the uncertainties which are not included in this analysis, should inform CED research design. 


\section{Introduction}

Reimbursement decisions concerning pharmaceuticals are subject to uncertainty. Over the years, decision makers have employed different policy measures, called managed entry agreements (MEAs), to regulate the reimbursement of new (expensive) health care innovations. According to the different taxonomies of MEAs, their objective often falls within one or more of the following categories: manage the uncertainty surrounding reimbursement decisions, ensure value for money of health care innovation, optimise utilisation of the health care innovation, or limit budget impact. ${ }^{1-4}$ Coverage with evidence development (CED) is one type of MEA, which aims at reducing the uncertainty surrounding the (cost) effectiveness of treatments by granting access to these treatments to patients while additional evidence is being collected.

As mentioned by Garrison et al., assessing the success of CED schemes has to be based on process indicators because quantitatively assessing the value of CED research, as a result of the reduction in decision uncertainty, is difficult. ${ }^{2}$ And indeed, we are not aware of previous evaluations of the value of CED schemes. Tuffaha and Scuffham reviewed three Australian CED schemes and concluded that it was difficult to assess the success of CED implementation in Australia because stakeholders may have different perspectives on the success of these schemes [5]. Stafinski et al. reviewed CED schemes in different jurisdictions (Australia, Canada (the province of Ontario), Italy, the Netherlands and the United States (US)) on the criterion of consistency between the outcomes of the CED research and the coverage decisions, they found that this was the case in 10 out of 17 CED schemes for which the policy outcome was available [6]. In theory, a successful CED scheme is one that addresses the most important uncertainties in the given appraisal. It is therefore crucial that the decision to engage in a CED scheme is informed by 1) a systematic identification of uncertainty, 2) a description of the direction and magnitude of uncertainty, 3) the exploration of the impact of uncertainty on the cost effectiveness results and, ultimately, on decision-making, and 4) the exploration of how uncertainty and its impact on cost effectiveness results can be reduced by collecting additional evidence.

Methods and algorithms justifying the implementation of CED schemes have been proposed in the past and include value of information (VOI) analysis based methods as well as consideration of different issues such as the timing, the reversibility of the decision, the feasibility of the CED research, and ethical issues.7-15 These methods require a clear understanding of the (causes of) uncertainty surrounding the decision. Different taxonomies, such as the one developed by the ISPOR-SMDM taskforce, aid the understanding of uncertainty in general as well as an 
understanding of which uncertainties can possibly be addressed by research. ${ }^{16}$ However, there are differences in language across the uncertainty literature that makes the identification of uncertainty a potentially daunting task. ${ }^{16-20}$ The ISPORSMDM Taskforce discriminates between methodological uncertainty, stochastic uncertainty, heterogeneity, structural uncertainty, and parameter uncertainty ${ }^{16}$, while other frameworks such as the Grading of Recommendations Assessment, Development and Evaluation (GRADE) approach consider evidence to be less certain when it is imprecise or indirect. Inconsistency in the evidence base, study limitations and reporting bias also negatively influence the certainty of evidence according to GRADE. ${ }^{18,21}$

According to a European review, the Netherlands were leading in terms of the number of implemented MEAs. ${ }^{1}$ In the Netherlands, CED has been used to regulate the reimbursement of expensive medicines since 2006 and of specific outpatient pharmaceuticals since 2012. ${ }^{22}$ The Dutch CED process consists of three phases: 1) the initial assessment where the available evidence is summarised and the CED research plan is developed, 2) the CED research during which additional evidence is collected (most often in a register) on treatments use, effectiveness, safety, and resource use, and 3 ) the final assessment, planned after a maximum of four years of conditional reimbursement, where the decision whether to reimburse the pharmaceutical is taken. ${ }^{22,23}$ Previous research has shown that CED in the Netherlands was useful in producing evidence on the use of treatments in daily clinical practice but that it did not always decrease the uncertainty surrounding the cost effectiveness of these treatments. Hence, policymakers should carefully assess whether additional evidence collection will significantly reduce decision uncertainty before engaging in CED. ${ }^{24}$ Currently, structured evaluations of MEAs are lacking ${ }^{2,24,25}$, and their usefulness in maintaining sustainable financing of health care innovations has been questioned. ${ }^{26}$ To the best of our knowledge, no previous study has assessed whether uncertainties were systematically identified before engaging in additional evidence collection, and whether the identified uncertainty informed the focus and design of CED research. As mentioned above, these steps are crucial for determining the value of additional evidence collection.

This study examines whether the practical implementation of CED in the Netherlands met the above-mentioned steps for a successful CED implementation. We investigated 1) which uncertainties were present during the initial assessment of three Dutch CED cases, 2) how these uncertainties were integrated in the initial assessment dossiers, 3) whether CED research plans included the identified uncertainties, and 4) issues with, and ways forward for, identifying and managing 
uncertainties in CED research. We addressed these aims by a combined qualitative and quantitative analysis of three Dutch CED cases.

\section{Methods}

\subsection{Study design and taxonomy of uncertainty}

The first three CED schemes that were completed in the Netherlands were selected. These cases were selected because their initial and final assessment dossiers were published on the Dutch Health Care Institute website ${ }^{27}$ between 2013 and 2014:

- $\quad$ Alglucosidase Alfa (Myozyme ${ }^{\circledR}$ ) for the treatment of Pompe's disease (AA);

- $\quad$ Rituximab (MabThera ${ }^{\circledR}$ ) for the treatment of rheumatoid arthritis (R);

- Trastuzumab (Herceptin ${ }^{\circledR}$ ) for the treatment of humane epidermal growth factor receptor 2 positive early breast cancer $(\mathrm{T})$.

Interviews were conducted with stakeholders involved in the selected CED cases. Since healthy participants have been interviewed, the questions did not have any personal connotations, and that participants were not subjected to any interventions, no ethical approval was required for the current study. Additionally, the initial assessment dossiers of the selected CED cases were analysed. The initial assessment dossiers contain a summary of the clinical and cost effectiveness evidence provided by the company, a summary of the proposed CED research plan and a critique from the assessment committee (i.e. the scientific advisory committee - subcommittee pharmaceuticals) on the submitted evidence and research plan.

We distinguished between three different causes of uncertainty, derived from the ISPOR-SMDM Taskforce and GRADE taxonomies: unavailability, indirectness, and imprecision of evidence. ${ }^{16,18,21}$ The ISPOR-SMDM Taskforce and GRADE taxonomies were used in the current analysis because the former is widely used in the health economics literature and the latter is already used by the Dutch Health Care Institute to rate the quality of the clinical effectiveness evidence submitted by manufacturers. Combining both taxonomies bridges the definition of uncertainty across different stakeholders involved in health technology assessments. Box 2.1 provides the definitions of each cause of uncertainty. The identified uncertainties were assigned to the aspect of the assessment to which they related: effectiveness, safety, quality of life, and resource use. 


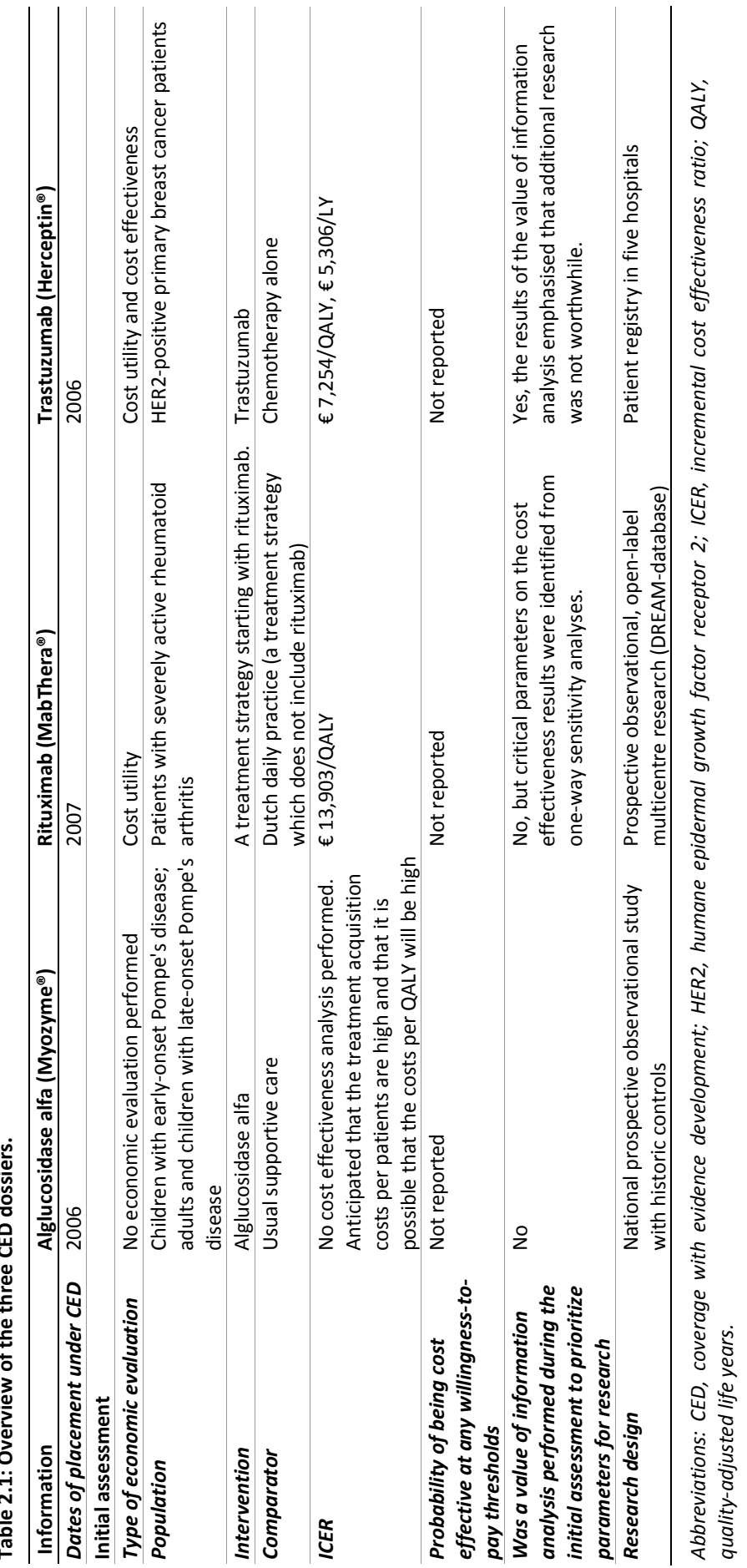


Box 2.1: Definitions of the different causes of uncertainty.

The ISPOR-SMDM Taskforce taxonomy discriminates between methodological uncertainty, stochastic uncertainty, heterogeneity, structural uncertainty, and parameter uncertainty. ${ }^{16}$ In a health economic assessment, heterogeneity and structural uncertainty are, or at least could be, reflected in parameter uncertainty. As such, parameter uncertainty is likely to be the type of uncertainty that is most relevant for CED research, as this type of uncertainty can typically be reduced by conducting empirical research. The Taskforce further specifies that parameter uncertainty can arise because the evidence used in models is based on estimated quantities, the available estimates may not be generalisable to the realworld setting, the available evidence is conflicting, or because evidence is unavailable. ${ }^{16}$ The Grading of Recommendations Assessment, Development, and Evaluation (GRADE) approach to assess the certainty of evidence has been developed. GRADE considers evidence to be uncertain when it is imprecise, indirect, inconsistent, or subject to publication bias. ${ }^{18}$ This taxonomy is very similar to the Taskforce's taxonomy, both considering imprecision, indirectness, and inconsistency as causes of parameter uncertainty. In our view, inconsistency in evidence can be expressed as imprecision when synthesising evidence. In addition, the Taskforce mentions unavailability of evidence and GRADE mentions publication bias; both focusing on evidence that is lacking. Publication bias could also be seen as a source of indirectness when evidence has not been published for the population of interest, for instance. Based on this, we conclude that to identify uncertainty relevant to a CED policy, uncertainty can be categorised as caused by imprecision (a limited number of observations), indirectness (absence of head-to-head comparison between comparators of interest or observations were obtained in a different setting or context) or unavailability of evidence (no observations).

\section{$2.2 \quad$ Interviews}

Face-to-face, semi-structured interviews were conducted in 2014 by JB and either JG or $\mathrm{MJ}$ in the Netherlands. Data collection and analysis of the interviews matched the consolidated criteria for reporting qualitative research (COREQ criteria). ${ }^{28}$ In total, 16 stakeholders [policy makers of ZiNL $(N=5)$, clinicians $(N=5)$, representatives of patient organisations $(\mathrm{N}=2)$, and representatives of pharmaceutical companies $(\mathrm{N}=4)$ ] participated in the semi-structured interviews. Stakeholders were all involved in at least one of the selected CED cases. The stakeholders were approached by e-mail and all approached stakeholders were willing to participate. Stakeholder inclusion continued until data saturation.

The interviews lasted 40-60 minutes. Semi-structured interviews were performed to ensure specific topics were covered during the interviews while encouraging the respondents to talk freely about those topics. ${ }^{29}$ The following subjects were discussed: existing uncertainties during the initial assessments, the management of those uncertainties, and issues with, and ways forward for identifying and managing uncertainties during CED schemes (Appendix 2.1). All interviews were recorded and transcribed verbatim. Content analysis was used to analyse the interview transcripts. ${ }^{30}$ Two researchers (JB and either JG or MJ) independently read the interview transcripts and allocated codes to quotes of the interviews. A report of this first analysis was sent 
to the respondents, asking them to carefully read and comment on it. This technique is called member checking and was applied to validate the analysis. ${ }^{31}$

\subsection{Initial assessment dossiers}

The identification of uncertainty in the initial assessment dossiers was performed in duplicate; XP read all dossiers while JG, BR, and MJ all read one. Identified uncertainties by both assessors were first discussed in couples for each dossier separately. Discrepancies between assessors were discussed within the research team.

Uncertainties were considered as being identified in the initial assessment dossiers when they were mentioned, described, or explored. An uncertainty could be merely mentioned, as in "No data have yet been published on quality of life." The description of uncertainty additionally provided an appreciation of the magnitude and/or direction of uncertainty: "The pooled odds ratio (OR) for malignancy was 3.3 (95\% [confidence interval] $\mathrm{Cl}$ 1.2-9.1) and the one for serious infections [was] 2.0 (95\% $\mathrm{Cl}$ 1.3-3.1) for patients in the anti-TNF [tumour necrosis factor] group compared to the placebo group." The exploration of uncertainty, in addition, assessed the impact of uncertainty on the results through deterministic or probabilistic sensitivity analyses (PSA). We differentiated between mention, description and exploration of uncertainties because these three steps are essential for correctly incorporating uncertainty in a standardised VOI framework. The following assumptions were made: described uncertainties were automatically considered to be mentioned, and explored uncertainties were considered to be both mentioned and described even when these uncertainties were not specifically mentioned or described in the initial assessment dossiers.

The identified uncertainties were categorised in different causes of uncertainty, categories of input, and according to whether they were mentioned, described or explored in a data-matrix. XP retrieved whether the initial assessment dossiers contained PSA and VOI analysis results and whether the identified uncertainties were included in the CED research plan of each initial assessment dossier.

\subsection{Analyses}

The proportion of identified uncertainties was calculated per cause (i.e. unavailability , indirectness, and imprecision). Next, the proportion of uncertainties that were identified by stakeholders but were not included in the initial assessment dossiers was calculated, based on the total number of identified uncertainties. We determined the proportion of uncertainties that were mentioned, described, and explored in the 
initial assessment dossiers based on the total number of identified uncertainties. Uncertainties that were considered as an outcome of CED research were compared to the identified uncertainties to assess whether CED research included all identified uncertainties. Finally, issues with, and ways forward for handling uncertainty in CED mentioned by the different interviewees were compared among the interviews and summarised.

\section{Results}

\subsection{The CED cases}

Table 2.1 provides general information concerning the assessment dossiers. The T initial assessment dossier reported performing a PSA and a VOI analysis, but the dossier did not describe which parameters were included in the PSA. The R initial assessment dossier provided cost effectiveness results without specifying whether a PSA was performed. There was no cost effectiveness analysis performed during the initial assessment of $A A$.

\subsection{Identified uncertainties and integration in CED initial assessment dossiers}

In total, 42 uncertainties were identified from the initial assessment dossiers and the interviews. Thirteen (31\%) of the identified uncertainties were due to unavailability, $17(40 \%)$ were due to indirectness, and 12 (29\%) were due to imprecision (Table 2.2). Thirty-four uncertainties (81\%) were retrieved from the dossiers while the remaining eight (19\%) were only brought up during the interviews. These eight uncertainties were caused by unavailability ( 2 out of 13 uncertainties caused by unavailability), indirectness ( 5 out of 17 uncertainties caused by indirectness) and imprecision (1 out of 12 uncertainties caused by imprecision). Based on the information available in the initial assessment dossiers, 19 (45\%) uncertainties were described and the impact of 3 (7\%) uncertainties on the results was explored through sensitivity analyses (Figure 2.1).

The uncertainties caused by imprecision were relatively more often described (75\%) than the uncertainties due to unavailability (23\%) and indirectness of evidence (41\%). Three uncertainties due to indirectness (18\%) were explored in sensitivity analyses, while this was not the case for any uncertainty due to unavailability or imprecision (Table 2.2). The uncertainties due to indirectness were explored through deterministic sensitivity analyses. The numerical results of these analyses were not provided, but it was stated that these uncertainties were the most influential on the results. 


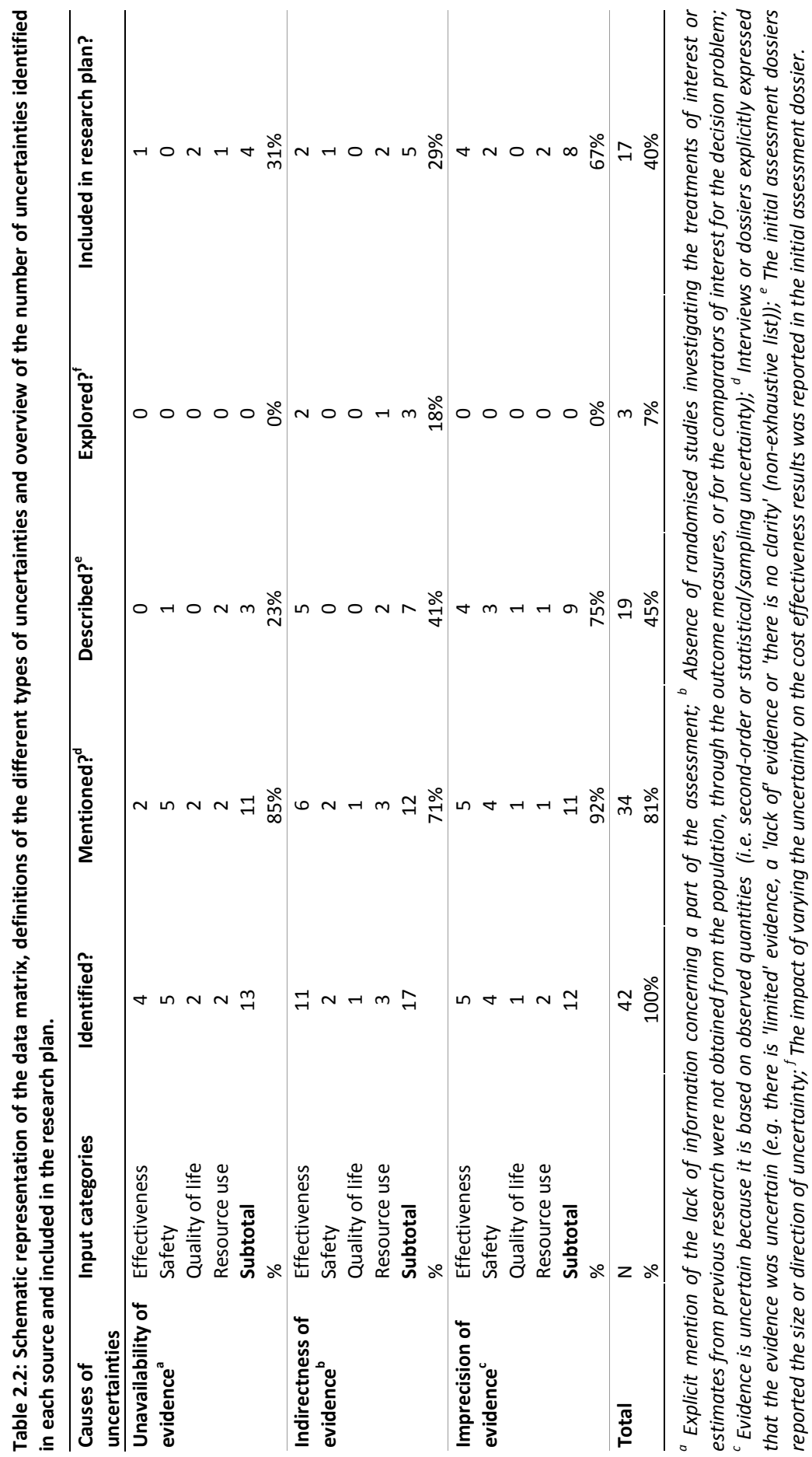




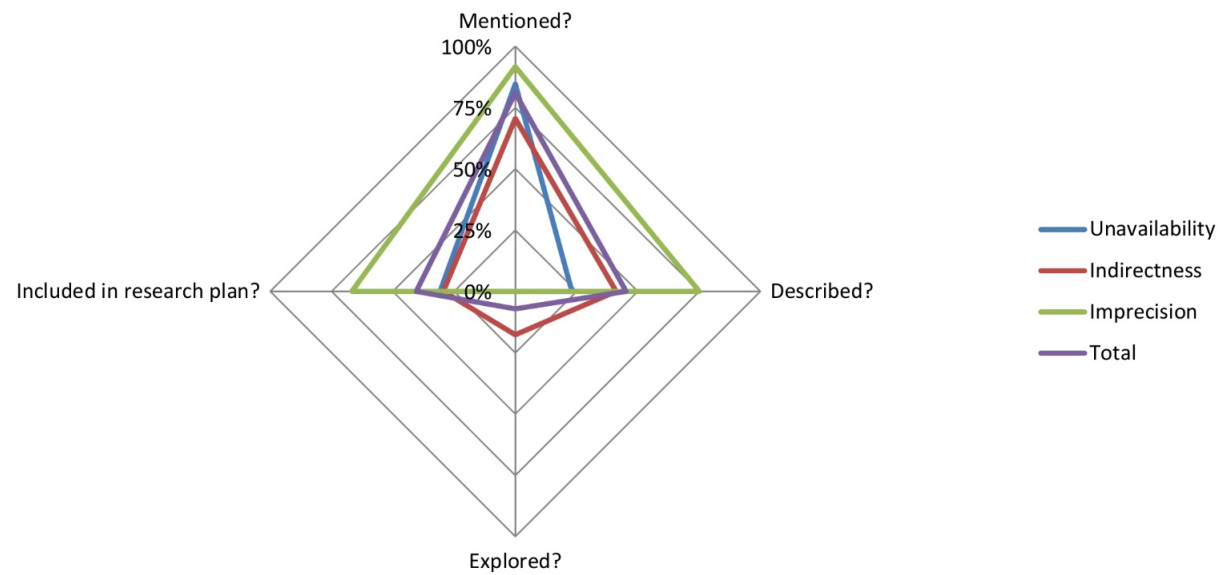

Figure 2.1: Identification of uncertainty and uncertainties included in the CED research plans per cause of uncertainty.

\subsection{Planned research}

In total, 17 out of the 42 uncertainties (40\%) were incorporated in the research plans. Of those 17 uncertainties, 4 were caused by unavailability (31\% out of 13 uncertainties caused by unavailability), 5 by indirectness ( $29 \%$ out of 17 uncertainties caused by indirectness), and 8 by imprecision of evidence ( $67 \%$ out of 12 uncertainties caused by imprecision) (Table 2.2, Figure 2.1). Three uncertainties, which were not identified in the initial assessment dossiers, were included in the research plan; 1 was caused by unavailability ( $8 \%$ out of 13 uncertainties caused by unavailability), 1 by indirectness ( $6 \%$ out of 17 uncertainties caused by indirectness) and 1 by imprecision ( $8 \%$ out of 12 uncertainties caused by indirectness). The VOI analysis performed in $T$ indicated that additional evidence collection was not worthwhile, although not all uncertainties were incorporated.

All three CED research plans aimed at setting up (or continuing data collection through) a disease-specific patient register, i.e. information was collected on all patients eligible to receive the intervention, regardless of the treatment they actually received. In addition, the CED research plans of $A A$ and $T$ aimed at obtaining quality of life estimates in the populations of interest, which were unavailable during the initial assessment. Uncertainties caused by unavailability, indirectness and imprecision relating to the use of treatments in clinical practice were included in the CED research plans. Uncertainties, which were caused by indirectness and imprecision, concerning 
the (relative) effectiveness of treatments and their safety were also included in the CED research plan.

Uncertainties caused by unavailability of evidence that were not included in the CED research plans mainly concerned the (long-term) safety of treatments, the longterm effectiveness of treatments and the modelling of the treatment pathway of patients. Uncertainties caused by indirectness that were not included in the research plan concerned the effectiveness of treatments (e.g. heterogeneity, lack of head-tohead comparison between comparators of interest), the use of treatments in daily clinical practice, and the relevance of the outcome measures. Uncertainties caused by imprecision that were not included in the CED research plans mainly concerned the safety of treatments. The analysed dossiers did not justify why specific outcomes were (not) included in the CED research plan.

\subsection{Issues with, and ways forward for handling uncertainties in CED}

During the interviews, different issues were raised concerning the identification and management of uncertainty during the CED schemes. One interviewee (pharmaceutical industry representative) mentioned that communicating uncertainty information with decision-makers and the general public was difficult because these stakeholders interpret uncertainty as a lack of effectiveness (Box 2.2, quote 8). A higher tolerance toward lower levels of evidence (e.g. expert opinion, $\mathrm{N}=1$ studies) was advocated when assessing treatments for orphan diseases (patient organisation representative, pharmaceutical industry representative (Box 2.2, quote 7)). Multiple interviewees stated that CED research did not focus on reducing uncertainty (pharmaceutical industry representative, decision-maker) but was experienced as a compensation for conditional reimbursement (decision-maker (Box 2.2, quotes 1 \& 2)). It was also emphasised that CED research objectives were not discussed adequately (pharmaceutical industry representative) and that it was unclear which evidence was expected for the final assessment (patient organisation representative, pharmaceutical industry representative (Box 2.2, quote 3)). Multiple interviewees stated that all stakeholders, especially patients, should be involved in deciding on which outcomes additional research should focus on and which outcome measures are relevant (clinician, and patient organisation and pharmaceutical industry representatives (Box 2.2, quote 4)). It was argued that CED research design should be adapted to the uncertainties which were identified in each specific case (patient organisation representative (Box 2.2, quotes $5 \& 6$ )). 
Box 2.2: Quotes from the interviews and initial assessment dossiers.

1. Respondent: "I actually think that the outcome research [CED research] has been set up more as compensation for extra reimbursement. We do spend extra money for those expensive medicines. As compensation thereof, we receive extra information about how it is administered in daily practice. And what the cost effectiveness in daily practice is. And it has thus less to do with uncertainty. I expect it went like this". (decision maker)

2. Respondent: "Well, when thinking about the CED policy, they [the pharmaceutical industry] wanted to be included in it, because then, they would receive more reimbursement for the medicine, that was always the condition to set up a CED research. Thus, yes, you can see these as two separate things. Not like, we want to reduce uncertainty, no, it was a fact, if you want to be included in the CED policy, you have to perform additional research.

3. Respondent: "Well, research proposal were submitted about what was going to be researched and these proposals were approved by ZonMw [The Netherlands Organisation for Health Research and Development] which had received this assignment (performing and CED research), but CVZ [predecessor of ZIN] never said 'this is where we set the bar', this discussion never took place during the initial assessment. And, that was also something that stakeholders were not considering at that moment. We assume we are going to investigate this, we are going to measure all these outcomes and the results is that it is effective or not. And if it comes out that it is effective, then it is enough, but if you have your data here and that you set your bar there, then there is still a mismatch." (pharmaceutical industry representative)

4. Interviewer: "Yes, I can imagine with the example of Pompe [disease], that, in first instance, we say we will look at the walking test. Just as the person you know, she cannot walk but she can work longer and feels fitter. And then one says it doesn't work because of the walking test. But patients feel that it works, but then based on other criteria. Thus, if it had been agreed during the initial assessment, if I am fitter and I need less oxygen, that would be an improvement for me.

Responder: "Yes, and sometimes with such a walking test, for example, it is often said, does it really matter whether you can walk five or twenty meters and you would say, you and I say you know, is this the difference? Just let it go then, no difference. But twenty meters is well [the distance between] my front door and my car, and if I sit in the car, then I can go anywhere. Thus, the five or twenty meters extend my horizon dramatically if I am a patient. If at a certain point I can still fully use my arms, then I can cook by myself at home, so to speak, if someone brings me the groceries, but I can cook by myself. If I cannot use my arms anymore, then home care has to come and cook for me every day. Thus, sometimes you have to look at the kind of numeric parameters, what it means actually, this difference, for this patient and what he/she can in his/her daily living. [...], and that's where you need patients, who can point that out, who can then say 'hey, this a difference and this is not a difference'[...] You cannot picture yourself not being able to type on a computer nowadays, you and I. You would really be handicapped [if you could not]. And this was not considered by any questionnaire, thus at a certain point in time, there are things that become more relevant, of which you think 'hey we should make that one of the priority criteria'. Because it increases dramatically the quality of life of such a patient whether he can use his computer. That is something you don't think about". (patient organisation representative)

5. Respondent: "And I think, we have to go on with this kind of research [CED research], because we really want to know whether medicines have a real additional value, because they are expensive enough to have a look at it. Only I think you have to think carefully about when you implement it and when you don't have to implement it. And it's a bit the same with the medicines for Pompe and Fabry [diseases], I really don't need to perform a cost effectiveness research because you know beforehand that these are not cost effective. Regardless of how you look at it. And who cares whether it costs two hundred thousand or two and a half hundred thousand euros per QALY. In both cases it is extremely expensive, in both cases, you would say if we would put a limit somewhere, we are not going do it, why would you invest in researching these [medicines]. It is a waste of money and time, and energy. So, you just don't have to do it." Interviewer: "So actually the whole CED process?" 
Respondent: "No, for some other medicines, you can maybe look at the cost effectiveness, but you really need to think carefully when you use that criteria and actually customise [the process] per medicine that will be included in CED schemes, like what are we going to do here and what not". (patient organisation representative)

6 Respondent: "Yes, look, in some instances, I can imagine, if there is uncertainty that you want to know more about the daily practice, that I can totally imagine, there was no rationale behind that we are going to collect this data from the daily practice and we will look four years later what it produces, but we are not going to look individually for each case which uncertainty there are and which additional data are needed to address this uncertainty". (pharmaceutical company representative)

7. Respondent: "This is written in the guidelines if you look at the evidence, that for orphan diseases, exceptions can be made concerning the level of evidence, but this was not taken into account during the appraisal". (pharmaceutical industry representative)

8. "Respondent: [...] Thus, if you just read [the assessment dossiers], without knowledge of the cases, then you can conclude that the [treatment] centres themselves are uncertain about the effects. Whilst when you talk with these centres, especially about Pompe, there are no doubts for the physicians that it [the treatment] works well, only if you read the dossiers and you read about the uncertainty here, and yes does it work for all patients. Undoubtedly not, or not in the same way, but if you ask the ultimate question to these centres, to these doctors: 'Ok, if it doesn't work, are there any patients you would stop treating with this treatment?', then, this is a difficult matter. And this is something that is carefully considered. But finally, there is not enough attention to translate the uncertainty they [doctors] see in the data or in the treatment, to there is so much uncertainty that you have to decide not to administer this treatment to this patient. This also says something, because also doctors are not going to treat patients with a medicine if there is so much uncertainty surrounding the treatment or if the risk benefit analysis is so negative. Then you don't do that, and that also indicates that for them, the equilibrium, with this uncertainty still tilts in one direction. One way or another, they see enough advantage in the treatment for this individual patient." (pharmaceutical industry representative).

\section{Discussion}

In total, 42 of uncertainties were identified (13 due to unavailability (31\%), 17 due to indirectness (40\%), and 12 due to imprecision (29\%)). Thirty-four uncertainties (81\%) were retrieved from the dossiers while the remaining eight (19\%) were only brought up during the interviews. This emphasises that uncertainty was not completely identified in the analysed CED cases. Thirty-four (81\%) of the 42 uncertainties were only mentioned, nineteen uncertainties (45\%) were described in more detail and the impact on the results of three uncertainties (7\%) was explored. Seventeen out of the forty-two uncertainties (40\%) were incorporated in the CED research plans. Uncertainties due to imprecision were more often included (67\%) in the research plans, compared to uncertainties due to unavailability (31\%) or indirectness $(29 \%)$. The analysed dossiers did not justify why specific outcomes were (not) included in the CED research plan. Stakeholders emphasised that CED research design should be 
adapted to the identified uncertainties, and questioned whether research had reduced uncertainty.

These findings support the need for a more systematic approach to identify uncertainties in assessments, and to explore the impact of these uncertainties on the results and decision making. Such an approach should rest on clear definition of uncertainty and its causes. The categorisation of uncertainty according to different causes broadens the focus on uncertainty by not only acknowledging uncertainty due to a lack of precision but also uncertainties that are caused by indirectness and unavailability. In addition, this categorization may assist decisions on CED research design. For instance, unavailability may emphasise that the lacking evidence is difficult to obtain, or that it is not possible to gather the missing evidence in a limited time frame (e.g. long-term outcomes for a rare disease). Indirectness indicates that evidence should be gathered in the context of interest while imprecision suggests that more observations are needed. Ultimately, discussing and communicating uncertainty information openly could increase the tolerance and sensitivity of stakeholders towards uncertainty information. ${ }^{32}$ Identifying uncertainty should be the first step before considering a CED scheme and before assessing the feasibility of the considered research design. ${ }^{8,9,12,13,33}$

The strengths of this study are the use of member checking to validate the analysis of the interviews, and the combination of qualitative evidence obtained from stakeholders involved in the investigated cases with information from the initial assessment dossiers. A limitation of the current study concerns the generalisability of the results because only three cases have been investigated. However, these cases contain examples of the typical caveats to the implementation of CED policies (e.g. lack of systematic uncertainty identification, absence of VOI analysis assessing whether additional evidence collection is worthwhile). Another limitation is that only publicly available documents could be analysed. The classification of uncertainties and the analysis of how uncertainties were integrated in the assessments were thus dependent on publicly available information, which is less comprehensive than the dossiers submitted by manufacturers to the Dutch Health Care Institute. Related to this, the dossiers did not report the importance of the identified uncertainties for decision-making and the reasons for (not) including some uncertainties in the CED research plans. As a result, it is likely that the percentage of explored uncertainty is underestimated, and it remains unclear why some identified uncertainties were (not) included in the research plans.

Further research should be conducted to develop tools to systematically identify all uncertainty in health technology assessments. This systematic identification of uncertainty should include an assessment of the impact of all uncertainty on decision- 
making through VOI analysis. ${ }^{15}$ However, in practice, exploring the influence of all uncertainty through VOI analysis is extremely challenging, if not impossible, because the identified uncertainties may be difficult to quantify. ${ }^{20}$ Some uncertainties will not be included in the VOI analysis, which decreases the relevance of the results of the VOI analysis, and may create pseudo-certainty. ${ }^{19}$ It is thus of utmost importance that stakeholders understand which uncertainties are (not) included in the VOI analysis. For instance, the results of the VOI analysis performed in the T dossier (indicating that additional evidence collection was not worthwhile) were ignored because the economic model rested on assumptions which were not thoroughly explained, as mentioned by the assessment committee. ${ }^{34}$ Involving stakeholders earlier in the process of identifying uncertainty will likely increase their understanding and acceptance of VOI analysis. Stakeholder involvement would also facilitate the incorporation of $\mathrm{VOI}$ analysis in a formalised decision-making process, as shown by Bennette et al.. ${ }^{35}$ Finally, investing in additional evidence collection is a political decision. Taking this into account, Koffijberg et al. developed methods to incorporate decision-maker constraints in VOI analysis. ${ }^{36}$ Further methods should be developed to make VOI analysis more useful and acceptable to decision-makers.

Since our study included Dutch CED cases only, comparing the current results to other jurisdictions is not straightforward due to contextual differences (e.g. different cultures, and reimbursement policies). Previous research however provides information on the experiences with CED in other jurisdictions. These studies formulated similar recommendations in order to improve CED schemes implementation. For instance, based on a review of CED schemes performed in Australia, Canada (the province of Ontario), Italy, the Netherlands and the US, Stafinski et al. emphasise that transparency is crucial when implementing CED schemes. ${ }^{6}$ Based on a review of Australian CED cases, Tuffaha and Scuffham underline the importance of assessing the feasibility of CED research and argue for the involvement of all stakeholders when determining CED research objectives. ${ }^{5}$ They further advocate for a clear pre-specification of the conditions of the CED schemes, something that is lacking in the US context according to Garrison et al.. ${ }^{2}$ To our knowledge, in these jurisdictions, as in the Netherlands, no systematic identification of uncertainties takes place. Therefore, the categorisation of uncertainties presented in this paper is relevant for reimbursement agencies internationally since they increasingly face assessments in which uncertainty is difficult to quantify (e.g. use of single arm trials and/or simulated treatment comparisons ${ }^{37}$ ). The current categorisation helps to express why the presented evidence is uncertain, hopefully helps to raise awareness and acceptance of uncertain information, and assists choices concerning CED research designs. 
In conclusion, uncertainty was not systematically identified nor fully explored in the analysed CED cases, and neither was there a clear link to identified uncertainties and the CED research. A transparent identification of all uncertainty and assessment of the impact of this uncertainty on the results of the assessment is crucial for a successful CED scheme implementation. For this, the development of a practical tool that clearly defines uncertainty, its causes and its impact on assessment results is needed. 


\section{References}

1. Ferrario A, Kanavos P. Dealing with uncertainty and high prices of new medicines: a comparative analysis of the use of managed entry agreements in Belgium, England, the Netherlands and Sweden. Soc Sci Med. 2015;124:39-47.

2. Garrison LP, Jr., Towse A, Briggs A, et al. Performance-based risk-sharing arrangements-good practices for design, implementation, and evaluation: report of the ISPOR good practices for performance-based risk-sharing arrangements task force. Value Health. 2013;16(5):703-19.

3. Klemp M, Fronsdal KB, Facey K. What principles should govern the use of managed entry agreements? Int J Technol Assess Health Care. 2011;27(1):77-83.

4. Panos K, Alessandra F, Giovanni T, et al. Managing Risk and Uncertainty in Health Technology Introduction: The Role of Managed Entry Agreements. Global Policy. 2017;8(S2):84-92.

5. Tuffaha HW, Scuffham PA. The Australian Managed Entry Scheme: Are We Getting it Right? PharmacoEconomics. 2018;36(5):555-565.

6. Stafinski T, McCabe CJ, Menon D. Funding the unfundable: mechanisms for managing uncertainty in decisions on the introduction of new and innovative technologies into healthcare systems. PharmacoEconomics. 2010;28(2):113-42.

7. Chalkidou K, Lord J, Fischer A, et al. Evidence-based decision making: when should we wait for more information? Health Aff (Millwood). 2008;27(6):1642-53.

8. Claxton K, Palmer S, Longworth L, et al. Informing a decision framework for when NICE should recommend the use of health technologies only in the context of an appropriately designed programme of evidence development. Health Technol Assess. 2012;16(46):1-323.

9. Drummond M. When do performance-based risk-sharing arrangements make sense? Eur J Health Econ. 2015;16(6):569-571.

10. Grimm SE, Strong M, Brennan A, et al. The HTA Risk Analysis Chart: Visualising the Need for and Potential Value of Managed Entry Agreements in Health Technology Assessment [journal article]. PharmacoEconomics. 2017;35(12):1287-1296.

11. McKenna C, Soares M, Claxton K, et al. Unifying Research and Reimbursement Decisions: Case Studies Demonstrating the Sequence of Assessment and Judgments Required. Value Health. 2015;18(6): 865-75.

12. Rothery C, Claxton K, Palmer S, et al. Characterising Uncertainty in the Assessment of Medical Devices and Determining Future Research Needs. Health Econ. 2017;26 Suppl 1:109-123.

13. Walker S, Sculpher M, Claxton K, et al. Coverage with evidence development, only in research, risk sharing, or patient access scheme? A framework for coverage decisions. Value Health. 2012;15(3):570-9.

14. Menon D, McCabe CJ, Stafinski T, et al. Principles of design of access with evidence development approaches: a consensus statement from the Banff Summit. PharmacoEconomics. 2010;28(2):109-11.

15. Eckermann S, Willan AR. Expected value of information and decision making in HTA. Health Econ. 2007;16(2):195-209.

16. Briggs $\mathrm{AH}$, Weinstein $\mathrm{MC}$, Fenwick EAL, et al. Model Parameter Estimation and Uncertainty: A Report of the ISPOR-SMDM Modeling Good Research Practices Task Force-6. Value Health. 2012;15(6): 835-842.

17. Walker WE, Harremoës $P$, Rotmans J, et al. Defining uncertainty: a conceptual basis for uncertainty management in model-based decision support. Integrated assessment. 2003;4(1):5-17.

18. Guyatt GH, Oxman AD, Kunz R, et al. What is "quality of evidence" and why is it important to clinicians? BMJ. 2008;336(7651):995-998.

19. Grutters JP, van Asselt MB, Chalkidou K, et al. Healthy decisions: towards uncertainty tolerance in healthcare policy. PharmacoEconomics. 2015;33(1):1-4.

20. Ghabri S, Cleemput I, Josselin J-M. Towards a New Framework for Addressing Structural Uncertainty in Health Technology Assessment Guidelines [journal article]. PharmacoEconomics. 2018;36(2): 127-130.

21. GRADE Working Group. Available from: http://www.gradeworkinggroup.org/ 
22. Zorginstituut Nederland. Beoordeling voorwaardelijk toelating van zorg [06-06-2018]. Available from: https://www.zorginstituutnederland.nl/over-ons/werkwijzen-en-procedures/adviseren-over-enverduidelijken-van-het-basispakket-aan-zorg/beoordeling-voor-voorwaardelijke-toelating-van-zorg

23. Kuijpers MR, WGM T. Procedure beoordeling intramurale geneesmiddelen [Assessment Procedure ofr Inpatient Drugs] (Report No. 26022597). Diemen, The Netherlands: College voor Zoregverzekeringen; 2006.

24. Franken MG, Gaultney JG, Blommestein HM, et al. Policymaker, please consider your needs carefully: does outcomes research in relapsed or refractory multiple myeloma reduce policymaker uncertainty regarding value for money of bortezomib? Value Health. 2014;17(2):245-53.

25. McCabe CJ, Stafinski T, Edlin R, et al. Access with evidence development schemes: a framework for description and evaluation. PharmacoEconomics. 2010;28(2):143-52.

26. Hollis A. Sustainable Financing of Innovative Therapies: A Review of Approaches [journal article]. PharmacoEconomics. 2016;34(10):971-980.

27. Zorginstituut Nederland. [06-06-2018]. Available from: https://www.zorginstituutnederland.nl/

28. Tong A, Sainsbury P, Craig J. Consolidated criteria for reporting qualitative research (COREQ): a 32item checklist for interviews and focus groups. Int J Qual Health Care. 2007;19(6):349-57. d

29. Polit DF, Beck CT. Nursing research: Generating and assessing evidence for nursing practice. Lippincott Williams \& Wilkins; 2008.

30. Hsieh H-F, Shannon SE. Three approaches to qualitative content analysis. Qual Health Res. 2005;15(9):1277-1288.

31. Lincoln YS, Guba EG. But is it rigorous? Trustworthiness and authenticity in naturalistic evaluation. New directions for program evaluation. 1986;1986(30):73-84.

32. van Asselt MBA, Vos E. Wrestling with uncertain risks: EU regulation of GMOs and the uncertainty paradox. J Risk Res. 2008;11(1-2):281-300.

33. Claxton K, Palmer S, Longworth L, et al. A Comprehensive Algorithm for Approval of Health Technologies With, Without, or Only in Research: The Key Principles for Informing Coverage Decisions. Value Health. 2016;19(6):885-891.

34. Zorginstituut Nederland, (Committee on medicinal products). Vraagstelling Doelmatigheidstoets adjuvant gebruikt trastuzumab (Herceptin ${ }^{\circledR}$ ) bij de behandeling van HER2 positieve vroege borstkanker. 2008.

35. Bennette CS, Veenstra DL, Basu A, et al. Development and Evaluation of an Approach to Using Value of Information Analyses for Real-Time Prioritization Decisions Within SWOG, a Large Cancer Clinical Trials Cooperative Group. Med Decis Making. 2016;36(5):641-51.

36. Koffijberg H, Knies S, Janssen MP. The Impact of Decision Makers' Constraints on the Outcome of Value of Information Analysis. Value Health. 2018;21(2):203-209.

37. Grimm SE, Armstrong N, Ramaekers BLT, et al. Nivolumab for Treating Metastatic or Unresectable Urothelial Cancer: An Evidence Review Group Perspective of a NICE Single Technology Appraisal. PharmacoEconomics. 2019;37(5):655-667. 


\section{Appendix 2.1: Interview guide}

\section{Introduction of the subject}

The aim of this project is to investigate how to better address uncertainty during the decision-making process. During this project, we study how uncertainty is currently handled in pharmaceuticals reimbursement decisions. To investigate how uncertainty is currently handled in the decision-making process, we are investigating three cases more thoroughly. Today, we would like to discuss the dossier concerning [name of the drug and indication] and the uncertainty present in this case

- Ask for permission to record

- Explain that summary of the interview will be sent for member check

- Ask for the role of the interviewee in the decision making process concerning the case of interest

2. Uncertainty present during the initial assessment

- Which uncertainties were identified during the initial assessment?

- How were these uncertainties handled?

- What were the key-issues which led to the decision to engage in further research and why?

- Which issues were decisive to engage in further research and why were these decisive elements?

- Which information did you miss and why?

\section{Uncertainty present during the final assessment}

- Which uncertainties were identified during the final assessment?

- How were these uncertainties handled?

- What were the key-issues which influenced the final decision and why?

- Which issues were decisive for the final decision and why?

- Which information did you miss and why?

\section{Reflection on the CED process}

- What could/should have been done differently in this case?

- What should change in the decision-making process or CED policy to handle uncertainty in a more appropriate way? 


\section{Chapter 3}

\section{Fluocinolone acetonide intravitreal implant for treating recurrent non- infectious uveitis: An evidence review group perspective of a NICE single technology appraisal}

Xavier G.L.V. Pouwels, Svenja Petersohn, Vanesa Huertas Carrera, Alastair K. Denniston, Annette Chalker, Heike Raatz, Nigel Armstrong, Dhwani Shah, Willem Witlox, Gill Worthy, Caro Noake, Rob Riemsma, Jos Kleijnen, Manuela A. Joore 


\section{Abstract}

The National Institute for Health and Care Excellence (NICE) invited Alimera Sciences, the company manufacturing fluocinolone acetonide intravitreal implant $0.19 \mathrm{mg}$ (FAc; tradename ILUVIEN ${ }^{\circledR}$ ), to submit evidence on the clinical and cost effectiveness of FAc for treating recurrent non-infectious uveitis. Kleijnen Systematic Reviews Ltd, in collaboration with Maastricht University Medical Centre+, was commissioned to act as the independent Evidence Review Group (ERG). This paper contains a summary of the clinical and cost effectiveness evidence submitted by the company, the ERG's critique on the submitted evidence, and the guidance issued by the NICE appraisal committee (AC). The company submission (CS) was mainly informed by the PSV-FAI-001 trial in which FAc was compared with (limited) current practice $((L) C P)$, which was not considered by the ERG to be representative of UK clinical practice. There was no comparison of FAc to any treatment listed in the final scope and especially to the dexamethasone intravitreal implant (dexamethasone), which was considered to be a relevant comparator by the AC. The primary outcome of the PSV-FAI-001 was recurrence of uveitis in the treated eye. Most of the events for the primary outcomes were imputed during the PSV-FAI-001 trial, which probably led to an overestimation of the number of recurrences of disease, and a biased estimate of the relative effectiveness of FAc versus (L)CP. Finally, the place of FAc in the treatment pathway was not clearly defined by the company. Substantial uncertainty surrounded the cost effectiveness results due to the shortcomings of the clinical evidence. Additionally, the quality of life of patients was not measured during the PSV-FAI-001 trial and long-term effectiveness data of FAc were lacking. The ERG adjusted several issues identified in the company submissions and added dexamethasone as a comparator in the decision analytic model. The ERG presented multiple analyses as base-cases because several elements of the assessments remained uncertain. The fully incremental ERG results ranged from dexamethasone (extendedly) dominating FAc (when assuming a hazard ratio of 1 or 0.7 for dexamethasone versus $F A c$ ) to an incremental cost effectiveness ratio of $£ 30,153$ per QALY gained for FAc versus (L)CP (when assuming a hazard ratio of 0.456 for dexamethasone versus (L)CP). The ICER of FAc versus (L)CP ranged from $£ 12,325$ to $£ 30,153$ per QALY gained. After a second AC meeting where alternative company scenarios comparing FAC to dexamethasone were considered by the $\mathrm{AC}$, the $\mathrm{AC}$ concluded that "the results of the company's analyses ranged from the fluocinolone acetonide implant being dominant (that is, it was more effective and costs less), to an incremental cost-effectiveness ratio (ICER) of $£ 29,461$ per QALY gained, and most of the ICERs were below $£ 20,000$ per QALY gained". Therefore, the $A C$ recommended FAC as a cost-effective use of NHS resources for treating recurrent noninfectious uveitis affecting the posterior segment of the eye in the final TA590 guidance (published July 2019). 


\section{Introduction}

As part of the single technology appraisal (STA) process, the National Institute for Health and Care Excellence (NICE) invited the company Alimera Sciences to submit evidence concerning the clinical and cost effectiveness of fluocinolone acetonide intravitreal implant (FAc) for the treatment of recurrent non-infectious uveitis. NICE is an independent organisation which provides national guidance in the United Kingdom (UK) on promoting good health and preventing and treating ill health in priority areas with significant impact. To be recommended by NICE for use within the UK National Health Services (NHS), health technologies must represent a clinically and cost effective use of the NHS resources. During the STA process, the company submits a company submission (CS) ${ }^{1}$ which is reviewed and criticised by an independent academic organisation, the evidence review group (ERG). This ERG critique is compiled in the ERG report. Both the CS and ERG report are then considered by an appraisal committee (AC) which issues an Appraisal Consultation Document (ACD) indicating the initial guidance concerning the use of the technology. The ACD may be commented upon by stakeholders. These comments may lead to a subsequent ACD or a Final Appraisal Determination (FAD) ${ }^{2}$, which is open to appeal. In this case, there was no appeal. The final TA590 guidance was published in July 2019.

This paper presents a summary of the ERG report, delivered by Kleijnen Systematic Reviews Ltd, in collaboration with Maastricht University Medical Centre $+^{3}$, and the development of the NICE guidance. It highlights key methodological issues emerging from the appraisal.

\section{The decision problem}

The aim of the appraisal, as defined by the NICE final scope, was to assess the clinical and cost effectiveness of FAc within its marketing authorisation for treating recurrent non-infectious uveitis. Uveitis is a sight-threatening inflammation of the components of the eye located in the uvea (iris, ciliary body, and choroid). ${ }^{4}$ It is a significant cause of sight loss in the working age population, and is responsible for up to $15 \%$ of cases with legal blindness. ${ }^{5}$ Sight loss in uveitis is most commonly due to macular oedema (fluid in the central part of the retina), glaucoma (damage to the optic nerve from high intraocular pressure), or cataract. Non-infectious uveitis affecting the anterior part of the eye only is usually easily treated with eye drops and has a low risk of sight loss. In contrast non-infectious uveitis affecting the posterior segment of the eye (NIU-PS) is 
associated with a higher risk of sight loss and cannot be treated by drops alone. NIUPS, which includes intermediate, posterior and panuveitis was the focus of the CS.

There is no consensus clinical guidance concerning the treatment of NIU-PS in the UK. The company considered that FAc could be administered as first-, second- or third-line treatment.

The NICE final scope listed the following comparators ${ }^{6}$ :

- Periocular or intravitreal corticosteroid injections

- Intravitreal corticosteroid implants including dexamethasone intravitreal implant (dexamethasone)

- $\quad$ Systemic corticosteroids

- Systemic immunosuppressive therapies

- TNF-alpha inhibitors including adalimumab

- Best supportive care (when all other treatment options have been tried)

None of these comparators was explicitly compared with FAc in the CS since the company only provided a comparison of FAc versus (limited) current practice ((L)CP), which was the comparator arm of the pivotal trial (PSV-FAI-001).

The CS focussed on a narrower population than defined by the NICE scope but was in line with the marketing authorisation. The place of FAc in the treatment pathway was unclear, which was problematic because the comparators of interest were dependent on the place of FAc in the treatment pathway.

\section{Independent ERG review}

The following sections contain a summary of the evidence submitted by the company and the critique of that evidence by the ERG.

\subsection{Clinical Effectiveness Evidence Submitted by the Company}

The company identified one relevant randomised controlled trial (RCT) of FAc and its comparators in patients with recurrent or persistent NIU-PS. ${ }^{1,7}$ The PSV-FAI-001 trial (NCT01694186) was a 36-month parallel, randomised, double-blind trial, comparing FAc implant $(0.18 \mathrm{mg}$ ) versus (L)CP (sham injection). The study included 129 patients from six countries (USA, India, Israel, UK, Germany and Hungary), with 20 patients from the UK (16 (18.4\%) in the FAc arm and four (9.5\%) in the (L)CP arm). Patients were randomised with a 2:1 ratio to the FAc and $(\mathrm{L}) \mathrm{CP}$ arms of the trial. 
The primary efficacy analysis was performed on the intention-to-treat (ITT) population at six months and compared the proportion of patients in the treatment and control groups who did have a recurrence of uveitis in the study eye in the six months following day 1.

For the primary endpoint ITT analysis, data on recurrence of uveitis was imputed as follows: 1) a patient was considered as having a recurrence when he/she had not previously experienced a recurrence and missed an eye examination for the assessment of recurrence during follow-up; and 2) a patient who had not previously experienced a recurrence and took a prohibited concomitant medication (systemic treatment or local treatment of the study eye) at any time during the study follow-up prior to being considered as having a recurrence.

In terms of recurrence of uveitis in the study eye, results showed statistically significant benefits of FAc over (L)CP at six (27.6\% vs. $90.5 \%)$ and 12 months $(37.9 \%$ vs $97.6 \%)$. There was a clear effect in terms of time to first recurrence of uveitis in favour of FAc when compared to (L)CP. Most recurrences were imputed (23 out of 24 and 26 out of 38 in the FAC and (L)CP arms respectively at 6 months). The results of secondary outcomes showed that more patients in the FAc arm than the (L)CP arm experienced recurrence of uveitis in the fellow eye at 12 months (86.4\% vs. $74.2 \%$ ). In terms of visual acuity in the study eye, results seemed to favour FAc over (L)CP. However, the statistical significance of the results in terms of visual acuity was not initially reported. It was therefore possible that none of these results showed a statistically significant difference. Also, in terms of the need for further corticosteroid treatment, results favoured FAc over (L)CP; but again, the significance of the results was not reported at the time of the submission. Health-related quality of life was not assessed in the PSV-FAI-001 trial.

The incidence of at least 1 treatment emergent adverse event (TEAE) during 12 months of follow-up was $80 \%$ for FAc and $93 \%$ for (L)CP study eyes. Approximately half of the participants in both treatment groups experienced a non-ocular AE during the first 12 months of the study. Sixteen participants, 9 (10.3\%) in the FAc group and $7(16.7 \%)$ in the (L)CP group, experienced protocol-defined serious ocular AEs. The most common serious ocular AEs were increased intraocular pressure (IOP) ( 2 participants [2.3\%]) and cataract (4 participants [4.6\%]) in the FAc group, and macular edema, non-infectious endophthalmitis, and uveitis (each experienced by 2 participants [4.8\%]) in (L)CP study eyes. Serious AEs, ocular or non-ocular, were manageable and did not result in either treatment or study discontinuation. ${ }^{7}$ 


\subsection{Critique of clinical effectiveness evidence and interpretation}

The CS and response to clarification provided sufficient details for the ERG to appraise the searches for eligible studies. Searches were carried out in accordance with the NICE guide to the methods of technology appraisal Sections 5.2.2 and 5.2.4 using a good range of databases. ${ }^{8}$ Additional searches of HTA agencies, clinical trials registries, conference proceedings and reference checking were reported.

No attempt was made to search for most comparators mentioned in the scope (periocular or intravitreal corticosteroid injections, intravitreal corticosteroid implants, systemic corticosteroids, systemic immunosuppressive therapies, and TNF-alpha inhibitors). Only two active comparators were included in the literature search performed by the company: adalimumab and dexamethasone. However, the company decided not to perform an indirect comparison with these two comparators. Therefore, the only comparison presented in the CS was FAc versus (L)CP from the PSV-FAI-001 trial. The company argued that "the (L)CP arm of PSV-FAI-001 is considered largely representative of current practice in the UK for the treatment of uveitic flares and recurrence". ${ }^{1}$ However, the ERG did not agree with this claim. ${ }^{3}$

The PSV-FAI-001 trial did not provide evidence for the use of FAc as first-line treatment. All patients in the trial had received treatment with systemic corticosteroid or other systemic therapies during the 12 months prior to enrolment. Therefore, the ERG believed that the most likely place of FAc in the treatment pathway was in second-line alongside dexamethasone, which made intraocular dexamethasone the most appropriate comparator. A comparison with adalimumab was relevant if the committee believed FAc was a third-line treatment option. Regarding best supportive care, the independent clinical expert consulted by the ERG advised that best supportive care (BSC; i.e. the absence of active treatment) is very rare in active disease.

Results from the PSV-FAI-001 trial showed that FAc provided significant benefits compared to (L)CP in terms of recurrence of uveitis. However, what was reported as recurrence of uveitis was largely prescription of so-called 'prohibited medication', which is not an adequate measure of this outcome. This is because its prescription may be indicated for a number of reasons other than recurrence of uveitis in the study eye, including recurrence in the fellow eye and deterioration of an underlying autoimmune condition. This was likely to have led to an underestimation of treatment effectiveness in each arm of the trial. However, it was not known how this influences the relative effectiveness of FAc versus (L)CP. It is also questionable whether (L)CP was representative of UK clinical practice (particularly since the NICE approval of the 
dexamethasone implant), and the CS did not present any comparisons with another active treatment for NIU-PS (e.g. dexamethasone, corticosteroids or immunosuppressants). Most recurrences in the trial were imputed, which most likely led to a biased estimation of the (relative) effectiveness of FAc. The magnitude and direction of this bias are unknown.

\subsection{Cost effectiveness evidence submitted by the company}

The company conducted three systematic reviews to identify relevant cost effectiveness analyses, health related quality of life studies, and resource use and cost studies in order to inform its assessment. One relevant resource use and cost study was identified during the review process.

A de novo Markov model, including five health states ('on treatment', 'subsequent treatment', 'remission', 'permanent blindness' and 'death'), was developed by the company (Figure 3.1). This model structure was informed by a previous technology appraisal (adalimumab and dexamethasone for treating noninfectious uveitis, TA $460^{\circ}$ ) and only considered the treated eye of patients during the PSV-FAI-001 trial. Patients entered the model in the 'on treatment' health state, and transited to the 'subsequent treatment' health state upon recurrence. It was assumed that patients could only transit to the 'permanent blindness' health state after treatment failure. Patients were considered in 'remission' when they did not experience disease recurrence for a period of two years.

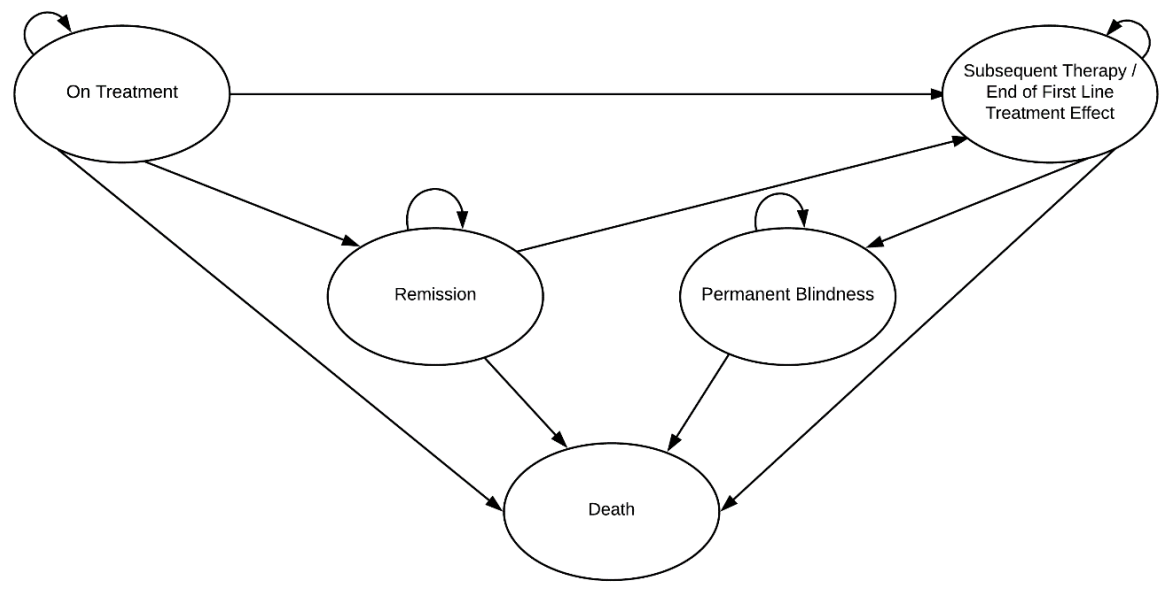

Figure 3.1: Model structure. Source: CS. $^{1}$ 
The cost effectiveness analysis considered FAc versus (L)CP for treating recurrent or persistent NIU-PS, which was is in line with the marketing authorisation. It was assumed that a single FAc implant was implanted at the start of the model. Supplemental treatments (i.e. the treatments that were tapered off at the beginning of the trial) were considered during the first 12 weeks of the model only which meant that patients in the (L)CP arm were assumed not to receive any active treatment for NIU-PS after approximately three months until recurrence.

The model adopted a NHS and Personal and Social Services (PSS) perspective in England and Wales. The cycle length was two weeks and the time horizon was 51 years. A half-cycle correction was not applied. All costs and quality-adjusted life years (QALYS) were discounted at a rate of $3.5 \%$ per year.

The proportion of patients 'on treatment' was informed by the time to first recurrence in the treated eye observed in the PSV-FAI-001 trial and the remaining transition probabilities were informed by the literature. The Kaplan-Meier (KM) curves representing time to first recurrence of the PSV-FAI-001 trial were digitised to inform the proportion of patients 'on treatment' over time. In the FAc arm, a piecewise model was used to model time to first recurrence. The first 120 days of the piecewise model were directly informed by the KM curve and then by a parametric time-toevent model fitted to the remainder of the KM curve. This was justified by the identification of a 'drop' in the KM curve representing time to first recurrence in the FAc arm. In the (L)CP arm, time to first recurrence was modelled through a parametric time-to-event model fitted to the entire KM curve. Multiple distributions (exponential, Weibull, log-logistic, lognormal, gamma, Gompertz, generalised gamma, and generalised F) were fitted to KM curves. The exponential distribution was selected for the FAc arm after 120 days and the log-logistic distribution was selected for the (L)CP arm, based on statistical fit (both distributions had the best statistical fit) and visual inspection. Dick et al. informed the transition from 'subsequent therapy' to 'permanent blindness'. ${ }^{10}$ The transition to 'death' from all health states was equal to UK general population mortality probabilities.

Treatment-related adverse events occurring in at least $5 \%$ of patients in either treatment arm of PSV-FAI-001 were included in the 'on treatment' and 'remission' health states of the cost effectiveness model.

Quality of life data was not measured in the PSV-FAI-001 trial and no relevant studies were identified during the systematic literature review. Hence, the utility values for the 'on treatment' and 'subsequent treatment' health states were obtained through mapping. The company used a mapping algorithm which maps the visual function questionnaire (VFQ)-25 data into EuroQol 5 dimensions (EQ-5D) data. This mapping algorithm was developed during TA460 based on the HURON trial. ${ }^{9}$ In the 
current appraisal, this mapping algorithm was applied to the VFQ-25 data reported in the MUST trial ${ }^{11}$ to obtain the 'on treatment' and 'subsequent treatment' health state utility values. The utility value associated with 'permanent blindness' $(0.38)$ was taken from the literature. ${ }^{12}$ Patients in the 'remission' health sate were assumed to have utility values equal to the age-matched general UK population utility values. ${ }^{13}$ No utility decrements were associated with the occurrence of adverse events.

The following cost categories were included in the cost effectiveness model: acquisition and administration costs of the intervention, monitoring costs, costs of supplemental and subsequent treatments, costs of permanent blindness and costs of managing adverse events. The list price of a single FAc implant was $£ 5,500$ but the company presented results based on a patient access scheme (PAS) price. The 'permanent blindness' costs were divided in one-off transition costs, and cyclic costs. The transition costs contained the costs of registration as a blind person, the costs of low vision aids, low vision rehabilitation, and the costs of residential care, while the cyclic costs contained the monitoring costs, and the costs of depression, hip replacement and community care.

FAc was associated with higher quality-adjusted life year ( $Q A L Y$ ) gains and higher costs than (L)CP. The probabilistic ICER (1,000 iterations) of FAc versus (L)CP was $£ 7,702$ per QALY gained. Based on the deterministic sensitivity analyses (DSA), the most influential parameters on the cost effectiveness results were the 'subsequent treatment' and 'on treatment' health state utility values, and the 45-54 years age matched utility value which informed the 'remission' health state utility value.

\subsection{Critique of cost effectiveness evidence and interpretation}

Literature searches were well documented, reproducible, and performed according to the NICE requirements.

The main shortcomings of the company's model structure were that it did not capture the effect of treatment on patients' visual acuity, and that the company modelled the impact of NIU-PS and treatment in the treated-eye only. The ERG considered this approach inappropriate because patients were at risk of developing bilateral disease. Hence, including the consequences of the disease and treatment in both eyes would have been more appropriate. The use of a 'remission' health state and the lack of transition between the 'on treatment' and 'permanent blindness' health states were not considered to be appropriate by the ERG.

The company's cost effectiveness model considered a single FAc implant. The ERG believed that clinicians may consider to implant subsequent implants in patients who would respond to a first implant. The ERG further wondered about the 
representativeness of (L)CP for UK clinical practice and critiqued the lack of comparison with dexamethasone and other comparators listed in the NICE final scope. Subgroup analyses mentioned in the scope were not performed.

The estimation of time to first recurrence in the cost effectiveness model suffered from the limitations described under Section 3.2. Additionally, the company decided to use a piecewise model to model time to first recurrence in the FAc arm because of the 'drop' identified in the KM curve representing time to first recurrence. However, the company acknowledged that this 'drop' was likely caused by the design of the PSV-FAI-001 trial and not by the influence of treatment on the time to first recurrence; one could wonder whether this 'drop' was representative of UK clinical practice. Uncertainty therefore remains concerning the effectiveness of FAc on time to first recurrence. Besides, the company used different approaches to model time to first recurrence in the FAc and (L)CP arm, respectively a piecewise model and a standard parametric time-to-event model. This may have influenced the outcomes irrespective of differences in treatment effectiveness between FAc and $(L) C P$. The size and magnitude of the bias introduced by all these assumptions could not be quantified by the ERG. Additional uncertainties were introduced by the lack of evidence concerning the effectiveness of FAc after three years, i.e. when the implant is not releasing any active substance anymore, and the use of digitised KM curves instead of individual patient level data.

Quality of life remained uncertain because it was not measured during the PSVFAI-001 trial. Utility values were calculated by applying a mapping algorithm, developed based on data from the HURON trial, to the MUST VFQ-25 data., ${ }^{911}$ The populations in these trials differed from each other and from the population of interest in this appraisal, hence the utility estimates may be biased and their relevance for the current appraisal compromised. Utility decrements for adverse events were not included in the company's analyses and utility values were not capped to UK general population estimates. Additionally, the 'remission' health state utility values were assumed to be equal to UK general population utility value, which was considered to be unrealistic by the ERG. The resulting total QALY estimated by the company in both treatment arms were likely overestimated.

The estimation of the 'permanent blindness' health state costs was not considered appropriate because these were obtained from a macular degeneration population and contained the costs of hip replacement, community care, and residential care. These cost items were not considered to be relevant for the NIU-PS population by the ERG. The ERG further identified multiple errors in the implementation of the cost calculations in the company's cost effectiveness model. 
The probabilistic sensitivity analysis (PSA) did not contain the rate of blindness and almost all parameters were varied by $10 \%$ around the mean. This led to an underestimation of the uncertainty surrounding the results. The ERG further noticed that the scenarios presented by the company did not explore the impact of all structural assumptions on the results.

The ERG was concerned about the quality of the validation efforts performed by the company since multiple implementation errors were identified in the company's cost effectiveness model. Furthermore, the company's experts' opinion elicitation and the validation efforts using data from TA460 were not transparently reported.

\subsection{Additional work undertaken by the ERG}

Based on above described critiques, the ERG amended the company's cost effectiveness model. These amendments involved fixing errors, and including dexamethasone as a comparator (fixing violation). ${ }^{14}$ Since an indirect comparison of FAc versus dexamethasone could not be performed, the ERG presented three sets of scenario analyses assuming a different effectiveness of dexamethasone. In the first set of analyses, the hazard ratio of dexamethasone versus (L)CP was assumed to be 0.456 . This hazard ratio was calculated based on the results of TA460 which compared (L)CP to dexamethasone. To obtain this hazard ratio, the ERG reproduced the incremental QALY gained of 0.029 that was observed in TA460. This estimation rests on the assumption that the incremental QALY gained of dexamethasone versus $(L) C P$ is equal in both appraisals, regardless of the differences in structural assumptions, which was acknowledged to be a strong assumption by the ERG. In the second set of analyses, the same effectiveness was assumed between dexamethasone and FAc (hazard ratio of 1). In the third set of analyses, dexamethasone was assumed to be more effective than FAc (hazard ratio of 0.7 for dexamethasone versus FAc). All hazard ratios were applied to the parametric time-to-event models representing time to first recurrence.

The following additional assumptions were made to incorporate dexamethasone in the cost effectiveness model: dexamethasone was considered to be effective for six months (i.e. a new dexamethasone implant was assumed to be implanted every six months), multiple dexamethasone implants were administered to equal the active treatment period of FAc, the acquisition cost of dexamethasone was $f 870$ and its administration cost was $£ 113.42$. The remaining model inputs and assumptions of dexamethasone were assumed to be the same as for FAc.

The ERG used the individual patient data instead of the digitised KM curve to estimate time to first recurrence, capped utility values to UK general population values, assumed equal supplemental treatment costs between FAc and (L)CP, 
corrected the doses of subsequent and supplemental treatments, and used the standard errors instead of $10 \%$ variation around the mean for the parameters included in the PSA, when available (fixing violations). Multiple matters of judgement were also incorporated in the ERG's preferred analyses: removing the 'remission' health state, including a transition from the 'on treatment' to the 'permanent blindness' health state, assuming the same probability of recurrence after three years in the FAc as in the (L)CP arm, adjusting the 'permanent blindness' health state costs, adding blood tests in the 'subsequent treatment' health state, and assuming the same transition costs to the 'subsequent treatment' health state after three years.

All these amendments were included in the ERG base-case 1 analysis. Three additional ERG base-case analyses were defined. ERG base-case 2 included a utility decrements of 0.05 for all adverse events. ERG base-case 3 assumed that patients could receive multiple FAc implants (the probability of recurrence in the FAc arm after three years was then not assumed equal to probability of recurrence in the (L)CP arm). ERG base-case 4 included both the utility decrements for adverse events and the possibility of receiving multiple FAc implants. Because the model did not allow for a PSA including three treatments, all ERG results were presented deterministically.

FAc extendedly dominated dexamethasone when a hazard of 0.456 was assumed for dexamethasone versus (L)CP. Dexamethasone dominated FAC when assuming equal effectiveness and FAc was extendedly dominated by dexamethasone when assuming a hazard ratio of 0.7 for dexamethasone versus FAc. In all ERG base-case analyses, the ICERs of FAc versus (L)CP remained under $£ 31,000$ per QALY gained (Table 3.1).

Additional exploratory analyses were performed by the ERG. The most influential assumptions, all leading to ICERs above $£ 20,000$ per QALY gained, were using alternative health state utility values for the 'on treatment' and 'subsequent treatment' health state, including higher utility decrements for adverse events (-0.1), and assuming no treatment effectiveness of dexamethasone and FAc after three years.

\subsection{Conclusions of the ERG Report}

The ERG believed that effectiveness and cost effectiveness of FAc were uncertain because (1) of the lack of evidence concerning any relevant comparator, (2) the primary outcome of the trial, 'recurrence of uveitis', was not adequately assessed and the vast majority of recurrences were imputed, (3) of the unavailability of quality of life data elicited in the population of interest. 
Table 3.1: Deterministic company and ERG base-case results.

\begin{tabular}{|c|c|c|c|c|}
\hline $\begin{array}{l}\text { Assumed effectiveness of } \\
\text { DEX }\end{array}$ & Analysis & Comparator & $\begin{array}{l}\text { Fully incremental ICER } \\
\text { (Incremental f/QALY) }\end{array}$ & $\begin{array}{l}\text { ICER of FAC } \\
\text { versus } \\
\text { comparator }\end{array}$ \\
\hline DEX not considered & Company base-case & $(\mathrm{L}) \mathrm{CP}$ & - & $f 7,183$ \\
\hline Hazard ratio of 0.456 for & ERG base-case 1 & (L)CP & Cheapest & $£ 12,325$ \\
\hline \multirow[t]{11}{*}{ DEX versus $(\mathrm{L}) \mathrm{CP}$} & & DEX & Extendedly dominated & $£ 5,335$ \\
\hline & & FAc & $£ 12,325$ & - \\
\hline & ERG base-case 2 & $(\mathrm{~L}) \mathrm{CP}$ & Cheapest & $f 21,531$ \\
\hline & & DEX & Extendedly dominated & $£ 9,457$ \\
\hline & & FAc & $£ 21,531$ & - \\
\hline & ERG base-case 3 & (L)CP & Cheapest & $£ 19,049$ \\
\hline & & DEX & Extendedly dominated & $£ 13,856$ \\
\hline & & FAc & $£ 19,049$ & - \\
\hline & ERG base-case 4 & $(\mathrm{~L}) \mathrm{CP}$ & Cheapest & $£ 30,153$ \\
\hline & & DEX & Extendedly dominated & $£ 22,810$ \\
\hline & & FAc & $£ 30,153$ & - \\
\hline \multirow{12}{*}{$\begin{array}{l}\text { Hazard ratio of } 1 \text { for DEX } \\
\text { versus FAc (i.e. same } \\
\text { effectiveness) }\end{array}$} & ERG base-case 1 & $(\mathrm{~L}) \mathrm{CP}$ & Cheapest & $£ 12,325$ \\
\hline & & DEX & $£ 12,283$ & Dominated \\
\hline & & FAc & Dominated & - \\
\hline & ERG base-case 2 & $(\mathrm{~L}) \mathrm{CP}$ & Cheapest & $£ 21,531$ \\
\hline & & DEX & $£ 21,457$ & Dominated \\
\hline & & FAc & Dominated & - \\
\hline & ERG base-case 3 & $(\mathrm{~L}) \mathrm{CP}$ & Cheapest & $£ 19,049$ \\
\hline & & DEX & $£ 18,710$ & Dominated \\
\hline & & FAC & Dominated & - \\
\hline & ERG base-case 4 & $(\mathrm{~L}) \mathrm{CP}$ & Cheapest & $£ 30,153$ \\
\hline & & DEX & $£ 29,617$ & Dominated \\
\hline & & FAc & Dominated & - \\
\hline Hazard ratio of 0.7 for & ERG base-case 1 & (L)CP & Cheapest & $£ 12,325$ \\
\hline \multirow[t]{11}{*}{ DEX versus FAc } & & FAc & Extendedly dominated & - \\
\hline & & DEX & $£ 10,412$ & $£ 2,297$ \\
\hline & ERG base-case 2 & (L)CP & Cheapest & $£ 21,531$ \\
\hline & & FAc & Extendedly dominated & - \\
\hline & & DEX & $£ 17,843$ & $£ 3,643$ \\
\hline & ERG base-case 3 & $(\mathrm{~L}) \mathrm{CP}$ & Cheapest & $£ 19,049$ \\
\hline & & FAc & Extendedly dominated & - \\
\hline & & DEX & $£ 17,239$ & $£ 12,911$ \\
\hline & ERG base-case 4 & (L)CP & Cheapest & $£ 30,153$ \\
\hline & & FAc & Extendedly dominated & - \\
\hline & & DEX & $£ 25,074$ & $£ 15,730$ \\
\hline
\end{tabular}

Sources: Tables 5.5 and 5.11-5.13 of the ERG report. ${ }^{3}$

Abbreviations: DEX, dexamethasone; ERG, evidence review group; FAc, fluocinolone acetonide; HR, hazard ratio; ICER, incremental cost effectiveness ratio; (L)CP, (limited) clinical practice; $Q A L Y$, quality-adjusted life years. 


\subsection{Company response to first AC meeting and ERG critique}

In response to the first $A C$ meeting, after which a 'minded no' recommendation was issued, the company provided additional cost effectiveness analyses which included some of the ERG's preferred assumptions and considered dexamethasone as a comparator. The ERG and the company analyses differed in how dexamethasone was implemented in the cost effectiveness model. In its response, the company assumed that dexamethasone and FAc were equally effective for their respective active period. The active period of FAc was considered to be three years and the active period of dexamethasone was assumed to be 6 months.

In the company's analyses, the effectiveness of FAc was 'scaled down' from three years to six months to represent the effectiveness of dexamethasone. Hence, the proportion of patients 'on treatment' after six months in the dexamethasone arm was the same as the proportion of patients 'on treatment' after three years in the FAc arm. The company assumed that a subsequent implant was directly administered after treatment failure instead of after the end of the 'active' period of the implants, i.e. 6 months for dexamethasone and 3 years for FAc. The company recalculated the utility decrements associated with adverse events based on additional information about the occurrence of adverse events. The additional analyses of the company contained the following comparisons:

- FAc implant vs. dexamethasone implant (1,2 and 3 implants of each)

- 1 FAc implant vs. 1, 2 and 3 dexamethasone implants

- 1 dexamethasone implant followed by 1 FAc implant vs. 2 and 3 dexamethasone implants

The company response also contained results for bilateral disease. These were obtained by doubling the results from the cost effectiveness model. Finally, a comparison of visual acuity in the FAc and (L)CP arms at different time points was provided, using a one-way ANOVA.

The main critique of the ERG on these additional analyses was that the company did not assume equal effectiveness between FAc and dexamethasone because patients in the dexamethasone arm failed treatment approximately six times faster than patients in the FAc arm. Additionally, the ERG wondered about the relevance of the provided analyses because the time on treatment of 1,2 , or 3 dexamethasone implants is by definition shorter than 1, 2, or 3 FAc implants, hence the FAc arm would automatically lead to higher health benefits. Comparing 1 FAc to 6 dexamethasone implants, as performed in the ERG analyses, seemed more appropriate. Doubling the results of the cost effectiveness model to obtain results for bilateral disease did not capture the effect of treatment on visual acuity in both eyes and consequently on 
quality of life and costs. The reliability of the ANOVA to compare visual acuity results seemed questionable to the ERG because the visual acuity data did not seem to be normally distributed.

\section{Key methodological issues}

It was unclear whether FAc would be administered as a $1^{\text {st }}, 2^{\text {nd }}$ or $3^{\text {rd }}$ treatment line in the treatment pathway. This element of the scope was not clearly defined but was important for the decision to be taken because it determined which comparator was relevant. Additionally, it was unclear whether subsequent FAc implants would be considered. The ambiguity in the scope emphasised that it is of utmost importance to accurately define the scope of an appraisal in order for the company and the ERG to deliver the most informative evidence and analyses.

Another major issue was whether the model structure adequately represented the natural course of the disease since it did not capture the effect of the disease and treatment on visual acuity. The relation between health states, visual acuity, and their associated utility values was thus unclear. Additionally, the company included the consequences of treatment in the treated eye only while NIU-PS is a disease that can develop into bilateral disease. Neglecting the impact of the disease in the second eye of patients therefore biases the estimation of health benefits and costs.

The company's cost effectiveness analyses were highly uncertain because of the multiple shortcomings and uncertainties identified in the evidence submitted by the company (e.g. lack of evidence concerning any relevant comparator, uncertainty surrounding the model structure, and the absence of quality of life data). The impact of these uncertainties on the results was extensively explored by the ERG through scenario analyses and several of these scenario's led to requests for further analyses by the AC. This appraisal therefore shows that scenarios are powerful analyses to support decision-making in case of sparse evidence.

\section{National institute for health and care excellence guidance}

NICE recommended the FAc within its marketing authorisation for treating recurrent non-infectious uveitis on the $20^{\text {th }}$ of June 2019 . This section summarises the committee discussion, full detail can be found in the FAD. ${ }^{2}$ 


\subsection{Intervention}

The AC understood from patient experts that having a long-lasting treatment ( 3 years) would increase patients' quality of life since the treatment of recurrent uveitis requires frequent hospital visits which may disrupt patients' daily life; they also noted that systemic treatments may be relatively contraindicated for some patients. Clinical experts advised that 20 to $30 \%$ of patients do not respond to currently available biologic treatments. For these reasons, a new long-lasting treatment option was deemed valuable.

\subsection{Comparator}

Clinical experts explained that they would consider administering a FAc implant instead of a subsequent dexamethasone implant to patients who originally responded to a dexamethasone implant. Based on this explanation of the potential treatment pathway, the AC deemed that dexamethasone was a relevant comparator for FAc.

\subsection{Considerations of clinical effectiveness}

The AC concluded that the PSV-FAI-001 trial may not represent UK clinical practice because patients in the $(\mathrm{L}) \mathrm{CP}$ arm of the trial did not receive treatment after three months and before recurrence and because investigators were encouraged to first treat recurrence of uveitis with local and then systemic treatment (upon failure of local treatments). This was not considered to represent UK practice because systemic treatment may be administered directly in case of bilateral or systemic disease.

The AC established that the recurrence rate of uveitis was overestimated because recurrences were imputed when patients missed an eye examination, or were treated with local or systemic treatments. The trial did not register why these treatments were administered but the AC noticed that these treatments may have been used to treat the non-study eye or an underlying condition.

The $A C$ recognised that FAC improved visual acuity based on the improvement in best-corrected visual acuity provided by FAc compared to (L)CP. The AC noted that the cost effectiveness model did not capture the effect of treatment on visual acuity.

Cataract and increased intraocular pressure are common adverse events associated with FAc. Clinical experts expected the toxicity profile of FAc to be similar to the toxicity profile of dexamethasone and explained that the adverse events associated with FAc would not lead to a long term decrease in quality of life. The AC concluded that the adverse events profile of FAc was well tolerated and manageable. 


\subsection{Considerations of cost effectiveness}

The model structure of the company only considered the treated eye during the trial. However, clinical experts advised that a large proportion of patients may have bilateral disease (either from outset or becoming bilateral during the course of disease); $67.8 \%$ of patients in the FAc arm and $73.8 \%$ patients in the control arm had bilateral disease at the start of the PSV-FAI-001 trial. Therefore, the AC concluded that a cost effectiveness model considering both eyes would have been more suitable. Additionally, the AC concluded that the cost effectiveness model should not include a 'remission' health state and that both the results with and without transition from the 'on treatment' to 'permanent blindness' health state were relevant.

The AC concluded that multiple implants should be considered in the cost effectiveness model because clinical experts stated that they would consider implanting a subsequent FAc implant to patients who responded to a first implant.

The AC established that dexamethasone was a relevant comparator for FAc. During its deliberations, the AC considered both the ERG and company's methods of implementing dexamethasone in the cost effectiveness model. The AC heard that dexamethasone, over a period of three years (six dexamethasone implants), was expected to be slightly less effective than FAc. The AC concluded that the ERG implementation of dexamethasone was more plausible than the company's implementation of dexamethasone because this later led to an implausible relative effectiveness of dexamethasone and to different treatment durations between the dexamethasone and FAc arms.

Concerning the model inputs, the AC concluded that the cost effectiveness model should not contain treatment benefits of FAc after three years. The AC noted that there was some uncertainty surrounding the methods used to estimate utility decrements associated with AEs in the company's response to the first AC meeting but the AC concluded that the company's method provided more reliable estimates than the ERG method. Finally, the AC stated that the ERG changes concerning the resource use and costs calculated were plausible but had little effects on the cost effectiveness results.

The AC considered FAc to be a cost effective use of NHS resources, had all its preferred assumptions been included in the cost effectiveness model. 


\section{Conclusions}

This paper summarises the STA of FAc for treating recurrent non-infectious uveitis. The ERG identified multiple shortcomings in the evidence submitted by the company such as the lack of evidence concerning any relevant comparator, the likely biased treatment effectiveness estimations, the model structure, and the absence of quality of life data in the population of interest. Even though the $A C$ recognised these gaps in the evidence base, it recommended FAc for the treatment of NIU-PS because the AC considered FAc to be a cost effective use of NHS resource, had all its preferred assumptions been included in the analysis. 


\section{References}

1. Alimera Sciences. Fluocinolone acetonide ocular implant for treating recurrent non-infectious uveitis [ID1039]. Document B: Submission to National Institute of Health and Care Excellence. Single technology appraisal (STA). Alimera Sciences. 2018:1-178.

2. National Institute for Health and Care Excellence. Fluocinolone acetonide intravitreal implant for treating recurrent non-infectious uveitis: Final appraisal document. London: NICE; June 2019.

3. Riemsma R, Pouwels $X$, Petersohn $S$, et al. Fluocinolone acetonide ocular implant for treating recurrent non-infectious uveitis: a Single Technology Assessment. York: Kleijnen Systematic Reviews Ltd; 2019.

4. National Eye Institute. Facts about uveitis [Internet] 2011. Available from: https://nei.nih.gov/health/ uveitis/uveitis. Accessed 29 Nov 2018.

5. Chen SC, Sheu SJ. Recent advances in managing and understanding uveitis. F1000Res. 2017;6:280.

6. National Institute for Health and Care Excellence. Health Technology Appraisal: Fluocinolone acetonide ocular implant for treating recurrent non-infectious uveitis: Final scope. London: NICE. 2018:5.

7. Jaffe GJ, Foster S, Pavesio C, et al. Effect of an injectable fluocinolone acetonide insert on recurrence rates in noninfectious uveitis affecting the posterior segment: 12-month results. Ophthalmology. 2019;126(4):601-610.

8. National Institute for Health and Care Excellence. Guide to the methods of technology appraisal 2013 London: NICE. 2013:93.

9. National Institute for Health and Care Excellence. Adalimumab and dexamethasone for treating noninfectious uveitis. NICE technology appraisal guidance 460 [Internet]. London: NICE. 2017.

10. Dick $A D$, Tundia N, Sorg $R$, et al. Risk of ocular complications in patients with noninfectious intermediate uveitis, posterior uveitis, or panuveitis. Ophthalmology. 2016;123(3):655-662.

11. Multicenter Uveitis Steroid Treatment Trial Follow-up Study Research Group. Quality of life and risks associated with systemic anti-inflammatory therapy versus fluocinolone acetonide intraocular implant for intermediate uveitis, posterior uveitis, or panuveitis: Fifty-four-month results of the multicenter uveitis steroid treatment trial and follow-up study. Ophthalmology. 2015;122(10): 1976-1986.

12. Czoski-Murray C, Carlton J, Brazier J, et al. Valuing condition-specific health states using simulation contact lenses. Value Health. 2009;12(5):793-799.

13. Janssen B, Szende A. Population norms for the EQ-5D. In: Janssen B, Szende A, Cabases J, editors. Selfreported population health: an international perspective based on EQ-5D. Dordrecht: Springer. 2013: 19-30.

14. Kaltenthaler E, Carroll C, Hill-McManus D, et al. The use of exploratory analyses within the National Institute for Health and Care Excellence single technology appraisal process: an evaluation and qualitative analysis. Health Technol Assess. 2016;20(26):1-48. 



\section{Chapter 4}

\section{Reviewing the quality, health benefit, and value for money of chemotherapy and targeted therapy for metastatic breast cancer}




\section{Abstract}

\section{Purpose}

To provide an overview of model characteristics and outcomes of model-based economic evaluations concerning chemotherapy and targeted therapy (TT) for metastatic breast cancer $(\mathrm{MBC})$; to assess the quality of the studies; to analyse the association between model characteristics and study quality and outcomes.

\section{Methods}

PUbMED and NHS EED were systematically searched. Inclusion criteria were: English or Dutch language, model-based economic evaluation, chemotherapy or TT as intervention, population diagnosed with MBC, published between 2000 and 2014, reporting life-years (LY) or qualityadjusted life year (QALY) and an incremental cost-effectiveness ratio (ICER). General characteristics, model characteristics and outcomes of the studies were extracted. Quality of the studies was assessed through a checklist.

\section{Results}

Twenty-four studies were included, considering 50 comparisons (20 concerned chemotherapy and $30 \mathrm{TT}$ ). Seven comparisons were represented in multiple studies. A health state-transition model including the following health states: stable/progression-free disease, progression and death was used in 18 studies. Studies fulfilled on average 14 out of the 26 items of the quality checklist, mostly due to a lack of transparency in reporting. Thirty-one per cent of the incremental net monetary benefit were positive. TT led to higher IQALY gained and industrysponsored studies reported more favourable cost-effectiveness outcomes.

\section{Conclusions}

The development of a disease-specific reference model would improve the transparency and quality of model-based cost-effectiveness assessments for MBC treatments. Incremental health benefits increased over time, but were outweighed by the increased treatment costs. Consequently, increased health benefits led to lower value for money. 


\section{Introduction}

Worldwide, breast cancer is the most incident and prevalent cancer among women (data from 2012). ${ }^{1}$ Due to the incurable character of metastatic breast cancer (MBC) and the intensive health care resources use associated with its management, MBC treatment incurs a high burden on health care budgets. ${ }^{2}$ Policy makers therefore resort to economic evaluations to take coverage decisions concerning $\mathrm{MBC}$ treatments. ${ }^{3}$ These economic evaluations are often based on decision-analytic models (or cost-effectiveness models) because different sources of evidence need to be synthesised and extrapolation of trial results is required to estimate the (lifetime) costs and the impact on survival and quality of life of MBC treatments. Health benefits obtained from $\mathrm{MBC}$ treatments are then weighted against their costs, which provides a measure of value for money of MBC treatments.

Throughout the years, cost-effectiveness models have increasingly been used to support reimbursement decision for new (MBC) treatments and guidelines on good modeling practices have been developed. ${ }^{4,5}$ However, differences in model structure and assumptions, which might influence the cost-effectiveness outcomes ${ }^{6}$, still exist between cost-effectiveness models for MBC treatments. ${ }^{7-10}$ Study sponsorship and quality have also been reported to influence the results of cost-effectiveness assessments. Industry-sponsorship was associated with more beneficial costeffectiveness outcomes for the treatments of interest while higher study quality was associated with less favourable cost-effectiveness outcomes. ${ }^{11}$ Previous research also found that the quality of the cost-effectiveness assessments concerning oncology treatments has not increased over time. ${ }^{12}$ More specifically, a previous review concerning cost-effectiveness models for MBC treatments highlighted the need for high quality studies. ${ }^{13}$

Because model design influences cost-effectiveness results, researchers and the European network for health technology assessment (Eunethta) have argued for more consistency between cost-effectiveness assessments. ${ }^{14-17}$ Eunethta consequently encourages adherence to the HTA Core model ${ }^{\circledR}{ }^{18}$ and researchers have argued for the development of disease-specific reference models; a unique model which would be used for all economic evaluations in a specific disease area. ${ }^{19,20}$

A previous review of cost-effectiveness assessments evaluating chemotherapy and TT for MBC treatment has focussed on identifying the most influential characteristics of the included economic evaluations on the cost-effectiveness outcomes. ${ }^{13}$ However, this previous review did not only include model-based economic evaluations, did not provide an overview of model characteristics, did not assess the quality of the included studies through a standardised checklist and did not 
attempt to illustrate the influence of different model characteristics on study quality and outcomes. The current study consequently aims at 1 ) providing an overview of model characteristics and outcomes of model-based economic evaluations of chemotherapy and TT for MBC treatment, 2) assessing the quality of the included studies and 3 ) investigating the association between model characteristics and study quality and outcomes.

\section{Methods}

\subsection{Literature search and study selection}

PubMed and the National Health Services Economic Evaluation Database (NHS EED) were searched through September and October 2014 (22-10-2014). Existing reviews $^{13,21-24}$ informed the PubMed search query which followed the PICO methodology (patient, intervention, comparator, outcome) (Online Resource, Appendix 4.1). The NHS EED search query was composed of the following terms: "Breast cancer" OR "Breast neoplasm". Inclusion criteria were:

- The study population includes patients diagnosed with advanced or MBC;

- The study is a model-based economic evaluation;

- Chemotherapy or TT is included as a comparator;

- The study reports an incremental cost-effectiveness ratio (ICER) with life years (LYs) and/or quality-adjusted life years (QALYs) as measure of effect;

- The study has been published in English or Dutch as a journal article between January 2000 and October 2014.

$X P$ performed abstract screening. During full text screening, XP reviewed all studies, while BR and MJ each reviewed half of the studies. Disagreements about inclusion were resolved through discussions among all authors. XP performed reference tracking in order to retrieve potentially relevant studies. Inclusion of studies without abstract was assessed during full text screening.

\subsection{Extraction of general information, model characteristics and outcomes}

XP retrieved general information on authors, country, year of publication, comparators, perspective and sponsorship of each study. Through a standardised extraction sheet, the authors retrieved the model characteristics: type of model (the health state-transition model category was composed of "Markov" state transition models and partitioned survival models), health states, treatment effect modeling 
(constant or time-dependent), time horizon, extrapolation beyond trial time horizon, cycle time, adverse events (AEs) (AEs were considered as included when either costs or the effects on quality of life of AEs were incorporated in the model) and subgroup analyses included in the economic evaluations. This was performed in duplicates, discrepancies were discussed among all authors. XP also registered which treatment lines were under investigation in each study. When the treatment line was not clearly stated in the text, it was labelled as 'unclear/mix' because studies might investigate a treatment which is administrated in different treatment lines.

XP extracted information on model inputs: utilities, utility elicitations methods, the type of AEs included and the population (hormonal and human epidermal growth factor receptor 2 (HER-2)-statuses). The following study outcomes were extracted: total LY, QALY and costs for each comparator, incremental costs and effects (incremental LY (iLY) and/or incremental QALY (iQALY)) and ICERs. Total costs, incremental costs and ICERs

were converted to the year 2013 by using the Consumer Price Index of each country. ${ }^{25-27}$ Costs were adjusted to US\$ 2013 and then to $€ 2013$ by using the Purchase Power Parity. ${ }^{28}$ ICERs were rounded to the nearest thousand (or hundred if smaller than 1000). The Net Monetary Benefit (NMB) of each comparator and the incremental NMB (iNMB) of each comparison at a threshold of $€ 40,000$ were calculated.

\subsection{Quality assessment}

Quality assessment of the studies was performed based on a previously used checklist ${ }^{23}$ which consisted of the CHEC checklist ${ }^{29}$ and additional items suggested by Soto. $^{30}$ These additional items concern the type of model, the description of the model and the source of data used in the model. Quality indicators were scored as: yes/complete details given in text (1); no/no details given (0); not clearly stated within text, references given (N.C.); and not applicable (N.A.). ${ }^{23}$ Two authors assessed each study (XP and BR or MJ). Disagreements were resolved through discussions among all authors. The number of items rated as 'yes/complete details given' were summed up for each study in order to obtain an indication of study quality. The checklist contained 26 items.

\subsection{Association of model characteristics with study quality and outcomes}

Graphic plots were used to investigate the association between study quality and study sponsorship, publication year, iQALY and iNMB. Study quality was represented in percentage of correctly described items ('yes/complete details given in text') from the quality checklist. Furthermore, the association between study outcomes (iNMB 
and $\mathrm{iQALY}$ ) and publication year as well as time horizon were explored. A lifetime time horizon was defined as 20 years, as this approximates lifetime in this condition. Finally, the association between $\mathrm{OQALY}$ and $\mathrm{NNMB}$ was investigated.

\section{Results}

\subsection{Literature search}

The literature search provided 1,167 records. From those, 208 were duplicates, 19 were excluded based on language restrictions, 1 was excluded based on its publication date and the abstracts of 9 studies were not available. This resulted in 930 records eligible for abstract screening; of those, 863 were excluded. Full text screening was performed on 77 articles (67 studies identified through abstract screening, 9 studies without abstract, and 1 potential relevant study identified through reference tracking $^{31}$ ). Twenty-four studies ${ }^{8,9,32-53}$ were included (Figure 4.1).

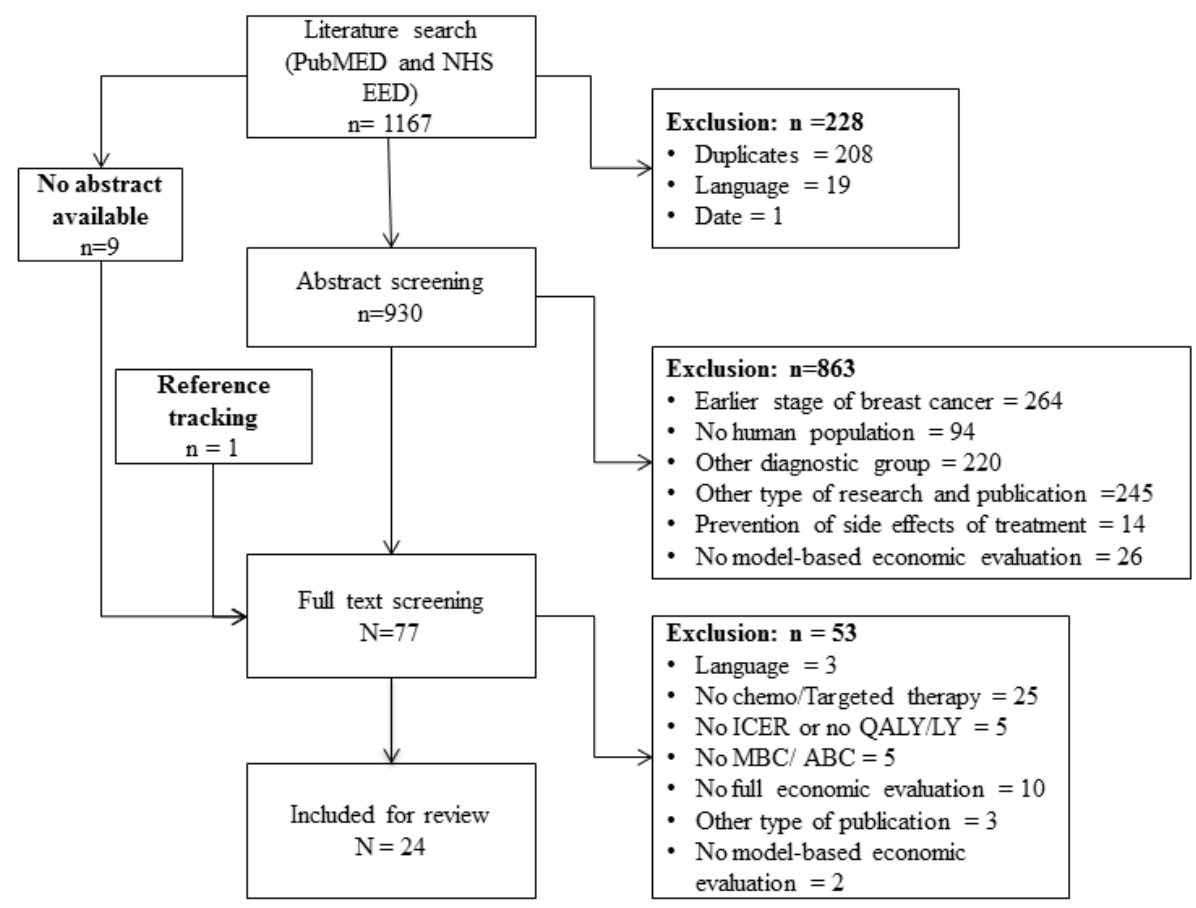

Figure 4.1: Consort diagram of the selection procedure.

Abbreviations: $A B C$, advanced breast cancer; ICER, incremental cost effectiveness ratio; $M B C$, metastatic breast cancer. 


\subsection{General information and models' characteristics of the studies}

Studies were performed in Europe $(\mathrm{N}=14)$, North America $(\mathrm{N}=9)$ and South America $(\mathrm{N}=1)$. Funding by a pharmaceutical company was reported by 11 studies. Two studies used only LY as outcome for the cost-effectiveness assessment, 10 used only QALY and 12 used both LY and QALY. Three studies used a societal perspective, twenty a health care/payer perspective and one used both societal and health care perspectives. The populations in the studies differed with respect to hormonal status, HER-2 status. Studies investigated interventions in different treatment line (Table 4.1). The 24 studies provided 50 comparisons of treatments, 20 concerned chemotherapy and 30 concerned TT. Seven specific treatment comparisons were represented in multiple studies, totaling twenty comparisons, six of them being the same comparisons presented from two different perspectives (health care and societal). The remaining comparisons were only reported in one of the included studies.

Most studies used a health state-transition model $(\mathrm{N}=18)$. The remaining studies used a decision-tree $(\mathrm{N}=2)$, a combination of decision-tree and health state-transition model $(\mathrm{N}=1)$ or did not clearly report which type of model was used $(\mathrm{N}=3)$. Most (18 out of 19) studies using a health state-transition model (either combined with a decision-tree or not), included at least the following three health states: stable/progression-free disease, progression and death. Six of these studies also incorporated a response health state. All studies included AEs, but the number and types of AE differed (Online Resource, Appendix 4.2). Two studies stated they included AEs but did not provide details on which (and how) AEs were incorporated in

the model. ${ }^{51,53}$ Nine studies used a lifetime time horizon, nine studies used a fixed time horizon (varying between 1 and 12 years) and six studies did not clearly define or report their time horizon. Cycle time varied between one day to one and a half month (Table 4.1).

Extrapolation of trial data was described in nine studies. Six studies extrapolated survival data through a parametric survival model assuming a Weibull distribution, two assumed a gamma distribution, and one assumed a lognormal distribution. All studies seemed to modelled treatment effectiveness by applying the hazard ratio of the alternative intervention to the survival function (Online Resource, Appendix 4.3). Lazzaro et al. was unclear about how treatment effectiveness was modelled. ${ }^{39}$ None of the studies mentioned the use of a time-dependent treatment effect. 


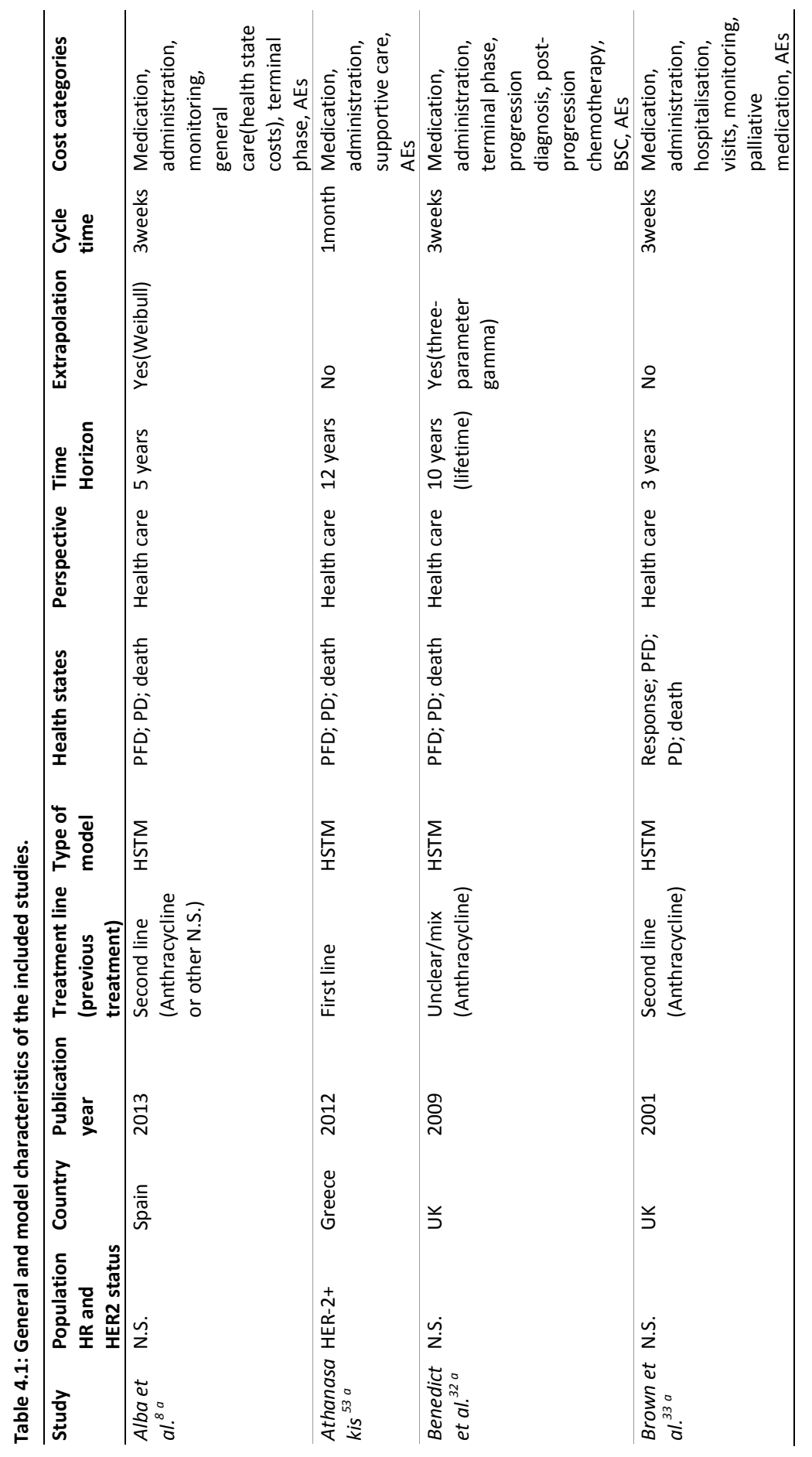




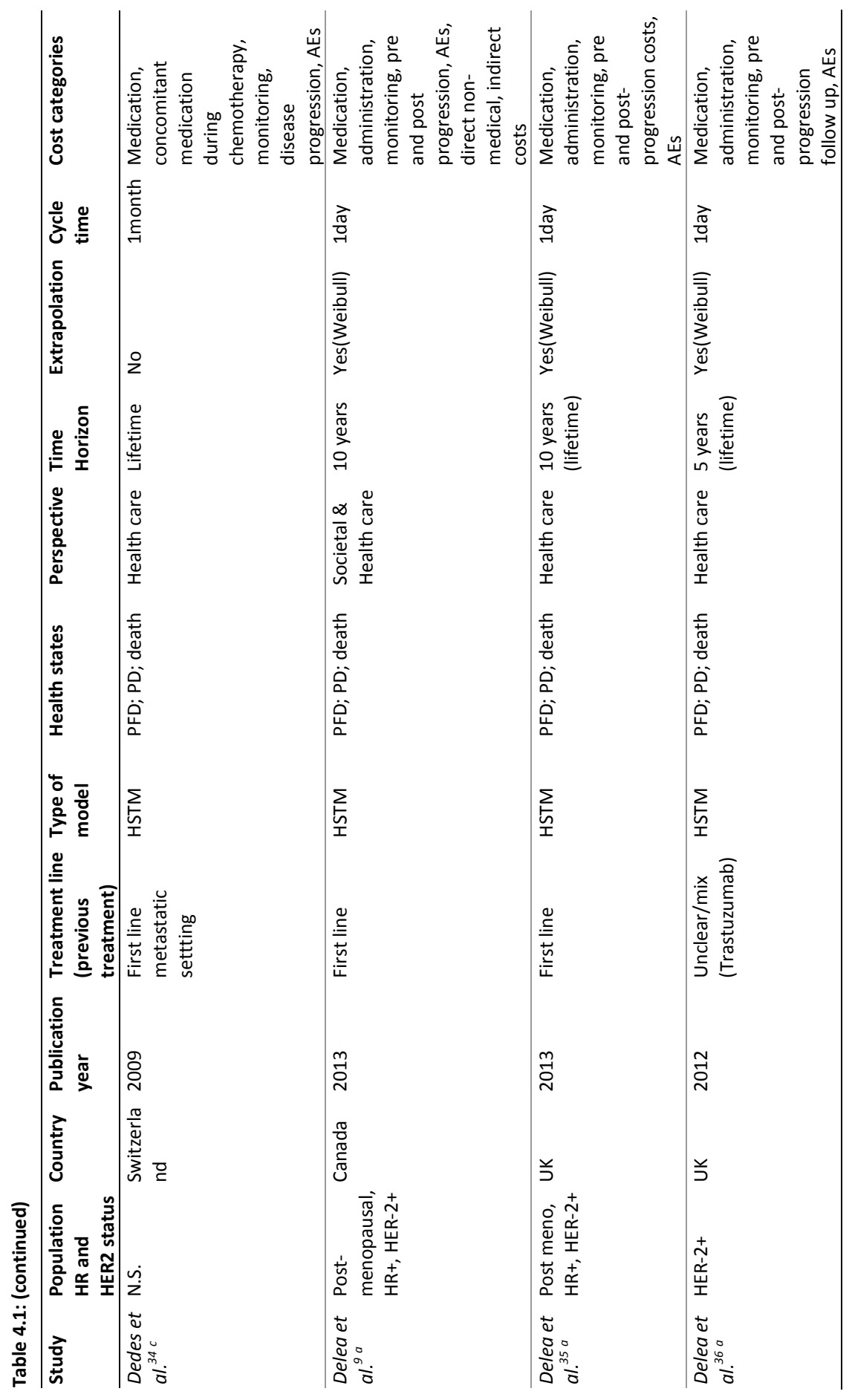




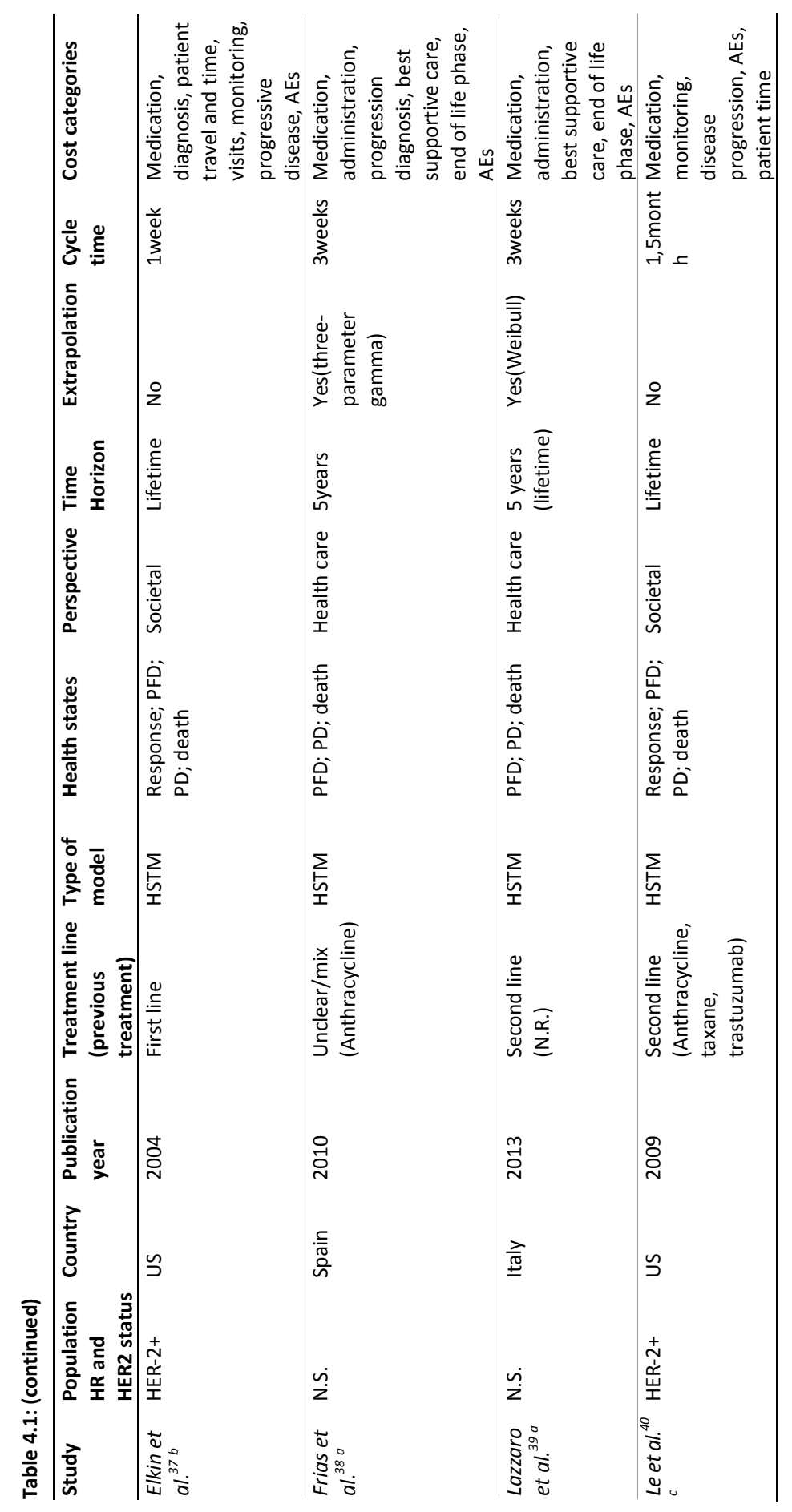




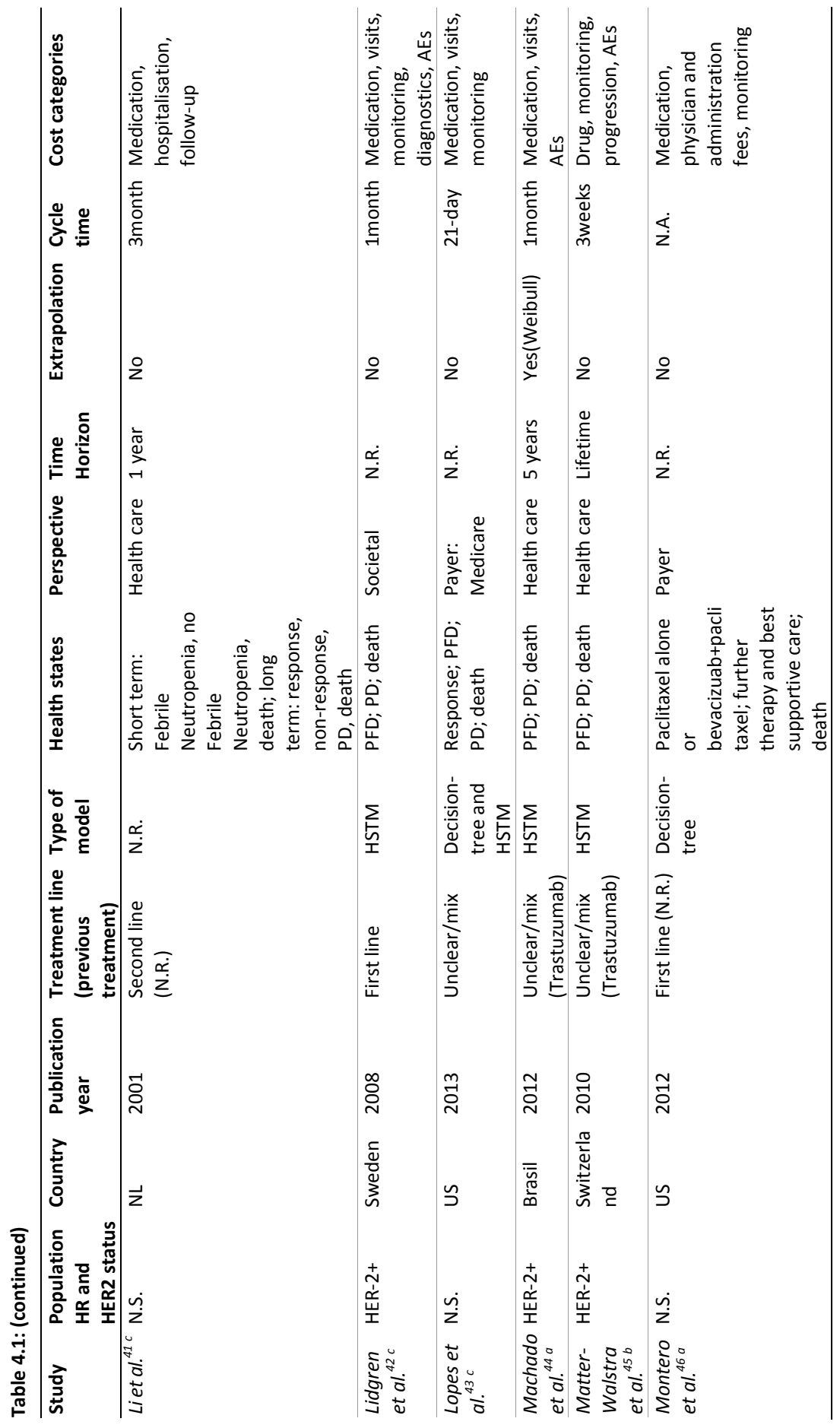




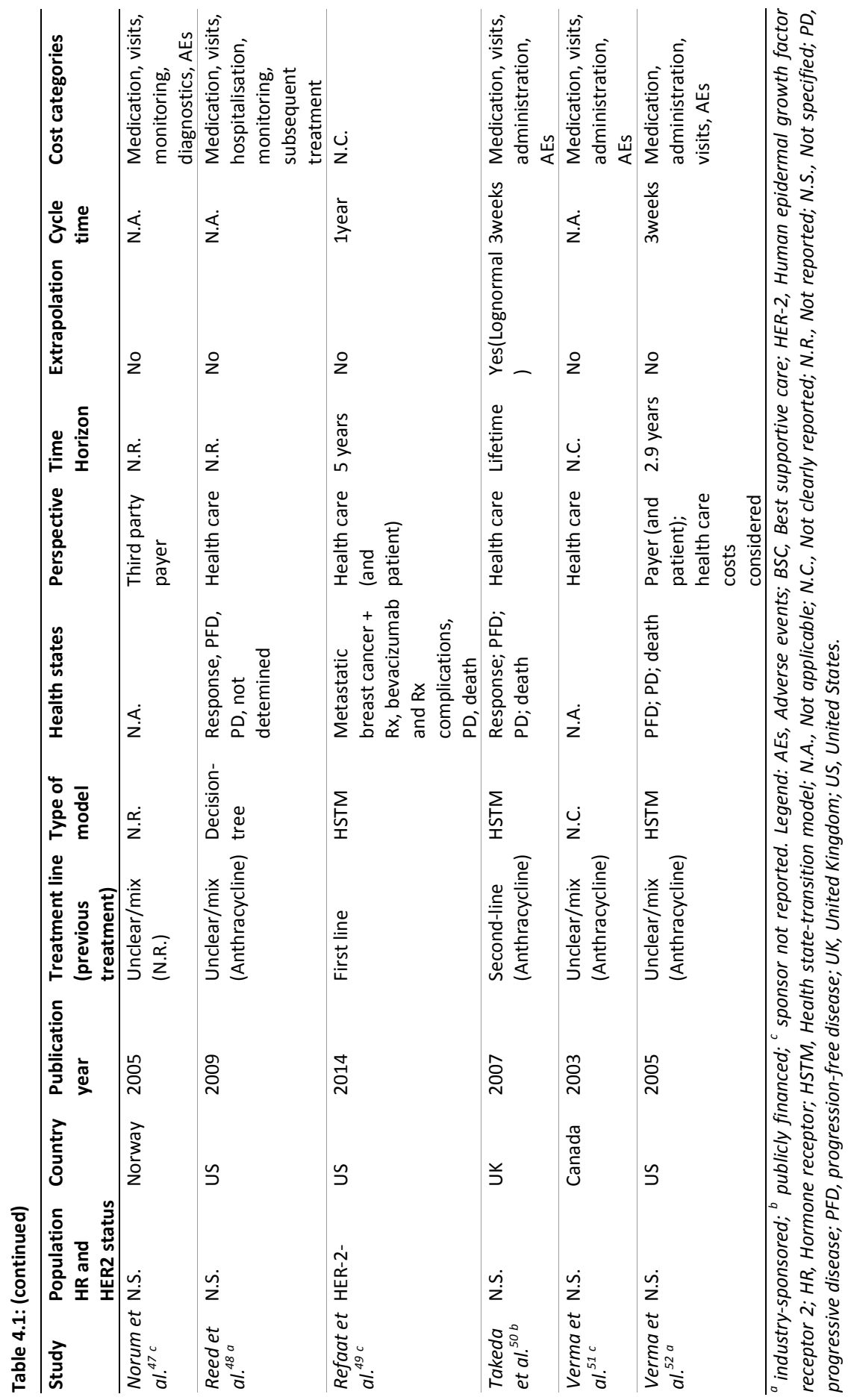


Health state utility values varied from 0.67 to 1.00 , from 0.61 to 0.72 and from 0.26 to 0.68 for the response, stable/progression-free disease and progression health states respectively. Different impact on quality of life were associated with AEs (disutility range: -0.03 to -0.25 ) (Online Resource, Appendix 4.4).

Three studies presented subgroup analyses: one was based on age categories ${ }^{34}$, another on the number of chemotherapy lines received before the interventions under study ${ }^{36}$, the last focused on patients' body mass and surface. ${ }^{45}$

\subsection{Outcomes}

Total LY and QALY ranged from 0.70 to 3.43 and from 0.29 to 2.64 respectively. Total costs ranged from $€ 1,983$ to $€ 86,174$. The NMBs ranged from $€-45,374$ to $€ 59,161$ $(\mathrm{N}=61)$ (Online Resource, Appendix 4.5). Incremental LY and QALY gained varied from 0.06 to 0.74 and from 0.05 to 0.60 respectively. In two comparisons, the intervention dominated the comparator ${ }^{36,44}$, and the intervention (extendedly) dominated the comparator in six comparisons. ${ }^{37,42}$ For the remaining comparisons, the ICERs varied between $€ 200$ and $€ 164,000$ per LY gained $(N=24)$ and between $€ 300$ to $€ 625,000$ per QALY gained ( $N=40)$. The iNMBs ranged from $€-78,574$ to $€ 15,890(N=48), 15$ (31\%) of these iNMBs were positive. Norum et al. ${ }^{47}$ results are not included in this overview because it reported a range of ICERs per LY gained instead of the results of a basecase analysis (Table 4.2).

\subsection{Quality assessment}

Most of the studies clearly described their objective ( $N=16,67 \%)$, comparators $(N=21$; $88 \%)$ and model assumptions ( $\mathrm{N}=22 ; 92 \%)$. A societal perspective was used in four studies (17\%). It was unclear whether the model was appropriate for the decision problem in three studies ( $\mathrm{N}=3 ; 13 \%)$. In two of these studies, the model was not graphically represented and the possible transition between health states were not clearly described. ${ }^{39,52}$ In the third study, all health states of the model were neither mentioned, nor graphically represented $(\mathrm{N}=1,4 \%){ }^{41}$ This hampered the authors in assessing whether the model was appropriate for the decision problem. In two studies $(8 \%)$, the model structure was not considered appropriate given the information provided. The first study did not consider costs incurred after disease progression and did not justify this choice. ${ }^{47}$ The second study considered "patients who died before response could be evaluated and patients for whom the information required for the assessment was not available" as one of the possible response to treatment (i.e. 'Undetermined response'). ${ }^{48}$ 


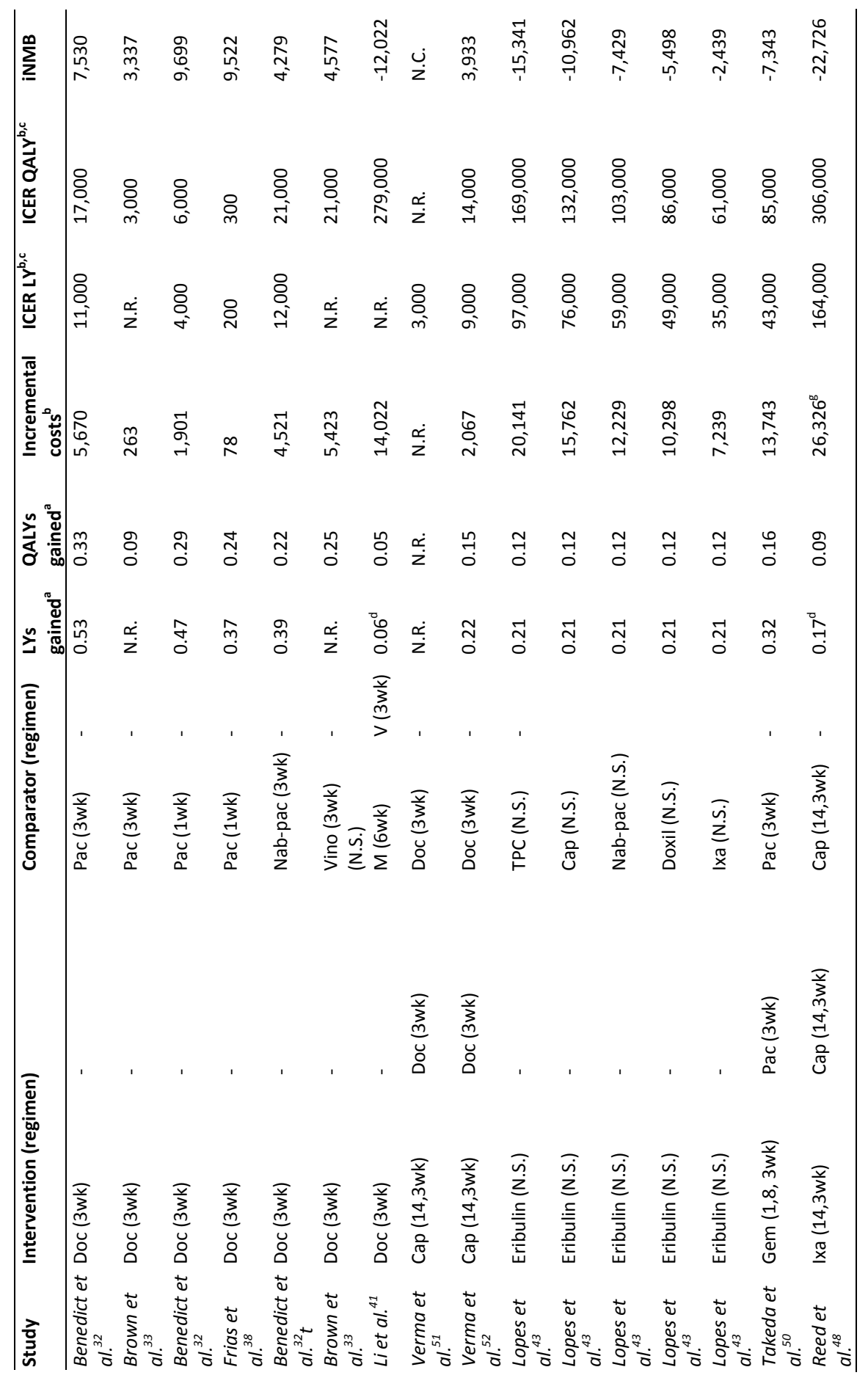




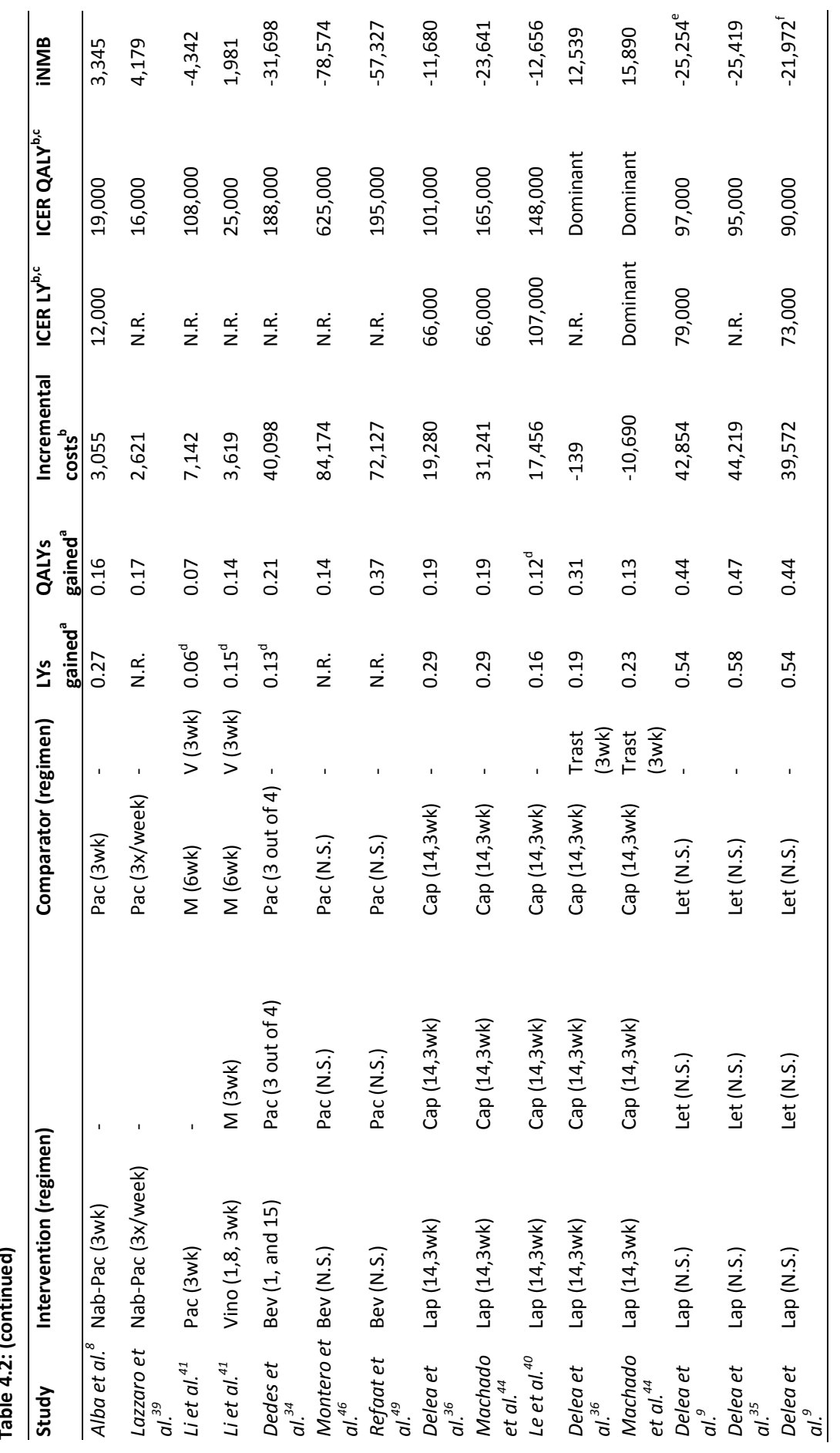




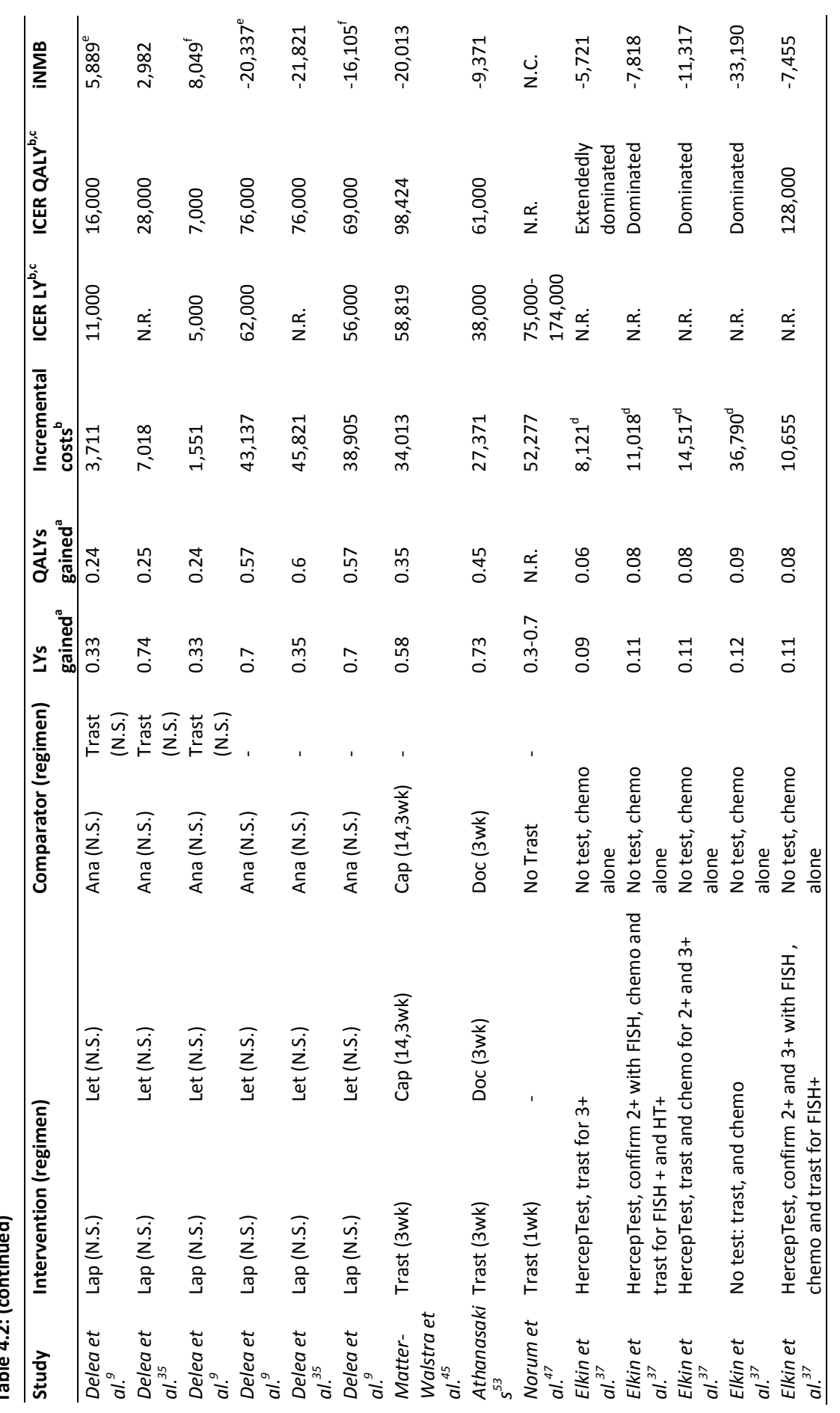




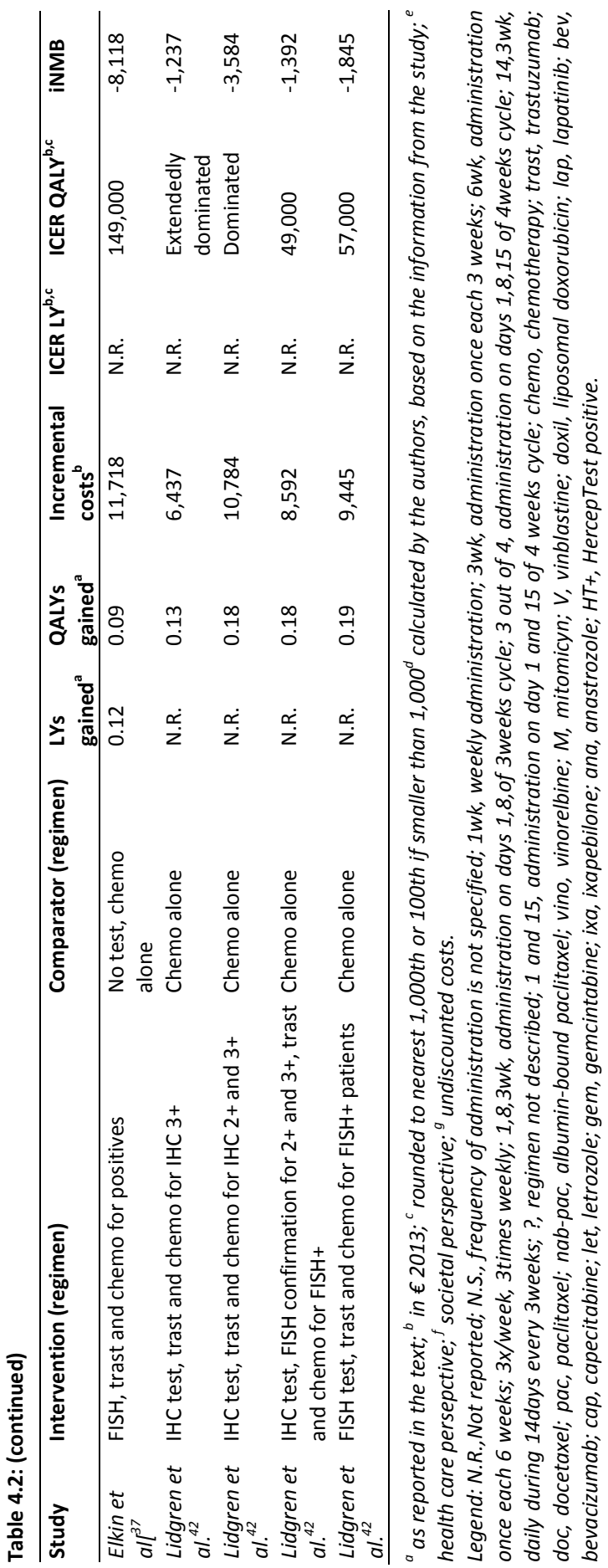


Twenty-one (88\%) studies identified all relevant outcomes and thirteen (54\%) clearly stated the probabilities that outcomes would happen. Outcome measurement and valuation were not clearly described in 13 studies (54\%). Thirteen (54\%) studies clearly identified all important and relevant costs, eighteen (75\%) correctly valued costs and seventeen (71\%) appropriately discounted costs. Fifteen studies (63\%) did not clearly describe how costs were measured. The authors were not able to assess the credibility and accuracy of the sources of all values in ten studies (42\%) because there were not clearly reported. Deterministic and probabilistic sensitivity analyses were performed in $23(96 \%)$ and $17(71 \%)$ studies respectively. Ethical and distributional issues were considered in one study (4\%). None of the studies appropriately fulfilled all items of the quality assessment. Studies fulfilled on average 14 out of the 26 items of the checklist (range 7-20) (Online Resource, Appendix 4.6).

\subsection{Association of model characteristics with study quality and outcomes}

Study quality did not increase over time and did not seem to be associated with study sponsorship and outcomes (Figure 4.2). Recently published studies more often investigated the cost-effectiveness of TT which led to higher iQALY (Figure 4.3).
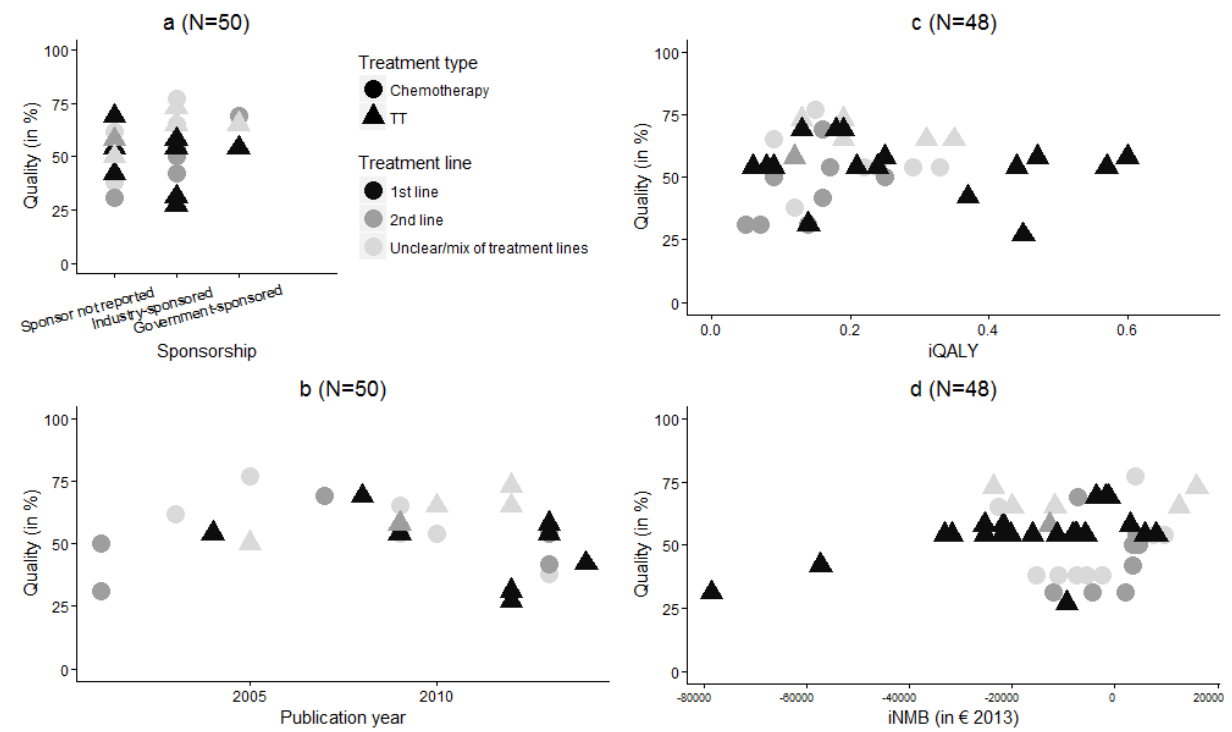

Figure 4.2: Association between study quality and study characteristics and between study quality and outcomes.

Legend: (a) Association between study quality and study sponsorship; (b) Association between study quality and publication year; (c) Association between study quality and IQALY; (d) Association between study quality and $i N M B$; $i Q A L Y$, incremental quality-adjusted life-year; iNMB, incremental net monetary benefit. 

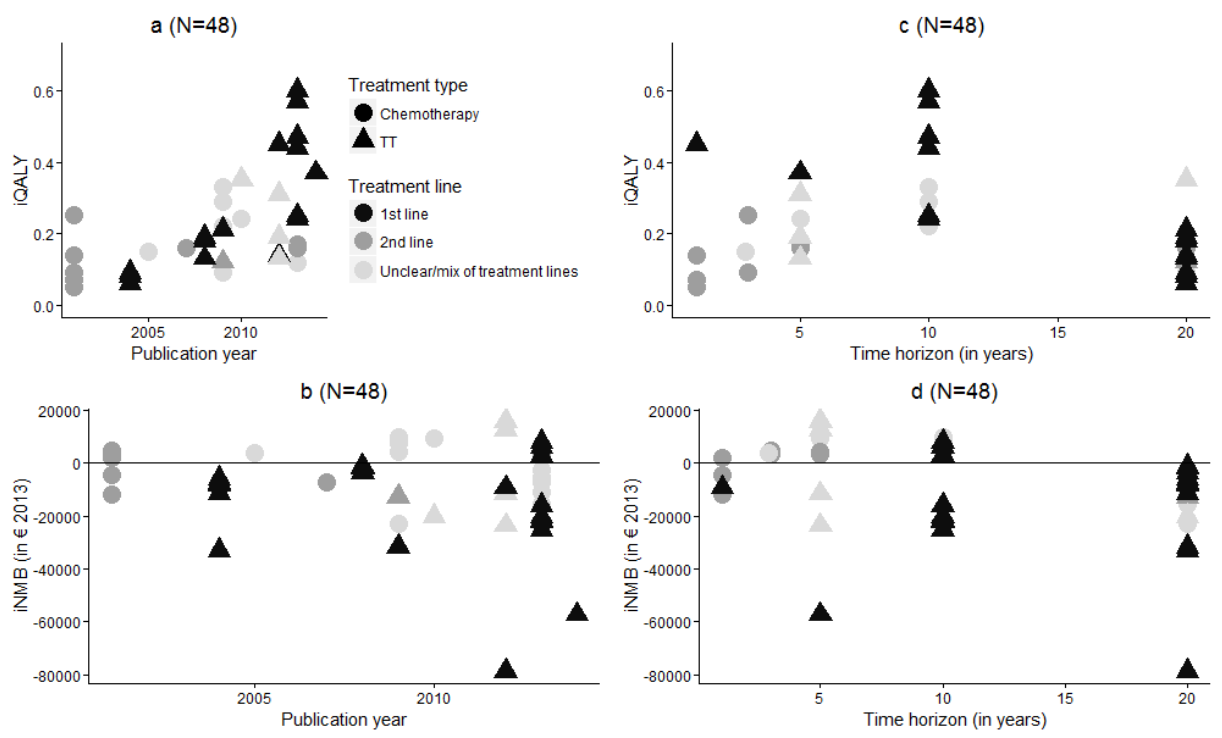

Figure 4.3: Association between model characteristics and study outcomes.

Legend: (a) Association between IQALY and publication year; (b) Association between study iNMB and publication year; (c) Association between $I Q A L Y$ and time horizon; (d) Association between iNMB and time horizon; $i Q A L Y$, incremental quality-adjusted life-year; iNMB, incremental net monetary benefit.

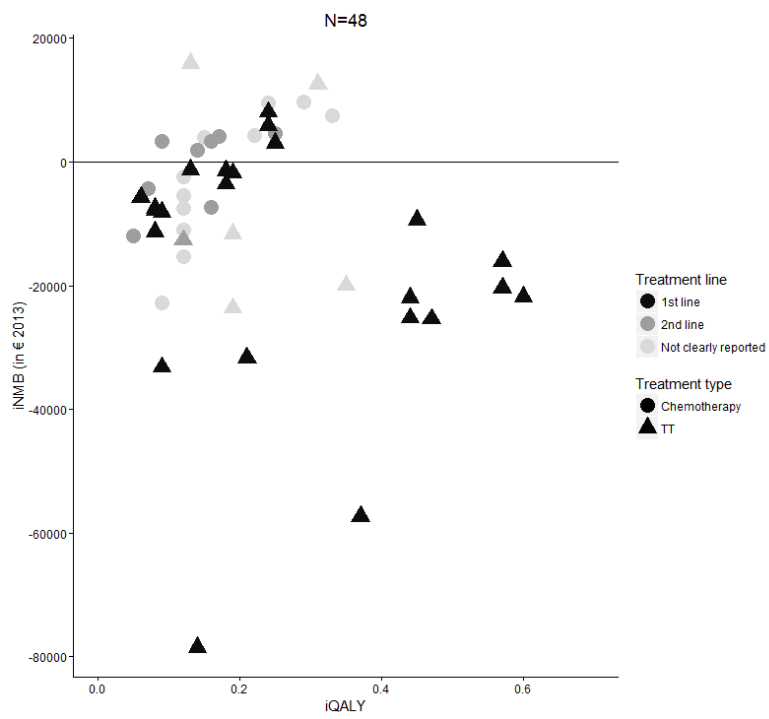

Figure 4.4: Association between iQALYs and iNMBs.

Abbreviations: $I Q A L Y$, incremental quality-adjusted life-year; iNMB, incremental net monetary benefit. 
Lifetime time horizon did not seem to lead to higher health benefits (Figure 4.3). Fourteen out of the twenty-five (56\%) industry-sponsored iNMBs were positive, while one of the twenty-three (4\%) non-industry sponsored iNMBs (sponsorship not reported or governmental sponsorship) was positive. Finally, increased iQALY seemed to be associated with lower iNMB (Figure 4.4).

\section{Discussion}

The current literature review included 24 studies evaluating the cost-effectiveness of chemotherapy or TT for MBC treatment. Most studies (75\%) used a health state transition approach with three health states (stable/progression-free, progression and death) to model $\mathrm{MBC}$, but differed with respect to time horizon, cycle times, AEs and utility values incorporated in the model. Quality of the studies was low and did not increase over time. ILY and IQALY gained ranged between 0.06 and 0.74 and 0.05 and 0.60 respectively. The iNMBs ranged from $€-78,574$ to $€ 15,890,31 \%$ of the iNMBs were positive. TT led to higher IQALY gained. Industry-sponsored studies seemed to result in more favourable iNMB. Larger health benefits were not associated with higher value for money.

The results of the current literature study are subject to certain limitations. Firstly, the literature search was limited in time, publication type and language to make the number of included studies manageable and to retrieve up-to-date assessments potentially using state-of-the-art methodologies. Secondly, an adapted CHEC checklist, which was not specifically developed for model-based economic evaluations, was used for the quality assessment. However, this limitation is unlikely to influence our conclusions because more extensive checklists would also have identified the lack of transparency in reporting. Finally, the small number of studies investigating the same comparisons hampered comparisons of outcomes in relation to differences in model structure (e.g. number of health states) and model inputs. As a result, the consistency in outcomes between different comparisons could not be investigated.

The current study did not demonstrate an association between study quality and study outcomes or sponsorship. While this lack of association is reassuring, the absence of association between study quality and time, mainly due to transparency issues, is worrisome, especially because different guidelines concerning good modeling practices and reporting have been issued. ${ }^{4,54}$ Transparency is a hallmark of good modeling practices because it improves the ability to interpret results and it allows to examine the validity of the models and to reproduce model outcomes. ${ }^{4}$ 
Reproducibility being an essential feature of medical research, (compulsory) disclosure of all model characteristics should be encouraged.

The development of a disease-specific reference model is another solution to resolve consistency, transparency and quality issues. Several authors have already attempted to develop a reference model for MBC treatments. These models were however limited to a specific setting or patient population. ${ }^{19,20}$ Moreover, diseasespecific reference models would avoid duplication of work across jurisdictions and potentially accelerate coverage decision-making for $\mathrm{MBC}$ treatments. It would furthermore decrease the methodological uncertainty associated with different modeling choices made during the cost-effectiveness assessment of MBC treatments.

Increased health benefits did not lead to higher value for money, which implies that treatment costs increased when health benefits became larger. This mechanism is typical of value-based pricing frameworks. However, one might expect that prices would be set in order to remain around the willingness-to-pay threshold in a valuebased pricing setting. This was not the case in the current study, i.e. $31 \%$ of the iNMBs were positive. This might indicate that value-based pricing might be on its way in this field, but that lower prices are needed in order to meet the willingness-to-pay threshold. On the other hand, assessing the value for money of treatments in the metastatic setting only is misleading because using these treatments in the adjuvant setting ${ }^{55}$ or using them more efficiently (e.g. because experience has been acquired in clinical practice) might provide better value for money. The potential value for money of these treatments over their entire life-cycle may be underestimated by only assessing their value in the metastatic setting.

In conclusion, model inputs were highly variable and the quality of the included studies was low, mainly because of a lack of transparency in reporting. The development of a disease-specific reference model would increase the consistency and ensure a minimal quality of cost-effectiveness assessments for $\mathrm{MBC}$ treatments. Cost-effectiveness results were highly variable but, in general, MBC treatments did not provide good value for money. There was no association between study quality and study outcomes. Industry-sponsored studies resulted more often in beneficial value for money of treatments compared to non-industry-sponsored studies. TT led to larger health benefits. Incremental health benefits increased over time, but were outweighed by the increased treatment costs. Consequently, increased health benefits led to lower value for money. 


\section{References}

1. Jackson $\mathrm{CH}$, Sharples LD, Thompson SG. Structural and parameter uncertainty in Bayesian costeffectiveness models. Journal of the Royal Statistical Society: Series C (Applied Statistics). 2010;59(2):233-253.

2. Foster TS, Miller JD, Boye ME, et al. The economic burden of metastatic breast cancer: A systematic review of literature from developed countries. Cancer Treatment Reviews. 2011;37(6):405-415.

3. Drummond M, McGuire A. Economic evaluation in health care: merging theory with practice. Oxford: Oxford University Press; 2001.

4. Eddy DM, Hollingworth W, Caro JJ, et al. Model transparency and validation: a report of the ISPORSMDM Modeling Good Research Practices Task Force-7. Med Decis Making. 2012;32(5):733-743.

5. Penaloza Ramos MC, Barton P, Jowett S, et al. A Systematic Review of Research Guidelines in Decision-Analytic Modeling. Value Health. 2015;18(4):512-29.

6. Frederix GW, van Hasselt JG, Schellens $\mathrm{JH}$, et al. The impact of structural uncertainty on costeffectiveness models for adjuvant endocrine breast cancer treatments: the need for disease-specific model standardization and improved guidance. PharmacoEconomics. 2014;32(1):47-61.

7. Dranitsaris G, Verma S, Trudeau M. Cost utility analysis of first-line hormonal therapy in advanced breast cancer: comparison of two aromatase inhibitors to tamoxifen. Am J Clin Oncol. 2003;26(3): 289-296.

8. Alba E, Ciruelos E, Lopez R, et al. Cost-utility analysis of nanoparticle albumin-bound paclitaxel versus paclitaxel in monotherapy in pretreated metastatic breast cancer in Spain. Expert Rev Pharmacoecon Outcomes Res. 2013;13(3):381-91.

9. Delea T, Amdahl J, Chit A, et al. Cost-effectiveness of lapatinib plus letrozole in her2-positive, hormone receptor-positive metastatic breast cancer in Canada. Curr Oncol. 2013;20(5):e371.

10. Le QA. Structural Uncertainty of Markov Models for Advanced Breast Cancer: A Simulation Study of Lapatinib. Med Decis Making. 2016;36(5):629-640.

11. Bell CM, Urbach DR, Ray JG, et al. Bias in published cost effectiveness studies: systematic review. BMJ. 2006;332(7543):699-703.

12. Greenberg D, Earle C, Fang $\mathrm{CH}$, et al. When is cancer care cost-effective? A systematic overview of cost-utility analyses in oncology. J Natl Cancer Inst. 2010;102(2):82-88.

13. Blank PR, Dedes KJ, Szucs TD. Cost effectiveness of cytotoxic and targeted therapy for metastatic breast cancer: a critical and systematic review. PharmacoEconomics. 2010;28(8):629-47.

14. Diaby V, Tawk R, Sanogo V, et al. A review of systematic reviews of the cost-effectiveness of hormone therapy, chemotherapy, and targeted therapy for breast cancer. Breast Cancer Research Treat. 2015; 151(1):27-40.

15. Afzali HH, Karnon J, Merlin T. Improving the accuracy and comparability of model-based economic evaluations of health technologies for reimbursement decisions: a methodological framework for the development of reference models. Medical decision making : an international journal of the Society for Med Decis Making. 2013;33(3):325-32.

16. Eunethta. Training material on the HTA Core Model 2016 [cited 2016 21-10-2016]. Available from: http://www.eunethta.eu/outputs/training-material-hta-core-model

17. Frederix GW, Severens JL, Hovels AM, et al. The cloudy crystal ball of cost-effectiveness studies. Value Health. 2013;16(6):1100-1102.

18. Eunethta. HTA Core Model ${ }^{\circledR} 2017$ [cited 2017 02-02-2017]. Available from: http://meka.thl.fi/ htacore/BrowseModel.aspx

19. Beauchemin C, Letarte N, Mathurin K, et al. A global economic model to assess the cost-effectiveness of new treatments for advanced breast cancer in Canada. J Med Econ. 2016:1-24.

20. Diaby V, Ali AA, Adunlin G, et al. Parameterization of a disease progression simulation model for sequentially treated metastatic HER2-positive breast cancer patients. Curr Med Res Opin. 2016:1-25.

21. Hind D, Ward S, De Nigris E, et al. Hormonal therapies for early breast cancer: systematic review and economic evaluation. Health Technol Assess. 2007;11(26):iii-iv, ix-xi, 1-134. 
22. Benedict A, Brown RE. Review of cost-effectiveness analyses in hormonal therapies in advanced breast cancer. Expert Opin Pharmacother. 2005;6(11):1789-1801.

23. Frederix GWJ, Severens JL, Hövels AM, et al. Reviewing the Cost-Effectiveness of Endocrine Early Breast Cancer Therapies: Influence of Differences in Modeling Methods on Outcomes. Value Health. 2012;15(1):94-105.

24. Karnon J. Aromatase inhibitors in breast cancer: a review of cost considerations and cost effectiveness. PharmacoEconomics. 2006;24(3):215-232.

25. Eurostat. Consumer Price Index 2013 [cited 2015 21.01.2015]. Available from: http://appsso.eurostat.ec.europa.eu/nui/show.do?dataset=prc_hicp_aind\&lang=en

26. OECD. Consumer Price Index 2013 [cited 2015 21.01.2015]. Available from: http://stats.oecd.org/Index.aspx?querytype=view\&queryname=221\#

27. Statcan. Consumer Price Index 2013 [cited 2015 21.01.2015]. Available from: http://www.statcan.gc.ca/tables-tableaux/sum-som/I01/cst01/econ46a-eng.htm

28. OECD. Purchase Power Parity 2013 [cited 2015 21.01.2015]. Available from: http://stats.oecd.org/Index.aspx?datasetcode=SNA_TABLE4\#

29. Evers S, Goossens $\mathrm{M}$, de Vet $\mathrm{H}$, et al. Criteria list for assessment of methodological quality of economic evaluations: Consensus on Health Economic Criteria. Int J Technol Assess Health Care. $2005 ; 21(2): 240-5$.

30. Soto J. Health economic evaluations using decision analytic modeling. Principles and practices-utilization of a checklist to their development and appraisal. Int J Technol Assess Health Care. 2002;18(1):94-111.

31. Fleeman N, Bagust A, Boland A, et al. Lapatinib and trastuzumab in combination with an aromatase inhibitor for the first-line treatment of metastatic hormone receptor-positive breast cancer which over-expresses human epidermal growth factor 2 (HER2): a systematic review and economic analysis. Health Technol Assess. 2011;15(42):1-93, iii-iv.

32. Benedict A, Cameron DA, Corson $\mathrm{H}$, et al. An economic evaluation of docetaxel and paclitaxel regimens in metastatic breast cancer in the UK. PharmacoEconomics. 2009;27(10):847-859.

33. Brown RE, Hutton J, Burrell A. Cost effectiveness of treatment options in advanced breast cancer in the UK. PharmacoEconomics. 2001;19(11):1091-1102.

34. Dedes KJ, Matter-Walstra K, Schwenkglenks M, et al. Bevacizumab in combination with paclitaxel for HER-2 negative metastatic breast cancer: an economic evaluation. Eur J Cancer. 2009;45(8): 1397-1406.

35. Delea TE, Hawkes C, Amonkar MM, et al. Cost-Effectiveness of Lapatinib plus Letrozole in PostMenopausal Women with Hormone Receptor-and HER2-Positive Metastatic Breast Cancer. Breast Care. 2013;8(6):429-437.

36. Delea TE, Tappenden P, Sofrygin O, et al. Cost-effectiveness of lapatinib plus capecitabine in women with HER2 + metastatic breast cancer who have received prior therapy with trastuzumab. Eur J Health Econ. 2012;13(5):589-603.

37. Elkin EB, Weinstein MC, Winer EP, et al. HER-2 testing and trastuzumab therapy for metastatic breast cancer: a cost-effectiveness analysis. J Clin Oncol. 2004;22(5):854-863.

38. Frias C, Cortes J, Segui MA, et al. Cost-effectiveness analyses of docetaxel versus paclitaxel once weekly in patients with metastatic breast cancer in progression following anthracycline chemotherapy, in Spain. Clin Transl Oncol. 2010;12(10):692-700.

39. Lazzaro C, Bordonaro R, Cognetti F, et al. An Italian cost-effectiveness analysis of paclitaxel albumin (nab-paclitaxel) versus conventional paclitaxel for metastatic breast cancer patients: the COSTANza study. ClinicoEconomics and outcomes research: CEOR. 2013;5:125.

40. Le QA, Hay JW. Cost-effectiveness analysis of lapatinib in HER-2-positive advanced breast cancer. Cancer. 2009;115(3):489-498.

41. Li N, van Agthoven $\mathrm{M}$, Willemse $\mathrm{P}$, et al. A cost--utility analysis comparing second-line chemotherapy schemes in patients with metastatic breast cancer. Anticancer Ddrugs. 2001;12(6):533-540.

42. Lidgren $\mathrm{M}$, Wilking $\mathrm{N}$, Jonsson $\mathrm{B}$, et al. Cost-effectiveness of HER2 testing and trastuzumab therapy for metastatic breast cancer. Acta Oncol. 2008;47(6):1018-28. 
43. Lopes G, Gluck S, Avancha K, et al. A cost effectiveness study of eribulin versus standard single-agent cytotoxic chemotherapy for women with previously treated metastatic breast cancer. Breast Cancer Res Treat. 2013;137(1):187-93.

44. Machado M, Einarson TR. Lapatinib in patients with metastatic breast cancer following initial treatment with trastuzumab: an economic analysis from the Brazilian public health care perspective. Breast Cancer: Targets Ther. 2012;4:173.

45. Matter-Walstra KW, Dedes KJ, Schwenkglenks M, et al. Trastuzumab beyond progression: a costutility analysis. Ann Oncol. 2010;21(11):2161-2168..

46. Montero AJ, Avancha K, Gluck S, et al. A cost-benefit analysis of bevacizumab in combination with paclitaxel in the first-line treatment of patients with metastatic breast cancer. Breast Cancer Res Treat. 2012;132(2):747-751.

47. Norum J, Risberg T, Olsen JA. A monoclonal antibody against HER-2 (trastuzumab) for metastatic breast cancer: a model-based cost-effectiveness analysis. Ann Oncol. 2005;16(6):909-914.

48. Reed SD, Li Y, Anstrom KJ, et al. Cost effectiveness of ixabepilone plus capecitabine for metastatic breast cancer progressing after anthracycline and taxane treatment. J Clin Oncol. 2009;27(13): 2185-2191.

49. Refaat T, Choi M, Gaber G, et al. Markov Model and Cost-Effectiveness Analysis of Bevacizumab in HER2-Negative Metastatic Breast Cancer. Am J Clin Oncol. 2014;37(5):480-485.

50. Takeda AL, Jones J, Loveman E, et al. The clinical effectiveness and cost-effectiveness of gemcitabine for metastatic breast cancer: a systematic review and economic evaluation. Health Technol Assess. 2007;11(19):iii, ix-xi, 1-62.

51. Verma S, llersich AL. Population-based pharmacoeconomic model for adopting capecitabine/docetaxel combination treatment for anthracycline-pretreated metastatic breast cancer. Oncologist. 2003;8(3):232-240.

52. Verma S, Maraninchi D, O'Shaughnessy J, et al. Capecitabine plus docetaxel combination therapy. Cancer. 2005;103(12):2455-2465.

53. Athanasakis K, J K. A cost-effectiveness analysis of trastuzumab plus docetaxel vs. docetaxel alone for the treatment of HER2-positive metastatic breast cancer in the Greek healthcare setting. Forum of Clinical Oncology. 2012;2012(3):4.

54. Husereau D, Drummond M, Petrou S, et al. Consolidated Health Economic Evaluation Reporting Standards (CHEERS) statement. Int J Technol Assess Health Care. 2013;29(2):117-122.

55. Dvortsin E, Gout-Zwart J, Eijssen EM, et al. Comparative Cost-Effectiveness of Drugs in Early versus Late Stages of Cancer; Review of the Literature and a Case Study in Breast Cancer. PLoS One. 2016;11(1):e0146551. 


\section{Appendices}

Appendix 4.1: Full search query PubMED and NHS EED**

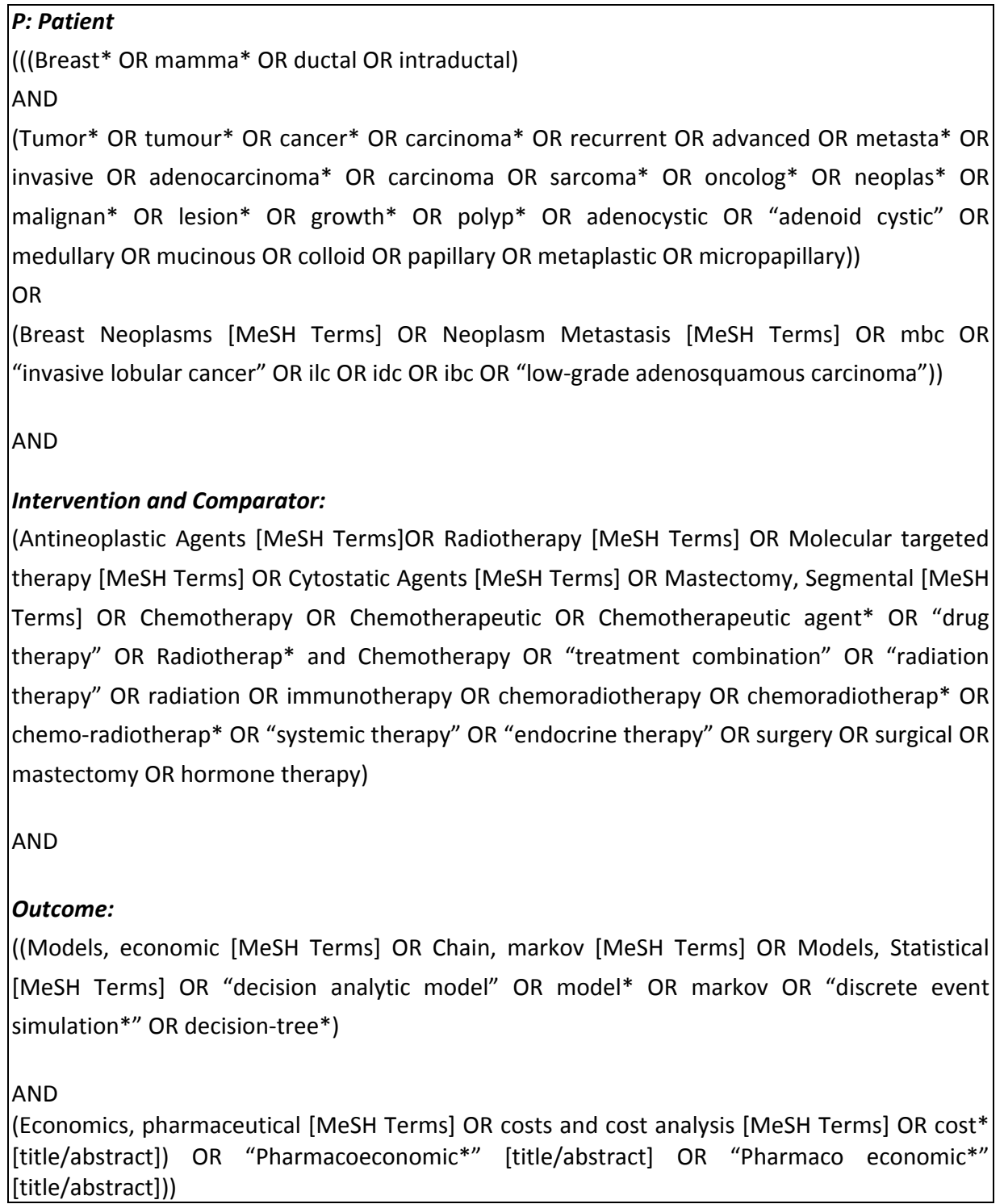

**NHS EED search was limited to the following terms: "Breast cancer" OR "Breast neoplasm", in order to retrieve all relevant studies, since this database only contains economic evaluation. Therefore, the economic evaluation-part of the PubMed search was not necessary. 


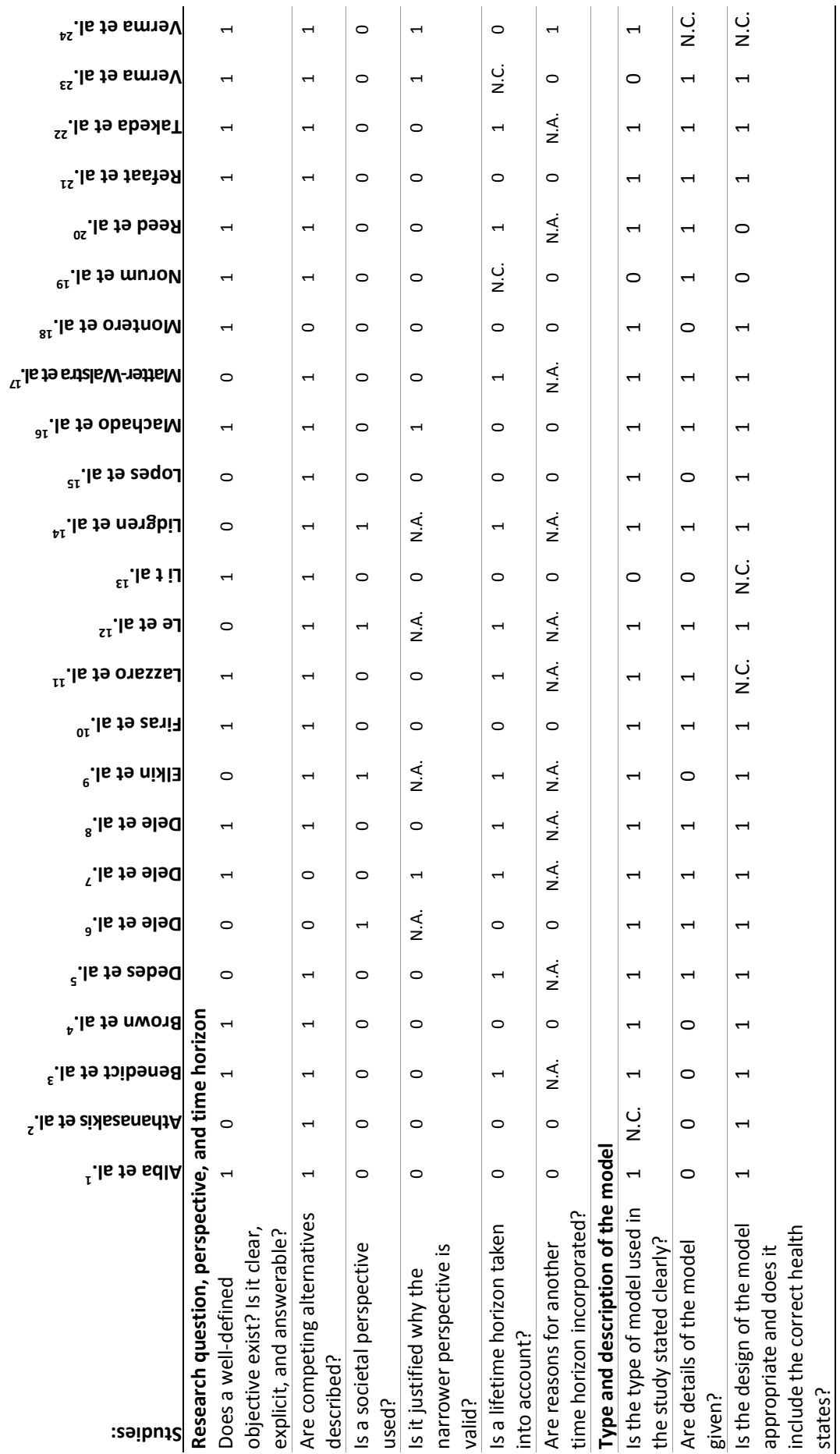




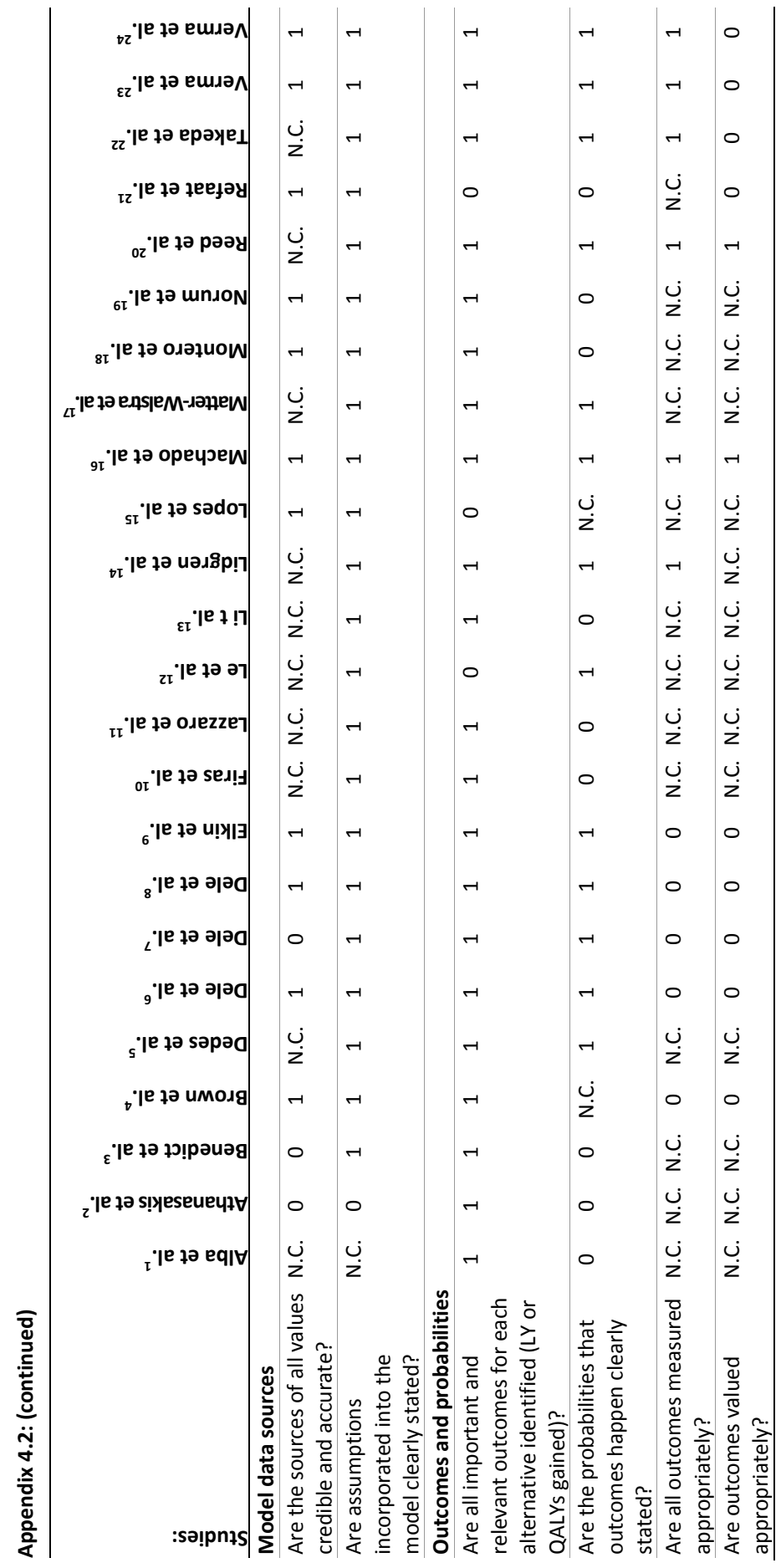




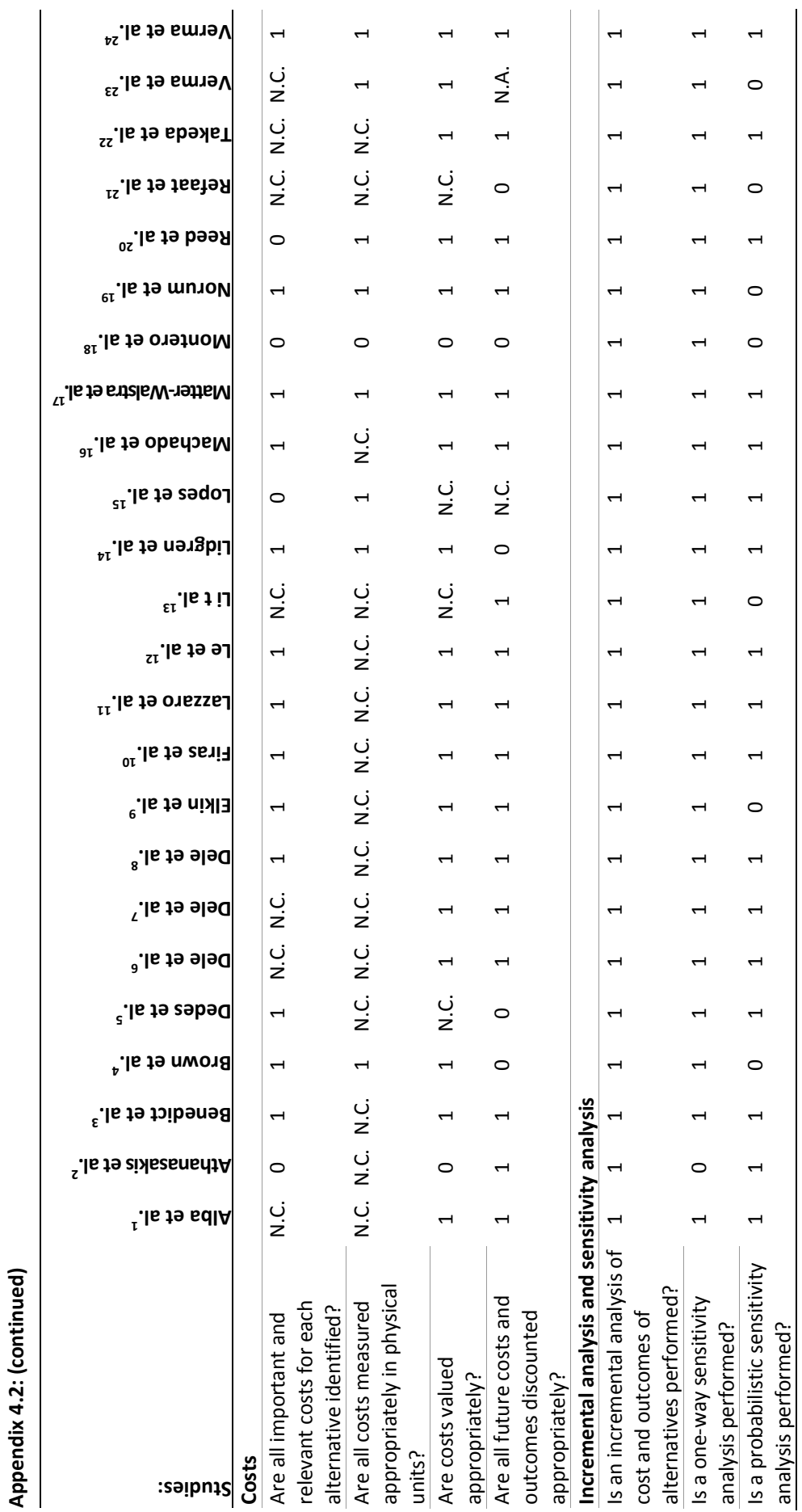




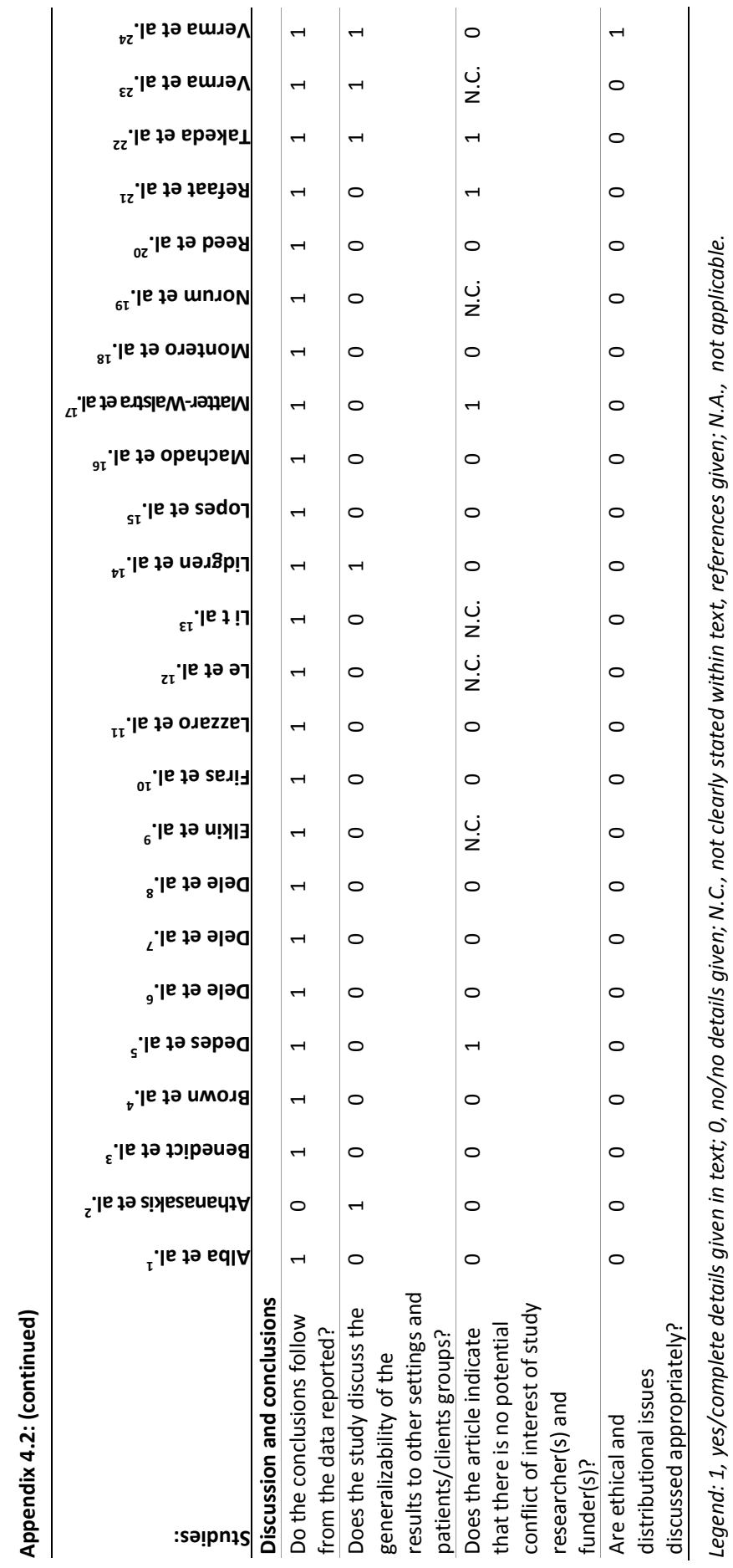


Appendix 4.3: HRs sorted by regimens and studies

\begin{tabular}{|c|c|c|c|c|c|c|c|}
\hline \multirow{2}{*}{$\begin{array}{l}\text { Studies } \\
3\end{array}$} & \multicolumn{2}{|c|}{ Intervention (administration schedule) } & \multicolumn{2}{|l|}{ Comparator } & \multirow{2}{*}{$\begin{array}{l}\text { HR PFS } \\
1.64\end{array}$} & \multirow{2}{*}{$\begin{array}{l}\text { HR OS } \\
1.41\end{array}$} & \multirow{2}{*}{$\begin{array}{l}\text { HR from } \\
\text { progression } \\
\text { to death } \\
\text { - }\end{array}$} \\
\hline & Doc (3wk) & - & Pac (3wk) & - & & & \\
\hline 4 & Doc (3wk) & - & $\operatorname{Pac}(3 w k)$ & - & N.R. & N.R. & - \\
\hline 13 & Doc (3wk) & - & $M(6 w k)$ & $V(3 w k)$ & N.R. & N.R. & - \\
\hline 23 & Cap (14,3wk) & Doc (3wk) & Doc (3wk) & - & 0.652 & 0.777 & - \\
\hline 24 & Cap $(14,3 w k)$ & Doc (3wk) & Doc (3wk) & - & N.R. & 0.775 & - \\
\hline 15 & Eribulin (N.S.) & - & TPC (N.S.) & - & 0.87 & 0.81 & - \\
\hline 22 & $\operatorname{Gem}(1,8,3 w k)$ & Pac (3wk) & $\operatorname{Pac}(3 w k)$ & - & 0.65 & 0.78 & - \\
\hline 20 & Ixa $(14,3 w k)$ & Cap $(14,3 w k)$ & Cap $(14,3 w k)$ & - & N.R. & N.R. & - \\
\hline 1 & Nab-Pac (3wk) & - & $\operatorname{Pac}(3 w k)$ & - & 0.721 & 0.734 & - \\
\hline 3 & Nab-Pac (3wk) & & $\operatorname{Pac}(3 w k)$ & & 1.33 & 1.11 & - \\
\hline 11 & Nab-Pac (3x/week) & - & $\operatorname{Pac}(3 x /$ week) & - & $0.73-0.75^{a}$ & $0.73^{\mathrm{a}}$ & - \\
\hline 13 & $\operatorname{Pac}(3 w k)$ & - & $M(6 w k)$ & $V(3 w k)$ & N.R. & N.R. & - \\
\hline 3 & Pac (1wk) & & $\operatorname{Pac}(3 w k)$ & & 1.09 & 1.04 & - \\
\hline 10 & $\operatorname{Pac}(1 w k)$ & - & Doc (3wk) & - & 1.64 & 1.41 & - \\
\hline 13 & Vino $(1,8,3 w k)$ & $M(3 w k)$ & $\mathrm{M}(6 w k)$ & $V(3 w k)$ & N.R. & N.R. & - \\
\hline 5 & $\operatorname{Bev}(1$, and 15$)$ & Pac (3 out of 4 ) & Pac (3 out of 4 ) & - & $0.6^{\mathrm{b}}$ & $0.88^{b}$ & - \\
\hline 18 & Bev (N.S.) & Pac (N.S.) & Pac (N.S.) & - & N.R. & N.R. & - \\
\hline 21 & Bev (N.S.) & Pac (N.S.) & $\operatorname{Pac}($ N.S.) & - & N.R. & N.R. & - \\
\hline 16 & Lap (14,3wk) & Cap $(14,3 w k)$ & Cap $(14,3 w k)$ & - & 0.55 & - & - \\
\hline 16 & Cap $(14,3 w k)$ & & Lap $(14,3 w k)$ & Cap $(14,3 w k)$ & - & 1.33 & - \\
\hline 12 & Lap (14,3wk) & Cap $(14,3 w k)$ & Cap $(14,3 w k)$ & - & N.A. & N.A. & - \\
\hline 8 & Lap (14,3wk) & Cap $(14,3 w k)$ & Cap $(14,3 w k)$ & - & 0.55 & - & - \\
\hline 8 & Cap $(14,3 w k)$ & & $\operatorname{Lap}(14,3 w k)$ & Cap $(14,3 w k)$ & - & 1.333 & - \\
\hline 6 & Lap (N.S.) & Let (N.S.) & Let (N.S.) & - & 0.65 & 0.77 & - \\
\hline 7 & Lap (N.S.) & Let (N.S.) & Let (N.S.) & - & 0.65 & 0.77 & - \\
\hline 6 & Trast & Ana & Let (N.S.) & - & 0.73 & 0.90 & - \\
\hline 7 & Trast & Ana & Let (N.S.) & - & 0.73 & 0.90 & - \\
\hline 2 & Trast & Doc & Doc & - & N.R. & N.R. & - \\
\hline 6 & Ana & & Let (N.S.) & - & 1.22 & 1.08 & - \\
\hline 7 & Ana & & Let (N.S.) & - & 1.22 & 1.08 & - \\
\hline 8 & Trast (3wk) & Cap $(14,3 w k)$ & Cap $(14,3 w k)$ & - & 0.685 & 0.763 & - \\
\hline 16 & Trast (3wk) & Cap $(14,3 w k)$ & Cap $(14,3 w k)$ & & 0.69 & 0.94 & - \\
\hline 17 & Trast (3wk) & Cap $(14,3 w k)$ & Cap $(14,3 w k)$ & - & 0.69 & N.A. & 1.035 \\
\hline 19 & Trast (1wk) & - & No Trast & - & N.A. & N.A. & - \\
\hline 9 & HercepTest, trast an & emo for $3+$ & No test, chemo & alone & N.R. & N.R. & - \\
\hline 9 & $\begin{array}{l}\text { HercepTest, confirm } \\
\text { trast for FISH + and }\end{array}$ & with FISH, chemo and & No test, chemo & alone & N.R. & N.R. & - \\
\hline 9 & HercepTest, trast an & emo for $2+$ and $3+$ & No test, chemo & alone & N.R. & N.R. & - \\
\hline 9 & No test: trast, and ch & & No test, chemo & alone & N.R. & N.R. & - \\
\hline 9 & $\begin{array}{l}\text { HercepTest, confirm } \\
\text { chemo and trast for }\end{array}$ & and $3+$ with FISH & No test, chemo & alone & N.R. & N.R. & - \\
\hline 9 & FISH, trast and chem & r positives & No test, chemo & alone & N.R. & N.R. & - \\
\hline 14 & $\mathrm{IHC}$ test, trast and $\mathrm{ch}$ & for $\mathrm{IHC} 3+$ & Chemo alone & & N.A. & N.A. & - \\
\hline 14 & IHC test, trast and ch & for IHC $2+$ and $3+$ & Chemo alone & & N.A. & N.A. & - \\
\hline 14 & $\begin{array}{l}\text { IHC test, FISH confir } \\
\text { and chemo for FISH }\end{array}$ & on for $2+$ and $3+$, trast & Chemo alone & & N.A. & N.A. & - \\
\hline 14 & FISH test, trast and & to for FISH+ patients & Chemo alone & & N.A. & N.A. & - \\
\hline
\end{tabular}

${ }^{a}$ reported in introduction; ${ }^{b}$ assuming it is based on ref 4 of the publication (Miller et al., E2100 trial. ${ }^{25]}$

Legend: ana, anastrozole; bev, bevacizumab; cap, capecitabine; chemo, chemotherapy; trast, trastuzumab; doc, docetaxel; doxil, liposomal doxorubicin; FISH, fluorescent in situ hybridization; gem, gemcintabine; HR, hazard ratio; HT+, HercepTest positive; IHC, immunohistochemistry; ixa, ixapebilone; lap, lapatinib; let, letrozole; $M$, mitomicyn; nab-pac, albumin-bound paclitaxel; N.R., not reported; N.S., administration schedule not specified; pac, paclitaxel; PD, progressed disease; PFS, progression-free survival; N.A., not applicable; V, vinblastine; vino, vinorelbine; 1wk, weekly administration; 3wk, administration once each 3 weeks; $6 w k$, administration once each 6 weeks; $3 x /$ week, 3times weekly; 1,8,3wk, administration on days 1,8, of 3weeks cycle; 3 out of 4, administration on days 1,8,15 of 4weeks cycle; 14,3wk, daily during 14days every 3weeks; 1 and 15, administration on day 1 and 15 of 4 weeks cycle. 


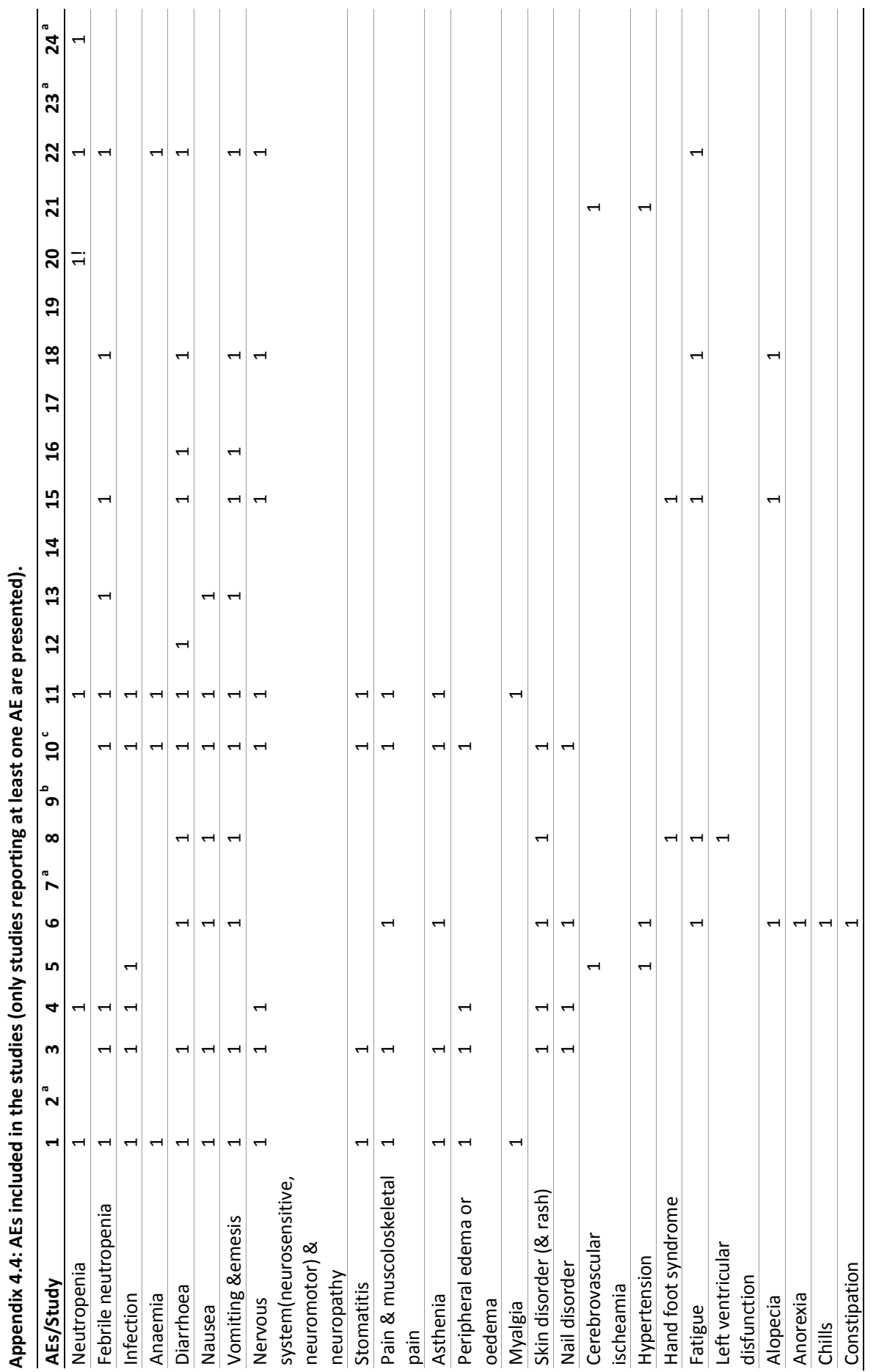




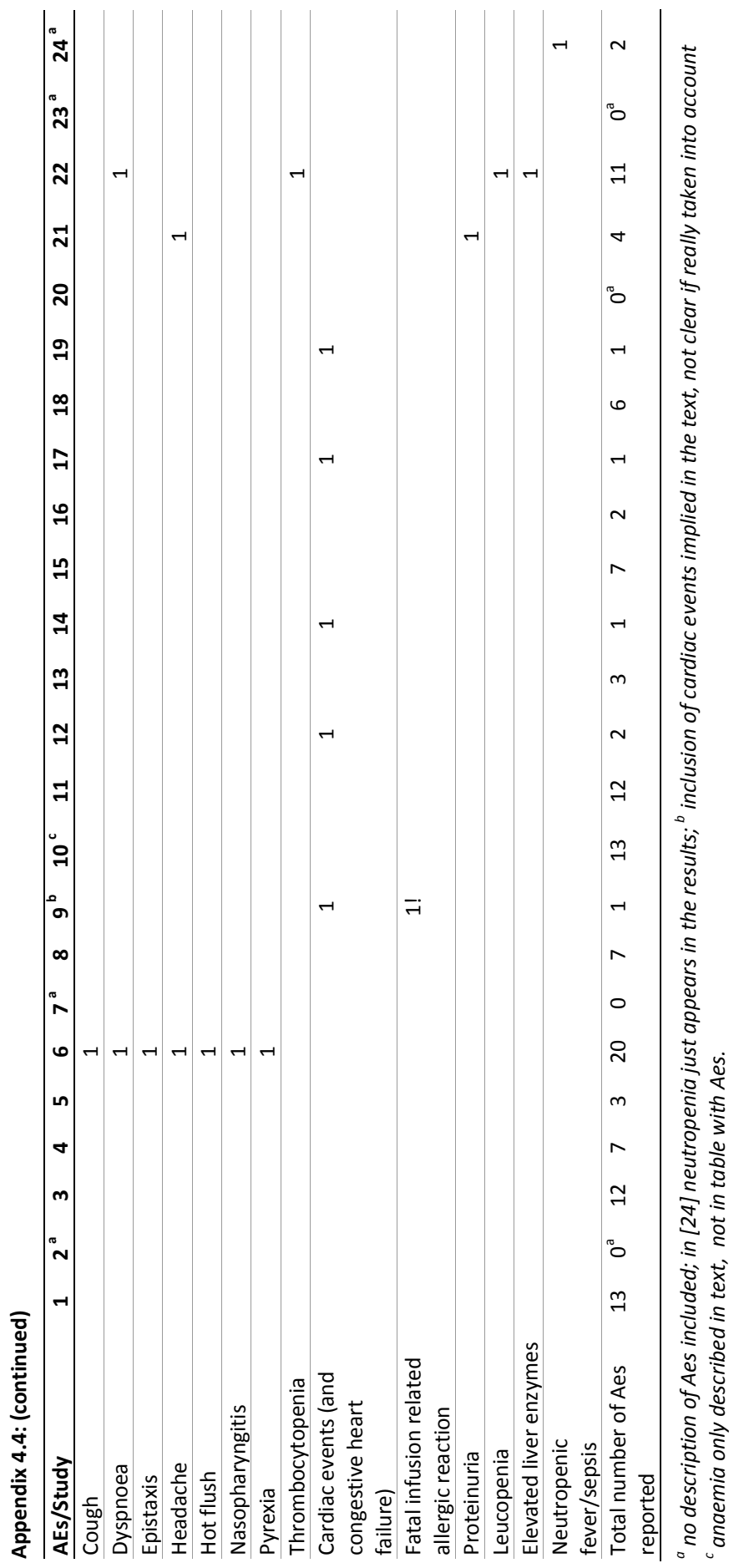


Appendix 4.5: Total cost, QALY, LY and NMB for each comparator

\begin{tabular}{|c|c|c|c|c|c|c|}
\hline Studies & $\begin{array}{l}\text { Publication } \\
\text { year }\end{array}$ & Treatment & $\begin{array}{l}\text { Total } \\
\text { costs }\end{array}$ & $\begin{array}{l}\text { QALY } \\
\text { gained }\end{array}$ & LY gained & $\mathrm{NMB}^{\mathrm{a}}$ \\
\hline 1 & 2013 & Nab-pac (3wk) & 17,104 & 0.80 & 1.44 & 14,896 \\
\hline 1 & 2013 & $\operatorname{Pac}(3 w k)$ & 14,049 & 0.64 & 1.17 & 11,551 \\
\hline 1 & 2013 & $\operatorname{Pac}(1 w k)$ & 17,844 & 0.80 & 1.44 & 14,156 \\
\hline 2 & 2012 & Doc (3wk) & 9,129 & 1.54 & 2.70 & 52,551 \\
\hline 2 & 2012 & Trast+doc (3wk) & 36,505 & 1.99 & 3.43 & 43,175 \\
\hline 3 & 2009 & Nab-pac (3wk) & 19,910 & 0.96 & 1.62 & 18,490 \\
\hline 3 & 2009 & $\operatorname{Pac}(3 \mathrm{wk})$ & 18,761 & 0.85 & 1.48 & 15,239 \\
\hline 3 & 2009 & $\operatorname{Pac}(1 w k)$ & 22,529 & 0.89 & 1.54 & 13,071 \\
\hline 3 & 2009 & Doc (3 wk) & 24,431 & 1.18 & 2.01 & 22,769 \\
\hline 4 & 2001 & $\operatorname{Pac}(3 \mathrm{wk})$ & 11,683 & 0.65 & N.R. & 14,257 \\
\hline 4 & 2001 & Doc (3 wk) & 11,945 & 0.73 & N.R. & 17,443 \\
\hline 5 & 2009 & Pac (3 out of 4 ) & 28,480 & 0.69 & 2.10 & -880 \\
\hline 5 & 2009 & $\operatorname{Pac}(3$ out of 4$)+\operatorname{bev}(1$ and 15$)$ & 68,578 & 0.90 & 2.23 & $-32,578$ \\
\hline $6^{b}$ & 2013 & Lap+let (N.S.) & 69,650 & 2.30 & 3.27 & 22,430 \\
\hline $6^{\mathrm{b}}$ & 2013 & Let (N.S.) & 26,796 & 1.86 & 2.72 & 47,684 \\
\hline $6^{\mathrm{b}}$ & 2013 & Trast+ana (N.S.) & 65,939 & 2.07 & 2.94 & 16,701 \\
\hline $6^{\mathrm{b}}$ & 2013 & Ana (N.S.) & 26,513 & 1.73 & 2.57 & 42,807 \\
\hline$]^{c}$ & 2013 & Lap+let (N.S.) & 79,632 & 2.30 & 3.27 & 12,448 \\
\hline $6^{c}$ & 2013 & Let (N.S.) & 40,060 & 1.86 & 2.72 & 34,420 \\
\hline $6^{c}$ & 2013 & Trast+ana (N.S.) & 78,081 & 2.07 & 2.94 & 4,559 \\
\hline $6^{c}$ & 2013 & Ana (N.S.) & 40,727 & 1.73 & 2.57 & 28,633 \\
\hline 7 & 2013 & Lap+let (N.S.) & 77,160 & 2.39 & 3.40 & 18,400 \\
\hline 7 & 2013 & Let (N.S.) & 32,942 & 1.92 & 2.82 & 43,978 \\
\hline 7 & 2013 & Trast+ana (N.S.) & 70,142 & 2.14 & 2.66 & 15,338 \\
\hline 7 & 2013 & Ana (N.S.) & 31,341 & 1.79 & 3.05 & 40,179 \\
\hline 8 & 2012 & Lap+cap $(14,3 w k)$ & 37,460 & 0.93 & 1.65 & -380 \\
\hline 8 & 2012 & Cap $(14,3 w k)$ & 18,180 & 0.74 & 1.36 & 11,300 \\
\hline 8 & 2012 & Trast $(3 w k)+\operatorname{cap}(14,3 w k)$ & 37,600 & 0.90 & 1.63 & $-1,760$ \\
\hline 9 & 2004 & HercepTest, trast and chemo for $3+$ & 44,428 & 1.28 & 2.34 & 6,772 \\
\hline 9 & 2004 & $\begin{array}{l}\text { HercepTest, confirm } 2+\text { with FISH, } \\
\text { chemo and trast for FISH + and HT+ }\end{array}$ & 52,549 & 1.34 & 2.42 & 1,051 \\
\hline 9 & 2004 & $\begin{array}{l}\text { HercepTest, trast and chemo for } 2+ \\
\text { and } 3+\end{array}$ & 55,084 & 1.36 & 2.44 & -684 \\
\hline 9 & 2004 & No test: trast, and chemo & 55,447 & 1.36 & 2.44 & $-1,047$ \\
\hline 9 & 2004 & $\begin{array}{l}\text { HercepTest, confirm } 2+\text { and } 3+\text { with } \\
\text { FISH , chemo and trast for FISH+ }\end{array}$ & 58,945 & 1.36 & 2.44 & $-4,545$ \\
\hline 9 & 2004 & FISH, trast and chemo for positives & 56,146 & 1.37 & 2.45 & $-1,346$ \\
\hline 9 & 2004 & No test, trast, and chemo & 81,218 & 1.37 & 2.45 & $-26,418$ \\
\hline 10 & 2010 & $\operatorname{Pac}(1 w k)$ & 21,853 & 0.84 & 1.46 & 11,747 \\
\hline 10 & 2010 & Doc (3wk) & 21,930 & 1.08 & 1.83 & 21,270 \\
\hline 11 & 2013 & Nab-pac (3x weekly) & 15,229 & 0.81 & N.R. & 16,971 \\
\hline 11 & 2013 & Pac (3x weekly) & 12,609 & 0.64 & N.R. & 12,991 \\
\hline 12 & 2009 & Lap+cap (14,3wk) & 59,133 & N.R. & 1.45 & - \\
\hline 12 & 2009 & Cap $(14,3 w k)$ & 41,677 & N.R. & 1.29 & - \\
\hline 13 & 2001 & Pac (3wk) & 11,507 & 0.35 & 0.76 & 2,493 \\
\hline 13 & 2001 & Doc (3wk) & 18,421 & 0.34 & 0.76 & $-4,821$ \\
\hline 13 & 2001 & $\operatorname{Vino}(1,8,3 w k)+M(3 w k)$ & 8,016 & 0.43 & 0.85 & 9,184 \\
\hline 13 & 2001 & $M(6 w k)+V(3 w k)$ & 4,397 & 0.29 & 0.70 & 7,203 \\
\hline
\end{tabular}


Appendix 4.5: (continued)

\begin{tabular}{|c|c|c|c|c|c|c|}
\hline Studies & $\begin{array}{l}\text { Publication } \\
\text { year }\end{array}$ & Treatment & $\begin{array}{l}\text { Total } \\
\text { costs }^{a}\end{array}$ & $\begin{array}{l}\text { QALY } \\
\text { gained }\end{array}$ & LY gained & $\mathrm{NMB}^{\mathrm{a}}$ \\
\hline 14 & 2008 & No test, chemo alone & 33,502 & 1.28 & N.R. & 17,698 \\
\hline 14 & 2008 & $\mathrm{IHC}$ test, trast and chemo for $\mathrm{IHC} 3+$ & 39,939 & 1.41 & N.R. & 16,381 \\
\hline 14 & 2008 & $\begin{array}{l}\mathrm{IHC} \text { test, trast and chemo for IHC } \\
2+\text { and } 3+\end{array}$ & 44,286 & 1.46 & N.R. & 13,954 \\
\hline 14 & 2008 & $\begin{array}{l}\text { IHC test, FISH confirmation for } 2+ \\
\text { and } 3+\text {, trast and chemo for FISH+ }\end{array}$ & 42,094 & 1.46 & N.R. & 16,146 \\
\hline 14 & 2008 & $\begin{array}{l}\text { FISH test, trast and chemo for } \\
\mathrm{FISH}+\text { patients }\end{array}$ & 42,947 & 1.47 & N.R. & 15,893 \\
\hline 15 & 2013 & Eribulin (N.S.) & N.R. & N.R. & N.R. & - \\
\hline 15 & 2013 & TPC (N.S.) & N.R. & N.R. & N.R. & - \\
\hline 15 & 2013 & Cap (N.S.) & N.R. & N.R. & N.R. & - \\
\hline 15 & 2013 & Nap-pac (N.S.) & N.R. & N.R. & N.R. & - \\
\hline 15 & 2013 & Doxil (N.S.) & N.R. & N.R. & N.R. & - \\
\hline 15 & 2013 & Ixa (N.S.) & N.R. & N.R. & N.R. & - \\
\hline 16 & 2012 & Lap+cap $(14,3 w k)$ & 55,251 & 0.96 & 1.70 & $-16,931$ \\
\hline 16 & 2012 & Cap $(14,3 w k)$ & 23,877 & 0.77 & 1.41 & 6,883 \\
\hline 16 & 2012 & Trast $(3 w k)+\operatorname{cap}(14,3 w k)$ & 65,941 & 0.83 & 1.47 & $-32,861$ \\
\hline 17 & 2010 & Cap $(14,3 w k)$ & 23,239 & 1.17 & 2.06 & 23,561 \\
\hline 17 & 2010 & Trast (3wk)+cap $(14,3 w k)$ & 57,253 & 1.51 & 2.64 & 3,147 \\
\hline 18 & 2012 & $\operatorname{Pac}($ N.S.) & 1,983 & 0.86 & N.R. & 32,217 \\
\hline 18 & 2012 & Pac+bev (N.S.) & 86,174 & 1.02 & N.R. & $-45,374$ \\
\hline 19 & 2005 & Trast (1wk) & 52,277 & N.R. & $0.3-0.7$ & - \\
\hline 20 & 2009 & Cap $(14,3 w k)$ & 25,559 & 0.54 & 0.84 & $-4,159$ \\
\hline 20 & 2009 & Ixa+cap $(14,3 w k)$ & 51,885 & 0.62 & 1.01 & $-26,965$ \\
\hline 21 & 2014 & Bev+chemo & N.R. & N.R. & N.R. & - \\
\hline 21 & 2014 & Taxane based chemotherapy & N.R. & N.R. & N.R. & - \\
\hline 22 & 2007 & $\operatorname{Pac}(3 w k)$ & 23,968 & 0.83 & 1.69 & 9,232 \\
\hline 22 & 2007 & $\operatorname{Gem}(1,8,3 w k)+\operatorname{pac}(3 w k)$ & 37,711 & 1.00 & 2.01 & 2,289 \\
\hline 23 & 2003 & Doc (3wk) & 10,059 & N.R. & N.R. & - \\
\hline 23 & 2003 & Cap $(14,3 w k)+$ doc $(3 w k)$ & 10,706 & N.R. & N.R. & - \\
\hline 24 & 2005 & Doc (3wk) & 23,258 & 0.66 & 1.16 & 3,142 \\
\hline 24 & 2005 & Cap $(14,3 w k)+$ doc (3wk) & 25,325 & 0.81 & 1.38 & 7,075 \\
\hline
\end{tabular}

${ }^{a}$ in $€ 2013 ;{ }^{b}$ health care perspective; ${ }^{c}$ societal perspective.

Legend: ana, anastrozole; bev, bevacizumab; cap, capecitabine; chemo, chemotherapy; trast, trastuzumab; doc, docetaxel; doxil, liposomal doxorubicin; FISH, fluorescent in situ hybridization; gem, gemcintabine; HR, hazard ratio; HT+, HercepTest positive; IHC, immunohistochemistry; ixa, ixapebilone; lap, lapatinib; let, letrozole; M, mitomicyn; nab-pac, albumin-bound paclitaxel; N.R., Not reported; N.S., administration schedule not specified; pac, paclitaxel; PD, progressed disease; PFS, progression-free survival; $V$, vinblastine; vino, vinorelbine; $1 w k$, weekly administration; $3 w k$, administration once each 3 weeks; $6 w k$, administration once each 6 weeks; 3x/week, 3times weekly; 1,8,3wk, administration on days 1,8, of 3weeks cycle; 3 out of 4, administration on days 1,8,15 of 4weeks cycle; 14,3wk, daily during 14days every 3weeks; 1 and 15, administration on day 1 and 15 of 4 weeks cycle. 
Appendix 4.6: Details about elicitation method of utilities and population in which utilities were elicitated.

\begin{tabular}{|c|c|c|}
\hline $\begin{array}{l}\text { Studies/ } \\
\text { Characteristics }\end{array}$ & Population where it was elicited & Instrument used for elicitation \\
\hline 1 & N.R. & N.R. \\
\hline 2 & N.R. & N.R. \\
\hline 3 & N.R. & N.R. \\
\hline 4 & Oncology nurses and nurses & SG \\
\hline 5 & Canadian population & TTO \\
\hline 6 & $\begin{array}{l}\text { MBC patients, general pop (for the } \\
\text { vignette based study) }\end{array}$ & FACT-G \& FACT-B, vignette based study \\
\hline 7 & $\begin{array}{l}\text { MBC patients, general pop (for the } \\
\text { vignette based study) }\end{array}$ & FACT-G \& FACT-B, vignette based study \\
\hline 8 & MBC patient (for pre-progression) & EQ-5D, utility decrement based on literature \\
\hline 9 & US oncology nurses & N.R. \\
\hline 10 & N.R. & N.R. \\
\hline 11 & N.R. & N.R. (pooled analysis of MBC utility weights) \\
\hline 12 & N.R. & N.R. \\
\hline 13 & N.R. & N.R. \\
\hline 14 & Swedish breast cancer patients & $E Q-5 D$ \\
\hline 15 & N.R. & N.R. \\
\hline 16 & $\mathrm{MBC}$ & EQ-5D (pre-progression), decrements \\
\hline 17 & N.R. & N.R. \\
\hline 18 & N.R. & N.R. \\
\hline 19 & N.A. & N.A. \\
\hline 20 & Patients & Health Utilities Index Mark 3 (HUI) \\
\hline 21 & N.R. & N.R. \\
\hline 22 & Oncology nurses anc clinical specialist & $\begin{array}{l}\text { SG (study which is used is a pooled estimate from } \\
\text { three studies using this methodology) }\end{array}$ \\
\hline 23 & N.A. & N.A. \\
\hline 24 & Nurses & SG (average of 3 studies) \\
\hline
\end{tabular}

Legend: EQ-5D, EuroQol - 5 dimension; FACT-B, Functional Assessment of Cancer Therapy - Breast; FACT-G, Functional Assessment of Cancer Therapy - General; MBC, metastatic breast cancer; N.A., not applicable; N.R., not reported; SG, standard gamble; TTO, time trade off. 
Appendix 4.7: Utilities used in each study

\begin{tabular}{|c|c|c|c|c|c|}
\hline Studies & $\begin{array}{l}\text { Health states } \\
\text { Response }\end{array}$ & Pre-progression & Progression & Terminal disease & $\begin{array}{l}\text { Utility decrements for } \\
\text { AEs }\end{array}$ \\
\hline 1 & 0.810 & 0.650 & 0.450 & & $0.11-0.21^{\mathrm{a}}$ \\
\hline 2 & & 0.740 & 0.440 & & \\
\hline 3 & 0.790 & 0.720 & 0.450 & & $0.06-0.07^{*}$ \\
\hline 4 & 0.840 & 0.620 & 0.330 & 0.130 & $b$ \\
\hline 5 & & 0.610 & 0.260 & & \\
\hline 6 & & 0.860 & 0.620 & & $0.03-0.15^{\mathrm{a}}$ \\
\hline 7 & & 0.860 & 0.620 & & N.R. \\
\hline 8 & & 0.694 & 0.472 & & \\
\hline 9 & 0.840 & 0.700 & 0.490 & & \\
\hline 10 & 0.790 & 0.720 & 0.450 & & $0.06-0.07^{g}$ \\
\hline 11 & 0.810 & 0.650 & 0.450 & & $0.11-0.21^{c}$ \\
\hline 12 & 0.840 & 0.700 & 0.500 & & \\
\hline 13 & N.R. & N.R. & N.R. & & \\
\hline 14 & N.R. & $0.685^{d}$ & N.R. & & \\
\hline 15 & 0.790 & $0.715^{\mathrm{e}}$ & 0.443 & 0.190 & $0.103-0.155^{\mathrm{a}}$ \\
\hline 16 & & 0.694 & 0.472 & & \\
\hline 17 & & 0.700 & 0.500 & & \\
\hline 18 & 0.790 & 0.715 & 0.443 & & $0.103-0.155^{\mathrm{a}}$ \\
\hline 19 & N.A. & N.A. & N.A. & & N.A. \\
\hline 20 & 0.670 & 0.650 & 0.610 & & \\
\hline 20 & 0.770 & 0.620 & 0.680 & & \\
\hline 21 & 1.000 & & 0.500 & & $0.250^{\mathrm{b}}$ \\
\hline 22 & $0.810\left(0.670^{f}\right)$ & $0.650\left(0.540^{f}\right)$ & $0.450\left(0.450^{f}\right)$ & & \\
\hline 23 & N.A. & N.A. & N.A. & & \\
\hline 24 & N.A. & 0.720 & 0.480 & & \\
\hline Minimum & 0.670 & 0.610 & 0.260 & 0.130 & $0.03-0.250$ \\
\hline Maximum & 1.000 & 0.860 & 0.680 & 0.190 & \\
\hline
\end{tabular}

${ }^{a}$ each $A E$ has a different utility decrement; ${ }^{b}$ each $A E$ is valued separately. Each has its own utility value assigned; ${ }^{c}$ AE and state-specific; ${ }^{d}$ called 'Metastatic breast cancer' in paper; ${ }^{e}$ called 'Baseline' in paper; ${ }^{f}$ with toxicities; ${ }^{g}$ treatment-specific.

Legend: N.A., not applicable; N.R., not reported. 


\section{References}

1. Alba $E$, Ciruelos E, Lopez R, et al. Cost-utility analysis of nanoparticle albumin-bound paclitaxel versus paclitaxel in monotherapy in pretreated metastatic breast cancer in Spain. Expert Rev Oharmacoecon Outcomes Res. 2013;13(3):381-391.

2. Athanasakis K, J K. A cost-effectiveness analysis of trastuzumab plus docetaxel vs. docetaxel alone for the treatment of HER2-positive metastatic breast cancer in the Greek healthcare setting. Forum of Clinical Oncology. 2012;2012(3):4.

3. Benedict A, Cameron DA, Corson $\mathrm{H}$, et al. An economic evaluation of docetaxel and paclitaxel regimens in metastatic breast cancer in the UK. PharmacoEconomics. 2009;27(10):847-859.

4. Brown RE, Hutton J, Burrell A. Cost effectiveness of treatment options in advanced breast cancer in the UK. PharmacoEconomics. 2001;19(11):1091-102. PubMed PMID: 11735676; eng.

5. Dedes KJ, Matter-Walstra K, Schwenkglenks M, et al. Bevacizumab in combination with paclitaxel for HER-2 negative metastatic breast cancer: an economic evaluation. Eur J Cancer. 2009;45(8): 1397-1406.

6. Delea T, Amdahl J, Chit A, et al. Cost-effectiveness of lapatinib plus letrozole in her2-positive, hormone receptor-positive metastatic breast cancer in Canada. Curr Oncol. 2013;20(5):e371.

7. Delea TE, Hawkes C, Amonkar MM, et al. Cost-Effectiveness of Lapatinib plus Letrozole in PostMenopausal Women with Hormone Receptor-and HER2-Positive Metastatic Breast Cancer. Breast Care. 2013;8(6):429-437.

8. Delea TE, Tappenden P, Sofrygin $\mathrm{O}$, et al. Cost-effectiveness of lapatinib plus capecitabine in women with HER2 + metastatic breast cancer who have received prior therapy with trastuzumab. Eur J Health Econ. 2012;13(5):589-603.

9. Elkin EB, Weinstein MC, Winer EP, et al. HER-2 testing and trastuzumab therapy for metastatic breast cancer: a cost-effectiveness analysis. J Clin Oncol. 2004;22(5):854-863.

10. Frias C, Cortes J, Segui MA, et al. Cost-effectiveness analyses of docetaxel versus paclitaxel once weekly in patients with metastatic breast cancer in progression following anthracycline chemotherapy, in Spain. Clin Transl Oncol. 2010;12(10):692-700.

11. Lazzaro C, Bordonaro R, Cognetti F, et al. An Italian cost-effectiveness analysis of paclitaxel albumin (nab-paclitaxel) versus conventional paclitaxel for metastatic breast cancer patients: the COSTANza study. ClinicoEconomics and outcomes research: CEOR. 2013;5:125.

12. Le QA, Hay JW. Cost-effectiveness analysis of lapatinib in HER-2-positive advanced breast cancer. Cancer. 2009;115(3):489-498.

13. Li $\mathrm{N}$, van Agthoven $\mathrm{M}$, Willemse $\mathrm{P}$, et al. A cost--utility analysis comparing second-line chemotherapy schemes in patients with metastatic breast cancer. Anti-Cancer Drugs. 2001;12(6):533-40.

14. Lidgren $\mathrm{M}$, Wilking $\mathrm{N}$, Jonsson $\mathrm{B}$, et al. Cost-effectiveness of HER2 testing and trastuzumab therapy for metastatic breast cancer. Acta Oncol. 2008;47(6):1018-1028.

15. Lopes G, Gluck S, Avancha K, et al. A cost effectiveness study of eribulin versus standard single-agent cytotoxic chemotherapy for women with previously treated metastatic breast cancer. Breast Cancer Res Treat. 2013;137(1):187-193.

16. Machado M, Einarson TR. Lapatinib in patients with metastatic breast cancer following initial treatment with trastuzumab: an economic analysis from the Brazilian public health care perspective. Breast Cancer. 2012;4:173-182.

17. Matter-Walstra KW, Dedes KJ, Schwenkglenks M, et al. Trastuzumab beyond progression: a costutility analysis. Ann Oncol. 2010;21(11):2161-2168.

18. Montero AJ, Avancha K, Gluck S, et al. A cost-benefit analysis of bevacizumab in combination with paclitaxel in the first-line treatment of patients with metastatic breast cancer. Breast Cancer Res Treat. 2012;132(2):747-751.

19. Norum J, Risberg T, Olsen JA. A monoclonal antibody against HER-2 (trastuzumab) for metastatic breast cancer: a model-based cost-effectiveness analysis. Ann Oncol. 2005;16(6):909-914. 
20. Reed SD, Li Y, Anstrom KJ, et al. Cost effectiveness of ixabepilone plus capecitabine for metastatic breast cancer progressing after anthracycline and taxane treatment. J Clin Oncol. 2009;27(13): 2185-2191.

21. Refaat T, Choi M, Gaber G, et al. Markov Model and Cost-Effectiveness Analysis of Bevacizumab in HER2-Negative Metastatic Breast Cancer. Am J Clin Oncol. 2014;37(5):480-485.

22. Takeda AL, Jones J, Loveman E, et al. The clinical effectiveness and cost-effectiveness of gemcitabine for metastatic breast cancer: a systematic review and economic evaluation. Health Technol Assess. 2007;11(19):iii, ix-xi, 1-62.

23. Verma S, llersich AL. Population-based pharmacoeconomic model for adopting capecitabine/ docetaxel combination treatment for anthracycline-pretreated metastatic breast cancer. Oncologist. 2003;8(3):232-240.

24. Verma S, Maraninchi D, O'Shaughnessy J, et al. Capecitabine plus docetaxel combination therapy. Cancer. 2005;103(12):2455-2465.

25. Miller K, Wang M, Gralow J, et al. Paclitaxel plus bevacizumab versus paclitaxel alone for metastatic breast cancer. N Engl J Med. 2007;357(26):2666-76. 


\section{Chapter 5}

\section{The relative effectiveness of eribulin for advanced breast cancer treatment: A study of the Southeast Netherlands advanced breast cancer registry}

Xavier G.L.V. Pouwels, Sandra M.E. Geurts, Bram L.T. Ramaekers, Frans Erdkamp, Birgit E.P.J. Vriens, Kirsten N.A. Aaldering, Yes A.J. van de Wouw, M.W. Dercksen, Tineke J. Smilde, Natasha A.J.B. Peters, Anne-Marie van Riel, Manon .J. Pepels, José Heijnen-Mommers, Manuela A. Joore, Vivianne C.G. Tjan-Heijnen, Maaike de Boer 


\section{Abstract}

\section{Background}

Eribulin provided significant overall survival (OS) benefit in heavily pre-treated advanced breast cancer patients in the EMBRACE trial. We investigated the use of eribulin in daily clinical practice, the relative effectiveness of eribulin versus non-eribulin chemotherapy, and the safety of eribulin in real world patients included in the SOutheast Netherlands Advanced BREast cancer (SONABRE) registry.

\section{Materials and Methods}

Patients treated with eribulin and eligible patients for eribulin who received a different chemotherapy (i.e. non-eribulin group) in ten hospitals in 2013-2017 were included. A multivariate matching algorithm was applied to correct for differences in baseline characteristics between the groups, including the number of previous treatment lines. Progression-free survival (PFS) and OS of eribulin were compared with the matched noneribulin group through Kaplan-Meier curves and multivariate Cox proportional hazard models. The occurrence of dose delay and reduction was described.

\section{Results}

Forty-five patients received eribulin according to its registration criteria and 74 patients were eligible for eribulin but received non-eribulin chemotherapy. Matching increased the similarity in baseline characteristics between the eribulin and non-eribulin groups. Median PFS was 3.5 months (95\% confidence interval $(\mathrm{Cl}) 2.7-5.5)$ in the eribulin group and 3.2 months $(95 \% \mathrm{Cl}$ 2.0-4.8) in the matched non-eribulin group (adjusted hazard ratio (HR): $0.83,95 \% \mathrm{Cl} 0.49-1.38$ ). Median OS was 5.9 months $(95 \% \mathrm{Cl} 4.6-11.0)$ and 5.2 months $(95 \% \mathrm{Cl} 4.6-9.5)$ in the eribulin and non-eribulin groups, respectively (adjusted HR: $0.66,95 \% \mathrm{Cl} 0.38-1.13$ ). Dose delay or reduction occurred in 14 patients (31\%) receiving eribulin.

\section{Conclusions}

No difference in PFS and OS was observed between eribulin and non-eribulin treated patients. Eribulin had a manageable toxicity profile. 


\section{Introduction}

Eribulin mesylate (eribulin) is a non-taxane inhibitor of microtubule dynamics of the halichondrin class of anti-neoplastic drugs. ${ }^{1}$ In 2011, eribulin provided statistically significant overall survival (OS) benefits of 2.5 months in heavily pre-treated advanced breast cancer $(A B C)$ patients compared with patients receiving treatment of physician's choice in the phase III EMBRACE trial (hazard ratio (HR) for OS: $0.81,95 \%$ confidence interval (CI) 0.66-0.99). ${ }^{2}$ Eribulin was approved for $A B C$ treatment in March 2011 and reimbursed in July 2013 in the Netherlands.

Investigating the use and relative effectiveness of a drug in daily clinical practice is important in the $A B C$ context because only a small percentage of $A B C$ patients participate in clinical trials ${ }^{3}$, and patients included in clinical trials are not representative of patients treated in clinical practice. ${ }^{4}$ Therefore, clinical practice data on the use, relative effectiveness, and safety of a new drug for $A B C$ treatment are essential as an addition to clinical trial data. ${ }^{5}$ Previous non-comparative real-world and expanded access studies reached similar progression-free survival (PFS) and OS estimates with eribulin treatment as in EMBRACE, and confirmed the tolerable toxicity profile of this treatment. ${ }^{6-10}$ These studies did not provide data whether eribulin was administered according to its indication criteria.

To the best of our knowledge, only three studies investigated the relative effectiveness of eribulin in $A B C$ patients using non-randomized evidence. ${ }^{11-13}$ The first study found a statistically significant improvement in OS in Japanese patients treated with eribulin versus patients treated with taxane monotherapy or taxane chemotherapy with bevacizumab. ${ }^{12}$ The second study did not observe an improved time to treatment failure when eribulin was compared with capecitabine, gemcitabine, and vinorelbine in metastatic triple negative (TN) breast cancer patients treated in US community oncology practices. ${ }^{11}$ The third study observed an improvement in PFS and OS in patients treated with eribulin compared to patients treated with 'other chemotherapy' as second, third or fourth line treatment in France. ${ }^{13}$

In the Netherlands, eribulin is indicated for $A B C$ patients who have received at least two chemotherapy regimens in the advanced setting. Prior treatments should include an anthracycline- and a taxane-based chemotherapy (during (neo)adjuvant or palliative treatment), if not contra-indicated. Since previous non-randomized comparisons did not apply these eligibility criteria ${ }^{11-13}$, the effectiveness of eribulin in this patient population remains unknown. Hence, a comparison of eribulin to noneribulin chemotherapies using non-randomized evidence and the stricter Dutch eligibility criteria is warranted to inform whether eribulin has an added value for treating $A B C$ in this setting. 
The aim of the current study was to investigate the use, the relative effectiveness and the safety of eribulin in the Dutch daily clinical practice, using data from the SOutheast Netherlands Advanced BREast cancer (SONABRE) registry.

\section{Methods}

\subsection{Study population}

Data were obtained from the SONABRE registry (NCT03577197), which included patients diagnosed with $A B C$ from 2007 onwards in 12 hospitals in the Southeast of the Netherlands. This registry contains patient and treatment information (patient and tumor characteristics, treatment, toxicity, hospitalizations, and outcomes, i.e. progression and death) collected retrospectively from medical files by trained registration clerks. The Medical Research Ethics Committee of the Maastricht University Medical Centre+ approved the initiation of the registry (No. 15-4-239). For the current study, we identified all patients who received eribulin, and patients who were eligible for eribulin after July $1^{\text {st }}, 2013$ (registration date of eribulin in The Netherlands), but received a different chemotherapy than eribulin (non-eribulin group). Eligible patients had received at least two prior chemotherapy in the advanced setting, a prior anthracycle- and a prior taxane-based chemotherapy (in any setting), if not contra-indicated. The data lock for the current analysis was October $23^{\text {rd }}, 2017$.

\subsection{Endpoints definition}

The endpoints of this study were the use of eribulin in daily clinical practice, the relative effectiveness of eribulin versus non-eribulin chemotherapies, and the safety of eribulin treatment.

The use of eribulin in daily clinical practice was described as the proportion of patients who received eribulin in the group of patients eligible for eribulin treatment, and the number of patients who received eribulin according to its indication criteria or not. Additionally, the treatment line in which patients received eribulin, the number of administered eribulin cycles, and the starting dose of eribulin treatment were considered.

The relative effectiveness of eribulin versus non-eribulin chemotherapy was investigated in terms of PFS and OS. PFS was defined as the time between the start of treatment under study and the occurrence of disease progression or death. Progression was reported by the treating physician using radiographic imaging, tumor markers, and/or symptoms of the patient. Patients were censored for PFS at the start 
of a subsequent treatment when progression was not registered (before the initiation of this subsequent treatment) or at their last follow-up date when they were still alive without having experienced disease progression yet. OS was defined as the time between the start of treatment and death. Patients alive at the end of follow-up were censored for OS.

The safety of eribulin was defined based on two outcomes. The first was the occurrence of specific toxicities (e.g. neutropenia, febrile neutropenia) causing dose delay and/or reduction. The second was "any toxicity causing hospitalization", of which the type of toxicity is not specified in the registry.

\subsection{Statistical analyses}

This analysis compared eribulin to a treatment line matched non-eribulin group, using a multivariate matching algorithm [genetic matching (GenMatch)], to correct for differences in baseline characteristics between the groups. ${ }^{14,15}$ Treatment line matching means that each treatment line administered to eligible comparator patients was separately included in the eligible comparator group. This approach to matching (i.e. treatment line matching) differs from the classical way of matching (i.e. patient level matching) because different 'versions' of each comparator patient are included in the eligible comparator group instead of considering patients for matching only when they become eligible for the intervention.

GenMatch was performed according to the following steps, using the "Matching" package in the statistical software . $^{15}$ First, baseline characteristics of all groups were examined to investigate whether baseline characteristics overlapped because matching methods are not recommended in case of poor overlap in baseline characteristics ("ggplot" density plots in R). Second, covariates that were expected to influence treatment assignment and prognosis were selected based on clinical expert opinion. These covariates were the age at the start of treatment, the tumor subtype [based on the hormonal (HR) status (positive, negative, unknown) and the human epidermal growth factor receptor 2 (HER2) status of the tumor (positive, negative, unknown)], the number of previous chemotherapy treatments received in the advanced setting $(2,3$, and $\geq 4)$, and the presence of central nervous system (CNS) metastasis. Third, a propensity score for treatment assignment including these covariates was calculated based on a logistic regression ("glm" function). Fourth, the propensity score and these covariates were included in the GenMatch algorithm. Matching 1:1, with replacement was performed ("GenMatch" and "Match" functions). ${ }^{14}$ Fifth, covariates balance was assessed using two methods. Covariates balance of all covariates included in the GenMatch algorithm was investigated 
through visual inspection of quantile-quantile plots ( $Q Q$ plots) and the variance ratio (ratio of the variance of the covariate values of the eribulin group and the variance of the covariate values of the non-eribulin groups) ("MatchBalance" function). Additionally, covariate balance of continuous and ordinal variables with more than one category (age at treatment initiation, tumor subtypes, number of previous chemotherapy received) was assessed through the Kolmorogov-Smirnov bootstrap $p$ values ("MatchBalance" function), while the covariate balance of variable with dichotomous values (presence of CNS metastasis) was assessed by comparing the proportion of patients having CNS in each group.

All results presented for the eribulin group include only patients who received eribulin according to its indication criteria. Descriptive statistics were used to summarize patient and tumor characteristics and the occurrence of toxicities. KaplanMeier curves were estimated for PFS and OS for the eribulin and treatment line matched non-eribulin group. Median PFS and OS (and 95\% confidence interval (CI)) were retrieved from the Kaplan-Meier curves. Multivariate Cox proportional hazard (PH) models (adjusted for the covariates included in the GenMatch algorithm) were fitted to estimate the relative effectiveness of eribulin versus non-eribulin chemotherapy on PFS and OS. The base-case analysis compared eribulin to the treatment line-matched non-eribulin group. In addition, two sensitivity analyses were performed. These analyses compared eribulin with a patient level matched noneribulin group (the same matching algorithm was used for this comparison) and eribulin with an unmatched non-eribulin group (no matching performed). All statistical analyses were performed using the statistical software R. ${ }^{16}$ The statistical significance level was $5 \%$.

\section{Results}

\subsection{Study Population}

At the database lock, 119 patients from ten hospitals were eligible for eribulin after July $1^{\text {st }}, 2013$. A total of $38 \%(45 / 119)$ of these patients received eribulin according to its registration criteria, while $62 \%$ (74/119) were treated with non-eribulin chemotherapy. An additional 15 patients (25\% of the 60 patients who received eribulin) did not receive eribulin according to its registration criteria because those patients did not receive two previous chemotherapy lines in the advanced setting $(\mathrm{N}=5)$, did not receive an anthracycline-based chemotherapy $(\mathrm{N}=11)$, did not receive a 
taxane-based chemotherapy $(\mathrm{N}=5)$, and/or did receive concomitant trastuzumab $(\mathrm{N}=4)$ (multiple reasons may apply to a single patient).

Among the 45 patients who received eribulin according to its registration criteria, half received eribulin as fourth or later chemotherapy line for $A B C$ (median previous number of chemotherapy line of 3 (range: 2-7)), and 26 patients (58\%) received eribulin as last treatment. The starting dose of eribulin of $2(4 \%)$ patients was missing, all other patients received the full eribulin dose at treatment initiation $\left(1,23 \mathrm{mg} / \mathrm{m}^{2}\right)$. The median number of administered cycles was 4 (range: 1-27).

The $74(62 \%)$ patients eligible for eribulin treatment who did not receive eribulin (i.e. unmatched non-eribulin group) received a total of 102 chemotherapy lines after becoming eligible for eribulin (i.e. considered in treatment line matching).

The median age at the start of treatment was 61 years (range: $41-73$ ) in the eribulin group and 56 years (range: $40-74$ ) in the treatment line matched non-eribulin group. The proportion of patients with ER+/HER2+ (9\%) and ER+/HER2- (56\%) tumors was similar between the eribulin and the treatment line matched non-eribulin group. The proportion of patients with triple negative tumors in the eribulin group and treatment line matched non-eribulin group were $24 \%$ and $31 \%$ respectively. The proportion of patients with CNS metastasis in each group was $22 \%$ and $16 \%$, respectively. Thirty-seven percent of patients in both groups received 4 or more previous chemotherapy lines (Table 5.1). Thirteen different chemotherapy regimens were administered to patients in the treatment line matched non-eribulin group. Capecitabine $(N=11,24 \%)$, vinorelbine $(n=10,22 \%)$ and carboplatin $(n=6,13 \%)$ were administered the most often (Table 5.2).

Patient characteristics of the patient level matched non-eribulin group are provided in the Tables 2 \& 3 of the Appendix 5.1. Compared with the unmatched non-eribulin group, treatment line matching improved covariates balance of all covariates included in the matching algorithm, except the age at treatment initiation. Treatment line matching improved covariates balance of two covariates (tumor subtype and number of previous chemotherapy received) included in the matching algorithm compared with patient level matching, and it improved the variance ratio of all covariates included in the matching algorithm (Table 1 and Figures 3-6 of the Appendix5.1). 
Table 5.1: Patient characteristics in each group.

\begin{tabular}{|c|c|c|c|c|c|c|c|}
\hline \multicolumn{2}{|l|}{ Treatment group } & Eribulin (N) & $\%$ & $\begin{array}{l}\text { Treatment line } \\
\text { matched non- } \\
\text { eribulin }\end{array}$ & $\%$ & $\begin{array}{l}\text { Unmatched } \\
\text { non-eribulin }\end{array}$ & $\%$ \\
\hline \multicolumn{2}{|c|}{ Number of patients } & 45 & & 45 & & 74 & \\
\hline \multicolumn{2}{|c|}{ Age, median (min-max) } & $61(41-73)$ & & $56(40-74)$ & & $60(29-77)$ & \\
\hline \multirow[t]{5}{*}{ Tumor subtype } & $\mathrm{ER}+/ \mathrm{HER} 2-$ & 25 & $56 \%$ & 25 & $56 \%$ & 45 & $61 \%$ \\
\hline & $\mathrm{ER}+/ \mathrm{HER} 2+$ & 4 & $9 \%$ & 4 & $9 \%$ & 18 & $24 \%$ \\
\hline & ER-/HER2+ & 2 & $4 \%$ & 2 & $4 \%$ & 5 & $7 \%$ \\
\hline & Triple negative & 11 & $24 \%$ & 14 & $31 \%$ & 6 & $8 \%$ \\
\hline & Unknown & 3 & $7 \%$ & 0 & $0 \%$ & 0 & $0 \%$ \\
\hline \multirow{5}{*}{$\begin{array}{l}\text { Dominant } \\
\text { metastatic site }\end{array}$} & CNS & 10 & $22 \%$ & 7 & $16 \%$ & 12 & $16 \%$ \\
\hline & Visceral & 31 & $69 \%$ & 35 & $78 \%$ & 52 & $70 \%$ \\
\hline & Non-visceral & 4 & $9 \%$ & 3 & $7 \%$ & 10 & $14 \%$ \\
\hline & Bone only ${ }^{a}$ & 2 & $4 \%$ & 3 & $7 \%$ & 9 & $12 \%$ \\
\hline & Unknown & 0 & $0 \%$ & 0 & $0 \%$ & 0 & $0 \%$ \\
\hline \multirow{5}{*}{$\begin{array}{l}\text { Number of } \\
\text { metastatic sites }\end{array}$} & 1 & 2 & $4 \%$ & 4 & $9 \%$ & 12 & $16 \%$ \\
\hline & 2 & 12 & $27 \%$ & 11 & $24 \%$ & 17 & $23 \%$ \\
\hline & 3 & 15 & $33 \%$ & 10 & $22 \%$ & 18 & $24 \%$ \\
\hline & 4 & 8 & $18 \%$ & 13 & $29 \%$ & 14 & $19 \%$ \\
\hline & $5+$ & 8 & $17 \%$ & 7 & $16 \%$ & 13 & $17 \%$ \\
\hline \multirow{5}{*}{$\begin{array}{l}\text { Number of } \\
\text { previous } \\
\text { endocrine } \\
\text { therapy lines in } \\
\text { the } A B C \text { setting }\end{array}$} & 0 & 16 & $36 \%$ & 19 & $42 \%$ & 20 & $27 \%$ \\
\hline & 1 & 7 & $16 \%$ & 6 & $13 \%$ & 13 & $18 \%$ \\
\hline & 2 & 7 & $16 \%$ & 3 & $7 \%$ & 13 & $18 \%$ \\
\hline & 3 & 5 & $11 \%$ & 9 & $20 \%$ & 16 & $22 \%$ \\
\hline & $4+$ & 10 & $22 \%$ & 8 & $18 \%$ & 12 & $16 \%$ \\
\hline \multirow{3}{*}{$\begin{array}{l}\text { Number of } \\
\text { previous chemo- } \\
\text { therapy lines in } \\
\text { the } A B C \text { setting }\end{array}$} & 2 & 15 & $33 \%$ & 17 & $38 \%$ & 49 & $66 \%$ \\
\hline & 3 & 13 & $29 \%$ & 11 & $24 \%$ & 13 & $18 \%$ \\
\hline & $4+$ & 17 & $37 \%$ & 17 & $37 \%$ & 12 & $16 \%$ \\
\hline \multirow{4}{*}{$\begin{array}{l}\text { Number of } \\
\text { previous } \\
\text { chemotherapy } \\
\text { lines in all } \\
\text { settings, i.e. } \\
\text { (neo)adjuvant } \\
\text { and ABC settings }\end{array}$} & 2 & 4 & $9 \%$ & 3 & $7 \%$ & 9 & $12 \%$ \\
\hline & 3 & 13 & $29 \%$ & 17 & $38 \%$ & 41 & $55 \%$ \\
\hline & 4 & 13 & $29 \%$ & 14 & $31 \%$ & 16 & $22 \%$ \\
\hline & $5+$ & 15 & $33 \%$ & 11 & $24 \%$ & 8 & $11 \%$ \\
\hline
\end{tabular}

${ }^{a}$ Subgroup of non-visceral metastasis

Abbreviations: $A B C$, advanced breast cancer; CNS, central nervous system; ER, endocrine receptor status, HER2, human epidermal growth factor 2 receptor.

\subsection{Relative effectiveness of eribulin}

Median PFS was 3.5 months $(95 \% \mathrm{Cl} 2.7-5.5)$ in the eribulin group and 3.2 months $(95 \% \mathrm{Cl}$ 2.0-4.8) in the treatment line matched non-eribulin group (Figure 5.1A). This resulted in an adjusted $\mathrm{HR}$ for PFS of $0.83(95 \% \mathrm{Cl} 0.49-1.38)$ for eribulin versus treatment line matched non-eribulin chemotherapy (Table 5.3). Median OS was 5.9 months ( $95 \% \mathrm{Cl} 4.6-11.0$ ) in the eribulin group and 5.2 months $(95 \% \mathrm{Cl} 4.6-9.5)$ in the treatment line matched noneribulin group (Figure 5.1B). This resulted in an adjusted HR for OS of 0.66 (95\% Cl 0.38 1.13) for eribulin versus treatment line matched non-eribulin chemotherapy (Table 5.3). 
Table 5.2: Chemotherapy regimens administered to the treatment line matched non-eribulin group, $n=45$.

\begin{tabular}{lcc}
\hline Chemotherapy regimens & $\mathbf{N}$ & $\%$ \\
\hline Anthracycline-based chemotherapy & & $11 \%$ \\
$\quad$ Doxorubicin in non-pegylated liposomes & 5 & $4 \%$ \\
Taxane-based chemotherapy & 2 & $2 \%$ \\
Docetaxel & 1 & $2 \%$ \\
Paclitaxel & 1 & $24 \%$ \\
Paclitaxel, trastuzumab, carboplatin, pertuzumab & & $2 \%$ \\
Capecitabine-based chemotherapy & 11 & $2 \%$ \\
Capecitabine & 1 & $13 \%$ \\
Capecitabine-lapatinib & 1 & $2 \%$ \\
Capecitabine-Neratinib & & \\
Carboplatin-based chemotherapy & 6 & $7 \%$ \\
Carboplatin & 1 & $4 \%$ \\
Carboplatin-paclitaxel weekly & & $22 \%$ \\
Other & 3 & $2 \%$ \\
Gemcitabine & 2 & 10 \\
Vinorelbine & 1 & \\
CMF - trastuzumab & & \\
\hline
\end{tabular}

${ }^{a}$ (intravenous cyclophosphamide).

Abbreviations: CMF, cyclophosphamide, methotrexate and 5-fluorouracil; T-DM1, trastuzumab-emtansine.
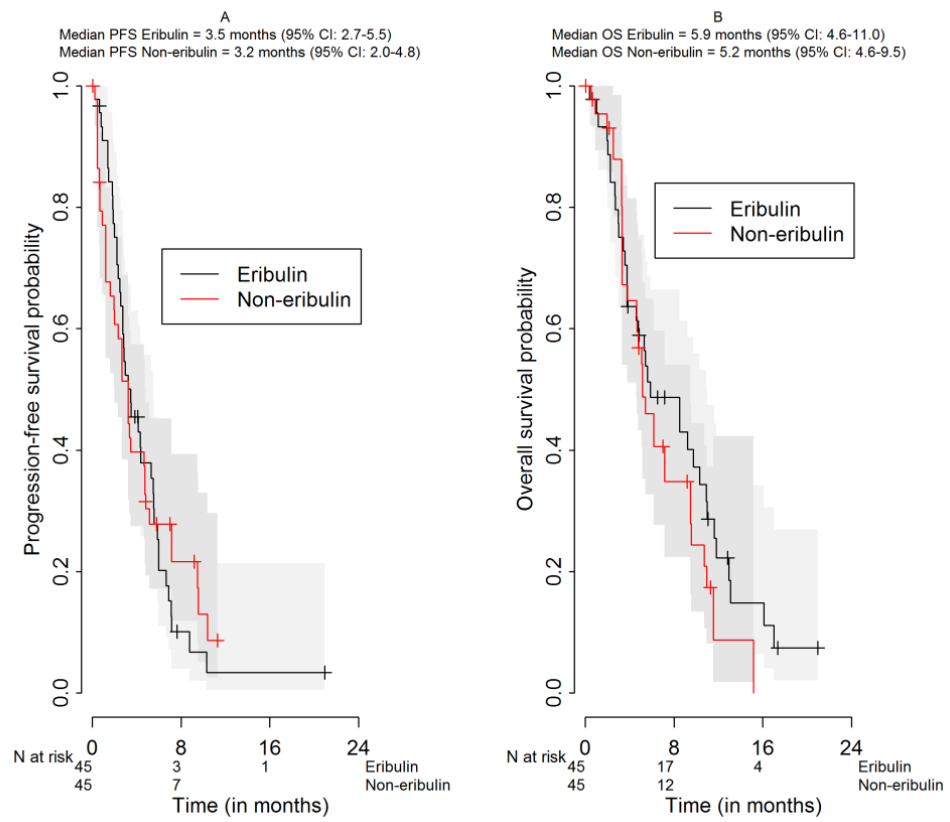

Figure 5.1: Comparison of progression-free survival (A) and overall survival (B) between the eribulin and the treatment line matched non-eribulin group. 


\subsubsection{Sensitivity analyses}

In the patient level matched non-eribulin group, median PFS was 2.7 months $(95 \% \mathrm{Cl}$ 1.8-4.7), and median OS was 6.8 months ( $95 \% \mathrm{Cl} 5.7-9.5)$. This resulted in an adjusted HR for PFS of 0.91 (95\% Cl 0.57-1.45), and an adjusted HR for OS of 0.62 ( $95 \% \mathrm{Cl} 0.37-$ 1.04) for eribulin versus patient level matched non-eribulin chemotherapy. Median PFS and OS in the unmatched non-eribulin group were 3.4 months $(95 \% \mathrm{Cl}$ 2.3-5.2) and 8.2 months (95\% $\mathrm{Cl} 5.9-11.0)$. This resulted in an adjusted HR for PFS of 0.99 (95\% $\mathrm{Cl} 0.60-1.62)$, and an adjusted $\mathrm{HR}$ for OS of 0.73 (95\% $\mathrm{Cl} 0.43-1.24)$ for eribulin versus unmatched non-eribulin chemotherapy. Results of these analyses are provided in the Appendix 5.1 (Table 4 and Figures 9-12).

Table 5.3: Results of the multivariate Cox proportional hazard models.

\begin{tabular}{|c|c|c|c|c|c|c|c|}
\hline \multirow[b]{2}{*}{ Covariates } & & \multicolumn{3}{|c|}{ PFS } & \multicolumn{3}{|c|}{ OS } \\
\hline & & $\begin{array}{l}\text { Coefficient } \\
\text { (SE) }\end{array}$ & $\begin{array}{c}\text { Hazard ratio } \\
(95 \% \mathrm{Cl})\end{array}$ & Significance & $\begin{array}{l}\text { Coefficient } \\
\text { (SE) }\end{array}$ & $\begin{array}{c}\text { Hazard ratio } \\
(95 \% \mathrm{Cl})\end{array}$ & Significance \\
\hline \multirow[t]{2}{*}{ Treatment } & $\begin{array}{l}\text { Non- } \\
\text { eribulin }\end{array}$ & Reference & 1.00 & - & - & 1.00 & - \\
\hline & Eribulin & $\begin{array}{l}-0.19 \\
(0.26)\end{array}$ & $\begin{array}{c}0.83 \\
(0.49-1.38)\end{array}$ & 0.47 & $\begin{array}{l}-0.42 \\
(0.27)\end{array}$ & $\begin{array}{c}0.66 \\
(0.38-1.13)\end{array}$ & 0.13 \\
\hline \multirow[t]{4}{*}{$\begin{array}{l}\text { Tumor } \\
\text { subtype }\end{array}$} & $\begin{array}{l}\mathrm{HR}+/ \mathrm{HER} 2- \\
\mathrm{HR}+/ \mathrm{HER} 2+\end{array}$ & $\begin{array}{c}\text { Reference } \\
-0.60 \\
(0.47)\end{array}$ & $\begin{array}{c}1.00 \\
0.55 \\
(0.22-1.37)\end{array}$ & 0.20 & $\begin{array}{c}- \\
-1.05 \\
(0.51)\end{array}$ & $\begin{array}{c}1.00 \\
0.35 \\
(0.13-0.94)\end{array}$ & $\begin{array}{c}- \\
0.04\end{array}$ \\
\hline & HR-/HER2+ & $\begin{array}{c}0.01 \\
(0.66)\end{array}$ & $\begin{array}{c}1.01(0.28- \\
3.66)\end{array}$ & 0.98 & $\begin{array}{l}-0.42 \\
(0.76)\end{array}$ & $\begin{array}{c}0.66 \\
(0.15-2.93)\end{array}$ & 0.58 \\
\hline & $\begin{array}{l}\text { Triple } \\
\text { negative }\end{array}$ & $\begin{array}{l}1.02 \\
(0.28)\end{array}$ & $\begin{array}{c}2.76 \\
(1.58-4.81)\end{array}$ & $<0.01$ & $\begin{array}{c}0.97 \\
(0.31)\end{array}$ & $\begin{array}{c}2.63 \\
(1.44-4.78)\end{array}$ & $<0.01$ \\
\hline & Unknown & $\begin{array}{c}0.41 \\
(0.72)\end{array}$ & $\begin{array}{c}1.50 \\
(0.37-6.12)\end{array}$ & 0.57 & $\begin{array}{c}0.95 \\
(0.74)\end{array}$ & $\begin{array}{c}2.60 \\
(0.61-11.09)\end{array}$ & 0.20 \\
\hline \multicolumn{2}{|c|}{ Age at treatment start } & $\begin{array}{l}<-0.01 \\
(0.01)\end{array}$ & $\begin{array}{c}0.99 \\
(0.96-1.02)\end{array}$ & 0.59 & $\begin{array}{l}<0.01 \\
(0.02)\end{array}$ & $\begin{array}{c}1.01 \\
(0.98-1.04)\end{array}$ & 0.67 \\
\hline $\begin{array}{l}\text { Number of } \\
\text { previous } \\
\text { chemothera }\end{array}$ & $\begin{array}{l}2 \\
3\end{array}$ & $\begin{array}{c}\text { Reference } \\
-0.65 \\
(0.35)\end{array}$ & $\begin{array}{c}1.00 \\
0.52 \\
(0.26-1.04)\end{array}$ & - & $\begin{array}{c}- \\
-0.25 \\
(0.36)\end{array}$ & $\begin{array}{c}1.00 \\
0.78 \\
(0.39-1.58)\end{array}$ & 0.49 \\
\hline $\begin{array}{l}\text { py lines for } \\
\text { ABC }\end{array}$ & $4+$ & $\begin{array}{c}0.33 \\
(0.31)\end{array}$ & $\begin{array}{c}1.40 \\
(0.77-2.55)\end{array}$ & 0.27 & $\begin{array}{c}0.54 \\
(0.31)\end{array}$ & $\begin{array}{c}1.72 \\
(0.93-3.19)\end{array}$ & 0.08 \\
\hline $\begin{array}{l}\text { Presence of } \\
\text { CNS }\end{array}$ & $\begin{array}{l}\text { No CNS } \\
\text { metastasis }\end{array}$ & Reference & 1.00 & - & - & 1.00 & - \\
\hline metastasis & $\begin{array}{l}\text { CNS } \\
\text { metastasis }\end{array}$ & $\begin{array}{c}0.76 \\
(0.36) \\
\end{array}$ & $\begin{array}{c}2.15 \\
(1.06-4.33) \\
\end{array}$ & 0.03 & $\begin{array}{c}0.98 \\
(0.40)\end{array}$ & $\begin{array}{c}2.67 \\
(1.22-5.86) \\
\end{array}$ & 0.01 \\
\hline
\end{tabular}

Abbreviations: $\mathrm{Cl}$, confidence interval; CNS, central nervous system; HER2, human epidermal growth factor 2 receptor; HR, hormonal status; $S E$, standard error.

\subsection{Safety of Eribulin}

Twenty-one different toxicities caused 35 dose delays or dose reductions in $31 \%$ $(\mathrm{N}=14)$ of patients treated with eribulin (the same toxicity could cause multiple dose 
delay or reduction in a single patient). Leucopenia or neutropenia occurred in six patients (13\%) and was the most common toxicity leading to dose delay or reduction (Table 5.4). Febrile neutropenia led to dose delay or reduction in one patient (2\%). Two patients had missing data concerning hospitalizations. Among the remaining 43 patients, eight (19\%) were hospitalized due to toxicities. Two of these patients (5\%) were also hospitalized due to tumor related symptoms.

Table 5.4: Overview of adverse events leading to dose delay or reduction in the eribulin group, $n=45$.

\begin{tabular}{lcc}
\hline Toxicities & Number of patients & $\%$ \\
\hline Leucopenia or neutropenia & 6 & $13 \%$ \\
Neuropathy & 4 & $9 \%$ \\
Gastro-intestinal complaints & 3 & $7 \%$ \\
Fatigue & 2 & $4 \%$ \\
Febrile neutropenia & 1 & $2 \%$ \\
Stomatitis & 1 & $2 \%$ \\
Asymptomatic decreased left ventricular ejection fraction & 1 & $2 \%$ \\
Thrombocytopenia & 1 & $2 \%$ \\
Liver test abnormalities & 1 & $2 \%$ \\
Other & 1 & $2 \%$ \\
\hline
\end{tabular}

\section{Discussion}

This study aimed to investigate the daily use, relative effectiveness and safety of eribulin for $A B C$ treatment, based on data collected in the real-world SONABRE registry. This registry contains patient and treatment information on patients diagnosed with $A B C$ from 2007 onwards in 12 hospitals from the Southeast of the Netherlands. In this study, eribulin was administered according to its registration criteria to $38 \%$ of the eligible patient population and a quarter of patients who received eribulin did not meet its registration criteria. Treatment with eribulin resulted in a median PFS and OS of 3.5 months $(95 \% \mathrm{Cl} 2.7-5.5)$ and 5.9 months (95\% $\mathrm{Cl}$ 4.6-11.0), respectively. PFS and OS of patients treated with eribulin were not different from PFS and OS of the treatment line matched non-eribulin group (adjusted HR for PFS: $0.83,95 \% \mathrm{Cl} 0.49-1.38$; adjusted HR for OS: $0.66,95 \% \mathrm{Cl} 0.38-1.13)$. The sensitivity analyses led to similar findings. Fourteen patients (31\%) experienced dose delays or reductions due to toxicities during eribulin treatment.

The median PFS of eribulin treatment observed in this real world study (3.5 months) was comparable with the median PFS reported in the EMBRACE trial (3.8 months) $)^{2}$ and previous observational studies (range: $2.9-3.8$ months). ${ }^{6,7,9,13,17,18}$ Concerning the relative effectiveness of eribulin on PFS, our HR for PFS (HR: $0.83,95 \%$ $\mathrm{Cl} 0.49-1.38$ ) was similar as in EMBRACE (HR: $0.87,95 \% \mathrm{Cl} 0 \cdot 71-1 \cdot 05)^{2}$, and as the 
range of HR estimated by Dranitsaris et al. ${ }^{11}$ who estimated the time to treatment failure of eribulin versus capecitabine, gemcitabine, and vinorelbine in metastatic TN breast cancer patients treated in US community oncology practices. The estimated HR for eribulin versus capecitabine, gemcitabine, and vinorelbine in that study were 1.15 (95\% Cl 0.75-1.76), 0.62 (95\% Cl 0.34-1.13), and 1.00 (95\% Cl 0.67-1.67), respectively. ${ }^{11}$ Except for a recent French study in which PFS in third or fourth line was improved with eribulin compared with other chemotherapy ${ }^{13}$, our results are in line with previous randomized and non-randomized studies by showing that eribulin does not improve PFS or time to treatment failure versus non-eribulin chemotherapy regimens.

However, eribulin led to a significant OS improvement in EMBRACE (HR: 0.81, 95\% $\mathrm{Cl} 0.66-0.99$ ), in the Japanese non-randomized study of Kikuchi et al. [HR versus taxane monotherapy: 0.32 ( $95 \% \mathrm{Cl} 0.19-0.53)$, HR versus taxane with bevacizumab: $0.39(95 \% \mathrm{Cl} 0.23-0.67)]^{12}$, and in the retrospective real world study of Jacot et al.. ${ }^{13}$ Although these previous studies demonstrated a significant OS benefit for the eribulin group, we could not confirm these results. The HR for OS of eribulin in the current study showed no differences in OS between eribulin and non-eribulin treated patients (HR: 0.66, 95\% Cl 0.38-1.13). Also, the median OS under eribulin treatment in the current study (5.9 months) was noticeably shorter than the median OS observed in EMBRACE (13.2 months), and previous observational studies (range: 5.9-22.3 months)..$^{6-10,12,17-23}$ The absolute lower OS in our study is most likely due to the older age of our cohort (median age of 61 years old versus 55 years old in EMBRACE), the presence of CNS metastases in $22 \%$ of patients (versus $0 \%$ in EMBRACE), and potentially due to the extensive number of previous chemotherapy regimens administered to our patients (33\% of patients received 5 or more previous chemotherapy versus $20 \%$ in EMBRACE). However, our results are in line with the pooled analysis of observational studies performed by Voutsadakis. ${ }^{24}$ He showed that eribulin treatment led to shorter PFS and OS estimates in observational studies compared with randomized trials, and explained these discrepancies by the less favorable patient characteristics in observational studies compared with randomized trials (more pre-treatment and lower performance scores in observational studies). ${ }^{24}$ The results of the current study were not compared with the results of the trial comparing eribulin to capecitabine because this trial included patient who received eribulin as first line $(0.2 \%)$ and second line $(26.5 \%)^{25}$, and because the registration criteria of eribulin in the Netherlands in 2013, at the time of the start of this study, were based on the EMBRACE trial.

The current study confirms the predictable and manageable toxicity profile of eribulin. Febrile neutropenia leading to dose reduction (which is according to the 
manufacturers guideline) or delay occurred in $2 \%$ of our patients, and occurred in $5 \%$ of patients in the EMBRACE trial. ${ }^{2}$ As in EMBRACE, leucopenia or neutropenia was the most frequent toxicity (leading to dose delay or reduction), but the incidence of leucopenia or neutropenia (13\%) resulting in dose delay or reduction was lower in the current study than in the eribulin group of EMBRACE (grade 3-4 leucopenia=14\%, grade 3-4 neutropenia=45\%). Incidences of other toxicities (e.g. neuropathy, febrile neutropenia, and fatigue) were comparable to previous observational analyses of eribulin. ${ }^{6,8,9}$ Hospitalizations due to toxicity occurred in $31 \%$ of patients in this study, partly combined with tumor related symptoms, which can be explained by the extensively pretreated, late stage of metastatic disease in our patients.

The limitations of the current study are its non-randomized character and the small number of included treatment lines. We could there adjust the comparison for observed confounders only. However, due to the limited number of non-eribulin treatment lines, the GenMatch algorithm was not able to reduce all differences in baseline characteristics (e.g. proportion of triple negative tumor subtype). The remaining discrepancies in observed confounders at baseline have been accounted for through the multivariate Cox regression model. A strength of the current study is the availability of detailed outcomes per treatment lines (PFS, OS, dose delay and reduction). This level of detail in the collected data made that treatment line matching was feasible. Other strengths are the inclusion of patients from different types of hospitals (teaching, non-teaching and university), which increases the external validity of the results and the use of treatment line matching. In $A B C$, many patients are treated with multiple treatment lines, which may influence treatment effectiveness. We accounted for this potential effect by applying GenMatch on treatment lines.

In the current study, the Kaplan-Meier curves of the eribulin and non-eribulin groups cross each other, which indicates a violation of the $\mathrm{PH}$ assumption. The HRs obtained from the Cox $\mathrm{PH}$ models therefore provide an estimation of the average relative effectiveness of eribulin over time. In the sensitivity analyses, the median OS estimates of the non-eribulin groups were longer than the median OS estimate of the eribulin group, but the HRs were consistently in favor of eribulin $(H R<1.00)$. These observations should not be interpreted as a discrepancy in results because the median OS obtained from the Kaplan-Meier curves are point estimates, while the HR is an estimation of the relative treatment effectiveness across the entire time horizon.

Excluding the 15 patients (25\%) who received eribulin outside its indication criteria ensured that the GenMatch algorithm was applied on groups with similar inclusion criteria since treatment lines administered to non-eribulin treated patients were included in the GenMatch algorithm when these patients became eligible for eribulin. Including the eribulin patients who received eribulin outside its indication 
criteria would have created dissimilarities in baseline characteristics (e.g. number of pre-treatment) that would not have been addressed by the GenMatch algorithm.

This study confirms that applying treatment line matching is a viable solution to increase the similarity of baseline characteristics between the groups when using observational data. This is especially useful when the number of eligible comparator patients is small because different "versions" of the same patients are included in the comparator group, which increases its size. Treatment line matching is thus an extension of matching with replacement. Other types of malignancy and other disease areas where treatments are administered in subsequent treatment lines could profit from applying treatment line matching.

In conclusion, the current real-world study shows that there is no difference in effectiveness between eribulin and non-eribulin chemotherapy and that eribulin had a manageable toxicity profile. Therefore, eribulin is a treatment option for $A B C$ patient 


\section{References}

1. Eisai Ltd. ANNEX I: Summary of product characteristics Halaven. p. 26.

2. Cortes J, O'Shaughnessy J, Loesch D, et al. Eribulin monotherapy versus treatment of physician's choice in patients with metastatic breast cancer (EMBRACE): a phase 3 open-label randomised study. Lancet. 2011;377(9769):914-923.

3. Cardoso F. Metastatic breast cancer patients: the forgotten heroes! Breast. 2009;18(5):271-272.

4. Mol L, Koopman M, van Gils CW, et al. Comparison of treatment outcome in metastatic colorectal cancer patients included in a clinical trial versus daily practice in The Netherlands. Acta Oncol. 2013; 52(5):950-955.

5. Fleurence RL NH, Jansen JP. The Critical Role Of Observational Evidence In Comparative Effectiveness Research. Health Affairs. 2010;29(10):1826-1833.

6. Aftimos $P$, Polastro L, Ameye L, et al. Results of the Belgian expanded access program of eribulin in the treatment of metastatic breast cancer closely mirror those of the pivotal phase III trial. Eur J Cancer. 2016;60:117-124.

7. Dell'Ova M, De Maio E, Guiu S, et al. Tumour biology, metastatic sites and taxanes sensitivity as determinants of eribulin mesylate efficacy in breast cancer: results from the ERIBEX retrospective, international, multicenter study. BMC Cancer. 2015;15:659.

8. Gamucci T, Michelotti A, Pizzuti L, et al. Eribulin mesylate in pretreated breast cancer patients: a multicenter retrospective observational study. J Cancer. 2014;5(5):320-327.

9. Garrone O, Montemurro F, Saggia C, et al. Eribulin in pretreated metastatic breast cancer patients: results of the TROTTER trial-a multicenter retrospective study of eribulin in real life. Springerplus. 2016;5:59.

10. Sabatier R, Dieras V, Pivot X, et al. Safety Results and Analysis of Eribulin Efficacy according to Previous Microtubules-Inhibitors Sensitivity in the French Prospective Expanded Access Program for Heavily Pre-treated Metastatic Breast Cancer. Cancer Res Treat. 2018;50(4):1226-1237.

11. Dranitsaris $\mathrm{G}$, Beegle $\mathrm{N}$, Kalberer $\mathrm{T}$, et al. A comparison of toxicity and health care resource use between eribulin, capecitabine, gemcitabine, and vinorelbine in patients with metastatic breast cancer treated in a community oncology setting. J Oncol Pharm Pract. 2015;21(3):170-177.

12. Kikuchi $Y$, Uchida $Y$, Shirakawa K, et al. A multicenter, observational study of metastatic breast cancer patients who were treated with eribulin mesylate or taxane-based regimens. Asia-Pac J Clin Oncol. 2018;14(5):e231-e237.

13. Jacot W, Heudel PE, Fraisse J, et al. Real-life activity of eribulin mesylate among metastatic breast cancer patients in the multicenter national observational ESME program. Int J Cancer. 2019;145(12):3359-3369.

14. Stuart EA. Matching methods for causal inference: A review and a look forward. Stat Sci. 2010; 25(1):1-21.

15. Sekhon JS, Grieve RD. A matching method for improving covariate balance in cost-effectiveness analyses. Health Econ. 2012;21(6):695-714.

16. R Core Team. R: A Language and Environment for Statistical Computing. Vienna, Austria: R Foundation for Statistical Computing; 2017.

17. Brems-Eskildsen AS, Kristoffersen KB, Linnet S, et al. Efficacy and toxicity of eribulin treatment in metastatic breast cancer patients. Acta Oncol. 2019;58(1):119-121.

18. Ates $\mathrm{O}$, Babacan $\mathrm{T}$, Kertmen $\mathrm{N}$, et al. Efficacy and safety of eribulin monotherapy in patients with heavily pretreated metastatic breast cancer. J BUON. 2016;21(2):375-81.

19. Kessler L, Falato C, Margolin S, et al. A retrospective safety and efficacy analysis of the first patients treated with eribulin for metastatic breast cancer in Stockholm, Sweden. Acta Oncol. 2015;54(4): 522-529.

20. Poletti P, Ghilardi V, Livraghi L, et al. Eribulin mesylate in heavily pretreated metastatic breast cancer patients: current practice in an Italian community hospital. Future Oncol. 2014;10(2):233-239.

21. Ramaswami R, O'Cathail SM, Brindley JH, et al. Activity of eribulin mesylate in heavily pretreated breast cancer granted access via the Cancer Drugs Fund. Future Oncol. 2014;10(3):363-376. 
22. Quaquarini E, Sottotetti F, D'Ambrosio D, et al. Eribulin across multiple lines of chemotherapy: a retrospective study on quality of life and efficacy in metastatic breast cancer patients. Future Oncol. 2017;13(11s):11-23.

23. Barni S, Livraghi L, Morritti M, et al. Eribulin in the treatment of advanced breast cancer: real-world scenario from 39 Italian centers - ESEMPiO study. Future Oncol. 2019;15(1):33-44.

24. Voutsadakis IA. A systematic review and pooled analysis of retrospective series of eribulin in metastatic breast cancer. Anticancer Drugs. 2017;28(5):557-564.

25. Kaufman PA, Awada A, Twelves C, et al. Phase III open-label randomized study of eribulin mesylate versus capecitabine in patients with locally advanced or metastatic breast cancer previously treated with an anthracycline and a taxane. J Clin Oncol. 2015;33(6):594-601. 


\section{Appendices}

\section{Overlap in patient characteristics before matching}

In the following two figures, one can observe how baseline characteristics of the eribulin and unmatched non-eribulin (patient level and treatment lines separately) overlap before matching has been performed. For matching to be successful, baseline characteristics have to overlap, otherwise matching will not resolve the disbalance in baseline characteristics. In Figures S5.1 and S5.2, one can see that baseline characteristics overlap between eribulin en both non-eribulin groups, hence, matching is a suitable method to adjust the comparison of eribulin versus non-eribulin.
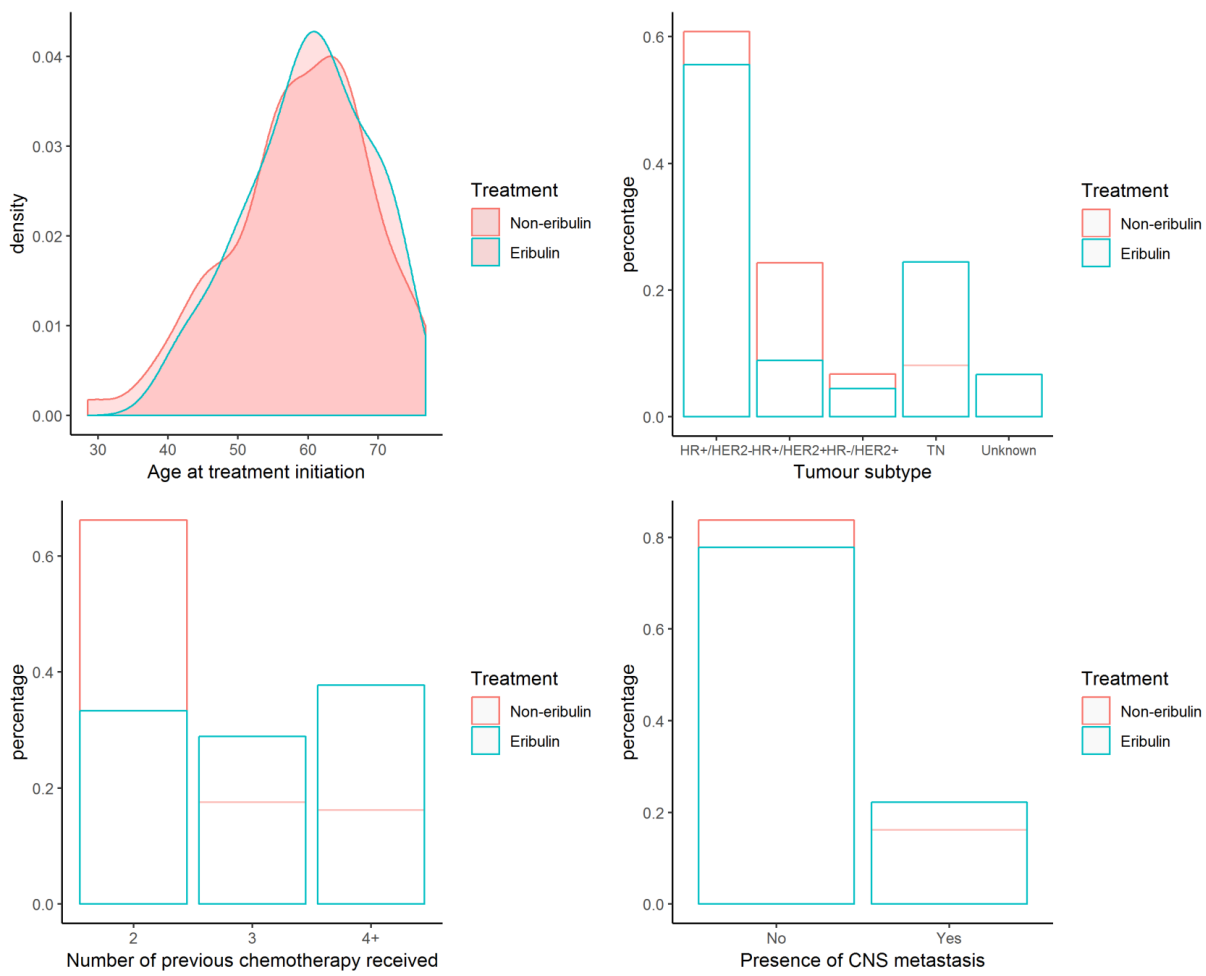

Figure S5.1: Overlap in baseline characteristics between the eribulin group and the unmatched noneribulin group (patient level). 

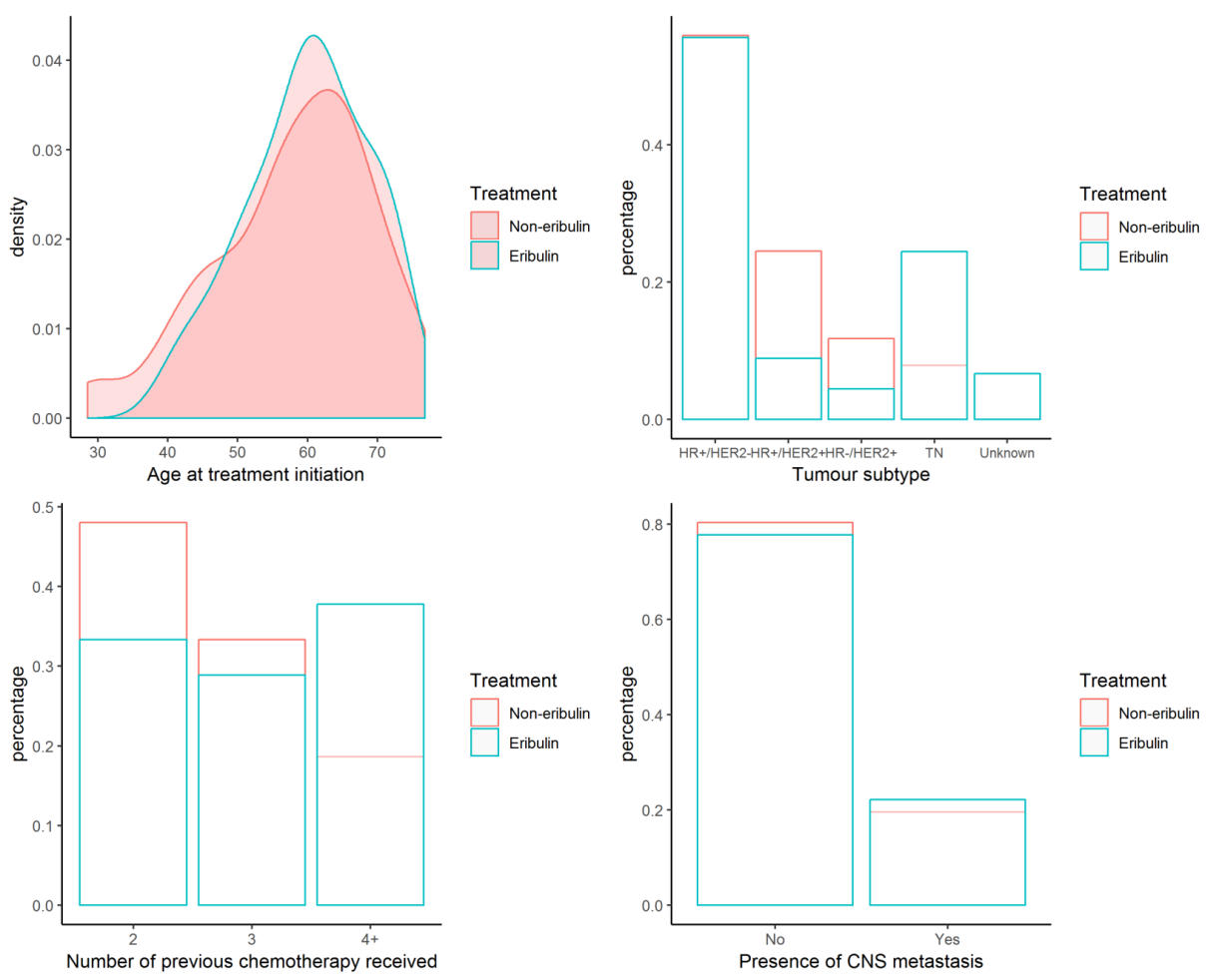

Figure S5.2: Overlap in baseline characteristics between the eribulin group and the unmatched noneribulin treatment lines.

\section{Covariate balance comparison between the different non- eribulin groups}

Covariates balance was improved for tumour subtype, and the number of previous chemotherapy received, but not for the age at treatment initiation and the proportion of patients having CNS metastasis in the treatment line matched non-eribulin group compared to patient level matched non-eribulin group. The variance ratio of all covariates covariates (except for the presence of CNS metastasis) was the most similar between the eribulin group and the treatment line matched non-eribulin group compared to the other non-eribulin groups.

Compared to the unmatched non-eribulin group, both the treatment line matched and the patient level matched non-eribulin groups had better covariate balance overall, expect for the age at treatment initiation.

The variance ratio should be as close as possible to 1 while the bootstrapped Kolmorogov Smirnov p-value should be maximized. 
Table S5.1: Balance statistics for each non eribulin group.

\begin{tabular}{|c|c|c|c|c|c|c|}
\hline Variable names & $\begin{array}{c}\text { Variance } \\
\text { ratio } \\
\text { unmatched } \\
\text { non-eribulin }\end{array}$ & $\begin{array}{l}\text { Variance } \\
\text { ratio after } \\
\text { treatment } \\
\quad \text { line } \\
\text { matching }\end{array}$ & $\begin{array}{l}\text { Variance } \\
\text { ratio after } \\
\text { patient } \\
\text { level } \\
\text { matching }\end{array}$ & $\begin{array}{c}\text { Bootstrapped } \\
\text { Kolmorogov } \\
\text { Smirnov p- } \\
\text { value } \\
\text { unmatched } \\
\text { non-eribulin }\end{array}$ & $\begin{array}{c}\text { Bootstrapped } \\
\text { Kolmorogov } \\
\text { Smirnov } p- \\
\text { value after } \\
\text { treatment line } \\
\text { matching } \\
\end{array}$ & $\begin{array}{c}\text { Bootstrapped } \\
\text { Kolmorogov } \\
\text { Smirnov } p \\
\text { value after } \\
\text { patient level } \\
\text { matching }\end{array}$ \\
\hline $\begin{array}{l}\text { Tumor subtype } \\
\text { (continuous) }\end{array}$ & 2.906 & 1.694 & 2.043 & 0.011 & 0.835 & 0.505 \\
\hline $\begin{array}{l}\text { Number of } \\
\text { previous } \\
\text { chemotherapy } \\
\text { (continuous) }\end{array}$ & 1.238 & 0.939 & 1.171 & 0.044 & 0.92 & 0.172 \\
\hline $\begin{array}{l}\text { Age at treatment } \\
\text { initiation }\end{array}$ & 0.597 & 0.837 & 0.543 & 0.617 & 0.22 & 0.297 \\
\hline $\begin{array}{l}\text { Presence of CNS } \\
\text { metastasis }\end{array}$ & 1.11 & 1.316 & 1.316 & NA & NA & NA \\
\hline
\end{tabular}

Abbreviations: CNS, central nervous system; NA, not applicable.

\section{QQ plots patient characteristics before and after matching}
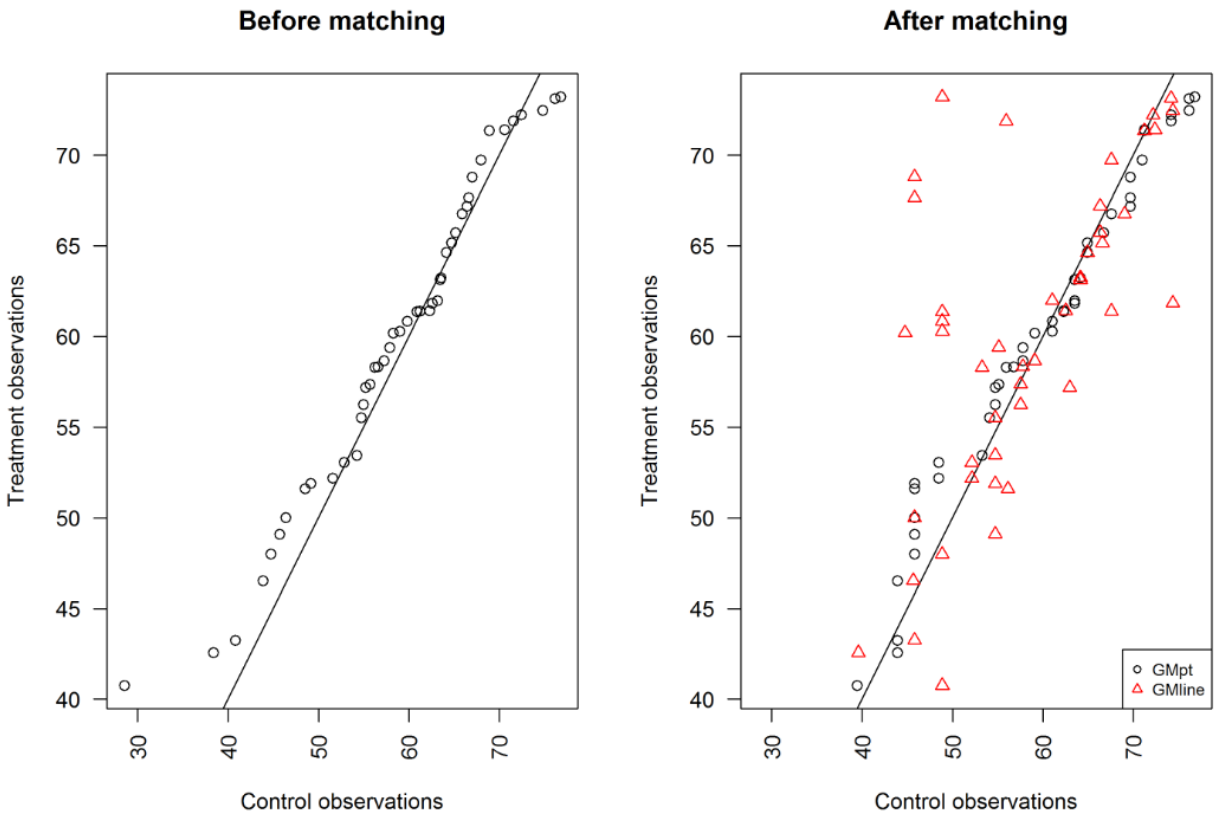

Figure S5. 3: QQplot of the age at treatment initiation of eribulin (treatment observation) versus noneribulin (control observations).

Abbreviations: GMline, treatment line matched non-eribulin group; GMpt, patient level matched noneribulin group; $Q Q$, quantile-quantile. 
Before matching

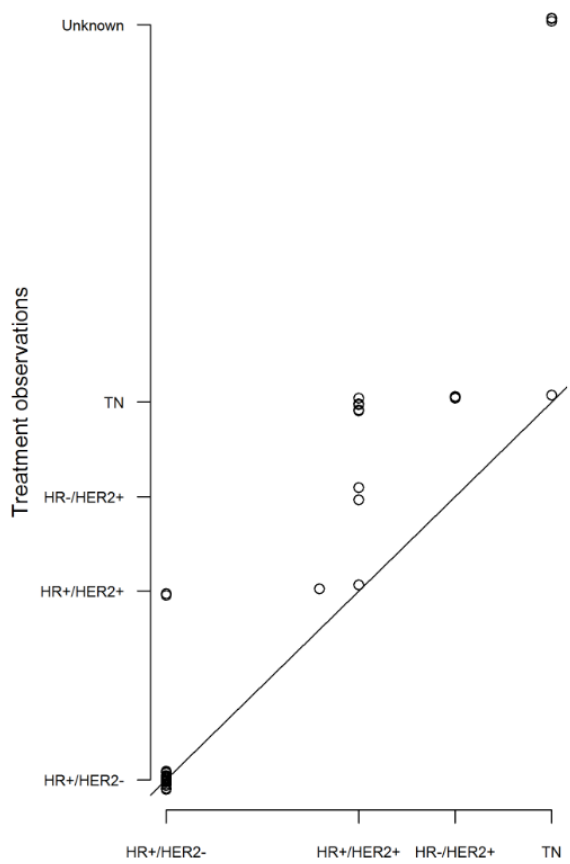

\section{After matching}

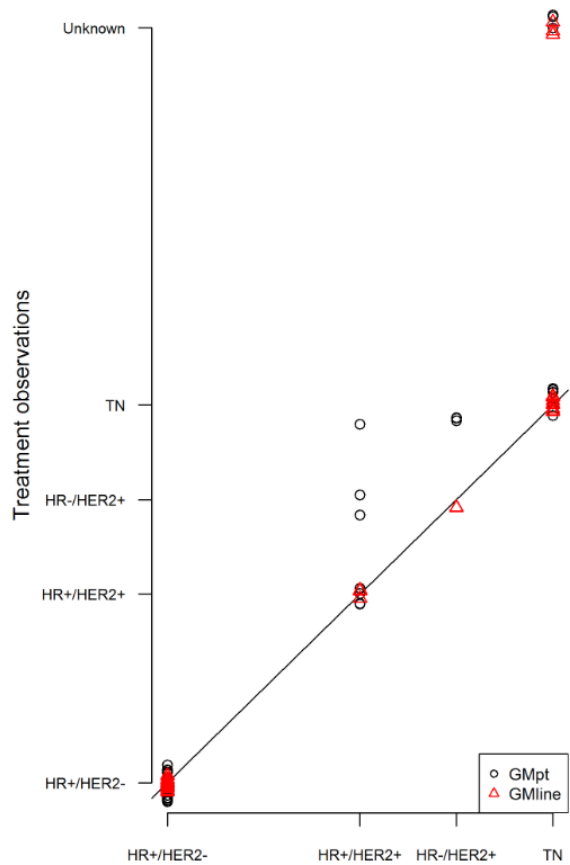

Figure S5.4: QQplot of the tumour subtype of eribulin (treatment observation) versus non-eribulin (control observations).

Abbreviation: HR, hormonal status; HER2, human epidermal growth factor receptor 2; GMline, treatment line matched non-eribulin group; GMpt, patient level matched non-eribulin group; $T N$, triple negative; $Q Q$, quantile-quantile. 
Before matching

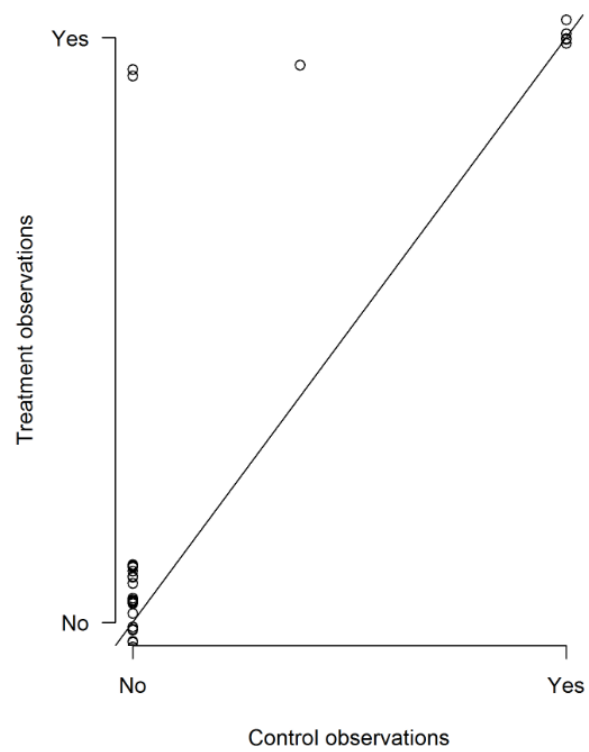

Before matching

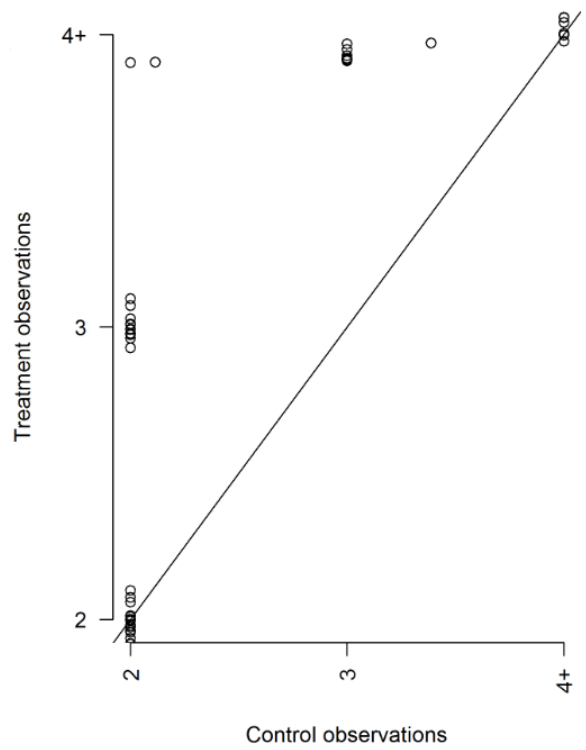

After matching

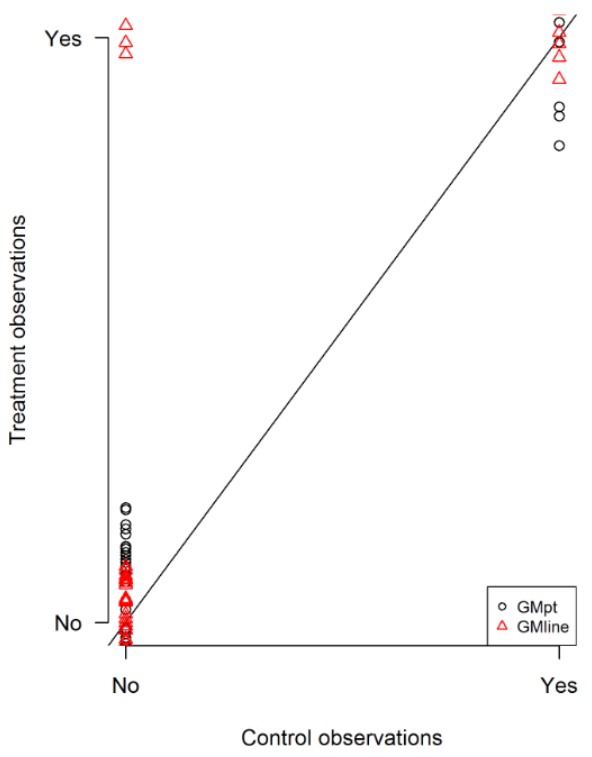

After matching

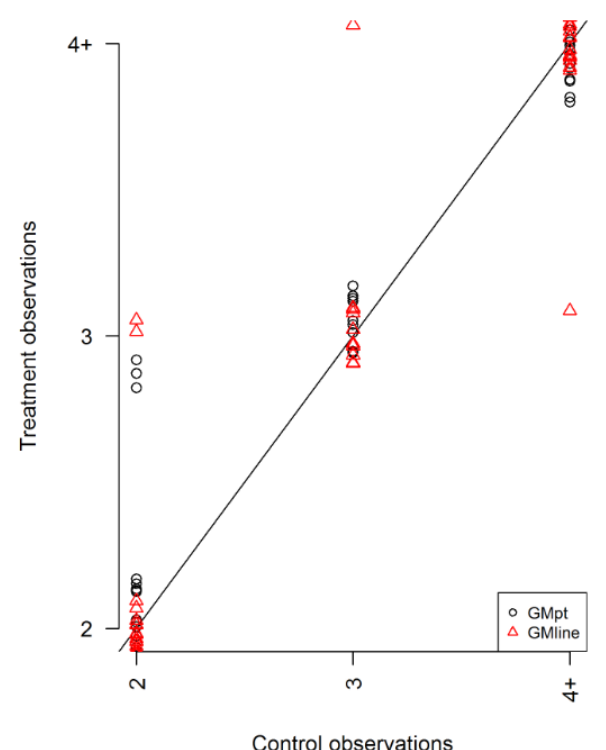

Figure S5.6: QQplot of the number of previous chemotherapy received in the eribulin (treatment observation) versus non-eribulin (control observations) group.

Abbreviation: GMline, treatment line matched non-eribulin group; GMpt, patient level matched non-eribulin group; $Q Q$, quantile-quantile. 


\section{Results of the sensitivity analysis: eribulin versus patient-level matched non-eribulin}

Table S5.2: Patient characteristics in each group.

\begin{tabular}{|c|c|c|c|c|c|}
\hline Treatment group & & \multirow{2}{*}{$\begin{array}{c}\text { Eribulin (N) } \\
45\end{array}$} & \multirow{2}{*}{$\%$} & \multirow{2}{*}{$\begin{array}{c}\begin{array}{c}\text { Patient level } \\
\text { matched non- } \\
\text { eribulin }\end{array} \\
45\end{array}$} & \multirow[t]{2}{*}{$\%$} \\
\hline Number of patient & & & & & \\
\hline Median age & & 61 & & 61 & \\
\hline Minimum age & & 41 & & 39 & \\
\hline Maximum age & & 73 & & 77 & \\
\hline \multirow[t]{5}{*}{ Tumour status } & $\mathrm{HR}+/ \mathrm{HER} 2-$ & 25 & $56 \%$ & 25 & $56 \%$ \\
\hline & $\mathrm{HR}+/ \mathrm{HER} 2+$ & 4 & $9 \%$ & 7 & $16 \%$ \\
\hline & HR-/HER2+ & 2 & $4 \%$ & 3 & $7 \%$ \\
\hline & Triple Negative & 11 & $24 \%$ & 10 & $22 \%$ \\
\hline & Unknown & 3 & $7 \%$ & 0 & 0 \\
\hline \multirow[t]{5}{*}{ Dominant metastatic site } & CNS & 10 & $22 \%$ & 10 & $22 \%$ \\
\hline & Visceral & 31 & $69 \%$ & 29 & $64 \%$ \\
\hline & Non-visceral & 4 & $9 \%$ & 6 & $13 \%$ \\
\hline & Bone only* & 2 & $4 \%$ & 6 & $13 \%$ \\
\hline & Unknown & 0 & $0 \%$ & 0 & $0 \%$ \\
\hline \multirow{5}{*}{$\begin{array}{l}\text { Number of metastatic } \\
\text { sites }\end{array}$} & 1 & 2 & $4 \%$ & 3 & $7 \%$ \\
\hline & 2 & 12 & $27 \%$ & 10 & $22 \%$ \\
\hline & 3 & 15 & $33 \%$ & 9 & $20 \%$ \\
\hline & 4 & 8 & $18 \%$ & 12 & $27 \%$ \\
\hline & $5+$ & 8 & $17 \%$ & 11 & $25 \%$ \\
\hline \multirow{5}{*}{$\begin{array}{l}\text { Number of previous } \\
\text { endocrine therapy in the } \\
A B C \text { setting }\end{array}$} & 0 & 16 & $36 \%$ & 16 & $36 \%$ \\
\hline & 1 & 7 & $16 \%$ & 6 & $13 \%$ \\
\hline & 2 & 7 & $16 \%$ & 9 & $20 \%$ \\
\hline & 3 & 5 & $11 \%$ & 7 & $16 \%$ \\
\hline & $4+$ & 10 & $22 \%$ & 7 & $15 \%$ \\
\hline \multirow{3}{*}{$\begin{array}{l}\text { Number of previous } \\
\text { chemotherapy in the } A B C \\
\text { setting }\end{array}$} & 2 & 15 & $33 \%$ & 18 & $40 \%$ \\
\hline & 3 & 13 & $29 \%$ & 10 & $22 \%$ \\
\hline & $4+$ & 17 & $37 \%$ & 17 & $38 \%$ \\
\hline
\end{tabular}

* Subgroup of non-visceral metastasis.

Abbreviations: $A B C$, advanced breast cancer; GM, genetic matched; HER2, human epidermal growth factor 2 receptor; HR, hormonal status 
A

Median PFS Eribulin $=3.5$ months $(95 \% \mathrm{Cl}: 2.7-5.5)$ Median PFS Non-eribulin $=2.7$ months $(95 \% \mathrm{Cl}: 1.8-4.7)$

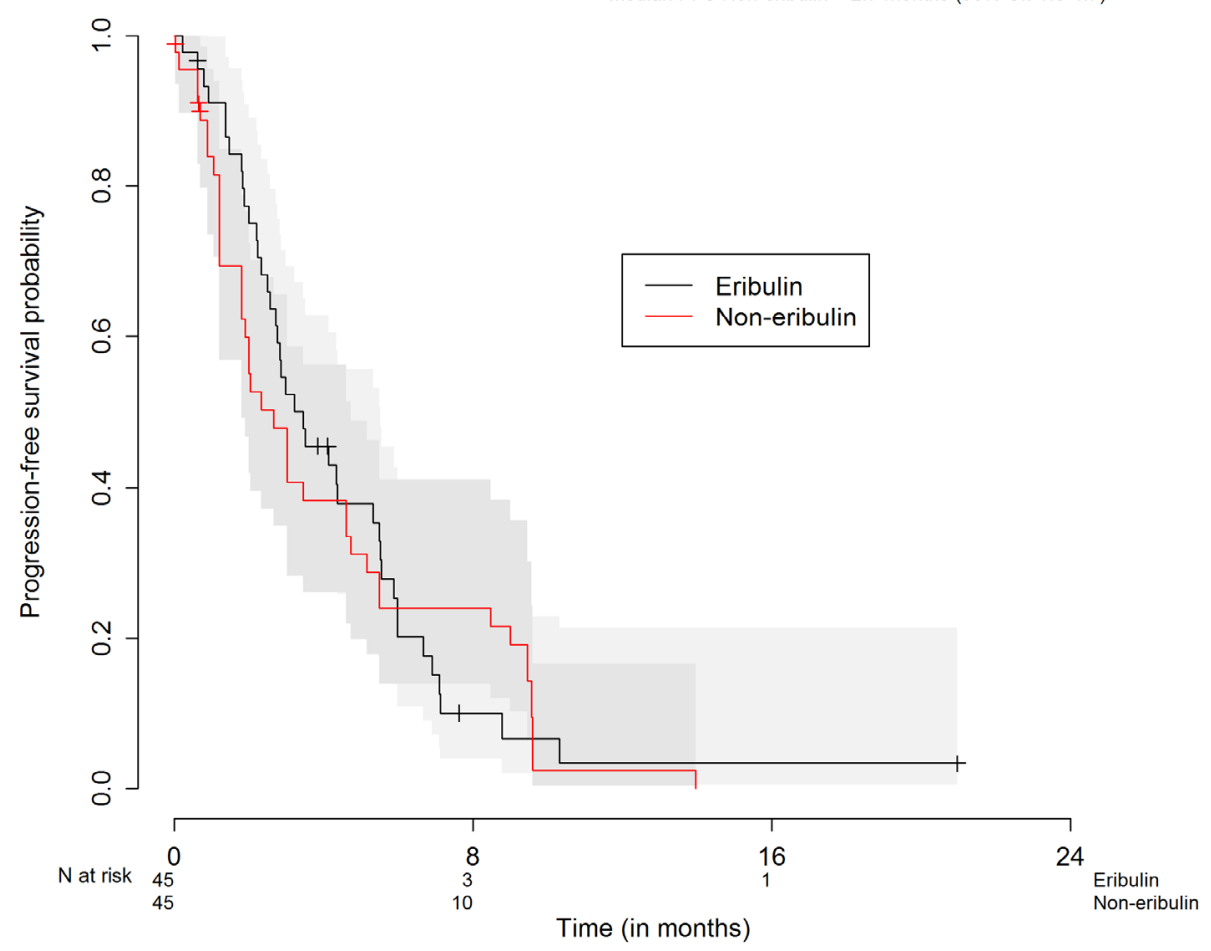

Figure S5.9: KM curves of PFS for eribulin and the patient level matched non-eribulin group. 
B

Median OS Eribulin $=5.9$ months $(95 \% \mathrm{Cl}: 4.6-11)$

Median OS Non-eribulin $=6.8$ months $(95 \% \mathrm{Cl}: 5.7-9.5)$

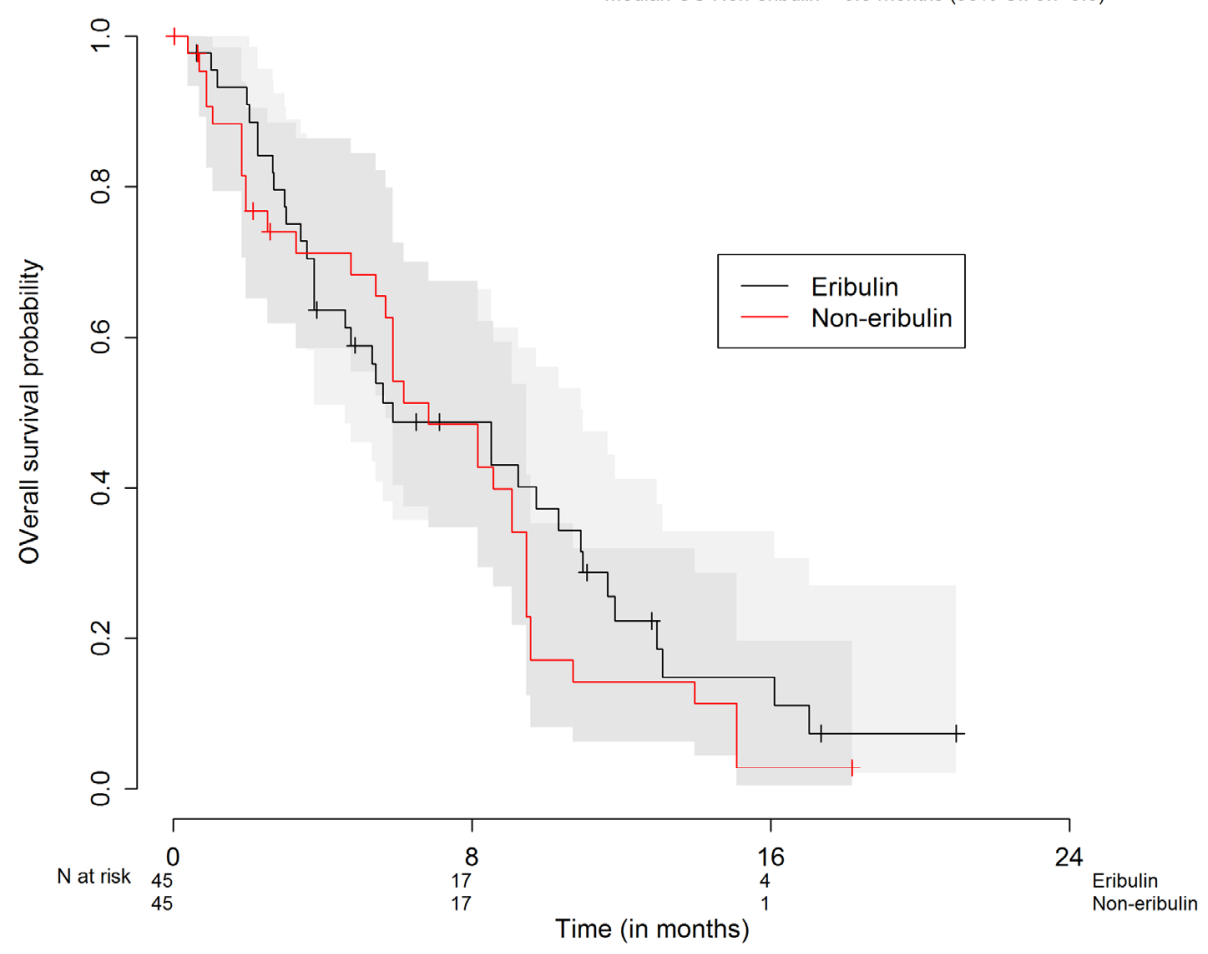

Figure S5.10: KM curves of OS for eribulin and the patient level matched non-eribulin group. 


\section{Results of the sensitivity analysis: eribulin versus unmatched non-eribulin}

Table S5.3: Patient characteristics in each group.

\begin{tabular}{|c|c|c|c|c|c|}
\hline Treatment group & & Eribulin (N) & $\%$ & $\begin{array}{c}\text { Unmatched non- } \\
\text { eribulin }\end{array}$ & $\%$ \\
\hline Number of patient & & 45 & & 74 & \\
\hline Median age & & 61 & & 60 & \\
\hline Minimum age & & 41 & & 29 & \\
\hline Maximum age & & 73 & & 77 & \\
\hline \multirow[t]{5}{*}{ Tumour status } & HR+/HER2- & 25 & $56 \%$ & 45 & $61 \%$ \\
\hline & $\mathrm{HR}+/ \mathrm{HER} 2+$ & 4 & $9 \%$ & 18 & $24 \%$ \\
\hline & HR-/HER2+ & 2 & $4 \%$ & 5 & $7 \%$ \\
\hline & Triple Negative & 11 & $24 \%$ & 6 & $8 \%$ \\
\hline & Unknown & 3 & $7 \%$ & 0 & 0 \\
\hline \multirow{5}{*}{$\begin{array}{l}\text { Dominant metastatic } \\
\text { site }\end{array}$} & CNS & 10 & $22 \%$ & 12 & $16 \%$ \\
\hline & Visceral & 31 & $69 \%$ & 52 & $70 \%$ \\
\hline & Non-visceral & 4 & $9 \%$ & 10 & $14 \%$ \\
\hline & Bone only* & 2 & $4 \%$ & 9 & $12 \%$ \\
\hline & Unknown & 0 & $0 \%$ & 0 & $0 \%$ \\
\hline \multirow{5}{*}{$\begin{array}{l}\text { Number of metastatic } \\
\text { sites }\end{array}$} & 1 & 2 & $4 \%$ & 12 & $16 \%$ \\
\hline & 2 & 12 & $27 \%$ & 17 & $23 \%$ \\
\hline & 3 & 15 & $33 \%$ & 18 & $24 \%$ \\
\hline & 4 & 8 & $18 \%$ & 14 & $19 \%$ \\
\hline & $5+$ & 8 & $17 \%$ & 13 & $17 \%$ \\
\hline \multirow{5}{*}{$\begin{array}{l}\text { Number of previous } \\
\text { endocrine therapy in } \\
\text { the } A B C \text { setting }\end{array}$} & 0 & 16 & $36 \%$ & 20 & $27 \%$ \\
\hline & 1 & 7 & $16 \%$ & 13 & $18 \%$ \\
\hline & 2 & 7 & $16 \%$ & 13 & $18 \%$ \\
\hline & 3 & 5 & $11 \%$ & 16 & $22 \%$ \\
\hline & $4+$ & 10 & $22 \%$ & 12 & $16 \%$ \\
\hline Number of previous & 2 & 15 & $33 \%$ & 49 & $66 \%$ \\
\hline chemotherapy in the & 3 & 13 & $29 \%$ & 13 & $18 \%$ \\
\hline ABC setting & $4+$ & 17 & $37 \%$ & 12 & $16 \%$ \\
\hline
\end{tabular}

* Subgroup of non-visceral metastasis.

Abbreviations: $A B C$, advanced breast cancer; GM, genetic matched; HER2, human epidermal growth factor 2 receptor; HR, hormonal status. 
Median PFS Eribulin $=3.5$ months $(95 \% \mathrm{Cl}: 2.7-5.5)$ Median PFS Non-eribulin $=3.4$ months $(95 \% \mathrm{Cl}: 2.3-5.2)$

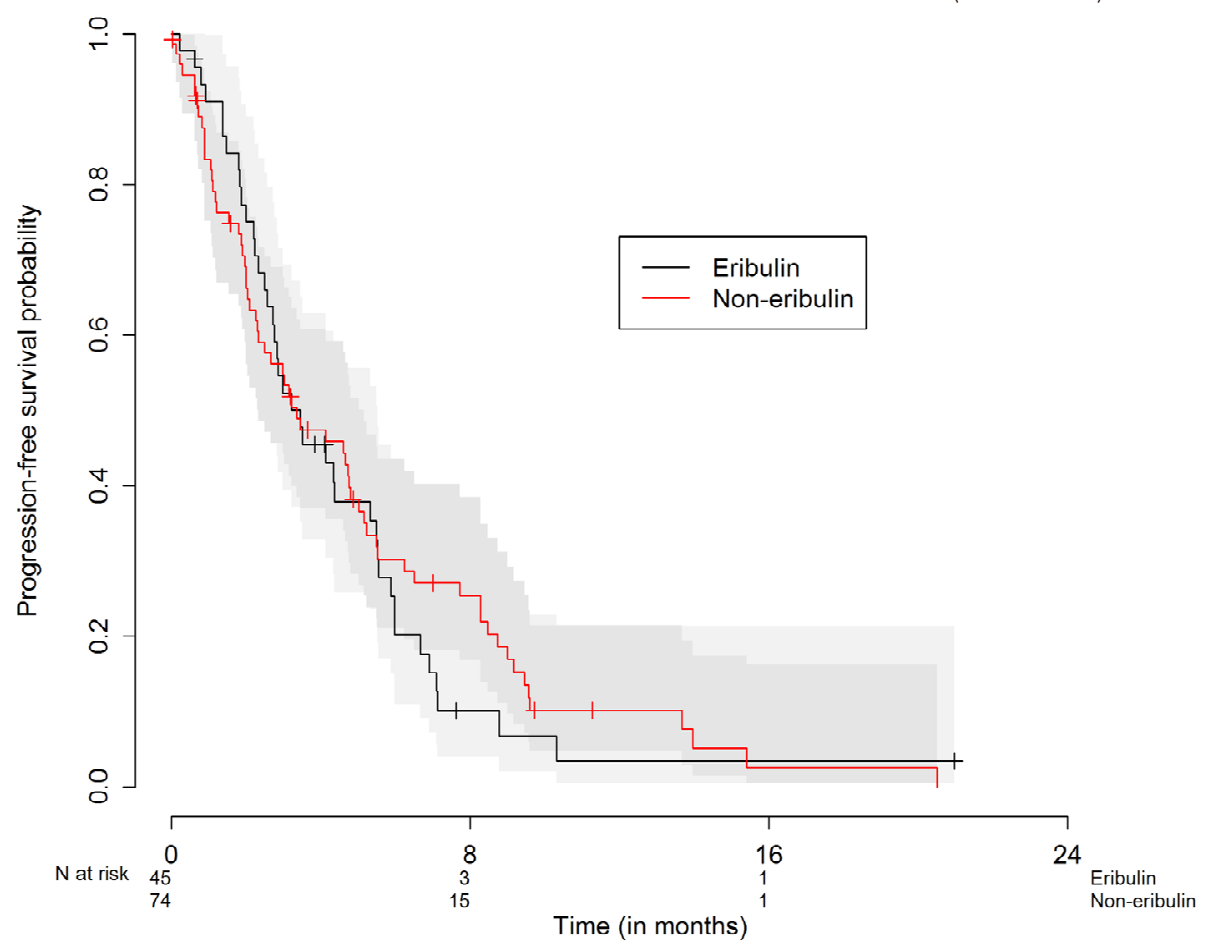

Figure S5.11: KM curves of PFS for eribulin and the unmatched non-eribulin group. 
C

Median OS Eribulin $=5.9$ months $(95 \% \mathrm{Cl}: 4.6-11)$

Median OS Non-eribulin $=8.2$ months $(95 \% \mathrm{Cl}: 5.9-11)$

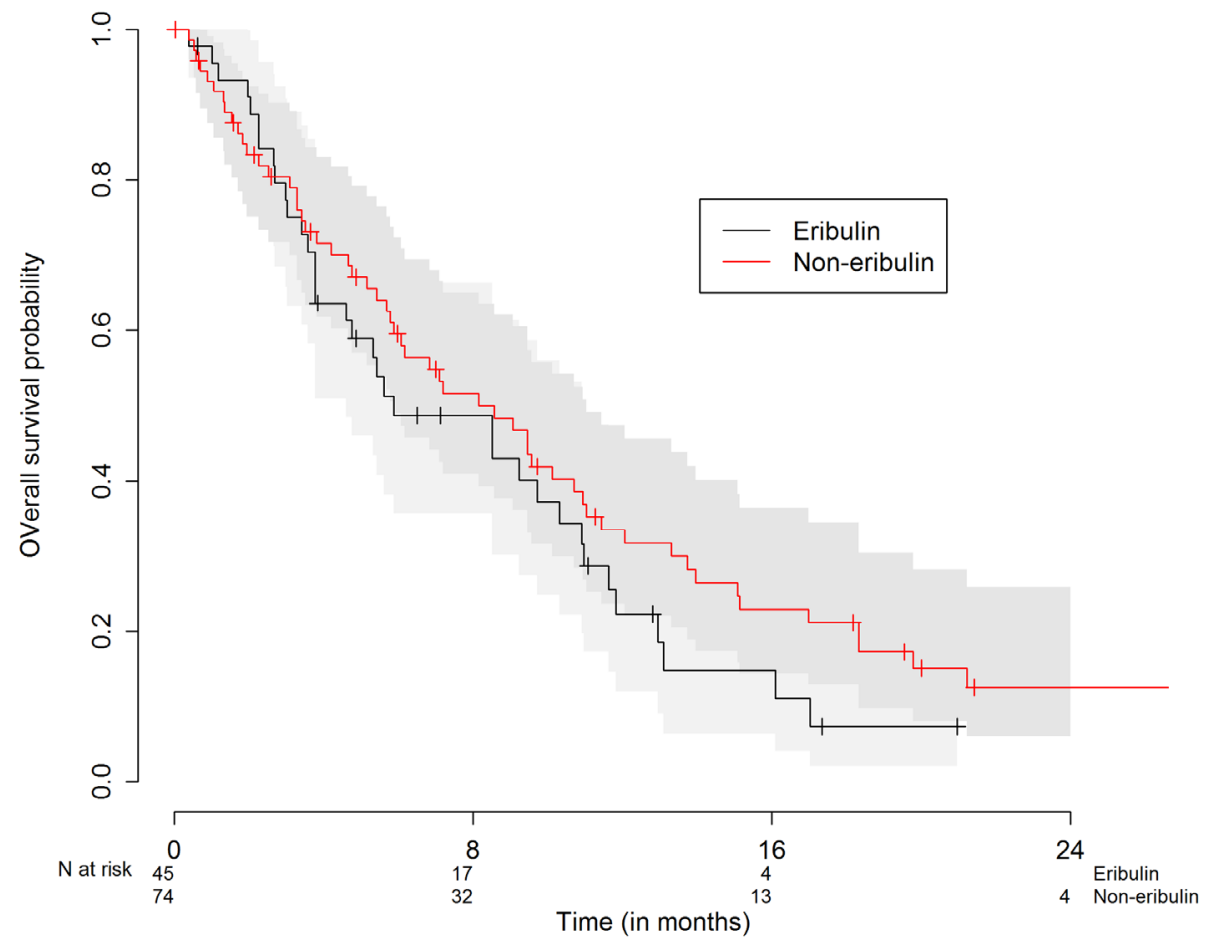

Figure S5.12: KM curves of OS for eribulin and the unmatched non-eribulin group. 


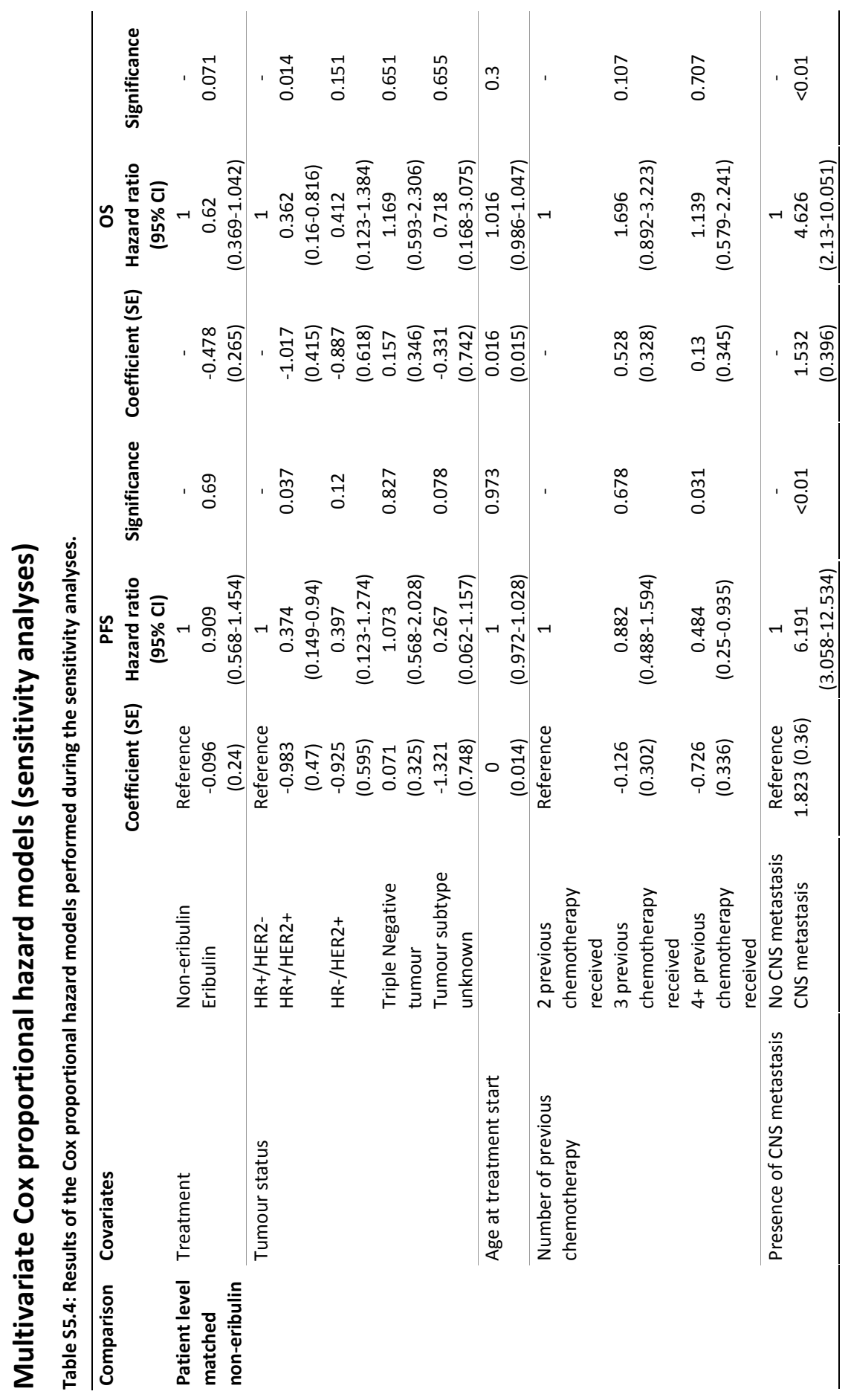




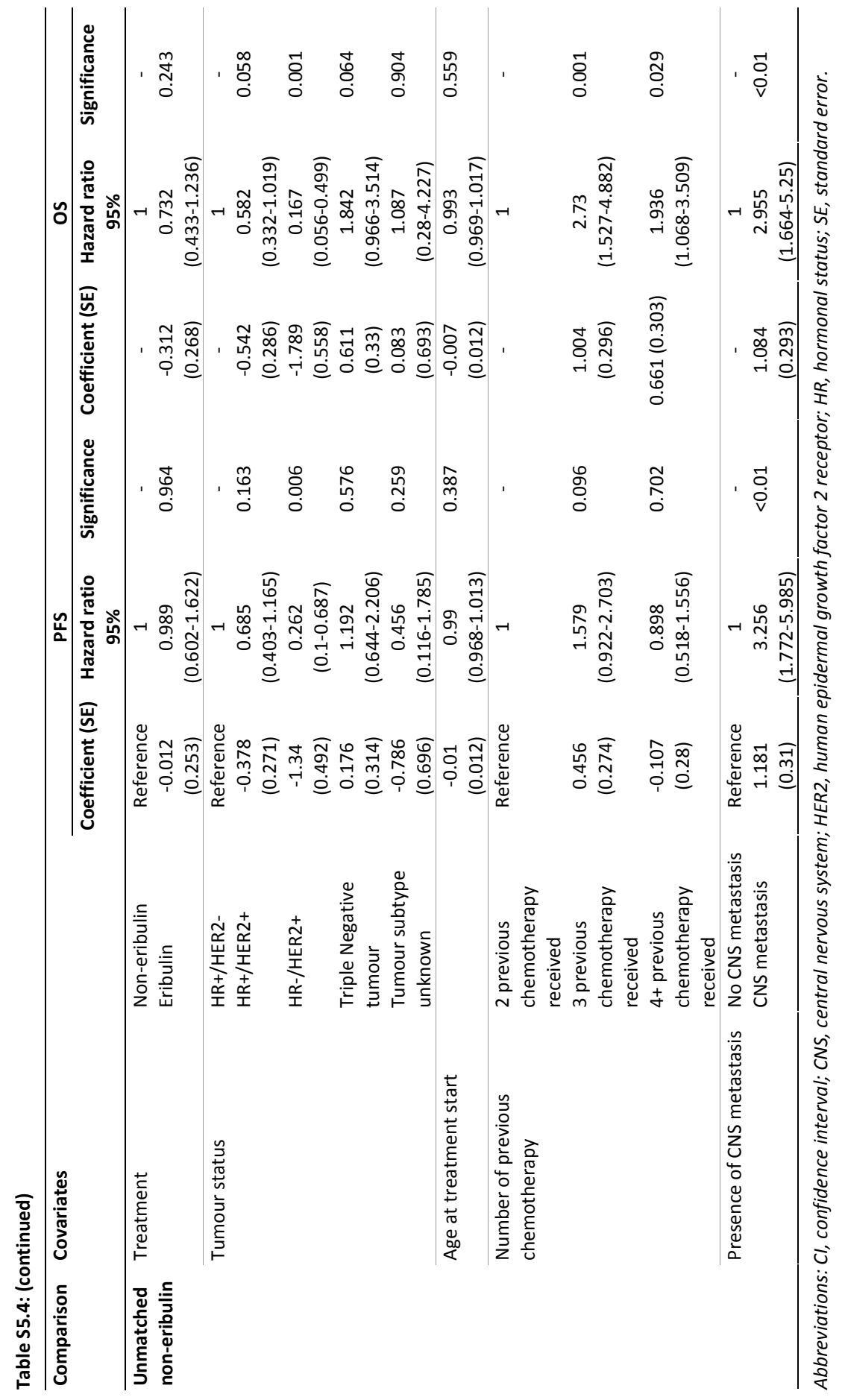




\section{Univariate Cox proportional hazard models}

Table S5.5: Cox proportional hazard model results PFS - eribulin versus treatment line matched noneribulin.

\begin{tabular}{llll}
\hline Covariate & Coefficient $(\mathrm{SE})$ & Hazard ratio $(95 \% \mathrm{Cl})$ & Significance \\
\hline Eribulin & $0.07(0.232)$ & $1.073(0.681-1.692)$ & 0.762 \\
\hline
\end{tabular}

Abbreviations: $\mathrm{Cl}$, confidence interval; SE, standard error.

Table S5.6: Cox proportional hazard model results OS - eribulin versus treatment line matched noneribulin

\begin{tabular}{llll}
\hline Covariate & Coefficient (SE) & Hazard ratio $(95 \% \mathrm{CI})$ & Significance \\
\hline Eribulin & $-0.28(0.254)$ & $0.755(0.459-1.243)$ & 0.27 \\
\hline
\end{tabular}

Abbreviations: $\mathrm{Cl}$, confidence interval; SE, standard error.

Table S5.7: Cox proportional hazard model results PFS - eribulin versus patient level matched noneribulin.

\begin{tabular}{lccc}
\hline Covariate & Coefficient $(\mathrm{SE})$ & Hazard ratio $(95 \% \mathrm{Cl})$ & Significance \\
\hline Eribulin & $-0.096(0.225)$ & $0.908(0.584-1.413)$ & 0.67 \\
\hline
\end{tabular}

Abbreviations: $\mathrm{Cl}$, confidence interval; $\mathrm{SE}$, standard error.

Table S5.8: Cox proportional hazard model results OS - eribulin versus patient level matched non-eribulin.

\begin{tabular}{lccc}
\hline Covariate & Coefficient $(\mathrm{SE})$ & Hazard ratio $(95 \% \mathrm{Cl})$ & Significance \\
\hline Eribulin & $-0.251(0.24)$ & $0.778(0.486-1.245)$ & 0.295 \\
\hline
\end{tabular}

Abbreviations: $\mathrm{Cl}$, confidence interval; $S E$, standard error.

Table S5.9: Cox proportional hazard model results PFS - eribulin versus unmatched non-eribulin.

\begin{tabular}{llll}
\hline Covariate & Coefficient (SE) & Hazard ratio $(95 \% \mathrm{Cl})$ & Significance \\
\hline Eribulin & $0.092(0.204)$ & $1.097(0.735-1.636)$ & 0.651 \\
\hline
\end{tabular}

Abbreviations: $\mathrm{Cl}$, confidence interval; SE, standard error.

Table S5.10: Cox proportional hazard model results OS - eribulin versus unmatched non-eribulin.

\begin{tabular}{lccc}
\hline Covariate & Coefficient $(\mathrm{SE})$ & Hazard ratio $(95 \% \mathrm{Cl})$ & Significance \\
\hline Eribulin & $0.259(0.22)$ & $1.295(0.842-1.993)$ & 0.239 \\
\hline
\end{tabular}

Abbreviations: $\mathrm{Cl}$, confidence interval; SE, standard error. 


\section{Chapter 6}

\section{An economic evaluation of eribulin for advanced breast cancer treatment based on the Southeast Netherlands advanced breast cancer registry}

Xavier G.L.V. Pouwels, Bram L.T. Ramaekers, Sandra M.E. Geurts, Frans Erdkamp, Birgit E.P.J. Vriens, Kirsten N.A. Aaldering, Yes A.J. van de Wouw, M.W. Dercksen, Tineke J. Smilde, Natasha A.J.B. Peters, Anne-Marie van Riel, Manon J. Pepels, José Heijnen-Mommers, Vivianne C.G. Tjan-Heijnen, Maaike de Boer, Manuela A. Joore 


\section{Abstract}

\section{Background}

In 2013, eribulin was reimbursed under a coverage with evidence development (CED) as third or later chemotherapy line for advanced breast cancer $(A B C)$ patients in the Netherlands because of uncertain cost effectiveness. In 2016, the final decision of reimbursing eribulin was taken without considering the evidence collected during CED research. We analysed the cost effectiveness of eribulin versus non-eribulin chemotherapy, using real-world data.

\section{Methods}

A three health states (progression-free, progressed disease, dead) partitioned survival model was developed. The SOuth East Netherlands Advanced BREast Cancer (SONABRE) registry informed the effectiveness and costs inputs. Health state utility values were obtained from the literature. Incremental cost effectiveness ratio (ICER) between the eribulin and matched noneribulin chemotherapy was estimated. Deterministic and probabilistic sensitivity analyses, and scenario analyses were performed. The financial risk (i.e. the expected value of perfect information (EVPI) plus the expected monetary loss (eML) associated with reimbursing eribulin) and budget impact associated with reimbursing eribulin were calculated.

\section{Results}

Eribulin led to higher health benefits ( 0.07 quality-adjusted life year (QALY)) and costs (€15,321) compared with non-eribulin chemotherapy. This resulted in an ICER of $€ 220,608$. At a $€ 80,000$ per QALY threshold, the risk of reimbursing eribulin was $€ 9,791$ per patient (EVPI €13, eML $€ 9,778$ ). Scaled up to the Dutch population, the estimated annual budget impact was $€ 1.9$ million and the annual risk of reimbursing eribulin was $€ 2.7$ million.

\section{Conclusion}

From a Dutch societal perspective, eribulin is not cost effective when considering its list price as third and later chemotherapy line for $A B C$ patients. 


\section{Introduction}

In 2013, eribulin mesylate (eribulin) as third (or later) chemotherapy line for advanced breast cancer $(A B C)$ treatment became available in the Netherlands through coverage with evidence development (CED). ${ }^{1}$ CED schemes were introduced by the Dutch Healthcare Institute (Zorginstituut Nederland; ZIN) in 2006 to regulate the access to expensive drugs with uncertain (cost) effectiveness. During CED schemes, treatments are conditionally reimbursed during a pre-defined period of time, often four years, while research is performed to reduce the uncertainty surrounding their cost effectiveness. Definitive reimbursement decision are taken after CED research.

Eribulin was reimbursed through CED because it statistically significantly improved overall survival (OS) by 2.5 months compared with treatment of physicians' choice (TPC) in the EMBRACE trial ${ }^{2}$, but its value for money was unfavourable and uncertain. ${ }^{1}$ In 2013, the estimated incremental cost-effectiveness ratio (ICER; ratio of incremental costs and incremental benefits) of eribulin by the company was above $€ 145,000$ per quality-adjusted life year (QALY) gained. ${ }^{1}$ The most important uncertainties were the unavailability of Dutch utility values, the resource use associated with eribulin treatment, and the effectiveness of the comparator treatments within clinical practice. CED research commissioned by ZIN focused on obtaining this evidence within the Dutch setting. ZIN also advised the Ministry of Health, Wellbeing and Sport to negotiate a financial arrangement to limit the financial risk of reimbursing eribulin during research.

In December 2013, ZIN developed a 'risk-oriented insurance package management'. This strategy prescribes an assessment of technologies representing a risk for the affordability, accessibility, and quality of the basic insurance package, meaning that a formal clinical and cost-effectiveness assessment is performed only for drugs with an established added therapeutic value and an estimated annual budget impact (BI) of at least $€ 2.5$ million. ${ }^{3}$ Since the estimated BI incurred by eribulin in the Netherlands was lower than $€ 2.5$ million (calculations not publicly available), eribulin was recommended in 2016 by ZIN as third (or later) treatment line for ABC treatment, without assessing the evidence collected during CED research. ${ }^{4}$

We analysed the cost effectiveness and $\mathrm{BI}$ of eribulin versus non-eribulin chemotherapy from a societal perspective using Dutch real-world data. 


\section{Methods}

\subsection{Scope of the evaluation}

This study examined the cost effectiveness of eribulin versus non-eribulin chemotherapy as third or later chemotherapy line for $A B C$ treatment. Eligible patients for eribulin treatment were identified in the SOuth East Netherlands Advanced BREast Cancer (SONABRE) registry (NCT03577197). The SONABRE registry contains patient and treatment information (patient and tumor characteristics, treatments administered including dose and toxicity) of patients diagnosed with ABC from 2007 onwards in 12 hospitals in the Southeast of the Netherlands. Additionally, the date of progression and death, and other health care use (outpatients visits, imaging, blood transfusions, punctures, hospital admissions) are collected in the SONABRE registry. The Medical Research Ethics Committee of the Maastricht University Medical Centre+ approved the establishment of the registry (No. 15-4-239). Data lock was on October $23^{\text {rd }}, 2017$.

Patients were eligible for eribulin when they had received at least two previous chemotherapy lines for $A B C$ treatment, including prior anthracycline- and a taxanebased treatments. Eligible patients who received eribulin composed the eribulin group and eligible patients who received another chemotherapy composed the non-eribulin group. The non-eribulin group was matched to the eribulin group using Genetic Matching on treatment lines to minimise the potential bias induced by confounding by indication) (supplemental material 1). Eribulin was administered on days 1 and 8 of a 21 day cycle at a $1.23 \mathrm{mg} / \mathrm{m}^{2}$ dose.

Both eribulin and matched non-eribulin groups included 45 patients. Their mean age was 59 years old. In both groups, the majority of patients had hormone receptor positive and human epidermal growth factor receptor 2 negative tumours (56\% in both groups). The median number of metastatic sites was 3 and visceral metastases were the predominant metastatic site in both groups. Additionally, respectively $22 \%$ and $16 \%$ of patients in the eribulin ans non-eribulin groups suffered from central nervous system metastasis. Patients had received a median of three previous chemotherapy regimens for $A B C$. Sixty-seven percent of patients received eribulin as fourth or later line. The chemotherapy that were administered to patients in the noneribulin group were: capecitabine (24\%), vinorelbine (22\%), carboplatin (13\%), doxorubicin in non pegylated liposomes (11\%), and gemcitabine (7\%). ${ }^{5}$ Table 56.2 of the Supplemental material provides a complete overview of the treatment administered to patients in in the non-eribulin group. Full details on patients characteristics are provided in Pouwels et al.. ${ }^{5}$ 
A lifetime horizon (5 years) and a weekly cycle were implemented. The analysis took the Dutch societal perspective, effects and costs were discounted according to Dutch guidelines (Table 6.1). ${ }^{6}$ Outcomes were costs, life years (LYs), and quality-adjusted life years (QALYS).

\subsection{Model structure}

A three health-states partitioned survival model (progression-free (PF), progressed disease (PD) and death) was developed in $\mathrm{R}$ (executable model and model structure figure in supplementary material). ${ }^{7,8}$ Patients entered the model in the PF health state. During each model cycle, patients could either progress or die, or remain PF. Patients who progressed could die but could not become PF again.

The proportion of patients in each health state of this partitioned survival model is determined by two parametric survival models, one estimating progression-free survival (PFS) and one estimating OS. The proportion of patients in the PF health state was determined as the area under de curve of the PFS survival model while the proportion of patients in the death health state was estimated as the opposite of the area under the curve of the OS survival model (1-OS). Finally, the proportion of patients who were neither PF nor dead populated the PD health state [PD=(1-PFS)(1-OS)]. This was estimated by first calculating the proportion of patients who did not experience progression or died (1-PFS) and then subtracting the proportion of patients who died from this number (1-OS) (Figure 6.1). Minimum functions were implemented to prevent progression-free survival (PFS) to become larger than OS and to prevent time to treatment discontinuation (TTD) to become larger than PFS (clinical opinion specified treatment would be discontinued upon progression). TTD is used to estimate treatment costs as described in the following section and does not affect the proportion of patients in any health state.

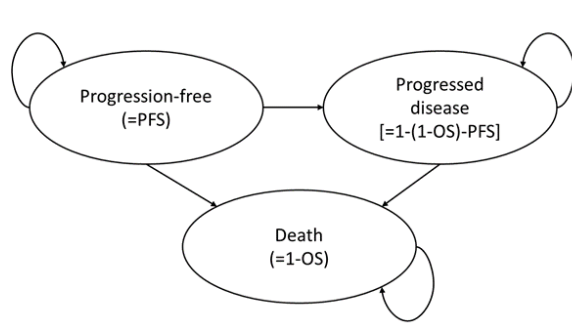

Figure 6.1: Model structure

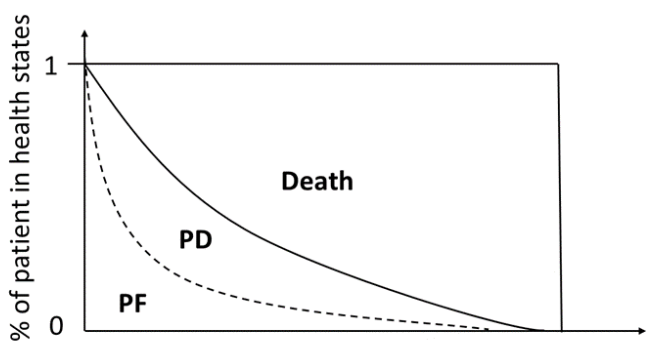

Time 


\subsection{Model inputs}

\subsubsection{Effectiveness and adverse events}

Multiple parametric survival models were fitted to PFS and OS individual patient data of the eribulin and matched non-eribulin groups. ${ }^{5}$ Visual inspection, statistical fit, and clinical expert opinion informed survival models selection for the base-case analysis (supplemental material 1). ${ }^{9}$ Stratified parametric survival models with log normal distributions, fitted to the PFS data, informed the proportion of patients in the PF health state (Figure 6.2A). Stratified parametric survival models with gamma distributions, fitted to the OS data, informed the proportion of deceased patients (Figure 6.2B).

The proportions of eribulin and non-eribulin patients who underwent hospitalisation due to adverse events (AEs) informed the probability of AEs in each group (Table 6.1). Total costs and disutility incurred by AEs were accrued once at the beginning of the model.

\subsubsection{Utility values}

Health state utility values were obtained from a cross-sectional study performed in a subset of patients included in SONABRE (Table 6.1). ${ }^{10}$ Patients filled in the EuroQol-5 dimensions 3-levels (EQ-5D-3L) during outpatient visits. EQ-5D scores were valued via the tariff developed by Dolan et al. ${ }^{11}$ These utility values were selected because they were obtained from patients from the region covered by SONABRE. The disutility associated with febrile neutropenia elicited in the UK general population through the EQ-5D-3L was used as AE disutility value. ${ }^{12}$

\subsubsection{Resource and costs}

Chemotherapy costs in the PF health state were estimated based on the eribulin and matched non-eribulin groups. The costs of eribulin considered vial waste. The costs of the non-eribulin chemotherapy were estimated based on the mean dose administered to patients. Total non-eribulin chemotherapy costs were aggregated per administration schedule and weighted by the proportion of patients receiving each chemotherapy (Table 6.1). Chemotherapy costs of all treatments were adjusted for dose intensity.

TTD survival curves, fitted to eribulin and non-eribulin patient data, estimated the proportion of patients receiving chemotherapy during each model cycle in the PF health state. TTD survival curves were selected through the same algorithm as the PFS and OS curves. ${ }^{9}$ Stratified parametric survival models with log-logistic distributions estimated TTD (Figure 6.2C). Intravenous administration costs and chemotherapy preparation costs were implemented (Table 6.1). 
A

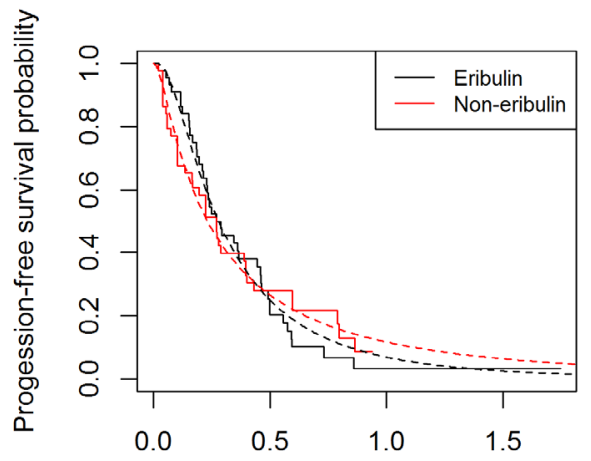

Time (in years)

B

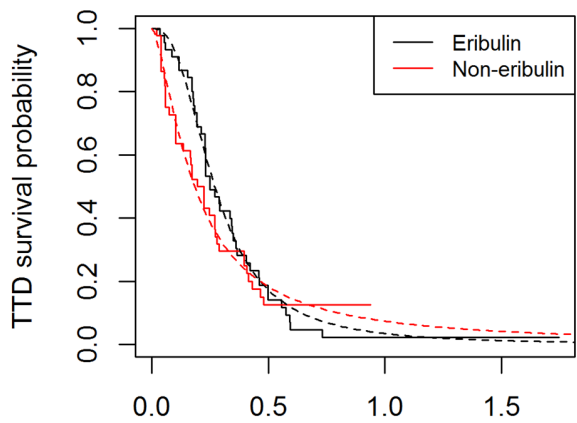

Time (in years)

C

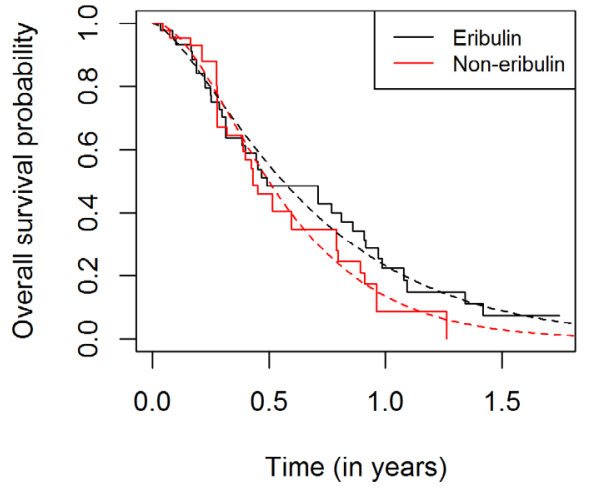

Figure 6.2: Kaplan-Meier curve and estimated survival for (A) progression-free survival (log normal distribution), (B) time to treatment discontinuation (log-logistic distribution), and (C) overall survival (gamma distribution).

Abbreviation: TTD, time to treatment discontinuation. 
In the PD state, treatment-independent weekly systemic treatment costs were estimated based on the mean dose and duration of treatments administered after eribulin and non-eribulin chemotherapy (Table 6.1). Chemotherapy prices were obtained from medicijnkosten. nl. $^{13}$

Treatment-independent outpatient visits, imaging, blood transfusions, radiotherapy, surgery, and hospitalisation costs for the PF and PD health states were estimated based on resource use observed in the eribulin and matched non-eribulin groups (Table 6.1). Costs were calculated by multiplying resources used by their prices. Prices were obtained from multiple sources; when publicly unavailable, internal prices were obtained (i.e. Maastricht University Medical Centre+) and cannot be reported (supplemental material 1 explains costs estimation). Age-adjusted related and unrelated health care costs were estimated through the Practical Application to Include future Disease costs (PAID) v1.1. ${ }^{14}$ Due to the short time horizon, we only considered unrelated health care costs of living an additional year of life (Table 6.1).

Travel costs to the hospital for intravenous administrations and hospitalisations due to AEs were included in the model (supplemental material 2, Section 2.7; Table 6.1). Productivity losses were not included.

All prices and costs were converted to $€ 2018$ using consumer price indexes. ${ }^{15}$

\subsection{Analyses}

Total costs and QALY were calculated for the eribulin and non-eribulin groups, the ICER was obtained by dividing the incremental costs by the incremental QALY of eribulin. Deterministic results were calculated by using mean values of parameter estimates. Deterministic one-way sensitivity analyses were performed to investigate the influence of varying each parameter value individually on the results. Hence, discount rates, survival model parameters, length and probability of adverse events, and costs and quality of life inputs were varied individually within a plausible range of values and the results of these variations were recorded. The plausible range of values was determined by the $95 \%$ confidence interval of model inputs, when available. If the $95 \%$ confidence interval was not available, it was calculated by assuming a $25 \%$ standard error.

Additionally, scenario analyses investigated the influence of using (a) vial sharing for eribulin, (b) no unrelated health care costs in the last year of life, (c) alternative parametric survival models for PFS, OS and TTD, (d) utility values from another costeffectiveness analysis ${ }^{16}$, (e) the relative effectiveness of EMBRACE, and ( $f$ ) a hospital perspective. 


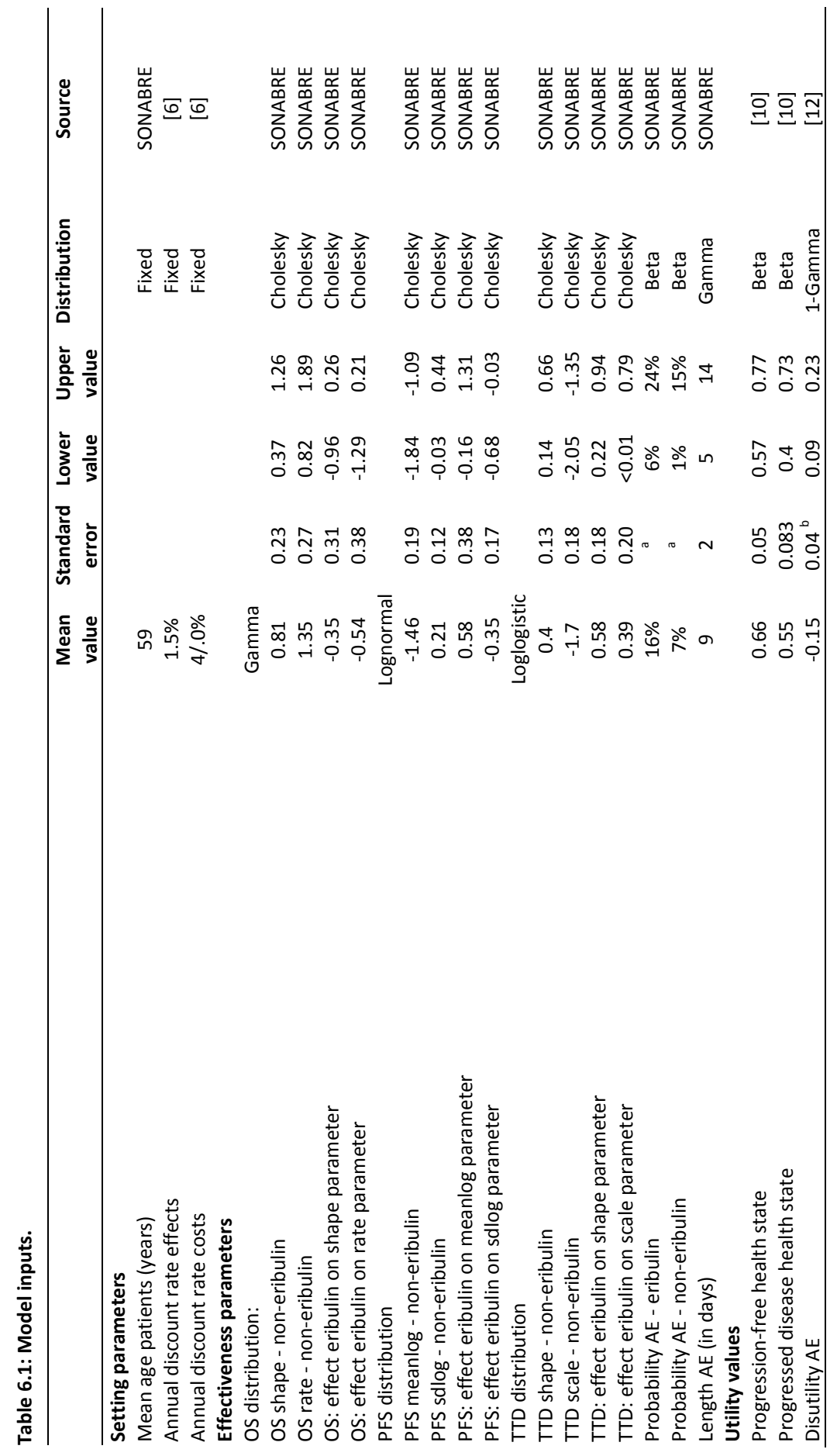




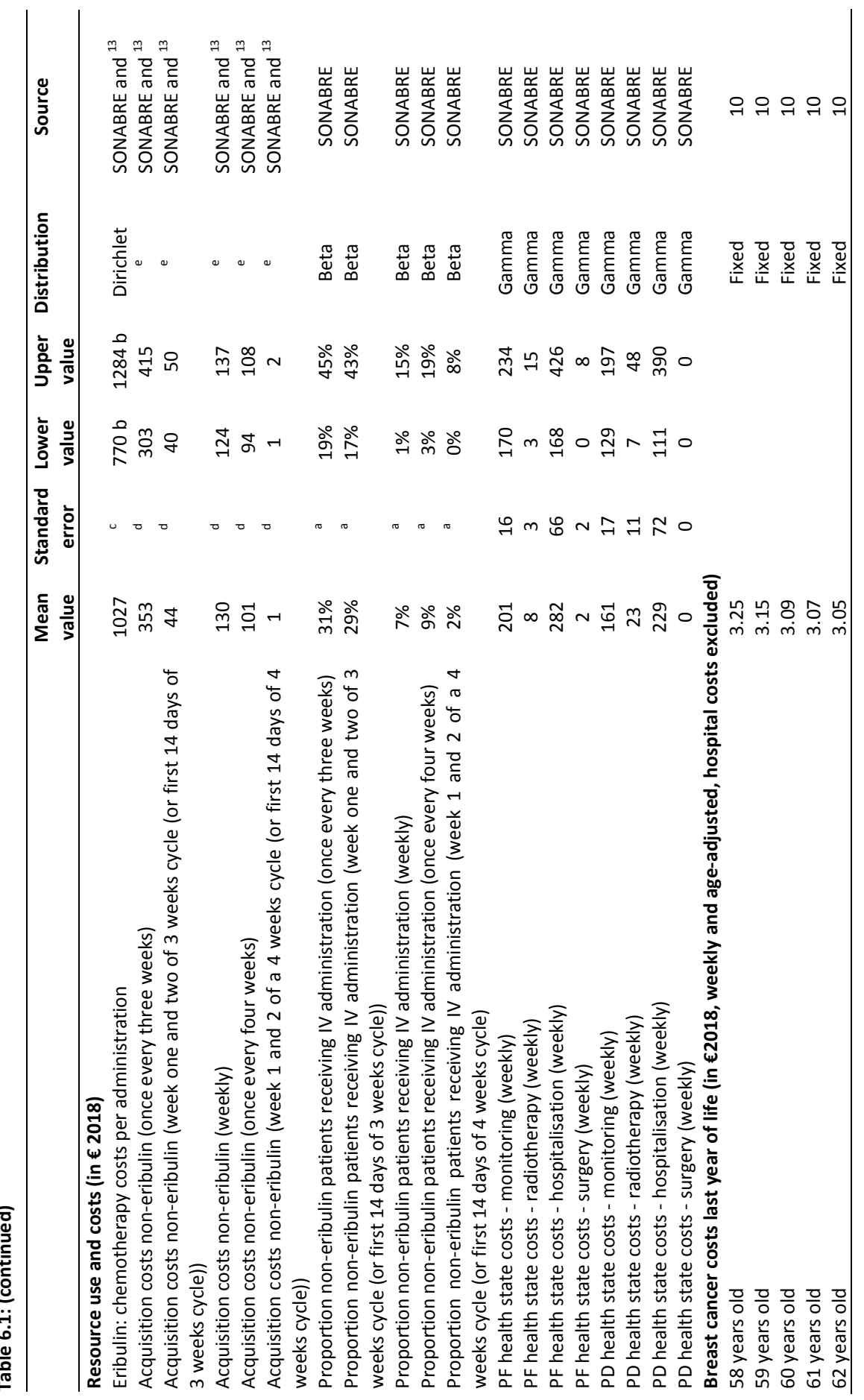




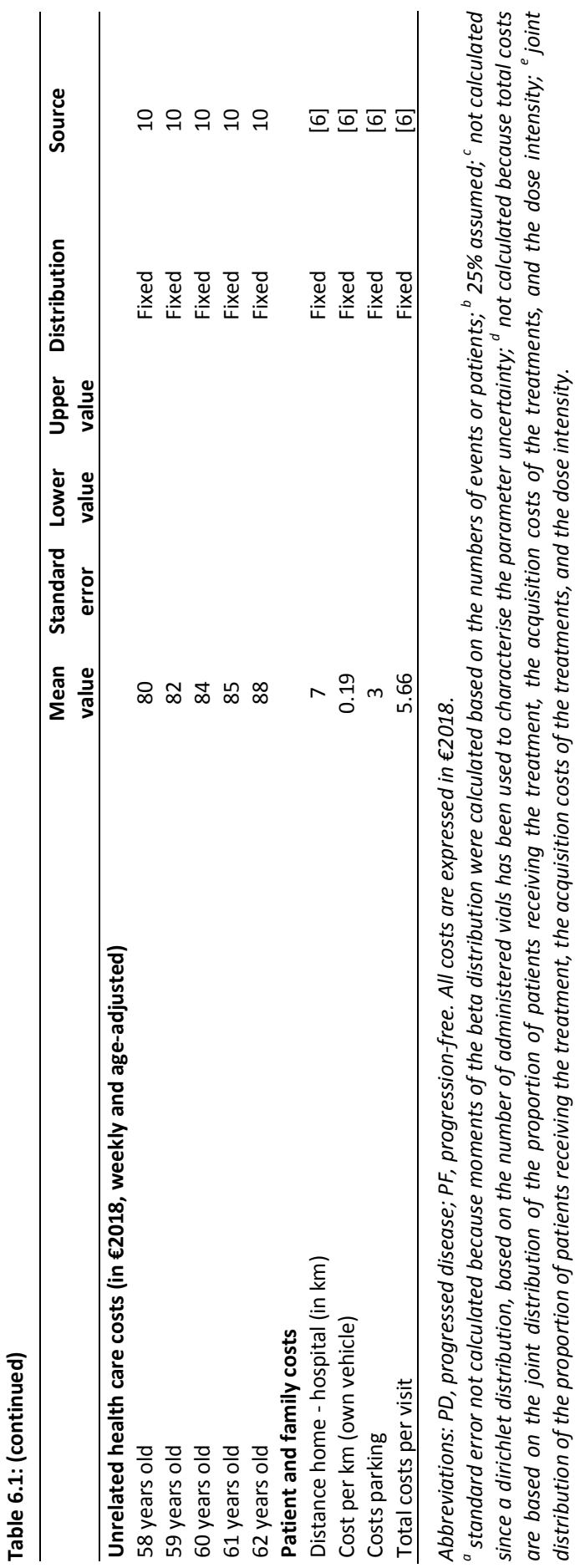


A probabilistic sensitivity analysis (PSA), capturing the influence of the joint parameter uncertainty on the results, was performed via Monte Carlo simulation. During this simulation, 10,000 random values for each parameter were drawn from pre-assigned distributions (Table 6.1). Probabilistic results were used to calculate the price at which eribulin would become cost effective, the probability of eribulin being cost effective, the expected value of perfect information (EVPI, i.e. expected monetary loss caused by uncertainty), and the expected monetary loss (eML) caused by reimbursing a costineffective treatment. ${ }^{17}$ The risk associated with reimbursing eribulin equaled the EVPI plus the eML. ${ }^{17,18}$ These figures were scaled up to the number of eligible patients in the Netherlands, assuming 590 incident eligible patients (based on ${ }^{19}$ and assumptions ${ }^{20}$ ) The EVPI and eML were also expressed in QALYs. According to Dutch guidelines, a $€ 80,000$ per QALY willingness-to-pay threshold was used, corresponding to a burden of disease of $0.98 .^{21}$

The annual $\mathrm{BI}$ associated with reimbursing eribulin was calculated, assuming the uptake of eribulin observed in SONABRE after 2016 (47\%). The BI analysis compared the chemotherapy costs of eribulin versus the costs of non-eribulin chemotherapy only. A scenario analysis with a $100 \%$ uptake was performed. Supplemental material 2 , chapter 5 , provides a detailed explanation of the assumptions and calculations underlying the $\mathrm{BI}$ analysis.

Model inputs and outputs were face validated by a clinical expert. Model implementation model was verified by an external modeller and extreme value testing (supplemental material 2, Section 6, based on AdVISHE ${ }^{22}$ ).

\section{Results}

The PSA resulted in mean incremental QALY and costs of 0.07 and $€ 15,321$ respectively, leading to an ICER of $€ 220,608$ (Table 6.2, Figure 6.3). None of the scenario analyses resulted in an ICER below $€ 80,000$ per QALY (Table 6.2). At a $€ 80,000$ per QALY threshold, the probability of eribulin being cost effective was $1 \%$, the EVPI was $€ 13(<0.01 \mathrm{QALY})$, and the eML associated with reimbursing eribulin was $€ 9,765$ (0.12 QALY) per patient. This amounted to a total risk of €9,778 (0.12 QALY) per patient. For the Dutch $A B C$ population, these figures were $€ 7,681$ (0.1 QALY), €2.7 million (33.73 QALY) and €2.7 million (33.83 QALY) per year respectively (supplemental material 2, Section 4.5). A 85\% discount on the price of eribulin is required to meet the $€ 80,000$ per QALY threshold. The estimated annual $\mathrm{BI}$ of eribulin was $€ 1.9$ million, and $€ 4.1$ million when assuming a $100 \%$ uptake (supplemental material 2, Section 5). 


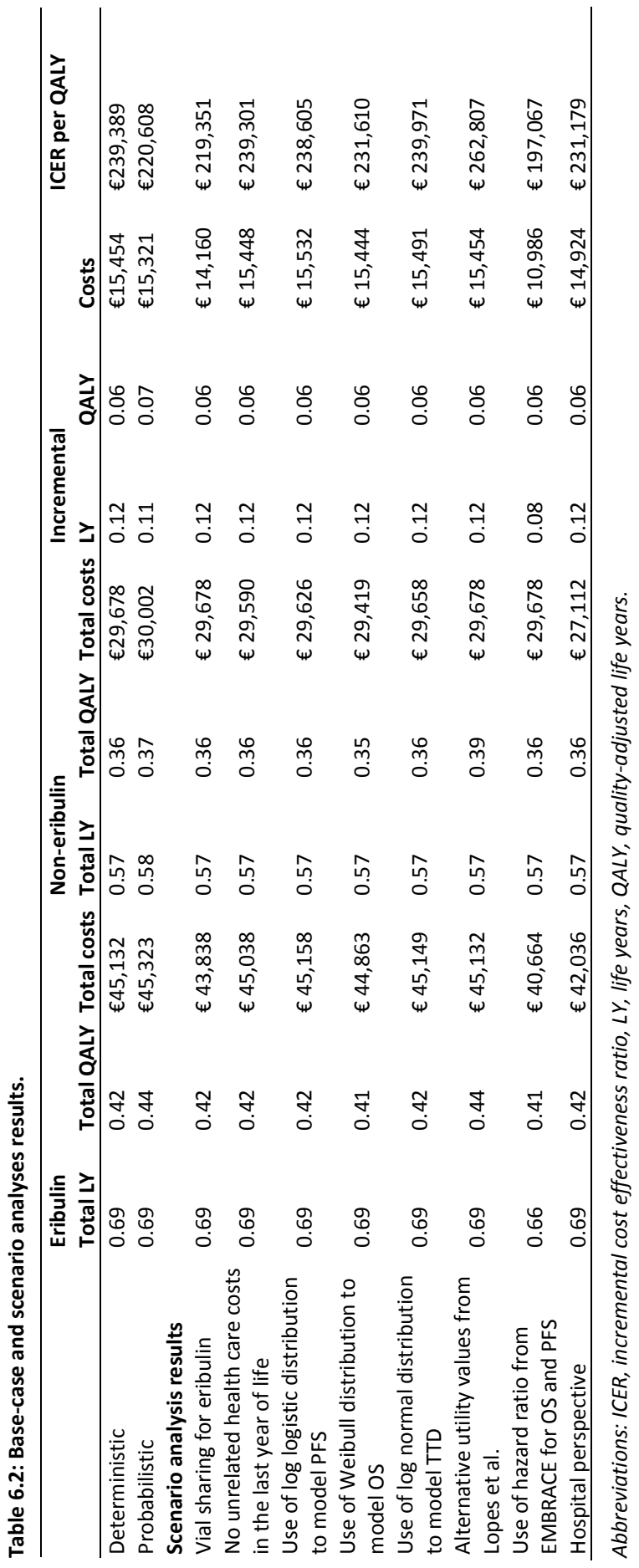




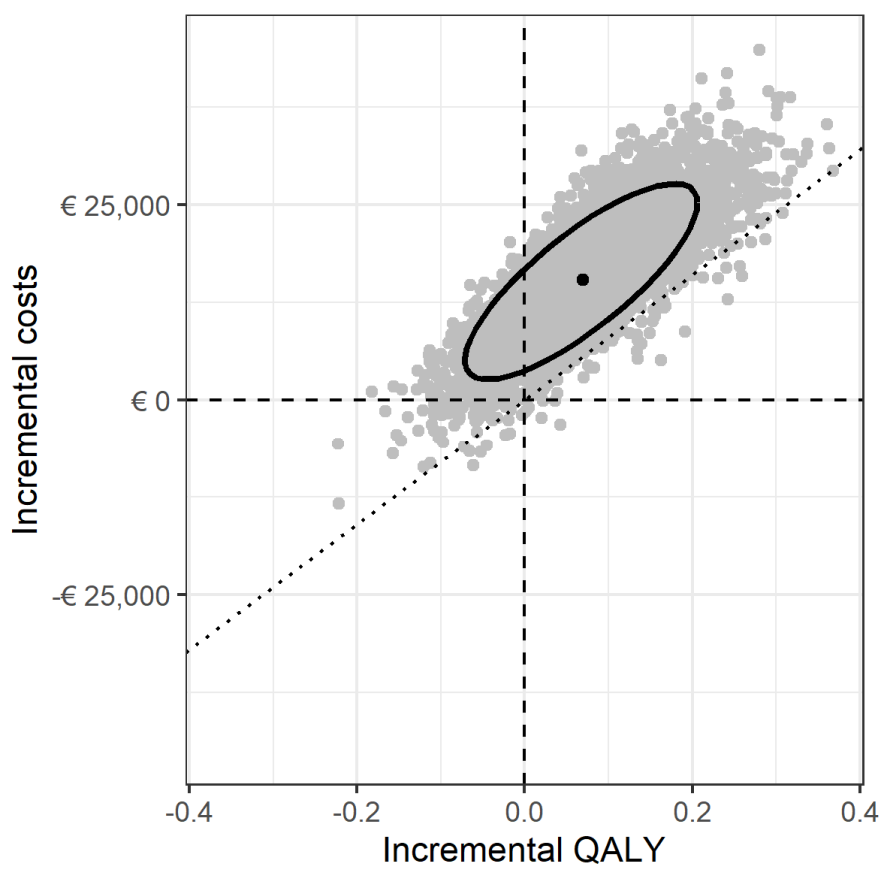

Figure 6.3: Incremental cost-effectiveness plane.

Legend: each dot represents the incremental results (costs and QALY) of each probabilistic iteration of the probabilistic sensitivity analysis. The dotted line represents the willingness-to-pay threshold of $€ 80,000$ per QALY. The ellipse represent the 95\% confidence interval of the incremental costs and QALY.

Abbreviations: $Q A L Y$, quality-adjusted life year.

\section{Discussion}

The current study investigated the cost effectiveness of eribulin versus non-eribulin chemotherapy as third or later chemotherapy line for $A B C$ patients. To the best of our knowledge, our analysis is the first cost-effectiveness analysis of eribulin using realworld data concerning the effectiveness, resource use and costs of $A B C$ patients. Additionally, quality of life data were obtained from patients treated in the same region. ${ }^{10}$ In this study, $67 \%$ of patients received eribulin as fourth or later chemotherapy. Eribulin provided higher health benefits (0.07 QALY) and costs $(€ 15,321)$ than non-eribulin chemotherapy, resulting in an ICER of $€ 220,608$ per QALY gained. The estimated risk associated with reimbursing eribulin was $€ 9,778$ per patient, and $€ 2.7$ million annually for the Dutch $A B C$ patient population. The estimated annual $\mathrm{BI}$ of eribulin was $€ 1.9$ million. 
Lopes et al. assessed the cost effectiveness of eribulin versus TPC as third (or later) chemotherapy line from a US payer perspective. Based on a model-based costeffectiveness analysis and using aggregate data from EMBRACE ${ }^{2}$, they concluded that eribulin was not cost effective (ICER: \$213,742 per QALY gained). ${ }^{16}$ Tremblay et al. compared eribulin with capecitabin and vinorelbin as second-line treatment from a South Korean health care perspective ${ }^{23}$, based on a partitioned survival using the Kaplan-Meier survival function of Study $301 .{ }^{24}$ This study, in which eribulin led to 0.24 incremental QALY and $\approx \$ 6,541$ incremental costs, concluded that eribulin was cost effective for patients with human epidermal growth factor receptor 2-negative tumours (ICER: 17 million ( $\approx$ 14,800) per QALY gained). ${ }^{23}$ Since eribulin may provide less benefits in more heavily pre-treated patients with more advanced diseases ${ }^{25}$, the extensive pre-treatment of our patients may explain the short OS in our study ${ }^{26}$ and partly the different ICERs between studies.

The current cost-effectiveness analysis has some limitations. Firstly, the number of patients who received eribulin and non-eribulin chemotherapy was small. Matching was used to reduce the potential bias due to confounding by indication; however, residual bias remained as we did not correct for (unmeasured) factors. Secondly, we partially implemented the societal perspective because costs estimates outside the hospital were unavailable (e.g. (un)paid work, informal care). Thirdly, our economic evaluation does not fully capture the value of having an additional, tolerable treatment option for $A B C$, which may support the added societal value of reimbursing eribulin. ${ }^{27}$ Our analysis may underestimate the life-cycle value of eribulin because it does not consider the potential market entry of a cheap generic of eribulin after its patent expiry. ${ }^{28,29}$ Since a potential generic should cost $15 \%$ of the price to be costeffective, the development of a cost-effective generic of eribulin is not likely.

Since January $1^{\text {st }} 2019$, only drugs with an annual BI over $€ 10$ million in the Netherlands require a formal cost-effectiveness assessment under the 'risk-oriented insurance package management' of ZIN. For eribulin, both the estimated $\mathrm{BI}$ and the risk associated with reimbursement are below this limit. One can however wonder whether $\mathrm{BI}$ appropriately represents risk, because it does not consider the opportunity costs incurred by reimbursing treatments, i.e. reimbursing eribulin leads to 33.83 sacrificed QALYs annually because money cannot be spent on other (costeffective) treatments. Additionally, (expensive) treatments will increasingly be indicated for small patient populations due to the increase in molecular diagnostics and personalised (cancer) treatment. ${ }^{30}$ Their individual $\mathrm{BI}$ and risk may remain under the ZIN's limit but, altogether, they may incur a risk on the health care system and lead to increased health care expenditure. ${ }^{31}$ These developments call for a redefinition of risk and more flexible reimbursement decisions to manage these 
risks. $^{30,32,33}$ Identifying the type and level of uncertainty surrounding a decision during (routinely performed) risk assessments ${ }^{17,34,35}$ and considering collected real-world evidence to optimise treatment use in practice are first steps to reach this goal.

In conclusion, using real-world data, eribulin is not cost effective as third or later chemotherapy line for extensively pretreated $A B C$ patients when considering its list price from a Dutch perspective. $\mathrm{BI}$ and risk incurred by reimbursing eribulin (respectively $€ 1.9$ and $€ 2.7$ million annually) were below the ZIN's risk limit. Although the individual risk incurred by new innovations may remain below the ZIN's limit, they require to be managed through flexible reimbursement schemes that are informed by trustworthy assessments of uncertainty and risk. 


\section{References}

1. College van Zorgverzekering. Eribulin (Halaven) bij lokaal gevorderde of gemetastaseerde borstkanker. 2013.

2. Cortes J, O'Shaughnessy J, Loesch D, et al. Eribulin monotherapy versus treatment of physician's choice in patients with metastatic breast cancer (EMBRACE): a phase 3 open-label randomised study. Lancet. 2011;377(9769):914-923.

3. Zorginstituut Nederland. Beoordeling dure specialistische geneesmiddelen [11/10/2019]. Available from: https://www.zorginstituutnederland.nl/over-ons/werkwijzen-en-procedures/adviseren-overen-verduidelijken-van-het-basispakket-aan-zorg/beoordeling-van-geneesmiddelen/beoordeling-durespecialistische-geneesmiddelen

4. Zorginstituut Nederland. Eribulin mesilaat (Halaven) bij $3 e$ en latere lijnsbehandeling gemetastaseerde borstkanker (wijziging beleid $t=4$ dossiers) 2016 [12/09/2019]. Available from: https://www.zorginstituutnederland.nl/publicaties/brief/2016/12/22/eribulin-mesilaat-halaven-bij3e-en-latere-lijnsbehandeling-gemetastaseerde-borstkanker-wijziging-beleid-t-4-dossiers

5. Pouwels XGLV, Geurts SME, Ramaekers BLT, et al. The relative effectiveness of eribulin for advanced breast cancer treatment: a study of the southeast Netherlands advanced breast cancer registry. Acta Oncologica. 2019:1-8.

6. Zorginstituut Nederland. Richtlijn voor het uitvoeren van economische evaluaties in de gezondheidszorg: Zorginstituut Nederland; 2015. Available from: https://www.zorginstituutnederland.nl/ over-ons/publicaties/publicatie/2016/02/29/richtlijn-voor-het-uitvoeren-van-economischeevaluaties-in-de-gezondheidszorg

7. Alarid-Escudero F, Krijkamp EM, Pechlivanoglou P, et al. A Need for Change! A Coding Framework for Improving Transparency in Decision Modeling. PharmacoEconomics. 2019;37(11):1329-1339.

8. R Core Team. R: A Language and Environment for Statistical Computing. Vienna, Austria: R Foundation for Statistical Computing; 2017.

9. Stuart EA. Matching methods for causal inference: A review and a look forward. Stat Sci. 2010; 25(1):1-21.

10. van Kampen RJW, Ramaekers BLT, Lobbezoo DJA, et al. Real-world and trial-based cost-effectiveness analysis of bevacizumab in HER2-negative metastatic breast cancer patients: a study of the Southeast Netherlands Breast Cancer Consortium. Eur J Cancer. 2017;79:238-246.

11. Dolan P, Gudex C, Kind P, et al. A social tariff for EuroQol: results from a UK general population survey; discussion paper no 138. Centre for Health Economics, University of York, York. 1995.

12. Lloyd A, Nafees B, Narewska J, et al. Health state utilities for metastatic breast cancer. Br J Cancer. 2006;95(6):683-690.

13. Zorginstituut Nederland. Medicijnkosten: Zorginstituut Nederland; 2017 [cited 2017 20-01-2017]. Available from: http://www.medicijnkosten.nl/

14. van Baal PH, Wong A, Slobbe LC, et al. Standardizing the inclusion of indirect medical costs in economic evaluations. Pharmacoeconomics. 2011;29(3):175-187.

15. Centraal Bureau voor de Statistiek. Consumer prices 2019 [23/09/2019]. Available from: https://opendata.cbs.nl/statline/\#/CBS/en/dataset/83131ENG/table?ts=1569221154525

16. Lopes G, Gluck S, Avancha K, et al. A cost effectiveness study of eribulin versus standard single-agent cytotoxic chemotherapy for women with previously treated metastatic breast cancer. Breast Cancer Res Treat. 2013;137(1):187-193.

17. Grimm SE, Strong M, Brennan A, et al. The HTA Risk Analysis Chart: Visualising the Need for and Potential Value of Managed Entry Agreements in Health Technology Assessment. Pharmacoeconomics. 2017;35(12):1287-1296.

18. Alarid-Escudero F, Enns EA, Kuntz KM, et al. "Time Traveling Is Just Too Dangerous" but Some Methods Are Worth Revisiting: The Advantages of Expected Loss Curves Over Cost-Effectiveness Acceptability Curves and Frontier. Value Health. 2019;22(5):611-618. 
19. Integraal kankercentrum Nederland. Breast cancer 2019 [12/11/2019]. Available from: https://www.iknl.nl/nkr-cijfers?fs\%7Cepidemiologie_id=7\&fs\%7Ctumor_id=369\&fs\%7Cregio_id= 135\&fs\%7Cgedrag_id=12\&fs\%7Cperiode_id =115\&fs\%7Cgeslacht_id=16\&fs\%7Cleeftijdsgroep_id=76\% 2C40\%2C41\%2C42\%2C43\%2C44\%2C45\&fs\%7Cjaren_na_diagnose_id=18\&fs\%7Ceenheid_id=2\&cs\%7 Ctype=column\&cs\%7CxAxis=leeftijdsgroep_id\&cs\%7Cseries=epidemiologie_id\&ts\%7CrowDimensions $=\& \mathrm{ts} \% 7 C$ columnDimensions=leeftijdsgroep_id\&lang\%7Clanguage=nl

20. Zorginstituut Nederland. Pakketadvies palbociclib (Ibrance ${ }^{\circledR}$ ) voor patiënten met lokaal gevorderde of gemetastaseerde borstkanker. 2017.

21. Versteegh MM, Ramos IC, Buyukkaramikli NC, et al. Severity-Adjusted Probability of Being Cost Effective [journal article]. PharmacoEconomics. 2019;37(9):1155-1163.

22. Vemer P, Corro Ramos I, van Voorn GA, et al. AdViSHE: A Validation-Assessment Tool of HealthEconomic Models for Decision Makers and Model Users. Pharmacoeconomics. 2016;34(4):349-361.

23. Tremblay G, Majethia U, Breeze JL, et al. Economic evaluation of eribulin as second-line treatment for metastatic breast cancer in South Korea. Clinicoecon Outcomes Res. 2016;8:485-493.

24. Kaufman PA, Awada A, Twelves $C$, et al. Phase III open-label randomized study of eribulin mesylate versus capecitabine in patients with locally advanced or metastatic breast cancer previously treated with an anthracycline and a taxane. J Clin Oncol. 2015;33(6):594-601.

25. Voutsadakis IA. A systematic review and pooled analysis of retrospective series of eribulin in metastatic breast cancer. Anticancer Drugs. 2017;28(5):557-564.

26. Cortes J, Twelves C. Impact of the number of prior chemotherapy regimens on outcomes for patients with metastatic breast cancer treated with eribulin: A post hoc pooled analysis. Breast J. 2020; 26(7):1347-1351

27. Drummond $\mathrm{M}$, Tarricone $\mathrm{R}$, Torbica $\mathrm{A}$. Assessing the added value of health technologies: reconciling different perspectives. Value Health. 2013;16(1 Suppl):S7-S13.

28. Grimm SE, Dixon S, Stevens JW. When Future Change Matters: Modeling Future Price and Diffusion in Health Technology Assessments of Medical Devices. Value Health. 2016;19(6):720-726.

29. Hoyle M. Accounting for the drug life cycle and future drug prices in cost-effectiveness analysis. PharmacoEconomics. 2011;29(1):1-15.

30. Mincarone P, Leo CG, Sabina S, et al. Reimbursed Price of Orphan Drugs: Current Strategies and Potential Improvements. Public Health Genomics. 2017;20(1):1-8.

31. Birch S, Gafni A. The biggest bang for the buck or bigger bucks for the bang: the fallacy of the costeffectiveness threshold. J Health Serv Res Policy. 2006;11(1):46-51.

32. Trosman JR, Weldon CB, Gradishar WJ, et al. From the Past to the Present: Insurer Coverage Frameworks for Next-Generation Tumor Sequencing. Value Health. 2018;21(9):1062-1068.

33. Vella Bonanno P, Ermisch M, Godman B, et al. Adaptive Pathways: Possible Next Steps for Payers in Preparation for Their Potential Implementation [Review]. Frontiers in Pharmacology. 2017;8:497.

34. Grimm SE, Pouwels X, Ramaekers BLT, et al. Development and Validation of the TRansparent Uncertainty ASsessmenT (TRUST) Tool for Assessing Uncertainties in Health Economic Decision Models [journal article]. PharmacoEconomics. 2020;38(2):205-216.

35. Zorginstituut Nederland. Procedure beoordeling extramurale geneesmiddelen wordt aangepast 2018 [13/11/2019]. Available from: https://www.zorginstituutnederland.nl/actueel/ nieuws/2018/11/21/ procedure-beoordeling-extramurale-geneesmiddelen-wordt-aangepast 


\section{Appendices}

\section{Supplemental material 1 to An economic evaluation of eribulin for advanced breast cancer treatment based on the SOutheast Netherlands Advanced BREast cancer (SONABRE) Registry}

\section{Matching procedure}

For the matching procedure, Genetic Matching was applied on treatment lines of eligible patients. This meant that the treatment line wherein patients received eribulin (eribulin group) were matched to treatment of patients in the non-eribulin group based on the age of patients at the start of the treatment line, tumour subtype, the number of previous chemotherapy received before the treatment line, and the presence of central nervous metastasis. Since the non-eribulin group was selected based on the eribulin eligibility criteria, all treatment lines fulfilled the inclusion criteria. Compared to the unmatched non-eribulin group, Genetic Matching on the treatment lines increased the similarity in baseline characteristics between the eribulin and non-eribulin groups. Details on the matching procedure and patient characteristics of each group are provided elsewhere. ${ }^{1}$

\section{Selection survival curves (overall survival, progression-free survival, and time to treatment discontinuation)}

Survival curves for the base-case analysis were selected based on the selection algorithm described in the NICE DSU TSD 14. Firstly, we investigated whether the proportional assumption between eribulin and non-eribulin chemotherapies held, based on visual inspection of the log-cumulative hazard plots. We therefore decided to fit stratified (i.e. independent) survival models to each treatment group. Secondly, we investigated whether monotonic hazard models should be used and concluded it should not since smoothed hazard plots were not straight lines (data not shown). The Weibull model was thus not considered for OS, even though it had one of the lowest Akaike Information Criterion (AIC). Thirdly, we the assessed the statistical fit of different distributions to the data (Table S6.1) based on the AIC (the lowest the AIC, the best the statistical fit, also in case of negative values). We then presented the survival probability estimated by the three best fitting curves to a clinical expert $(\mathrm{MdB})$, which informed the final curve selection. The clinical expert was asked which of the presented curves provided the most plausible extrapolation for each outcomes. 
The selected curves were: gamma for OS, log normal for PFS, and log-logistic for TTD (provided in Figure 6.2 of the Manuscript).

Table S6.1: Statistical fit statistics (Akaike Information Criterion) of survival models fitted to the overall survival, progression-free survival, and time to treatment discontinuation data.

\begin{tabular}{lccc}
\hline Distribution & OS & PFS & TTD \\
\hline Exponential & 90.064 & 13.323 & -15.937 \\
Weibull & 79.899 & 12.903 & -19.196 \\
Gompterz & 84.542 & 16.769 & -14.237 \\
Lognormal & 82.132 & 7.198 & -32.411 \\
Loglogistic & 80.657 & 6.654 & -34.764 \\
Gamma & 79.041 & 10.854 & -23.552 \\
Generalised gamma & 82.854 & 10.08 & -29.757 \\
\hline
\end{tabular}

\section{Analysis of resource use data from SONABRE}

\section{Systemic treatment costs}

For systemic treatment costs administered during PFS, missing doses ( $N=16,3 \%$ ) of eribulin were imputed using single stochastic imputation in order to be able to implement the Dirichlet distribution. The missing doses of non-eribulin chemotherapy were imputed using multiple imputation (five data sets, predictive mean matching). Pooling was performed using the pool.scalar function. Table S6.2 provides a detailed overview of the inputs to calculate chemotherapy costs of the matched non-eribulin group. 


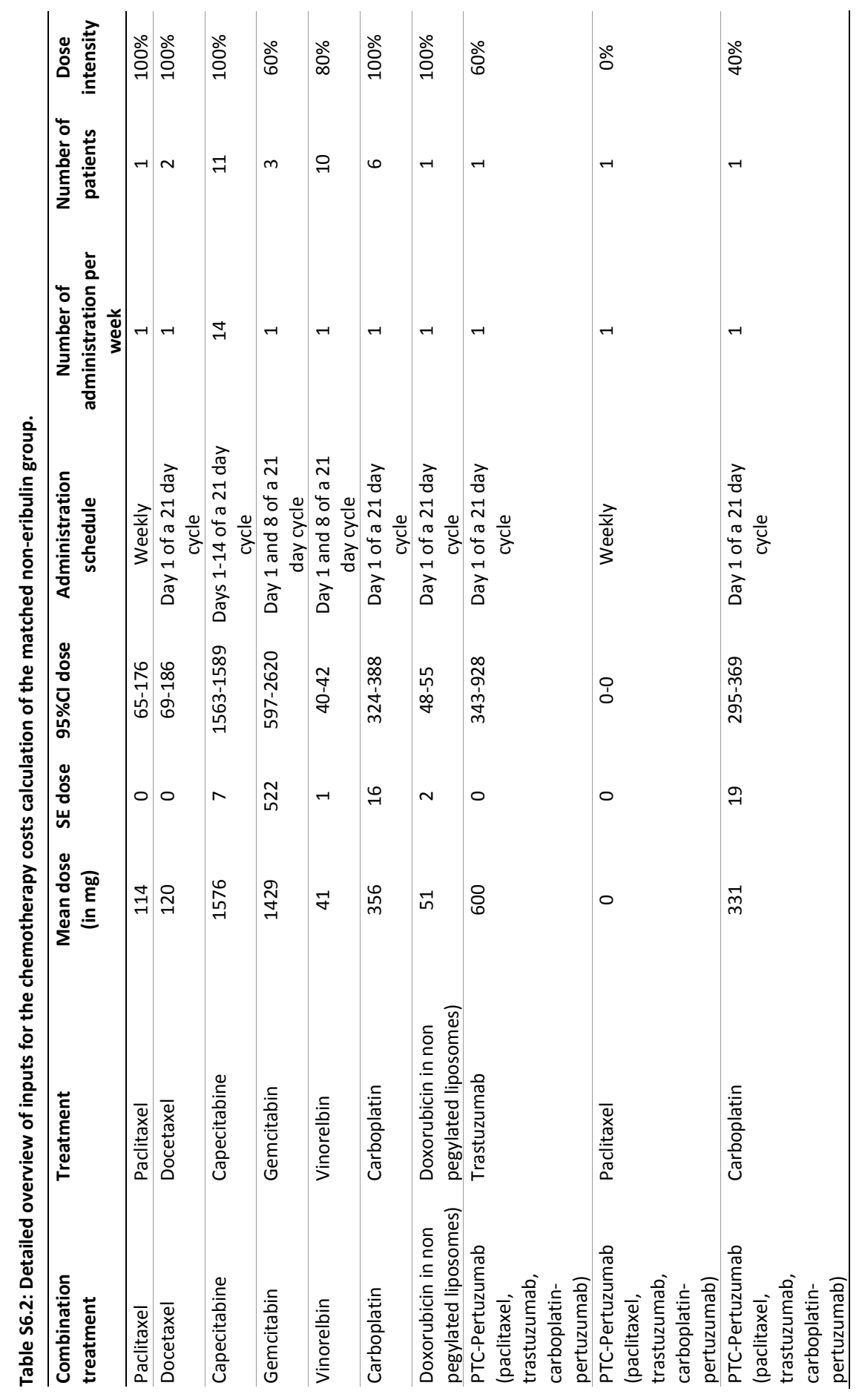




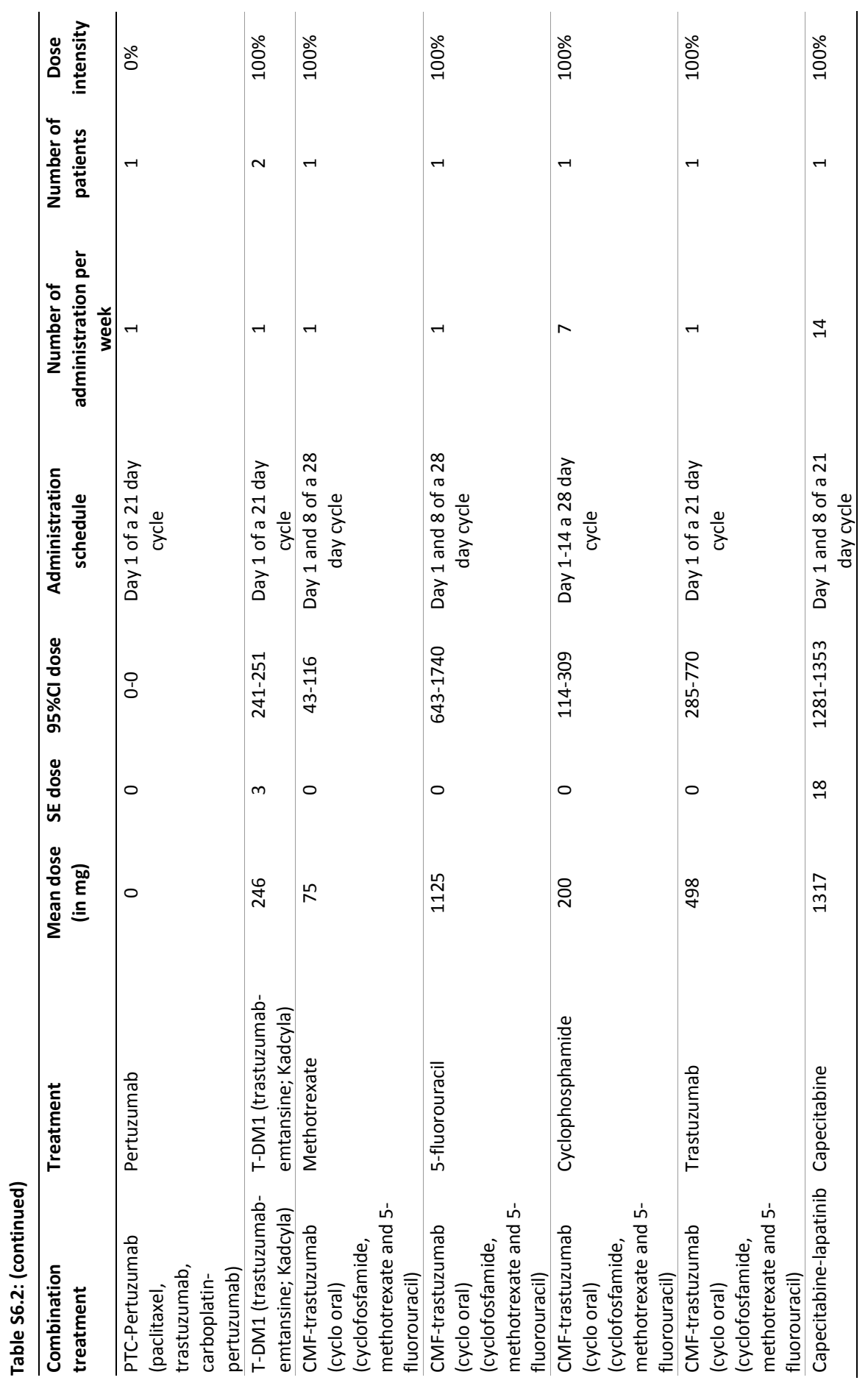




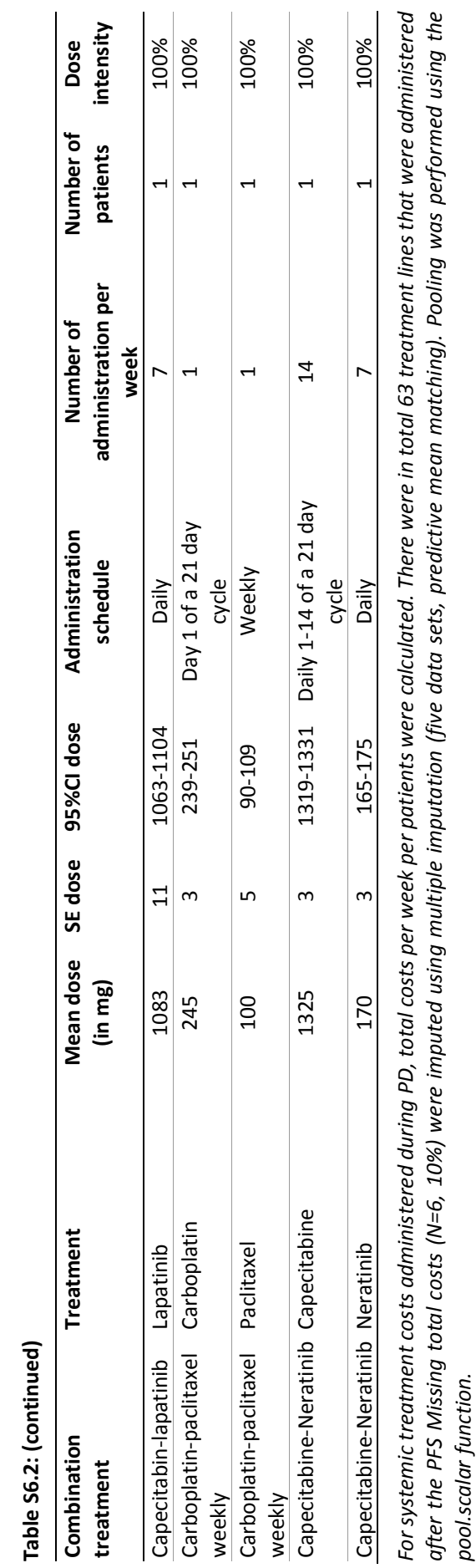


Monitoring, radiotherapy, and surgery costs in the progression-free and progressed disease health states

In SONABRE, resources consumed during systemic treatments are registered in treatment lines. The mean and standard errors of monitoring, radiotherapy, surgery were calculated as follows. Firstly, all treatment lines administered to eligible patients after the first of July 2013 were identified. This amounted 187 treatment lines, and no distinction was made between treatment lines administered during PFS and PD. The second step consisted of calculating total costs per treatment lines for all treatment lines. After that, multiple imputation (five data sets) using predictive mean matching was used to impute missing values (R mice package). In each data sets, the mean costs per week were calculated for the treatment line administered during PFS. The total length of PD and the associated costs were calculated by summing up the length and costs associated with treatment lines administered after the PFS treatment line. Mean costs and standard errors based on the five imputed data sets were calculated using the pool.scalar function.

\section{Hospitalisation costs}

Total hospitalisation costs before and after the progression date were aggregated in order to obtain hospitalisation costs for the PF and PD health states, respectively. There were 147 (45 eribulin, 102 unmatched non-eribulin) treatment lines that were included in the calculation of the PF hospitalisation costs, of those 22 (15\%) had missing values. For the PD hospitalisation costs, there were 54 PF treatment lines that were followed by subsequent treatment lines. Of these, 6 had missing values (11\%). Missing values were imputed using predictive mean matching (five data sets) and pooled using the pool.scalar function.

\section{Travel cost calculation}

Travel costs of a visit were calculated by multiplying the mean distance to the hospital $(7 \mathrm{~km})$ by the costs per kilometer (€0.19) (for a return journey). Mean parking costs (€3) were added to the travel costs. These estimates were obtained from the Dutch guidelines for health economic evaluation in health care. ${ }^{2}$

The calculation of the travel costs for one visit are thus: $7 * 0.19 * 2+3=€ 5.66$. 
Inputs used for the Dirichlet distribution of eribulin

Table S6.3 provides an overview of how often each combination of vials were administered to patients. This distribution was used to calculate the costs of one administration of eribulin, considering vial waste.

Table S6.3: Distribution of administered eribulin vials

\begin{tabular}{lcc}
\hline Types of vial & Number of administration & Price per type of vial \\
\hline No administration & 17 & 0.00 \\
1 vial of 3mg & 11 & 624.17 \\
1 vial of 3mg \& 1 vial of 2mg & 360 & 1040.29 \\
2 vials of 3mg & 80 & 1248.34 \\
\hline
\end{tabular}

\section{Prices}

Tables S6.4 and S6.5 provides an overview of the prices used for the different resources used. Table 4 focuses on the imaging and blood transfusions resources used. Table S6.5 provides an overview of the price per $\mathrm{mg}$ of each treatment. This price per $\mathrm{mg}$ has been multiplied by the observed dose administered to patients included in SONABRE (registered per $\mathrm{mg}$ ).

Table S6.4: Unit prices of resources use included in the monitoring (scans, blood tests, visits), surgery and hospital costs.

\begin{tabular}{lcc}
\hline Type of resource & Unit price & Sources \\
\hline Ascites drainage & $€ 143$ & {$[3]$} \\
CT scan abdomen & $€ 192$ & {$[3]$} \\
CT scan thorax \& abdomen & $€ 378$ & {$[3]$} \\
CT scan thorax & $€ 185$ & {$[3]$} \\
CT scans (general) & $€ 132$ & {$[3]$} \\
DEXA scans & $€ 79$ & {$[3]$} \\
Heart ultrasounds & $€ 79$ & {$[3]$} \\
Echography & $€ 89$ & {$[3]$} \\
MRI scan & $€ 258$ & {$[3]$} \\
MUGA scan & $€ 79$ & {$[3]$} \\
Pleura drainage & $€ 143$ & {$[3]$} \\
Bones scan & $€ 143$ & {$[3]$} \\
PET-MRI scan & $€ 1,396$ & {$[3]$} \\
PET-CT scan normal dose & $€ 1,515$ & {$[3]$} \\
CA15.3 measurement & & Internal \\
PET-CT scan low dose & & Internal \\
X-ray & & Internal \\
Abdominal surgery & & Internal \\
Ablatio of the breast & & Internal \\
Axillary lymph node dissection & & Internal \\
Central venous catheter & & Internal \\
Liver surgery & & Internal \\
Neurosurgery of the brain & & Internal \\
\hline
\end{tabular}


Table S6.4: (continued)

\begin{tabular}{lcc}
\hline Type of resource & Unit price & Sources \\
\hline Orthopedic surgery of extremities or pelvis & & Internal \\
Orthopedic surgery or neurosurgery of the spine & & Internal \\
PICC catheter & & Internal \\
Port-a-cath & & Internal \\
Pulmonary surgery & & Internal \\
Resection of metastasis on other location & & Internal \\
Resection of skin metastasis & & Internal \\
Stent bile duct & & Internal \\
Stent gastro-intestinal tract & & Internal \\
Surgery contralateral axilla & & Internal \\
Surgery of nodes (outside axilla) & & Internal \\
Sentinel node procedure & & {$[3]$} \\
Erythrocytes transfusion & $€ 220$ & {$[3]$} \\
Thrombocytes transfusion & $€ 531$ & {$[3]$} \\
Outpatient visit & $€ 134$ & {$[3]$} \\
Emergency visit & $€ 263$ & {$[3]$} \\
Telephone consultation & $€ 17$ & {$[3]$} \\
One day at the intensive care unit & $€ 1,206$ & {$[3]$} \\
One day of inpatient care & $€ 647$ & Internal \\
Ablatio with direct reconstruction with artificial material & & Internal \\
Hickmann & & Internal \\
Lumpectomy & & {$[4]$} \\
Radiotherapy fraction (any site) & $€ 253$ & Internal \\
Secundary reconstruction with artificial material & & \\
\hline
\end{tabular}


Table S6.5: Price per mg of $s$ used to calculated costs in the model.

\begin{tabular}{|c|c|c|}
\hline Type of resource & Price per $\mathrm{mg}$ & Sources \\
\hline 5-fluorouracil & $€<0.01$ & [5] \\
\hline Alendronic acid (fosamax) & $€ 0.01$ & [5] \\
\hline Anastrozol (arimidex) & $€ 0.04$ & [5] \\
\hline Bevacizumab & $€ 3.34$ & [5] \\
\hline Brivanib & $€ 1.00$ & [5] \\
\hline Caelyx (doxorubicin in non-pegylated lyposomes) & $€ 22.00$ & {$[5]$} \\
\hline Capecitabin & $€<0.01$ & [5] \\
\hline Carboplatin & $€ 0.33$ & [5] \\
\hline Cyclophosphamide & $€ 0.02$ & [5] \\
\hline Cytarabine & $€ 0.03$ & [5] \\
\hline Docetaxel & $€ 5.72$ & [5] \\
\hline Doxorubicin (adriamycin) & $€ 22.07$ & [5] \\
\hline Epirubicine (4-epi-adriamycin) & $€ 1.84$ & [5] \\
\hline Eribulin & $€ 472.86$ & [5] \\
\hline Etoposide & $€ 0.18$ & [5] \\
\hline Everolimus & $€ 12.28$ & [5] \\
\hline Exemestane (aromasin) & $€<0.01$ & [5] \\
\hline FEC100 (5-fluorouracil, epirubicin and cyclophosphamide) & $€ 1,011$ & [5] \\
\hline Fulvestrant (faslodex) & $€ 1.14$ & [5] \\
\hline Gemcitabin & $€ 0.10$ & [5] \\
\hline Ibandronic acid (bonviva, bondronat) & $€ 0.11$ & [5] \\
\hline Lapatinib & $€ 0.07$ & [5] \\
\hline Letrozole (femara) & $€ 0.02$ & [5] \\
\hline Medroxyprogesteron ((depo)-provera)) & $€<0.01$ & [5] \\
\hline Megestrol (megace) & $€ 0.02$ & [5] \\
\hline Methotrexate & $€ 0.23$ & [5] \\
\hline Mitomycin & $€ 3.25$ & [5] \\
\hline Myocet (doxorubicin in non-pegylated lyposomes) & $€ 22.07$ & [5] \\
\hline Olaparib & $€ 0.32$ & {$[5]$} \\
\hline Paclitaxel & $€ 2.28$ & [5] \\
\hline Palbociclib & $€ 1.17$ & [5] \\
\hline Pertuzumab & $€ 6.13$ & [5] \\
\hline T-DM1 (trastuzumab-emtansine; Kadcyla) & $€ 19.18$ & [5] \\
\hline Tamoxifen & $€ 0.01$ & [5] \\
\hline Trastuzumab & $€ 3.64$ & {$[5]$} \\
\hline Vinorelbin & $€ 2.44$ & [5] \\
\hline
\end{tabular}




\section{CHEERS statement}

- item 1, title: ok

- item 2, abstract: ok

- item 3, context + RQ: ok

- $\quad$ item 4, target pop: ok

- item 5, setting and location: ok

- item 6, perspective: ok

- $\quad$ item 7, comp: ok

- item 8, time horizon: ok

- item 9, discount rate: ok

- item 10, choice health outcome: ok

- item 11, measurement effectiveness: ok

- item 12, measurement and valuation preferences: ok

- item 13, estimating resource and costs: ok

- item 14, currency, price date, and conversion: ok

- item 15, model: ok

- item 16, assumptions: ok

- item 17, analytical methods: ok

- item 18, study parameters: ok

- item 19, incremental costs and outcomes: ok

- item 20, characterising uncertainty: ok

- item 21, heterogeneity: not applicable

- item 22, study findings, limitations, generalisability and current knowledge: ok

- item 23, funding: ok

- $\quad$ item 24, COI: ok 


\section{References}

1. Pouwels XGLV, Geurts SME, Ramaekers BLT, et al. The relative effectiveness of eribulin for advanced breast cancer treatment: a study of the southeast Netherlands advanced breast cancer registry. Acta Oncologica. 2019:1-8.

2. Zorginstituut Nederland. Richtlijn voor het uitvoeren van economische evaluaties in de gezondheidszorg: Zorginstituut Nederland; 2015. Available from: https://www.zorginstituutnederland.nl/ over-ons/publicaties/publicatie/2016/02/29/richtlijn-voor-het-uitvoeren-van-economischeevaluaties-in-de-gezondheidszorg

3. Hakkaart-van Roijen L, Van der Linden N, Bouwmans C, et al. Kostenhandleiding. Methodologie van kostenonderzoek en referentieprijzen voor economische evaluaties in de gezondheidszorg. Geactualiseerde versie 2015. In: Nederland. Z, editor. 2015.

4. Ramaekers $\mathrm{BL}$, Joore MA, Lueza $\mathrm{B}$, et al. Cost effectiveness of modified fractionation radiotherapy versus conventional radiotherapy for unresected non-small-cell lung cancer patients. J Thorac Oncol. 2013;8(10):1295-1307.

5. Zorginstituut Nederland. Medicijnkosten: Zorginstituut Nederland; 2017 [cited 2017 20-01-2017]. Available from: http://www.medicijnkosten.nl/ 


\section{Supplemental material 2}

The supplementary material 2 is an executable version of the cost-effectiveness model which is available online: https://doi.org/10.1080/0284186X.2020.1775289 


\section{Chapter 7}

\section{Increasing the transparency and accessibility of survival analysis for decision analytic models: the PERSUADE template}

Bram L.T. Ramaekers, Xavier G.L.V. Pouwels, Sabine E. Grimm, Manuela A. Joore Manuscript in preparation for submission 


\section{Chapter 8}

\section{Assessing survival model selection uncertainty in cost-effectiveness analyses}

Xavier G.L.V. Pouwels, Bram L.T. Ramaekers, Sandra M.E. Geurts, Vivianne C.G. Tjan-Heijnen, Maaike de Boer, Manuela A. Joore 


\section{Chapter 9}

\section{Development and validation of the}

TRansparent Uncertainty ASsessmenT (TRUST) tool for assessing

uncertainties in health economic decision models 


\section{Abstract}

\section{Background}

An increased number of technologies obtaining marketing authorisation based on sparse evidence causes growing uncertainty and risk within health technology reimbursement decision-making. To ensure that uncertainty is considered and addressed within HTA recommendations, uncertainties need to be identified, included in health economic models and reported.

\section{Objective}

To develop the TRansparent Uncertainty ASsessmenT (TRUST) tool for systematically identifying, assessing and reporting uncertainties in decision models, with the aim of making uncertainties and their impact on cost-effectiveness more explicit and transparent.

\section{Methods}

TRUST was developed by drawing on the uncertainty and risk assessment literature. For its development and validation, we performed HTA stakeholder discussion meetings and interviews, and applied it in six real-world HTA case studies in the Netherlands and the United Kingdom.

\section{Results}

The TRUST tool enables identifying and categorising uncertainty according to sources of uncertainty (transparency issues, methodology issues, and issues with evidence: imprecision, bias \& indirectness and unavailability) in each model aspect. The source of uncertainty determines the appropriate analysis. The impact of uncertainties on cost-effectiveness is also assessed. Stakeholders considered using the tool to be feasible and of value for transparent uncertainty assessment. TRUST can be used during model development and / or model review.

\section{Conclusion}

The TRUST tool enables systematic identification, assessment and reporting of uncertainties in health economic models and may contribute to more informed and transparent decisionmaking in the face of uncertainty. 


\section{Introduction}

Uncertainty is rife in health technology assessments (HTA) leading to increased complexity in decision-making. Drug approval processes are typically based on evidence that exhibits several limitations. ${ }^{1-3}$ These may include, amongst others, a lack of data on a certain relevant health outcome, such as long-term treatment effectiveness, limited generalisability of evidence to the decision context, and small sample sizes as well as data immaturity. The result is that health economic models used to inform such decisions (further referred to as 'models') suffer from uncertainty in for instance their inputs, extrapolations, and / or assumptions. ${ }^{4,5}$ This uncertainty about (cost-)effectiveness of new health technologies means HTA decision-making is cognitively challenging. ${ }^{2}$ Things are getting worse: recent pressures to grant market access earlier in the drug development process result in decisions having to be made based on increasingly immature evidence ${ }^{6-9}$, or on single-arm studies. ${ }^{10,11}$ If these uncertainties are not fully appreciated, decisions may be based on a biased view of expected incremental net benefit and the associated uncertainty. This in turn would lead to an increased risk of making a suboptimal decision, which can be described as opportunity losses, or potential losses in population health that could be averted with gathering additional evidence. ${ }^{12}$

Unfortunately, uncertainties are often not fully characterised in models. ${ }^{5}$ We think that this happens for two reasons: there is a) an undue emphasis on imprecision issues, and b) difficulty in precisely expressing all uncertainty. With respect to a), this undue emphasis means that uncertainty about quality and generalisability of evidence to the setting in question is not always considered. ${ }^{13}$ This is an omission that has long been recognised in the assessment of quality of evidence ${ }^{14}$ and the risk assessment literature. ${ }^{15,16}$ The Grading of Recommendations Assessment, Development and Evaluation (GRADE) working group, for instance, highlights the lack of focus on the quality of clinical evidence and proposes a system to acknowledge and even rate it. $^{14,17}$ To the best of our knowledge, however, a tool for systematically identifying uncertainty about the quality of evidence used in models is currently lacking. Regarding b), uncertainty is most completely characterised with a full explicit probability distribution. ${ }^{4,5,18,19}$ In theory it may be possible to represent all uncertainty in this way, for example using methods to express expert opinion ${ }^{20,21}$ and / or structural uncertainty. ${ }^{22,23,24}$ In practice, tight HTA timelines and resource constraints may be prohibitive. Other barriers include that people may not be able to express their beliefs in the form of probabilities, ${ }^{25}$ or that the distribution of the additional structural uncertainty parameter may be difficult to specify. ${ }^{13}$ Therefore, most models 
do not incorporate all uncertainty in a quantitative manner and thus do not fully express the risk associated with a decision in a numerical value. ${ }^{13,26}$

In the worst case, the lack of systematic identification and assessment of all uncertainties present in models may result in a state of uncertainty ignorance, leaving decision-makers unaware of the risk associated with a decision. But even where uncertainty information is present, it is usually scattered throughout reports, and decision-makers are left with the cognitively challenging task of identifying all uncertainties and translating pieces of qualitative and quantitative uncertainty information into an overall assessment of uncertainty and risk. ${ }^{15}$ A unified, comprehensive framework for identifying uncertainties in models, assessing their impact and reporting the results is therefore needed.

In this paper, we develop a tool for systematically identifying, assessing and reporting uncertainty in health economic submissions. The paper is structured as follows: in the next section, we describe the development and validation of the TRansparent Uncertainty ASsessmenT (TRUST) tool. In the Results section, we present findings from interviews and case study and the final tool, as well as its place in the reimbursement decision-making process. We conclude with a discussion and conclusion.

\section{Development of TRUST}

\subsection{The theoretical framework}

We developed a first version of the tool based on the literature on uncertainty and risk frameworks in HTA and risk assessment disciplines. ${ }^{14,16,18,27-29}$ Relevant literature was identified based on a review of the literature on uncertainty frameworks. We used a pragmatic approach to reviewing the literature and started with a citation search of a paper known to us by van Asselt and Rotmans (2002). ${ }^{16}$ We furthermore used a recently published interdisciplinary literature review of uncertainty frameworks by van der Bles et al $(2019)^{18}$ and checked its references for relevant uncertainty literature. Concepts surrounding uncertainty from the identified literature were checked for applicability and relevance in the HTA decision-making context by a group of researchers that drew upon experience in HTA decision-making in the Netherlands and in the United Kingdom (UK). MJ, JG, BR, SG, and XP have been active members of a UK's National Institute for Health and Care Excellence (NICE) Evidence Review Group and have built models that informed reimbursement decision-making. 
SK has been a senior policy advisor of the Dutch Health Care Institute (ZIN) since 2012 and MJ a member of the scientific advisory committee of ZIN since 2013.

\subsection{Model aspects}

To systematically identify and assess uncertainty in models, it was necessary to establish the locations where uncertainties could occur. A publication by Walker et al. (2003) developed a "conceptual basis for the systematic treatment of uncertainty in model-based decision support activities such as policy analysis, integrated assessment and risk assessment" ${ }^{27}$ The authors identified the following locations: context (including natural, technological, economic, social and political, representation), model (structure and technical), and inputs (driving forces, system data), parameters, model outcomes. Standard HTA dossier templates from UK and Netherlands were used to inform more detailed descriptions of model aspects.

\subsection{Source of uncertainty}

Different uncertainty frameworks provide different taxonomies of uncertainty and they use different terminology to describe uncertainty. Walker et al. (2003) consider that uncertainty has to be viewed as a three-dimensional concept: ${ }^{27}$ its nature (whether it is epistemic or aleatory), its level (from deterministic knowledge to ignorance), and its locations (what is affected in the model-based assessment). Epistemic uncertainty is that which could be reduced with further knowledge, aleatory uncertainty is caused by inherent variability. ${ }^{18}$ In our paper, much like in the related health policy decision-making literature, ${ }^{28}$ we focus on epistemic uncertainty, i.e. that which can be addressed in some form. The "levels" in the Walker et al framework range from deterministic knowledge via statistical uncertainty (precision), scenario uncertainty (where the mechanism leading to different possible outcomes is not well understood) and recognised ignorance (scientific evidence for developing scenarios is weak), to total ignorance (unknown unknowns). ${ }^{27}$ These different levels were defined with the intention to call for different actions to address them. It is worth noting that this list of levels is a pragmatic summary of the seven "sources" of epistemic uncertainty identified in a previous publication (van Asselt \& Rotmans, 2002). ${ }^{16}$ The Walker et al framework has been adapted for the Numeral, Unit, Spread, Assessment and Pedigree (NUSAP) approach for uncertainty assessment, ${ }^{29}$ which added methodological unreliability as a further type of uncertainty.

The GRADE working group outlined a broader framework for assessing quality of evidence. ${ }^{17}$ The group established five limitations that can lead to downgrading of the quality of evidence: study limitations resulting in bias, inconsistent results (across 
studies), indirectness of evidence, imprecision and publication bias. ${ }^{14,17}$ Van der Bles et al. (2019) distinguished between "levels" of direct and indirect uncertainty ${ }^{18}$, where direct uncertainty pertains to imprecision in estimates which can be communicated in quantitative terms, and indirect uncertainty to the quality of the underlying knowledge, which will typically be communicated as a list of caveats (GRADE was named as an example tool that facilitates assessing indirect uncertainty). The ISPORSMDM taskforce report ${ }^{28}$ makes a distinction between methodological uncertainty, parameter uncertainty, stochastic uncertainty, heterogeneity, and structural uncertainty.

Our final selection of possible sources of uncertainty was informed by these taxonomies and based on the following consideration: as different approaches are used in practice to manage uncertainty, it should be possible to match sources of uncertainty relevant to decision-makers with an approach to dealing with uncertainty. $^{27}$

\subsection{Expert feedack}

The TRUST tool was based on the theoretical framework described above and further developed based on several rounds of feedback from HTA experts. After the theoretical framework was established, initial feedback was obtained by means of a discussion meeting with advisors of ZIN. The revised TRUST tool was presented at academic conferences (EUHEA 2018 Maastricht and IHEA 2019 Basel) as well as at further discussion meetings at ZIN, Maastricht University Medical Center and Radboud University Medical Center. TRUST was presented during a HTA committee meeting for tomosynthesis for breast cancer population screening in the Netherlands. Individual in-depth semi-structured interviews with different international stakeholders $(\mathrm{N}=11)$ were performed to finalise the content of the tool, validate it and obtain insights into barriers to its use, user-friendliness and feasibility. These stakeholders were chosen to represent the following stakeholder groups: HTA policy advisors $(n=2)$, independent academic advisors for HTAs $(n=4)$, an appraisal committee member $(n=1)$, and industry stakeholders ( $n=4$, one working in a pharmaceutical company and 3 for HTA consultancies) and convenience sampling was used to establish contact. The interviews were recorded, transcribed and analysed by themes. The interview guide can be found in Appendix 1. Interviews were used to inform the selection of model aspects and sources of uncertainty to include in TRUST, terminology, and to highlight potential feasibility and usability issues. 


\subsection{Case studies}

The tool was tested for its validity and whether it "worked" in practice by means of application in five retrospective HTA case studies on expensive medicines and one ongoing HTA, the latter being on tomosynthesis for breast cancer population screening in the Netherlands. Four of the retrospective case studies were for ZIN in the Netherlands, and these were performed by two researchers independently, who then compared notes to identify challenges in the use of TRUST and potential interrater variability. The fifth retrospective case study was a NICE appraisal (Pembrolizumab for treating relapsed or refractory classical Hodgkin lymphoma) and this is presented here for illustration purposes. This application of TRUST was based on the information available in the article, ERG report and company submission. ${ }^{11}$ The case studies were selected based on convenience sampling: the ZIN referred us to the four retrospective Dutch case studies, and both the Dutch prospective study and the NICE study were projects in which members of our research team were involved.

\section{Results}

\subsection{Findings from stakeholder interviews and case studies}

Insights from stakeholder interviews were an important part of the development of this tool. The most important findings include (see Box 9.1 for more detail):

1. Keep it concise and simple

2. Rating uncertainty with a score may create a false sense of validity and be timeconsuming

3. Definitions and descriptions need to be clear

4. Time as the main barrier to the use of TRUST

The selected case studies represented a range of potential uncertainty issues encountered in health economic models. They included appraisals of one screening technology (Tomosynthesis for breast cancer population screening in the Netherlands), and five drugs: Pembrolizumab for treating relapsed or refractory classical Hodgkin lymphoma in the NHS England \& Wales, and four in the Netherlands, namely: lumacaftor/ivacaftor compared to best supportive care for treatment of cystic fibrosis in patients aged 12 years and older who are homozygous for the F508del mutation in the CFTR gene, nivolumab for treatment of patients with nonsmall cell lung cancer, rifaximine compared to treatment with lactulose alone for the treatment of recurrent episodes of manifest hepatic encephalopathy in patients aged 
18 and over, and eculizumab for the treatment of patients with atypical hemolytic uremic syndrome.

Box 9.1: Interview and case study findings.

\section{Keep it concise and simple}

Although there were conflicting view points on this (some respondents wished to add more response options or additional detail), the general feedback was that usability and interpretation of findings would improve with a version as concise as possible. The TRUST tool was simplified, for example by having dichotomous questions in the identification of sources of uncertainty in each model aspect, rather than providing three or four level response options. A summary was developed, which only shows the main issues at one glance (Figure 9.1 and Appendix 9.2).

2. Rating uncertainty with a score may create a false sense of validity and be time-consuming

The first version of TRUST included a scoring system that was inspired by GRADE. This was intended to help assess, based on the different sources of uncertainty, the severity of the overall uncertainty associated with one model aspect. However, this caused multiple issues: there was significant interrater variability due to the subjective nature of assigning a score to the severity of uncertainty. Respondents considered that they would spend a long time trying to assign the score. This was confirmed in the case study applications. Rating uncertainty was also deemed potentially misleading: users might mistake the resulting score for a quantitative assessment or weight. As a result, a scoring system of uncertainty was not included in TRUST.

\section{Definitions and descriptions need to be clear}

Respondents voiced difficulty with understanding the exact meaning of certain combinations of model aspects and sources of uncertainty. In response, we developed a key to the TRUST tool, with definitions and examples for each cell (Appendix 9.2).

\section{Time is the main barrier to the use of TRUST}

The main barrier to its use was the perceived time that it would take to complete TRUST. There were differing opinions on this: some interviewees thought that it was easy to fill in whilst developing or reviewing a model; others stated that this may present an additional burden for analysts and reviewers. In our case studies we found that, after some of the issues with definitions and levels were resolved, TRUST could be filled in within approximately an hour when the dossier and model were already known. Application in retrospective case studies showed that completing TRUST could be difficult and time-consuming when one had to rely on information presented in pharmacoeconomic dossiers, where uncertainty information was not easily accessible.

\subsection{The TRUST tool}

The TRUST tool integrates the outlined theoretical frameworks on model aspects and sources of uncertainty in one taxonomy (Figure 9.1). The rows present model aspects, whilst columns relate to sources of uncertainty. 


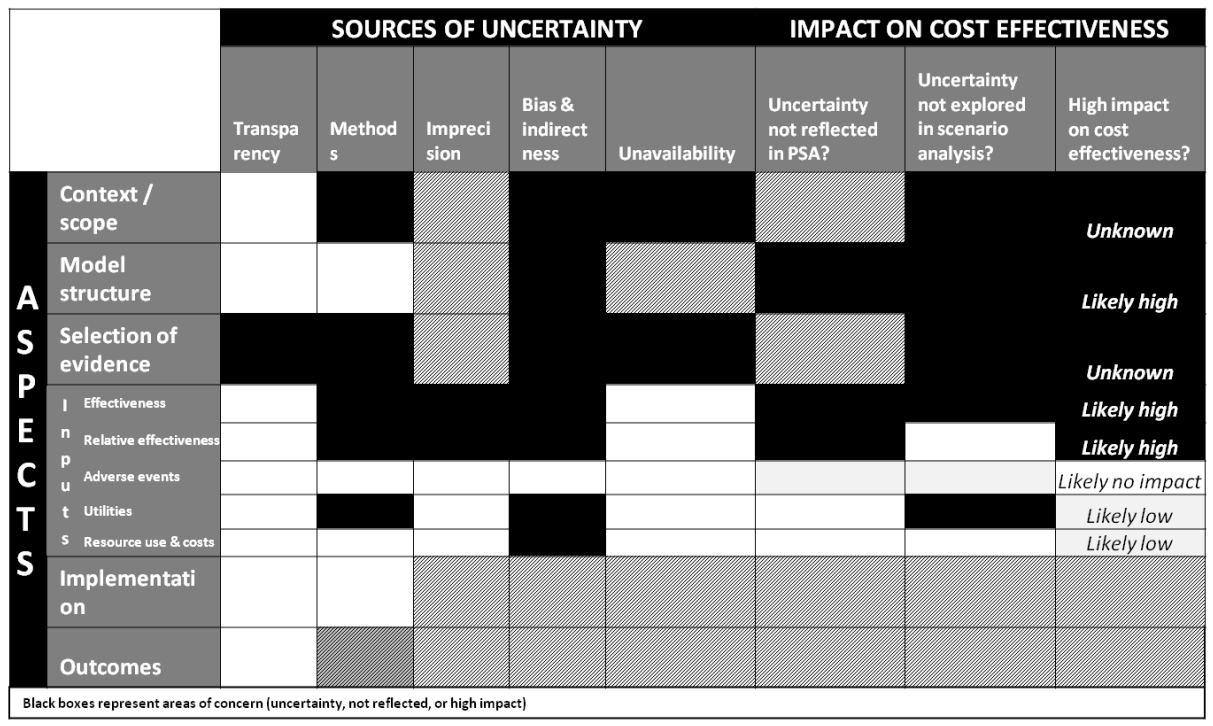

Figure 9.1: The TRUST tool part 1 - identifying uncertainty.

\subsubsection{Model aspects}

The selected model aspects include: the "context / scope", the "model structure", "selection of evidence" to inform the model, "model inputs", its "implementation" and "outcomes". Our choice of model aspects was informed by Walker et al's publication. ${ }^{27}$ Any deviations from this are in terminology used (to adapt to the target audience). With the "Scope / context" of the appraisal, we consider the definition of Patients, Intervention, Comparator, Outcomes, Time horizon, Perspective (PICOTP) of the decision problem. The aspect "Model structure" is about the selection of health states and/or events and how they relate to each other. "Selection of evidence" refers to the review(s) informing the inputs of the assessment. We have subdivided the aspect of "Model inputs" section further, distinguishing between: transition probabilities / time-to-event estimates / accuracy estimates; relative effectiveness estimates; adverse events; utilities; resource use \& costs. By "Model implementation" we refer to its technical implementation, and the calculations made. "Model outcomes" are the reported results of the model. 


\subsubsection{Sources of uncertainty}

Summarising our findings, we consider that actionable sources of uncertainty include "Transparency issues", "Methodological issues", "Imprecision", "Bias \& indirectness", and "Unavailability".

Transparency issues were included based on our experience and our interviews. We found that transparency issues were fairly common in models and their descriptions and the addition of this item was deemed valuable in interviews. Ideally, such transparency issues can be addressed early on, with clarification requests. Based on the ISPOR-SMDM taskforce report and the NUSAP framework ${ }^{28,29}$, we considered uncertainty in methods as an additional source of uncertainty to be included in TRUST: by this we refer to cases in which modelling methods are chosen that deviate from best practices or the reference case. This uncertainty could be addressed by means of clarification requests and scenario analysis.

The items of imprecision, bias \& indirectness, and unavailability are issues with the evidence itself and shed light on its quality, credibility and relevance. The item imprecision was non-controversial and was covered in all taxonomies, although with different terminology: parameter uncertainty in the ISPOR-SMDM taskforce report, direct uncertainty in van der Bles et al, statistical uncertainty (precision) in Walker et al and NUSAP, and imprecision in GRADE. ${ }^{14,18,27,29}$ In TRUST, bias \& indirectness covers multiple aspects related to issues with the evidence. The GRADE items of "study limitations", "indirectness" and "publication bias" are all included here ${ }^{14}$, as well as van der Bles' indirect uncertainty, and structural uncertainty as per the ISPOR-SMDM taskforce report. ${ }^{18,28}$ This source of evidence also includes Walker et al.'s scenario uncertainty, as potential biases may be explored through scenarios when parameterisation is not an option. ${ }^{27}$ This is best addressed using scenario analysis or elicitation of expert opinion.

We disregarded GRADE's inconsistent results, as we considered those to be issues with imprecision or bias \& indirectness, which could respectively be parameterised or explored in scenarios. ${ }^{13}$ Finally, we added unavailability to account for Walker et al's recognised ignorance, and considered that such issues could be addressed with (extreme) scenario analysis, for example informed by additional expert opinion.

\subsubsection{Combination of model aspects and sources of uncertainty}

In TRUST, combinations between aspects and sources of uncertainty are depicted in cells (Figure 9.1, and Appendix 9.2). In these cells we ask the following questions for each of the model aspects: 1 . Is there a lack of clarity in presentation, description or 
justification (transparency issue)?, 2. Is there a violation of best research practices, existing guidelines or the reference case (methodology issue)?, 3 . Is there an issue with particularly wide confidence intervals, small sample sizes or immaturity of data (imprecision)?, 4. Is there risk of bias, or indirectness (bias \& indirectness)?, 5. Is there a lack of data or insight (unavailability)? These are dichotomous questions, with "Yes" and "No" answers provided as response options. "Yes" turns the cell red, and "No" turns it green, to enable a quick graphical overview of the main uncertainties in the assessment. In addition, there is a "Not applicable" option. Furthermore, for questions 2-5, "Intransparent" can be selected when a transparency issue was reported in answering the first question. A "remarks" column allows the analyst or reviewer to provide detail on the identified uncertainties (Appendix 9.2).

Some of the combinations are more intuitive than others, and some may be downright impossible. Our experience from case studies and feedback from interviewees resulted in the following conclusions: imprecision issues are unlikely to arise within the model aspects of context / scope, model structure, and selection of evidence. Unavailability was deemed non-intuitive for the model aspect model structure. For the model aspect implementation, the relevant sources of uncertainty were transparency issues and methods issues only, and for model outcomes, we only considered transparency issues, as the others would be an aggregation of uncertainties identified in the previous model aspects. The impossible (or at least unlikely) combinations are greyed out in TRUST. For all other possible combinations, we provide explanations and examples in an explanatory file (Appendix 9.2).

\subsection{Potential impact of uncertainties on cost-effectiveness}

The next step in TRUST is the assessment of the impact on cost-effectiveness (Figure 9.2). In this section of TRUST, the analyst can indicate whether the described uncertainty is appropriately reflected in the PSA (and hence Expected Value of Perfect Information; EVPI), or not. The next question asks whether the uncertainty is explored in scenario analysis, or not.

Based on PSA and / or scenario analysis, we ask the final question: whether the identified uncertainty has an impact on cost-effectiveness. Where an uncertain aspect is parameterised and reflected in the PSA, these results can be used to identify the most impactful uncertainties. Where neither a PSA nor scenario analyses are available, a subjective judgement has to be made. The response options to this question include: "Likely high impact", "likely low impact", "likely no impact", "Unknown". Responses "Likely high impact" and "Unknown" turn these cells red. An application of the TRUST tool is shown in Box 9.2 and Table 9.1 


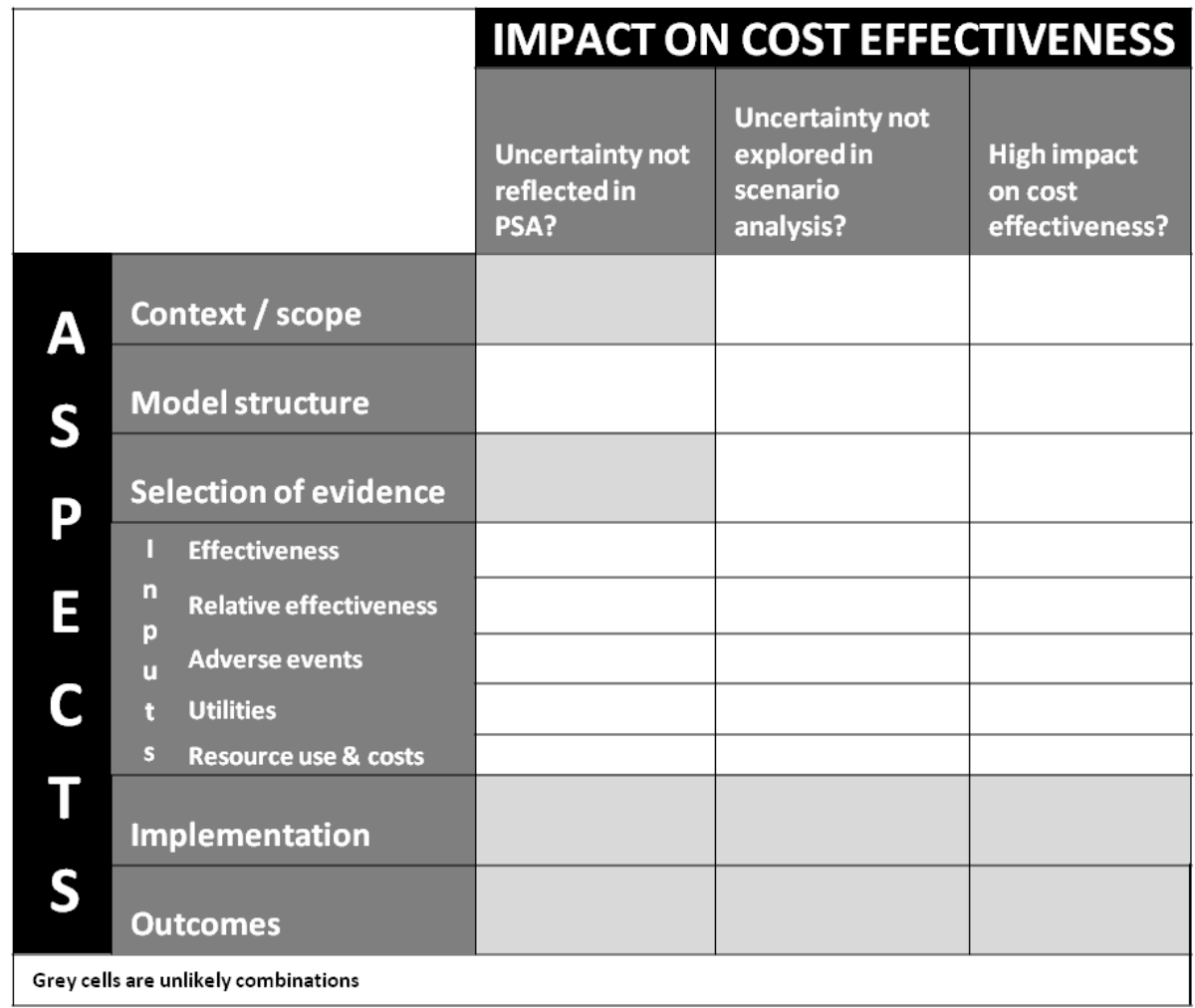

Figure 9.2: The TRUST tool part 2 - assessing impact of uncertainties on cost-effectiveness. 


\section{Box 9.2: Application of TRUST in a case study}

NICE appraisal titled "Pembrolizumab for treating relapsed or refractory classical Hodgkin lymphoma" (2018)

Pembrolizumab was compared with standard of care in patients who did and did not receive prior autologous stem cell transplant (SCT).

This NICE appraisal suffered from a lot of uncertainty (Table 9.1).

Most impactful uncertainty locations: model structure, transition probability and relative effectiveness estimates.

Model structure: the main uncertainty was that the time at which patients would receive allogeneic SCT was modelled as a fixed time point (bias) - not included in PSA, not in scenario analysis (alternative time point submitted later upon request).

Transition probability estimates: distributions to model overall survival (OS) were selected without appropriate justification (methods) - not included in the PSA but explored in scenario analysis.

The company deemed their own trial to be immature for modelling OS (imprecision) and the estimation of post-allogeneic SCT OS was based on a study, which included 13 patients (imprecision) - both included in PSA.

Relative treatment effectiveness: using a naïve comparison for two single-arm studies (methods \& bias) not in PSA, but in scenario with matched-adjusted indirect comparison.

Comparator data had a mixed population of patients that did and did not receive prior autologous SCT (bias) - neither explored in PSA nor in scenarios (scenario with alternative data for one population submitted later upon request).

Unknown impact on cost-effectiveness: context / scope and selection of evidence.

Context / scope: comparators were omitted, best supportive care (unavailability of data) and nivolumab due to direction by NICE (unavailability \& methods) - no PSA, no scenarios.

Selection of evidence: a list of health economic publications on this topic was not provided (transparency) - no PSA, no scenarios.

Conclusions:

1. Example of the increasing number of applications for reimbursement based on single-arm evidence and immature survival data.

2. Most impactful uncertainties were issues with bias \& indirectness, methods and imprecision and were not always included in the PSA.

3. Reaching a reimbursement decision under such circumstances is potentially associated with a significant risk. 


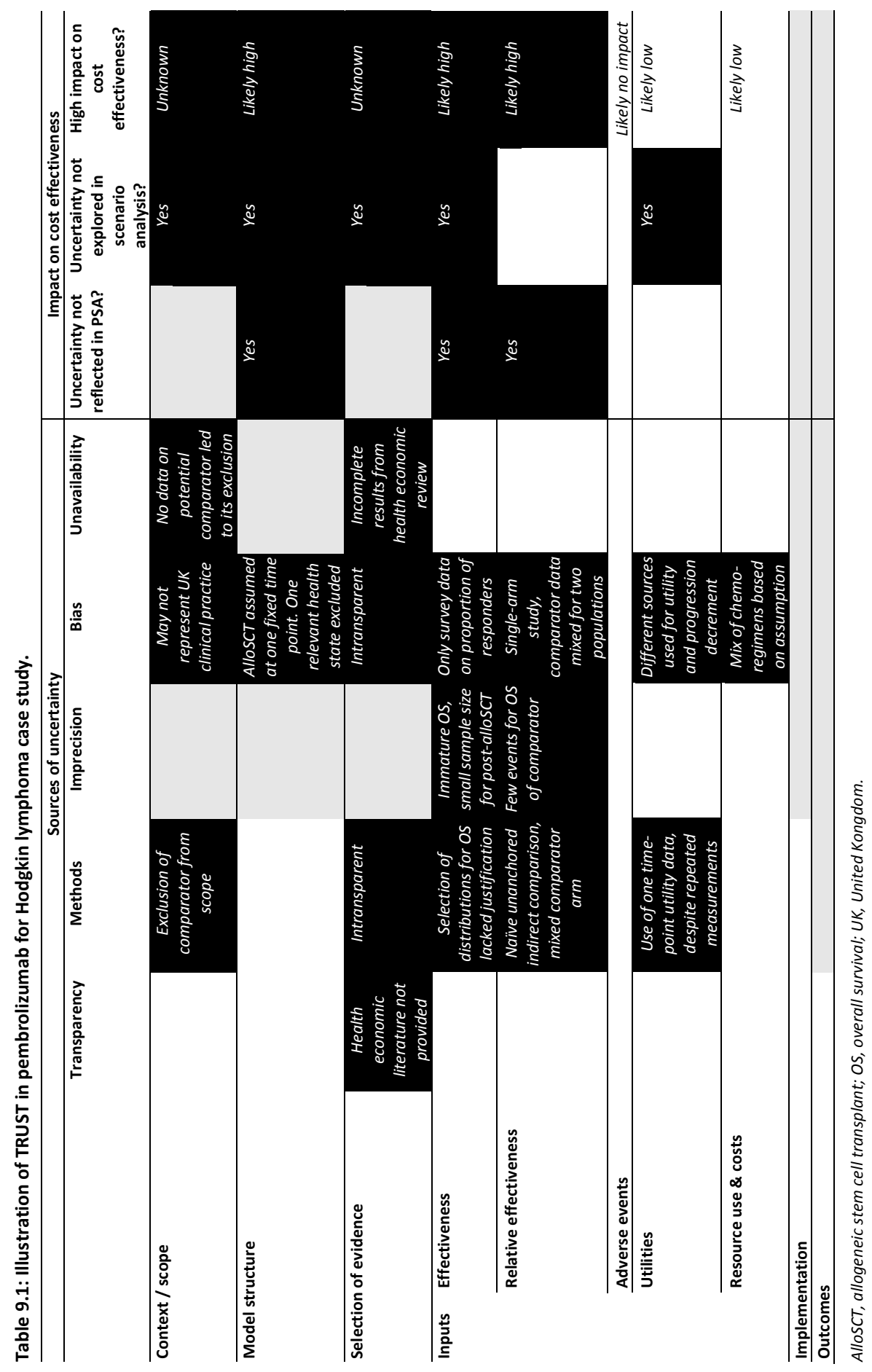




\subsection{Proposed use of TRUST in the decision-making process}

Based on the discussions with stakeholders, we think of the process of dealing with uncertainty in HTA as comprising of three phases, with specific courses of action and roles pertaining to each (Figure 9.3). The phases are: "I. Identify", "II. Explore impact", and "III. Manage".

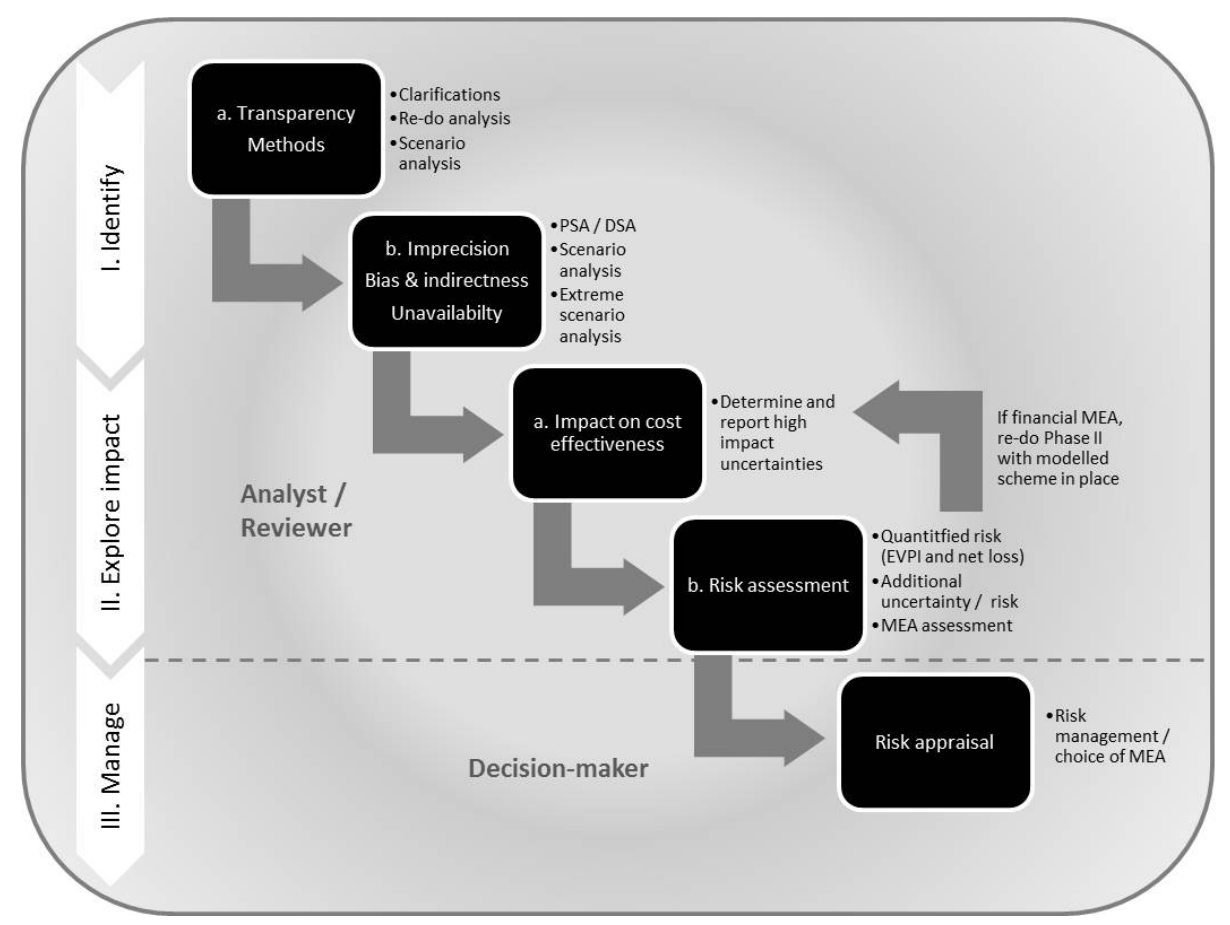

Figure 9.3: The process of uncertainty and risk assessment.

Abbreviations: $M E A=$ Managed Entry Agreement scheme, $P S A=$ probabilistic sensitivity analysis

In phase I, we first suggest identifying issues with transparency and methods (phase la). These can best be addressed by writing or requesting clarifications, redoing any analyses that were not methodologically sound, and / or performing scenario analysis on the methodological uncertainties. Next, in phase lb, uncertainties caused by imprecision, bias \& indirectness and / or unavailability are identified. These can, respectively, be addressed by probabilistic / deterministic sensitivity analysis (PSA / DSA), plausible and / or extreme scenario analysis. In addition, it is possible that issues with bias \& indirectness, unavailability and methodological issues are parameterised and therefore reflected in the PSA. 
Phase II explores the impact of uncertainty on first cost-effectiveness (Ila) and subsequently risk (IIb). An overview of high impact uncertainties can aid the design of potential research schemes, which can be assessed using established methods. ${ }^{26}$ When a financial scheme is an option, phase II is repeated with the scheme incorporated in the modelling. Phases I and II are within the responsibility of the analyst / reviewer.

Lastly, the decision-maker will appraise the risk in Phase III and determine which, if any, risk management options are most suitable and arrive at a recommendation. The TRUST tool is positioned in phases I and Ila.

\section{Discussion}

We developed the TRUST tool for systematically identifying, assessing and reporting uncertainty in health economic models. To the best of our knowledge, this is the first tool that enables the transparent identification, assessment and reporting of uncertainties in different model aspects in one place, and the first tool to highlight the different sources of uncertainties. This is important because, a) there are currently shortcomings in how uncertainty information is presented in health economic submissions, based on our experience and the literature. ${ }^{13}$ Furthermore, we hope that b) TRUST will in the future prevent uncertainties due to bias, indirectness and unavailability from being overlooked, an issue which may cause a mis-representation of risk. Based on implementation of TRUST in six case studies, we found that some of the most impactful uncertainties were not issues with imprecision but indeed with bias and indirectness. A re-thinking of how we consider uncertainties in models appears warranted, especially given that an increased leniency in evidential requirements and handling of evidence has been observed at least in the UK setting. ${ }^{30}$ Knowledge about sources of uncertainty is furthermore important because, $c$ ) it helps recognise that different sources of uncertainty may call for different types of analyses. We hope that this tool will contribute to systematically identifying, assessing and reporting uncertainties in HTA, and therefore to more informed and transparent decision-making in the face of uncertainty.

TRUST borrows from a range of uncertainty frameworks in different disciplines, which we view in two categories: those in- and those outside the field of health economics. Compared to existing uncertainty frameworks outside the field of health economics $^{16,18,27,29}$, TRUST has been adapted to match the needs of HTA analysts and decision-makers, for instance with a focus on epistemic uncertainty and it being aligned with typical structures of health economic reporting. TRUST adds to the 
frameworks inside the field of health economics ${ }^{5,28}$ by considering different sources of uncertainty and requiring an assessment for each model aspect in one place. Where other uncertainty tools have already been filled in for a certain decision problem, for instance a GRADE assessment is available for the studies informing relative treatment effectiveness, results of this can be used to fill in TRUST, if consideration is given to whether the decision problem settings are truly equivalent and the source is trustworthy. TRUST also complements existing tools on model validation. ${ }^{31,32}$ Assessing uncertainty is to be seen as a separate, although, related exercise to validating a model or model aspects. A model that is rigorously validated and even considered valid will still suffer from uncertainty. We therefore wish to caution from assuming uncertainty to be resolved when validation has taken place.

A strength of this work is that TRUST has its foundation in the uncertainty framework literature. The content of this tool was furthermore validated through feedback from experts with different backgrounds relevant to the HTA decisionmaking process during its development. Experts thought that this tool was of potential value in uncertainty assessment, but thought the proof in the pudding was in its use in actual decision-making. Application in six real-world HTA case studies served its further validation: all uncertain model aspects could be registered without difficulty and inter-rater variability was reduced after the tool's amendment. A caveat is that these six case studies may not represent all the unique features that may be observed in reimbursement applications. However, they did cover, amongst other features: single-arm studies, the use of indirect treatment comparisons, clinical evidence from different settings, structural uncertainty, long-term extrapolation beyond the available follow-up, issues related to the use of intermediate outcomes and how these related to health states.

We acknowledge that the part of TRUST concerning the impact of uncertainties on cost-effectiveness may elicit subjective answers, especially when uncertainty is not sufficiently explored through PSA or scenario analysis. However, we wish to highlight that, where these considerations are not made explicit, they will be made implicitly, and potentially in a non-systematic and opaque fashion. We consider an explicit subjective judgement to be superior to no judgement at all, or to a subconscious judgement that may implicitly drive decision-making. We recognise that representing the impact of uncertainty can be challenging, both methodologically, and regarding time constraints. We argue for parameterising uncertainty as much as possible, and, if this is not feasible, we propose representation of uncertainty in terms of ranges of the ICER or net benefit statistics. In addition, results from plausible and extreme scenario analysis and qualitative statements should be reported. ${ }^{13,18} \mathrm{~A}$ limitation of this work is the transferability of TRUST to non-UK or Dutch settings. However, the tool can be 
tweaked in the model aspects covered and response options, if interested individuals wished to apply it outside these settings and thought that model aspects did not cover all aspects considered. We believe the sources of uncertainty to be fairly universal, and grounded in the literature, so would not advise tweaking those without good justification.

A further limitation relates to the added time it may take to use TRUST. Interviewees suggested that, once in the process of reviewing evidence submitted by companies, or indeed developing an evidence submission, it might only take an hour to complete. However, we also found that this could be more difficult and therefore time-consuming if the evidence was not easily accessible. If it was not possible, in the review process, to complete TRUST, we consider this to be a potentially important finding that should be communicated, as this may mean that the evidence and the model could not be fully assessed. Furthermore, if explicit consideration of uncertainty is desired in a world that relies on increasingly sparse evidence, processes may have to be adjusted to allow for these additional analyses.

Knowledge of uncertainty is crucial for risk management. ${ }^{15,18}$ In the process of HTA, communication of uncertainty is therefore key. However, this is also a challenge. Whilst our TRUST tool can help identify and report uncertainty, effective communication to decision-makers may require effective summaries and visualisation of uncertainty and risk. It is worth noting that communicating uncertainty does not necessarily undermine trust $^{18}$, but can indeed be appreciated by the target audience. ${ }^{33}$ It can and should, in our opinion, be viewed as a duty of science ${ }^{34}$, as was expressed by Peter Høeg: "That is what we meant by science. That both question and answer are tied up with uncertainty, and that they are painful. But that there is no way around them. And that you hide nothing; instead, everything is brought out into the open." ${ }^{35}$ Further research should therefore be performed on the communication of results from TRUST to committees and decision-makers, considering whether it changes perceptions and / or behaviours. ${ }^{18,36}$ Here, both outcomes would be desirable: we wish to effect change in the perception about uncertainty and risk in an appraisal; and ideally, this would translate into a better management of risk. Further research should also focus on the link between the TRUST tool outcomes and risk management options.

\section{Conclusion}

The TRUST tool enables systematic identification, assessment and reporting of uncertainties in health economic decision models and may contribute to more 
informed and transparent decision-making in the face of uncertainty. Further research is needed on uncertainty and risk communication and the link to risk management options. 


\section{References}

1. Lipska I, Hoekman J, McAuslane N, et al. Does Conditional Approval for New Oncology Drugs in Europe Lead to Differences in Health Technology Assessment Decisions? Clin Pharmacol Ther. 2015; 98(5):489-491.

2. Grutters JPC, van Asselt MBA, Chalkidou K, et al. Healthy Decisions: Towards Uncertainty Tolerance in Healthcare Policy. Pharmacoeconomics. 2015;33(1):1-4.

3. Makady A, van Veelen A, de Boer A, et al. Implementing managed entry agreements in practice: The Dutch reality check. Health Policy. 2019;123(3):267-274.

4. Briggs $\mathrm{AH}$. Handling uncertainty in cost-effectiveness models. Pharmacoeconomics. 2000;17(5): 479-500.

5. Claxton K. Exploring uncertainty in cost-effectiveness analysis. Pharmacoeconomics. 2008;26(9): 781-798.

6. Davis C, Naci H, Gurpinar E, et al. Availability of evidence of benefits on overall survival and quality of life of cancer drugs approved by European Medicines Agency: retrospective cohort study of drug approvals 2009-13. BMJ. 2017;359(j4530).

7. Dickson R, Boland A, Duarte R, et al. EMA and NICE Appraisal Processes for Cancer Drugs: Current Status and Uncertainties. Appl Health Econ Hea. 2018;16(4):429-432.

8. Gyawali B, Hey SP, Kesselheim AS. Assessment of the Clinical Benefit of Cancer Drugs Receiving Accelerated Approval. JAMA Intern Med. 2019;179(7):906-913.

9. Sabry-Grant C, Malottki K, Diamantopoulos A. The Cancer Drugs Fund in Practice and Under the New Framework. Pharmacoeconomics. 2019;37(7):953-962.

10. Grimm SE, Armstrong N, Ramaekers BLT, et al. Nivolumab for Treating Metastatic or Unresectable Urothelial Cancer: An Evidence Review Group Perspective of a NICE Single Technology Appraisal. Pharmacoeconomics. 2019;37(5):655-667.

11. Grimm SE, Fayter D, Ramaekers BLT, et al. Pembrolizumab for Treating Relapsed or Refractory Classical Hodgkin Lymphoma: An Evidence Review Group Perspective of a NICE Single Technology Appraisal. Pharmacoeconomics. 2019;37(10):1195-1207.

12. Howard R. Information value theory. IEEE Trans Syst Sci Cybern. 1966;SSC2(1):22-6.

13. Bilcke J, Beutels $P$, Brisson $M$, et al. Accounting for Methodological, Structural, and Parameter Uncertainty in Decision-Analytic Models: A Practical Guide. Med Decis Making. 2011;31(4):675-692.

14. Guyatt $\mathrm{GH}$, Oxman $A D$, Vist GE, et al. GRADE: an emerging consensus on rating quality of evidence and strength of recommendations. BMJ. 2008;336(7650):924-926.

15. Klinke A, Renn O. A new approach to risk evaluation and management: Risk-based, precaution-based, and discourse-based strategies. Risk Anal. 2002;22(6):1071-1094.

16. van Asselt MBA, Rotmans J. Uncertainty in integrated assessment modelling: from positivism to pluralism. Climatic Change. 2002;54:75-105.

17. Balshem $H$, Helfand $M$, Schunemann $H J$, et al. GRADE guidelines: 3. Rating the quality of evidence. J Clin Epidemiol. 2011;64(4):401-406.

18. van der Bles AM, van der Linden S, Freeman ALJ, et al. Communicating uncertainty about facts, numbers and science. R Soc Open Sci. 2019;6(5):181870.

19. Briggs A, Sculpher M, Claxton K. Decision modelling for health economic evaluation. USA: Oxford University Press; 2006.

20. Iglesias $\mathrm{CP}$, Thompson A, Rogowski WH, et al. Reporting Guidelines for the Use of Expert Judgement in Model-Based Economic Evaluations. Pharmacoeconomics. 2016;34(11):1161-1172.

21. Soares MO, Sharples L, Morton A, et al. Experiences of Structured Elicitation for Model-Based CostEffectiveness Analyses. Value Health. 2018;21(6):715-723.

22. Bojke L, Claxton K, Sculpher M, et al. Characterizing Structural Uncertainty in Decision Analytic Models: A Review and Application of Methods. Value Health. 2009;12(5):739-749.

23. Strong M, Oakley JE. When Is a Model Good Enough? Deriving the Expected Value of Model Improvement via Specifying Internal Model Discrepancies. SIAM/ASA Journal on Uncertainty Quantification. 2014;2(1):106-125. 
24. Strong M, Oakley JE, Chilcott J. Managing structural uncertainty in health economic decision models: a discrepancy approach. J R Stat Soc C-Appl. 2012;61:25-45.

25. O'Hagan A, Oakley JE. Probability is perfect, but we can't elicit it perfectly. Reliab Eng Syst Safe. 2004; 85(1-3):239-248.

26. Grimm SE, Strong M, Brennan A, et al. The HTA Risk Analysis Chart: Visualising the Need for and Potential Value of Managed Entry Agreements in Health Technology Assessment. Pharmacoeconomics. 2017;35(12):1287-1296.

27. Walker WE, Harremoes $P$, Rotmans J, et al. Defining uncertainty: A conceptual basis for uncertainty management in model-based decision support. Integrated Assessment. 2003;4(1):5-17.

28. Briggs AH, Weinstein MC, Fenwick EAL, et al. Model Parameter Estimation and Uncertainty Analysis: A Report of the ISPOR-SMDM Modeling Good Research Practices Task Force Working Group-6. Med Decis Making. 2012;32(5):722-732.

29. Bouwknegt M, Havelaar A. Uncertainty assessment using the NUSAP approach: a case study on the EFoNAO tool. EFSA supporting publication. 2015;EN-663.

30. Charlton V. NICE and Fair? Health Technology Assessment Policy Under the UK's National Institute for Health and Care Excellence, 1999-2018. Health Care Anal. 2019/07/22 ed2019.

31. Eddy DM, Hollingworth W, Caro JJ, et al. Model Transparency and Validation: A Report of the ISPORSMDM Modeling Good Research Practices Task Force-7. Med Decis Making. 2012;32(5):733-743.

32. Vemer P, Ramos IC, van Voorn GAK, et al. AdViSHE: A Validation-Assessment Tool of Health-Economic Models for Decision Makers and Model Users. Pharmacoeconomics. 2016;34(4):349-361.

33. Versluis $E$, van Asselt MBA, Kim J. The multilevel regulation of complex policy problems: uncertainty and the swine flu pandemic. European Policy Analysis. 2019;5(1):80-98.

34. Saltelli A, Funtowicz S. Evidence-based policy at the end of the Cartesian dream: the case of mathematical modelling. In: Guimaraes Pereira A, Funtowicz S, editors. Science, Philosophy and Sustainability: The End of the Cartesian dream. London: Routledge; 2015.

35. Høeg P. Borderliners. Toronto: Delta Publishing; 1993.

36. Fischhoff $B$, Brewer N, Downs J. Communicating risks and benefits: an evidence-based user's guide. FDA: FDA; 2011. 


\section{Appendix 9.1}

\section{Interview guide TRUST}

\section{Introduction}

1. Presentation of the content and goal of the interview

2. Introduction of the interviewer

3. Question 1: What is your role in the health care decision making process/ the development / review of decision analytic models?

4. Short introduction of the different components of TRUST

\section{Model aspects}

[description of each aspect]

1. Question 2

a. What are your thoughts on these model aspects?

b. Are these model aspects relevant when evaluating new technologies?

c. Are there any missing aspects?

d. Are there any redundant aspects?

e. Are any of these aspects unclear or need to be reformulated?

f. Do you have any additional comments on the model aspects?

\section{Source of uncertainty (transparency and methods)}

[description of each source]

2. Question 3a

a. What are your thoughts on these sources of uncertainty?

b. Are these sources of uncertainty relevant when evaluating new technologies?

c. Are any of these sources unclear or need to be reformulated?

\section{Source of uncertainty (imprecision, bias \& indirectness, and unavailability)}

[description of each source]

3. Question 3b
a. What are your thoughts on these sources of uncertainty?
b. Are these sources of uncertainty relevant when evaluating new technologies?
c. Are there any missing sources ?
d. Are there any redundant sources?
e. Are any of these sources unclear or need to be reformulated? 
f. Do you have any additional comments on the sources of uncertainty?

\section{Scoring uncertainty}

[explanation of the scoring system]

4. Question 4

a. What are your thoughts on assigning a score to uncertainty in each model aspect?

b. Do you think it is helpful to SCORE the level of uncertainty to improve the communication of uncertainty information to stakeholders? (Yes/No and why?)

c. Do you think this is redundant to the sources of uncertainty?

d. Is this unclear or needs to be reformulated?

e. Do you foresee any barriers in SCORING the level of uncertainty?

f. Do you have any additional comments on the SCORING of uncertainty?

\section{Impact of uncertainty}

[explanation of the section relating to the impact of uncertainty]

5. Question 5

a. What are your thoughts on this section?

b. Is this section relevant when evaluating new technologies?

c. Is there any missing information?

d. Is there any redundant information?

e. Are any of these columns unclear or need to be reformulated?

f. Do these columns provide enough information to assess the impact of the identified uncertainties on decision making?

g. Do you have any additional comments on these columns?

\section{Usefulness and barriers to the use of TRUST}

6. Question 6

a. What do you think about the usefulness of this tool?

b. What do you think may be barriers to its use?

\section{Closure of the interview}

7. Question 7
a. Are there any other comments on anything related to TRUST?
b. Thank the interviewee. 



\section{Chapter 10}

General discussion 
Chapter 10 
Health technology assessments (HTAs) encompass the systematic evaluation of properties, effects and/or impacts of health care technologies. ${ }^{1}$ In multiple jurisdictions, such as the Netherlands and the United Kingdom, HTAs inform reimbursement decisions of new health care technologies, and predominantly of pharmaceuticals. Health economic models are often used in HTAs to inform decision makers about the value for money of new pharmaceuticals. The results of these health economic models are usually uncertain at the moment of reimbursement decisions because the available evidence informing these models is immature and/or incomplete. This uncertainty creates a risk of reimbursing pharmaceuticals that do not provide value for money. In this context, the aim of this thesis was to apply and develop methods to identify and assess uncertainty in HTA. More specifically, this thesis focused on the following three questions: how is uncertainty information currently considered in reimbursement decisions? How to assess uncertainty in HTA? And how to improve the uncertainty identification and assessment in HTA?

Ideally, all uncertainty surrounding the results of health economic models should be systematically identified and assessed for decision makers to appropriately manage the risk incurred by uncertainty. One methodology focussing on the systematic assessment of uncertainty surrounding health policy decisions is value of information (VOI) analysis. ${ }^{2-6} \mathrm{VOI}$ analysis quantifies the impact of uncertainty on the decision to provide an estimation of the risk associated with the decision. Indeed, the International Society for Pharmacoeconomics and Outcomes Research - Society of Medical Decision Making Taskforce on modelling good practices considers the expected value of perfect information (EVPI), the first step of a VOI analysis, as "the best measure of uncertainty surrounding a particular decision in cost-effectiveness analysis". ${ }^{7}$ Once the risk surrounding the decision is estimated, Managed Entry Agreements (MEAs) can be implemented to manage this risk. ${ }^{8-11}$ MEAs are financial or outcome-based (or a combination of both) agreements between payers and manufacturers of pharmaceuticals which may have one or more of the following objectives: manage uncertainty surrounding health policy decisions, ensure value for money of pharmaceuticals, optimise the utilisation of pharmaceuticals, or limit their budget impact. ${ }^{9}$ Coverage with evidence development (CED) is one type of MEA during which a pharmaceutical is temporarily reimbursed while additional evidence is collected on its cost effectiveness. ${ }^{12-14}$ Multiple study designs may be considered for CED research, but in the Netherlands, registries have been a common CED research design. VOI may contribute to the design of MEAs by estimating the impact of different MEAs on the risk associated with the decision. VOI is also valuable for the design of further research because it can be used to provide information on which 
element(s) of the assessment further research should focus, to evaluate the value of potential research designs, and to identify the most optimal research design. . $3,5,15^{-15}$

This chapter starts with a summary of the main findings of this thesis which provides answers to the three above-mentioned questions. Implications for research and practice concerning the use of VOI and registries to inform health policy decisions are then formulated based on these main findings. Subsequently, recommendations for practice and further research are outlined and a concluding statement finalises this chapter.

\section{Main findings}

\section{How is uncertainty information currently considered in coverage decisions?}

The first two studies of this thesis reviewed coverage decisions in two specific contexts. In Chapter 2, three Dutch coverage with evidence development (CED) cases were reviewed and stakeholders involved in these CED cases were interviewed. In all cases, CED research designs focussed on the set-up or continuation of a registry to collect daily clinical data to inform further reimbursement decision. Results showed that a systematic identification of uncertainty was lacking before CED research initiation and that there was a poor relation between the identified uncertainties and the ones included in CED research plans. VOI analysis was used in one of the three cases, but VOI results were not used for decision making. Additionally, stakeholders mentioned that CED research design should be more flexible and informed by the identified uncertainties, they further questioned whether CED research reduced the identified uncertainties.

Chapter 3 reviewed the single technology appraisal (STA) of fluocinolone acetonide intravitreal implant for the treatment non-infectious uveitis of the posterior segment. In this STA executed by the National Institute for Health and Care Excellence (NICE) in the United Kingdom, the most prominent uncertainties related to the place of fluocinolone acetonide in the treatment pathway, to the definition and measurement of the primary outcome of the trial, and to the absence of a relevant comparator, dexamethasone, in the company submission. Through multiple scenario analyses, the evidence review group showed the impact on the results of considering dexamethasone as a comparator. The impact of other uncertainties, such as the place of fluocinolone in the treatment pathway and the uncertainty surrounding the estimation of the primary outcome, could not be assessed. The appraisal committee 
acknowledged the uncertainty surrounding the results and recommended fluocinolone for the treatment non-infectious uveitis of the posterior segment in the UK.

\section{How to assess uncertainty in Health Technology Assessment?}

The following three chapters presented applications of methodologies to asses uncertainty in HTA. These applications focused on chemotherapeutic treatment for advanced breast cancer $(A B C)$. The first study was a literature review of health economic models assessing the value of chemotherapy and targeted therapy for $A B C$ (Chapter 4). In this chapter, the quality of the health economic analysis was assessed through a quality checklist and the relation between study characteristics, study quality, and study results was investigated. Most of the included studies modelled $A B C$ using a three health-state model (progression-free, progressed disease, and death). On average, the included studies satisfied 14 out of the 26 items of the quality checklist. Items of the checklist were often not fulfilled due to a lack of transparency in reporting. No relation was found between study characteristics and outcomes, except that industry-sponsored analyses seemed to result more often in favourable outcomes for the intervention than studies performed independently of industry.

Chapters $\mathbf{5}$ and $\mathbf{6}$ are applications of how registry data can be used during a HTA. The analyses presented in these chapters are based on data collected through the SOutheast Netherlands Advanced BREast cancer (SONABRE) registry (NCT03577197). Chapter $\mathbf{5}$ focused on the estimation of the relative effectiveness of eribulin (a novel chemotherapy) versus non-eribulin chemotherapy as third (or later) chemotherapy line for $A B C$, based on real-world clinical data. In total, 45 patients received eribulin and eribulin was administered as fourth or later chemotherapy line to $66 \%$ of patients. Using treatment line matching to address the potential confounding by indication introduced by the use of registry data, this study showed that eribulin did not lead to a statistically significantly increased overall survival compared with noneribulin chemotherapy. This study underlined the tolerable toxicity profile of eribulin, concluding that eribulin is an additional and tolerable treatment option for $A B C$ patients. In Chapter 6, the cost effectiveness and budget impact of eribulin versus non-eribulin as third or later treatment line for $A B C$ was investigated, using the same real-world registry data. The health economic model revealed that eribulin was not cost effective versus non-eribulin chemotherapy when considering its list price and a Dutch societal perspective. The limited number of patients who received eribulin and the lack of health-state utility values from patients who received eribulin led to uncertainty surrounding the results of this health economic evaluation. Both the budget impact and the risk (estimated through VOI analysis) associated with 
reimbursing eribulin were limited, mainly because of the small estimated number of patients who received eribulin in the Netherlands.

\section{How to improve uncertainty identification and assessment in Health Technology Assessment?}

The last three chapters of this thesis describe the development of methods and tools to improve the uncertainty assessment in HTA.

In Chapter 7, the PERSUADE template has been developed to support the selection of parametric survival models incorporated in health economic models and to render it more transparent and accessible. The PERSUADE template aims at providing the necessary input to transparently justify which parametric survival model(s) is (are) the most plausible(s) and at preventing mistakes in the implementation of parametric survival models in health economic models. This will potentially increase the transparency, internal validity, and consequently the credibility of the selected parametric survival models.

Chapter 8 focused on incorporating survival model selection uncertainty using model averaging of the probabilistic sensitivity analysis (PSA) results of the health economic model developed in Chapter 6. This study outlined a method to compute weights for model averaging which considers both statistical fit information and clinical expert opinion. The study showed that ignoring survival model selection uncertainty led to biased cost-effectiveness and uncertainty estimations. The value of reducing survival model selection uncertainty was estimated through VOI analysis. In this specific case, the value of reducing survival model selection uncertainty, estimated through the expected value of partial perfect information (EVPPI), was low because all fitted survival models led to approximately similar incremental survival estimates between intervention and comparator. However, it is expected that the EVPPI concerning survival model selection would be higher in case the fitted survival models would affect whether the intervention is deemed cost effective.

Finally, Chapter 9 describes the development and validation of the TRansparent Uncertainty ASsessmenT (TRUST) tool. TRUST enables a systematic identification, assessment and reporting of all uncertainty prevailing in all aspects (scope/context, model structure, selection of evidence, model inputs, model implementation, model outcomes) of a health economic model. TRUST highlights the source of uncertainty (transparency, methodology, imprecision, bias and indirectness, and unavailability) because different sources of uncertainty may call for different types of analyses. TRUST provides a simple overview of which uncertainties are (not) included in the PSA 
and VOI analysis. The tool may contribute to a better-informed and transparent health policy decision making under uncertainty.

\section{Implications for research and practice}

The main findings of this thesis show that there are remaining issues concerning the assessment of uncertainty in HTA which deserve more attention. These issues are fundamental to the implementation of VOI in health policy decisions, and the use of registries to inform health policy decisions.

\section{Value of Information analysis in health policy decisions}

VOI analysis is considered to provide valuable information for health policy decisions by stakeholders ${ }^{16,17}$, but it is not routinely considered during health policy decisions (Chapters $\mathbf{2}$ and $\mathbf{3}$ ). This has multiple negative consequences. Firstly, decision makers are unaware of the risk they face and may not be able to adequately manage it. ${ }^{15}$ For instance, premature reimbursement of pharmaceuticals for which the body of evidence is insufficient may lead to the reimbursement of pharmaceuticals that cause health losses. ${ }^{5}$ Secondly, only relying on expected net benefits during health policy decisions ignores the value of research that is forgone by immediate reimbursement. ${ }^{18}$ Indeed, once a positive reimbursement decision is issued, the prospect of obtaining further evidence concerning the (cost) effectiveness of a pharmaceutical through additional research is diminished. This forgone evidence may however be valuable for current and subsequent decisions. Hence, decision makers should consider the benefits of immediate reimbursement against the value of the forgone evidence. This may formally be assessed through real option analysis, a method expanding on VOI analysis which allows the comparison of the value of multiple decision options, such as immediate reimbursement, delay and perform research, or delay without further research. ${ }^{2,19}$ Thirdly, by ignoring VOI results during health policy decisions, one may engage in research that is not worthwhile. Considering VOI results during health policy decision making would thus contribute to a more efficient allocation of research budgets. For these reasons, routinely considering VOI results is warranted in health policy decision making.

For VOI analysis to provide an appropriate estimation of the risk associated with a decision and the value of further research, the impact of all uncertainty should be parameterised in the PSA. However, parameterising all uncertainty in the PSA, especially structural uncertainty, remains challenging. Chapter 8 addressed this issue 
through model averaging. Implementing model averaging may thus contribute to more complete uncertainty assessments but several barriers impede its widespread implementation. The first barrier of implementing model averaging is the elicitation of reliable estimates of the weights attributed to each scenario included in the analysis. Often, evidence concerning the plausibility of scenarios is scarce and expert opinion may be the only source of information to estimate these weights. Currently, structured expert elicitation methods are mostly used to elicit the point estimates and their surrounding uncertainty of unavailable elements of an assessment (such as survival or treatment effectiveness ${ }^{20,21}$ ) but rarely to obtain weights for model averaging. ${ }^{22,23}$ Methodological guidance concerning the elicitation of reliable weights from experts for model averaging is thus lacking. ${ }^{24}$ Since methodological choices, such as the selection of expert and the formulation of questions during the elicitation, and expert (cognitive and motivational) biases may influence the results of structured expert elicitations ${ }^{24,25}$, this area of research deserves more attention. A second barrier to using model averaging concerns which scenarios are included in model averaging. Indeed, the defined scenarios should capture all relevant uncertainty. Systematically identifying uncertainty influencing the decision before defining the different scenarios may ensure that the defined scenarios explore all relevant uncertainty. Another barrier associated with the estimation of weights for model averaging concerns the combination of expert opinion with goodness-of-fit statistics or other external data, when available. In Chapter 8, weights obtained from an expert and from goodness-offit statistics were simply averaged but one could consider to attribute more importance to weights obtained from expert elicitation or from external data. The methods to aggregate weights obtained from different sources may thus be refined. For instance, Bayesian statistics may be valuable to aggregate weights obtained from different sources based on their appropriateness for the decisions at hand. ${ }^{26}$ This appropriateness of the evidence could be systematically assessed based on the imprecision, bias, and indirectness that affect the certainty of the evidence (Chapter 9). Finally, a last barrier concerning the implementation of model averaging is the computational time that is required to perform PSAs for each included scenario, and consequently to perform VOI analysis using model averaging. This issue may however be tackled in the foreseeable future due to the increasing use of faster software such as $\mathrm{R}$ in health economic modelling ${ }^{27}$, the development of approximation methods ${ }^{28,29}$, and meta-modelling. ${ }^{30-32}$ Addressing these barriers may facilitate the implementation of model averaging and result in more complete uncertainty assessments through VOI analysis. Ultimately, this would lead to better-informed health policy decisions.

Parameterising all relevant uncertainty affecting a health policy decision in a health economic model and exploring their impact on the decision would be ideal. 
However, this will most likely remain an ideal because it is difficult or even impossible to quantify all uncertainty affecting health policy decisions. Besides, we may not be aware of all uncertainty affecting a decision ${ }^{33}$ (Chapters $\mathbf{2}$ and 9). Only considering the outcomes of health economic models and VOI analysis may thus result in ignoring important (non-quantified) uncertainties. ${ }^{34,35}$ Finally, health economic evaluations do not capture the entire value of pharmaceuticals for all stakeholders, such as obtaining access to an alternative treatment option, or bridging the time until a next breakthrough treatment for incurable disease. ${ }^{36,37}$ The main reason for this is that health economic guidelines enforce cost per quality-adjusted life years (QALY) as the primary outcome of health economic evaluations ${ }^{38-40}$, and by definition do not include all aspects of value of a pharmaceutical. Additionally, these broader aspects of value are not systematically measured and valued, and it is unclear how these aspects of value should be weighed against value for money in health policy decisions ${ }^{41,42}$. If stakeholders desire to consider these broader aspects of value in health policy decisions, a framework considering these indicators of value and their surrounding uncertainty should be developed. ${ }^{37,41}$ This would facilitate a formal appraisal of these broader aspects of value against value for money and investigate on which aspects of value further research should focus. Currently, further research mainly focuses on resource use, costs, and quality of life because these aspects are measured and assessed while broader aspects of value are currently not.

\section{Registries and health policy decision making}

In the Netherlands, CED has been used to regulate the uptake of expensive and specialised treatments between 2006 and 2012. ${ }^{43,44}$ During these CED schemes, registries have been set up or continued to collect data on the real-world clinical use, costs, and effectiveness of pharmaceuticals (Chapters 2, 5, and 6). However, Chapter $\mathbf{2}$ revealed that there was a disconnect between the identified uncertainties before CED initiation and the uncertainties included in the CED research design. This disconnect may result in uncertainties not being resolved at the end of CED research. ${ }^{45}$ The first reason for this disconnect is the lack of a systematic identification of uncertainty before CED research initiation (Chapter 2). Consequently, important uncertainties for the decision may be overlooked and CED research may not focus on the most important uncertainty for the decision. Another reason of why CED research may not focus on the most important uncertainty is the lack of structured implementation of $\mathrm{VOI}$ analysis. $\mathrm{VOI}$ analysis should be performed before CED implementation to estimate whether further research is worthwhile and to determine on which element(s) of the assessment further research should focus. This can be 
achieved by estimating the EVPI and the EVPPI for different (groups of) parameters. Afterwards, the most optimal study design can be estimated through the Expected Value of Sample Information (EVSI) and Expected Net Gain (ENG). ${ }^{46,47}$ This structured approach to decide upon the need for and design of further research is described in the literature ${ }^{4,5,12,48}$, and in the Dutch guidelines for economic evaluations in health care ${ }^{40}$, but practice shows that implementing these steps in health policy decisionmaking processes warrants more attention. A last reason explaining why CED research has not resolved all important uncertainties is that the optimal timing for reporting CED research results was not determined a priori and that regular evaluations of the value of CED research were not performed. ${ }^{49}$ Consequently, CED research may have been too short to provide valuable information, or CED research which did not focus on important uncertainty may have been continued for too long.

The disconnect between identified uncertainties and CED research has raised doubts concerning the efficiency of registries in reducing decision uncertainty. ${ }^{50}$ Registries have several potential advantages such as being cheaper to set up than randomised controlled trial, and the inclusion of patients who are more representative of patients encountered in clinical practice. ${ }^{51}$ Registries also provide valuable information about the type, effectiveness, and costs of treatments administered to patients in clinical practice ${ }^{52}$, and may thus allow a comparison between relevant alternative treatments for the decision. Registries may also capture effects and costs over a longer period of time than randomised controlled trials. ${ }^{53}$ On the other hand, using registries to inform health policy decisions is not straightforward. One main challenge concerning the use of registry data stems from the absence of randomisation of patients receiving (or not) the pharmaceutical of interest (Chapters 5 and 6), which may cause confounding by indication. ${ }^{54}$ Regressionbased methods, propensity scores ${ }^{55}$, Genetic Matching ${ }^{56}$, and instrumental variable analysis ${ }^{57}$ are existing methods to address confounding by indication. These methods aim at adjusting the comparison of the patient groups receiving the pharmaceutical and the comparator group for these disbalances in patient characteristics. Regressionbased methods, propensity scores, and Genetic Matching only correct for observed confounders, while instrumental variable analysis is also able to correct for unobserved confounders. However, finding a suitable instrumental variable is challenging and it is difficult to assess whether the instrumental variable fulfils all the necessary requirements. ${ }^{57}$ This implies that a risk of bias due to unobserved confounding remains in most non-randomised comparisons using regression-based methods, propensity scores, or Genetic Matching. To reduce this unobserved confounding when estimating the relative effectiveness of pharmaceuticals based on registry data, potential instrumental variable and confounders should be identified 
before the initiation of the registry, and be collected prospectively. Besides, the current thesis showed that the performance of current matching methods in reducing differences in baseline characteristics between the intervention and comparator groups can be improved when applied on treatment lines (Chapter 5). Since these choices are often made on an ad hoc basis, the design of a registry and the analysis of registry data may benefit from describing these choices a priori in a protocol [58]. Setting protocols for registries should include information on the objectives of the registry, and the collection and analysis of the data. Such protocols would further help to determine which health policy decisions the registry may be able to inform. Indeed, registries are not suitable to adequately inform all health policy decisions but they should be seen as complementary to randomised controlled trial evidence instead of a substitute for it. $^{52}$

This raises the question of how to use registry data in health policy decisionmaking. In the Netherlands, registries were implemented to inform whether or not to continue the reimbursement of pharmaceuticals (Chapter 6). However, discontinuation of reimbursement based on CED research results has (almost) never taken place, to the best of my knowledge. Several factors may explain why discontinuation of reimbursement is difficult to execute. Firstly, discontinuing reimbursement tend to be more difficult than not reimbursing a pharmaceutical at all [59], partly because 'losing' access to a pharmaceutical is weighted differently than obtaining access to it. ${ }^{60}$ Stakeholders seem to value the loss of access to a pharmaceutical more heavily than gaining access to treatment. ${ }^{60}$ Additionally, discontinuation of reimbursement was complicated because exit or delisting strategies were not formulated and agreed upon before the initiation of registries. ${ }^{11,61}$ Secondly, discontinuing reimbursement is complicated because policy makers evaluate the value of pharmaceuticals based on their cost per QALY while patients and clinicians may determine value of pharmaceuticals based on other criteria, such as patient satisfaction with treatment or having an alternative treatment option. There is thus a discrepancy between how value is assessed across stakeholders groups. Deciding to discontinue reimbursement based on the value for money of a pharmaceutical may create incomprehension among other stakeholder groups because the pharmaceutical may be valuable according to their value criteria. This discrepancy in how value is assessed across stakeholder groups should not be seen as a hurdle to the use of registries for informing health policy decisions, but registries should systematically gather information on these different aspects of value to ensure that these are considered during health policy decisions. Currently, the Dutch project "Regie op registers" aims at improving the use of registers to inform health policy decisions. ${ }^{62}$ This initiative is a unique opportunity to redefine how registers are used 
to inform health policy decisions and to determine which relevant outcomes for different stakeholders should be collected through registries.

\section{Recommendations for practice}

Based on the above-described implications, recommendations for practice are formulated to improve the consideration of uncertainty, and the use of VOI and registries in health policy decision making.

\section{Perform systematic uncertainty identification}

Uncertainty is not systematically considered during health policy decisions (Chapters $\mathbf{2}$ and 3) and all uncertainty cannot possibly be included in health economic models (Chapters 6, 7, and 9). To ensure all relevant uncertainty is identified and reported, uncertainty identification should be performed systematically, and early in the assessment process, to ensure it is adequately assessed later on. Uncertainty identification should ideally be based on a structured tool, such as the TRUST tool (Chapter 9). Providing a systematic overview of all uncertainty would ensure that all stakeholders consider the same uncertainties during their deliberations and may provide guidance on which uncertainty may (not) possibly be reduced through additional research.

\section{Involve all relevant stakeholders}

Implementing VOI in practice is challenging because it requires several stakeholder groups to collaborate on the assessment and appraisal of health economic evidence and the prioritization of research (Chapter 2). Currently, HTA bodies such as the Dutch Health Care Institute (Zorginstituut Nederland, ZIN) or NICE, advise about the reimbursement of new pharmaceuticals but they are not the major party tasked with the allocation of the national health research budget. Funding for health-related research is often obtained through a system of grant for which researchers have to apply on specific dates. However, health policy decision concerning CED research are not taken at specific date in a year. To ensure funding for CED research is available when policy decisions are taken, a (national) research fund should be reserved. This research fund could then be allocated outside the application deadlines for regular grants, and pharmaceutical companies should contribute to funding since they may benefit from the outcome. One example of such fund is the Cancer Drug Fund in the United Kingdom. ${ }^{63}$ A Dutch national research fund requires stakeholder groups to 
collaborate more closely than is currently the case and to define clear processes for the use of VOI and CED in practice. ${ }^{64}$ Since stakeholders may have diverging interests concerning the immediate reimbursement of a pharmaceutical or performing additional research, encouraging continuous dialogues between policy makers, patients, clinicians, researchers, pharmaceutical manufacturers, and research funding organisations through regular meetings is crucial for successfully implementing VOI in health policy decision making process. ${ }^{17,65}$

\section{Criteria for CED research}

In cases investigated in this thesis, the criteria for CED research were based on the budget impact of pharmaceuticals and whether there was uncertainty concerning their cost effectiveness in clinical practice (Chapters 2 and 6). Formal VOI analyses were not required. Supplementing current criteria for further research with VOI analyses and a structured a priori assessment of the feasibility of research may increase support among stakeholders for the CED research that will be implemented, and ensure CED research focuses on the most important uncertainty and delivers value.

\section{The use of registry data in health policy decisions}

When stakeholders decide upon collecting additional evidence through a registry, it is advised to a priori agree on multiple aspects related to the use of the registry data in health policy decision making. Firstly, a protocol concerning the aim of the registry, and the data collection and analysis plan should be written and published. ${ }^{58}$ Ideally, the aim of the registry contains an overview of uncertainty that should be reduced through additional evidence collection and how information on these uncertainties will be collected through the registry. ${ }^{66}$ When the registry is aimed at estimating the relative effectiveness of treatments, the protocol should describe which methods will be applied for handling confounding by indication. Methodological guidelines should be developed to inform decision makers and researchers designing a registry about the types of available analysis, their (dis)advantages, and how to perform them ${ }^{54,58}$, as current guidance may lack practical information on how to perform the desired analysis. $^{67}$

Secondly, stakeholders should agree on at least four points before the initiation of a registry as CED research: (a) that the evidence will be re-evaluated, (b) when the evidence will be re-evaluated, (c) how the collected evidence during CED research will be used during the re-assessment(s), (d) which health policy decision will be informed based on the additional evidence, such as continuing reimbursement, price discount 
negotiation, or optimising the use of pharmaceuticals in practice. ${ }^{61}$ During reassessments, the impact of the additional evidence on the health economic model inputs and outcomes and their surrounding uncertainty should be systematically investigated.

\section{Further research}

\section{Implementation of value of information}

VOI is not formally embedded in health policy decision making processes (Chapters 2, 3, and 6) while it may be beneficial for health policy decisions. Further research should focus on the implementation of VOI in current health policy decision-making processes. This could be achieved by performing case studies alongside prospective health policy decisions, which involve all relevant stakeholders. ${ }^{17,65}$ These case studies should first focus on identifying when VOI analysis should be used to inform health policy decisions and when health policy decisions could be taken without a (full) VOI analysis. Secondly, identifying barriers complicating the uptake of VOI analysis is essential to provide solutions to facilitate the uptake of VOI during health policy decisions. Such case studies should also focus on how VOI results can be adequately communicated and used by stakeholders and on how VOI may be implemented in current processes without dramatically increasing the time used to take a decision. By providing practical guidance on the implementation of VOI in health policy decision making processes, this type of research would contribute to increasing the use of VOI in practice.

\section{Uncertainty assessment through model averaging}

Currently, model averaging is not routinely used in health economic modelling informing health policy decisions (Chapter 7). Model averaging is however a valuable methodology to capture sources of uncertainty which are not routinely considered in PSA and hence in VOI. Its broader implementation may improve uncertainty assessment based on health economic models. Currently, methodological guidance on model averaging is limited. ${ }^{22,23}$ Further research should focus on methodological guidance concerning expert elicitation of weights attributed to scenarios included in model averaging. Eliciting weights for scenarios require specific guidance because scenarios can differ in multiple aspects from each other. For instance, scenarios may differ in structural assumptions and/or in the evidence used to inform the health 
economic model. Ensuring that the expert considers all these different aspects during the elicitation exercise is crucial to obtain reliable weights for model averaging.

Assuming we would be able to estimate weights for all plausible scenarios, two feasibility issues may hinder the use of model averaging. Firstly, the plausibility of a limited number of scenarios may be obtained through expert elicitation because elicitation exercises are cognitively burdensome and require a considerable amount of time to perform. In case experts are the only source of evidence to obtain weights, this will limit the number of scenarios that may be included in the analysis. Secondly, including all possible scenarios in model averaging may not be feasible when there is a large number of scenarios. The more scenarios are included, the longer the computational time will be. Hence, limiting the number of scenarios included in model averaging may be required. These issues pose the question on how scenarios should be selected for model averaging. Ensuring that the selected scenarios provide a complete picture of the decision problem is crucial in those cases. Before eliciting the weights of all possible scenario, one could prioritise which scenarios to focus on based on a preliminary ranking of the possible scenarios. For instance, prioritisation could be informed by a systematic identification of uncertainties and their impact on the decision. Further research on these topics should provide guidance to systematically address these issues.

\section{Communication of uncertainty}

The PERSUADE template (Chapter 8 ) supports the implementation and selection of parametric survival models and the TRUST tool (Chapter 9) enables the systematic identification, assessment, and reporting of uncertainty in health economic models. The impact of these tools on health care decisions has not yet been observed. Further research may focus on how these tools can be implemented in the best possible way and what their impact is on health policy decisions. Investigating the impact of these tools on health policy decisions may be performed by reviews of health policy decisions. The first step would be to investigate whether these tools are used in practice, and if not, identify reasons why they are not. Reviews of health policy decisions could investigate whether using PERSUADE has led to (a) more appropriate parametric survival model selection by considering all criteria included in PERSUADE, and (b) a broader acceptance of the selected survival models among stakeholders. Such review could also investigate whether using TRUST led to an improved identification, assessment, and reporting of uncertainty. For instance, one could investigate whether using TRUST has led to a more systematic consideration of uncertainty caused by the unavailability and indirectness of evidence in health policy 
decisions. The systematic investigation of the uptake and impact of these tools in health policy decisions may identify their strengths and shortcomings. These observations may ultimately lead to the improvement of these tools which may facilitate their uptake in practice and their impact on health policy decisions.

\section{Assess the value of current and future schemes}

The literature still contains a limited number of examples of formal MEA evaluations as performed in Chapter $\mathbf{2}^{8}$, hence it is unknown whether current schemes have delivered the expected benefits. Indeed, it has been argued that MEAs, especially CED, do not always reduce decision uncertainty. ${ }^{50,54}$ Further research should focus on assessing the benefits of current schemes to prevent making the same 'mistakes' all over again. ${ }^{45}$ It may also help to identify reasons why some schemes were successful, and to identify characteristics that make a specific technology suitable for CED or other MEA.

\section{Conclusion}

Uncertainty is inherent to health policy decision making regarding pharmaceuticals. The current thesis shows that uncertainty was not systematically identified and quantified, nor was the risk assessed using VOI analysis. Consequently, decision makers were not fully aware of the risk incurred by health policy decisions. Additionally, the design of registries aiming at reducing uncertainty surrounding health policy decisions did not focus on all relevant uncertainties. The current thesis therefore argues that health policy decision making could be improved by a more systematic assessment of uncertainty and risk through the structured implementation of VOI analysis. This thesis further provides a method to consider survival model selection uncertainty in $\mathrm{VOI}$ analysis through model averaging, and presents the development of the PERSUADE template and TRUST tool. The PERSUADE template aims at supporting survival model selection in health economic models, and the TRUST tool facilitates the systematic identification, assessment, and reporting of uncertainty. Embracing uncertainty through the widespread adoption of the method and tools described in the current thesis may increase stakeholders' awareness about uncertainty and the opportunities that considering uncertainty in health policy decisions may create. These opportunities concern a better management of risk, which would ultimately lead to greater health benefits being obtained with available 
resources. Finally, it would increase the value of research that is performed to inform health policy decisions. 


\section{References}

1. World Health Organisation. Medical devices > Health technology assessment [18/10/2019]. Available from: https://www.who.int/medical_devices/assessment/en/

2. Eckermann S, Willan AR. Expected value of information and decision making in HTA. Health Econ. 2007;16(2):195-209.

3. Wilson ECF. A Practical Guide to Value of Information Analysis [journal article]. PharmacoEconomics. 2015;33(2):105-121.

4. Chalkidou K, Lord J, Fischer A, et al. Evidence-based decision making: when should we wait for more information? Health Affairs. 2008;27(6):1642-1653.

5. Fenwick E, Steuten L, Knies S, et al. Value of Information Analysis for Research Decisions\&\#x2014;An Introduction: Report 1 of the ISPOR Value of Information Analysis Emerging Good Practices Task Force. Value Health. 2020;23(2):139-150.

6. Rothery $\mathrm{C}$, Strong M, Koffijberg $\mathrm{H}$, et al. Value of Information Analytical Methods: Report 2 of the ISPOR Value of Information Analysis Emerging Good Practices Task Force. Value Health. 2020;23(3):277-286.

7. Briggs $A H$, Weinstein MC, Fenwick EA, et al. Model parameter estimation and uncertainty: a report of the ISPOR-SMDM Modeling Good Research Practices Task Force--6. Value Health. 2012;15(6): 835-842.

8. Garrison LP, Jr., Towse A, Briggs A, et al. Performance-based risk-sharing arrangements-good practices for design, implementation, and evaluation: report of the ISPOR good practices for performance-based risk-sharing arrangements task force. Value Health. 2013;16(5):703-719.

9. Ferrario A, Kanavos P. Dealing with uncertainty and high prices of new medicines: a comparative analysis of the use of managed entry agreements in Belgium, England, the Netherlands and Sweden. Soc Sci Med (1982). 2015;124:39-47.

10. Carlson JJ, Sullivan SD, Garrison LP, et al. Linking payment to health outcomes: a taxonomy and examination of performance-based reimbursement schemes between healthcare payers and manufacturers. Health Policy. 2010;96(3):179-190.

11. Klemp M, Fronsdal KB, Facey K. What principles should govern the use of managed entry agreements? Int J Technol Assess Health Care. 2011 Jan;27(1):77-83. .

12. Walker S, Sculpher M, Claxton K, et al. Coverage with evidence development, only in research, risk sharing, or patient access scheme? A framework for coverage decisions. Value Health. 2012;15(3):570-579.

13. Foster NL, Mottola K, Hoffman JM. Coverage with evidence development: what to consider. JAMA Neurol. 2014;71(4):399-400.

14. Hutton J, Trueman P, Henshall C. Coverage with evidence development: an examination of conceptual and policy issues. Int J Technol Assess Health Care. 2007;23(4):425-432.

15. Grimm SE, Strong M, Brennan A, et al. The HTA Risk Analysis Chart: Visualising the Need for and Potential Value of Managed Entry Agreements in Health Technology Assessment. PharmacoEconomics. 2017;35(12):1287-1296.

16. Bindels J, Ramaekers B, Ramos IC, et al. Use of Value of Information in Healthcare Decision Making: Exploring Multiple Perspectives. PharmacoEconomics. 2016;34(3):315-322.

17. Carlson JJ, Thariani R, Roth J, et al. Value-of-information analysis within a stakeholder-driven research prioritization process in a US setting: an application in cancer genomics. Med Decis Making. 2013;33(4):463-471.

18. Griffin SC, Claxton KP, Palmer SJ, et al. Dangerous omissions: the consequences of ignoring decision uncertainty. Health Econ. 2011;20(2):212-224.

19. Grutters JPC, Abrams KR, de Ruysscher D, et al. When to wait for more evidence? Real options analysis in proton therapy. Oncologist. 2011;16(12):1752-1761.

20. Cope S, Ayers D, Zhang J, et al. Integrating expert opinion with clinical trial data to extrapolate longterm survival: a case study of CAR-T therapy for children and young adults with relapsed or refractory acute lymphoblastic leukemia. BMC Med Res Methodol. 2019 2019/09/02;19(1):182. 
21. Rossi SH, Blick C, Nathan P, et al. Expert Elicitation to Inform a Cost-Effectiveness Analysis of Screening for Renal Cancer. Value Health. 2019;22(9):981-987.

22. Jackson $\mathrm{CH}$, Bojke L, Thompson SG, et al. A framework for addressing structural uncertainty in decision models. Med Decis Making. 2011;31(4):662-674.

23. Bojke L, Claxton K, Sculpher M, et al. Characterizing Structural Uncertainty in Decision Analytic Models: A Review and Application of Methods. Value Health. 2009;12(5):739-749.

24. Bojke L, Grigore B, Jankovic D, et al. Informing Reimbursement Decisions Using Cost-Effectiveness Modelling: A Guide to the Process of Generating Elicited Priors to Capture Model Uncertainties. PharmacoEconomics. 2017;35(9):867-877.

25. Montibeller G, von Winterfeldt D. Cognitive and Motivational Biases in Decision and Risk Analysis. Risk Analysis. 2015;35(7):1230-1251.

26. Spiegelhalter DJ, Abrams KR, Myles JP. Bayesian approaches to clinical trials and health-care evaluation. Vol. 13. John Wiley \& Sons; 2004.

27. Krijkamp EM, Alarid-Escudero F, Enns EA, et al. Microsimulation Modeling for Health Decision Sciences Using R: A Tutorial. Med Decis Making. 2018;38(3):400-422.

28. Heath A, Manolopoulou I, Baio G. A Review of Methods for Analysis of the Expected Value of Information. Med Decis Making. 2017;37(7):747-758.

29. Collaborative Network for Value of Information (ConVOI). Website [19/02/2020]. Available from: https://www.convoi-group.org/resources/

30. Stevenson MD, Oakley J, Chilcott JB. Gaussian Process Modeling in Conjunction with Individual Patient Simulation Modeling: A Case Study Describing the Calculation of Cost-Effectiveness Ratios for the Treatment of Established Osteoporosis. Med Decis Making. 2004;24(1):89-100.

31. Degeling K, ljzerman MJ, Koffijberg H. A scoping review of metamodeling applications and opportunities for advanced health economic analyses. Expert Rev Pharmacoecon Outcomes Res. 2019;19(2):181-187.

32. Jalal H, Goldhaber-Fiebert JD, Kuntz KM. Computing Expected Value of Partial Sample Information from Probabilistic Sensitivity Analysis Using Linear Regression Metamodeling. Med Decis Making. 2015;35(5):584-595.

33. Walker WE, Harremoës $P$, Rotmans J, et al. Defining Uncertainty: A Conceptual Basis for Uncertainty Management in Model-Based Decision Support. Integrated Assess. 2003;4(1):5-17.

34. Grutters JP, van Asselt MB, Chalkidou K, et al. Healthy decisions: towards uncertainty tolerance in healthcare policy. PharmacoEconomics. 2015;33(1):1-4.

35. van Asselt $M$. The complex significance of uncertainty in a risk era: Logics, manners and strategies in use. Int J Risk Assess Manag. 2005 01/01;5.

36. Baltussen R, Jansen MPM, Bijlmakers L, et al. Value Assessment Frameworks for HTA Agencies: The Organization of Evidence-Informed Deliberative Processes. Value Health. 2017;20(2):256-260. d

37. Sculpher M, Claxton K, Pearson SD. Developing a Value Framework: The Need to Reflect the Opportunity Costs of Funding Decisions. Value Health. 2017;20(2):234-239.

38. National Institure for Health and Care Excellence. Guide to the methods of technology appraisal 2013. National Institure for Health and Care Excellence; 2013.

39. National Institure for Health and Care Excellence. Guide to the processes of technology appraisal (2018 update). National Institure for Health and Care Excellence; 2018.

40. Zorginstituut Nederland. Richtlijn voor het uitvoeren van economische evaluaties in de gezondheidszorg: Zorginstituut Nederland; 2015. Available from: https://www.zorginstituutnederland.nl/ over-ons/publicaties/publicatie/2016/02/29/richtlijn-voor-het-uitvoeren-van-economischeevaluaties-in-de-gezondheidszorg

41. Willke RJ, Neumann PJ, Garrison LP, Jr., et al. Review of Recent US Value Frameworks\&\#x2014;A Health Economics Approach: An ISPOR Special Task Force Report [6]. Value Health. 2018;21(2): 155-160.

42. Neumann PJ, Willke RJ, Garrison LP, Jr. A Health Economics Approach to US Value Assessment Frameworks\&\#x2014; Introduction: An ISPOR Special Task Force Report [1]. Value Health. 2018;21(2): 119-123. 
43. Zorgautoriteit N. BELEIDSREGEL BR 2011 [01/04/2020]. Available from: https://puc.overheid.nl/ nza/doc/PUC_20840_22/

44. Nederland Z. mw. dr. G. Ligtenberg. Voorwaardelijke toelating/financiering van zorg 2012 [01/04/2020]. Available from: https://www.zorginstituutnederland.nl/publicaties/rapport/ 2012/04/06/voorwaardelijke-toelating-financiering-van-zorg

45. Makady A, van Veelen A, de Boer A, et al. Implementing managed entry agreements in practice: The Dutch reality check. Health Policy. 2019;123(3):267-274.

46. Eckermann S, Karnon J, Willan AR. The Value of Value of Information [journal article]. PharmacoEconomics. 2010;28(9):699-709.

47. Heath A, Kunst N, Jackson C, et al. Calculating the Expected Value of Sample Information in Practice: Considerations from 3 Case Studies. Med Decis Making. 2020:40(3):314=326.

48. Claxton K, Palmer S, Longworth L, et al. A Comprehensive Algorithm for Approval of Health Technologies With, Without, or Only in Research: The Key Principles for Informing Coverage Decisions. Value Health. 2016;19(6):885-891.

49. Mohseninejad L, van Gils C, Uyl-de Groot CA, et al. Evaluation of Patient Registries Supporting Reimbursement Decisions: The Case of Oxaliplatin for Treatment of Stage III Colon Cancer. Value Health. 2015;18(1):84-90.

50. Franken MG, Gaultney JG, Blommestein HM, et al. Policymaker, please consider your needs carefully: does outcomes research in relapsed or refractory multiple myeloma reduce policymaker uncertainty regarding value for money of bortezomib? Value Health. 2014;17(2):245-253.

51. Garrison LP, Jr., Neumann PJ, Erickson P, et al. Using real-world data for coverage and payment decisions: the ISPOR Real-World Data Task Force report. Value Health. 2007;10(5):326-335.

52. Fleurence RL NH, Jansen JP. The Critical Role Of Observational Evidence In Comparative Effectiveness Research. Health Affairs. 2010;29(10):1826-1833.

53. Sculpher MJ, Claxton K, Drummond M, et al. Whither trial-based economic evaluation for health care decision making? Health Econ. 2006;15(7):677-87.

54. Blommestein HM, Franken MG, Uyl-de Groot CA. A Practical Guide for Using Registry Data to Inform Decisions About the Cost Effectiveness of New Cancer Drugs: Lessons Learned from the PHAROS Registry. PharmacoEconomics. 2015;33(6):551-560.

55. Rosenbaum PR, Rubin DB. The Central Role of the Propensity Score in Observational Studies for Causal Effects. Biometrika. 1983;70(1):41-55.

56. Sekhon JS, Grieve RD. A matching method for improving covariate balance in cost-effectiveness analyses. Health Econ. 2012;21(6):695-714.

57. Angrist JD, Imbens GW, Rubin DB. Identification of Causal Effects Using Instrumental Variables. J Am Stat Assoc. 1996;91(434):444-455.

58. Berger ML, Sox H, Willke RJ, et al. Good practices for real-world data studies of treatment and/or comparative effectiveness: Recommendations from the joint ISPOR-ISPE Special Task Force on realworld evidence in health care decision making. Pharmacoepidemiol Drug Saf. 2017;26(9):1033-1039.

59. van de Wetering EJ, van Exel J, Brouwer WBF. The Challenge of Conditional Reimbursement: Stopping Reimbursement Can Be More Difficult Than Not Starting in the First Place! Value Health. 2017;20(1):118-125.

60. Rotteveel AH, Lambooij MS, Zuithoff NPA, et al. Valuing Healthcare Goods and Services: A Systematic Review and Meta-Analysis on the WTA-WTP Disparity. PharmacoEconomics. 2020;38(5):443-458.

61. Hoekman J, Boon W, Bouvy J, et al. Use of the conditional marketing authorization pathway for oncology medicines in Europe. Clin Pharmacol Ther. 2015;98(5):534-541.

62. Zorginstituut Nederland. Regie op Registers voor dure geneesmiddelen 2019 [06/02/2020]. Available from: https://www.zorginstituutnederland.nl/werkagenda/regie-op-registers-voor-dure-geneesmiddelen

63. Mayor S. New "managed access" process for Cancer Drugs Fund to go ahead, NHS England confirms. BMJ. 2016;352:i1208.

64. Brugger U. A review of Coverage with Evidence Development (CED) in different countries: What works and what doesn't 2014 [14/04/2020]. Available from: https://htai.org/wpcontent/uploads/2018/02/CED_Report_Bruegger_Final_Version.pdf 
65. Bennette CS, Veenstra DL, Basu A, et al. Development and Evaluation of an Approach to Using Value of Information Analyses for Real-Time Prioritization Decisions Within SWOG, a Large Cancer Clinical Trials Cooperative Group. Med Decis Making. 2016;36(5):641-651.

66. Morrell L, Wordsworth S, Schuh A, et al. Will the reformed Cancer Drugs Fund address the most common types of uncertainty? An analysis of NICE cancer drug appraisals. BMC Health Serv Res. 2018;18(1):393.

67. Faria R, Hernandez Alava M, Manca A, et al. NICE DSU Technical Support Document 17: The use of observational data to inform estimates of treatment effectiveness for Technology Appraisal: Methods for comparative individual patient data 2015. Available from: http://www.nicedsu.org.uk 

Summary 
Addenda 
Reimbursement decisions concerning health care technologies are made under uncertainty because decision makers wish to rapidly make health care technologies available to patients while the evidence supporting these decisions is often immature and/or incomplete. As a consequence of this uncertainty, pharmaceuticals that do not provide value for money may be reimbursed, while pharmaceuticals providing value for money may not be reimbursed through health insurance packages. The aim of this thesis was to apply and develop methods to identify and assess uncertainty in health technology assessments (HTA). HTA aims at informing decision makers about the value of health care technologies through a systematic assessment of properties, effects, and/or impacts of these health care technologies.

Model-based health economic evaluations play a prominent role in reimbursement decisions because they facilitate data synthesis, data extrapolation, and allow to include all relevant comparators for the decision of interest. Health economic models further allow to assess the uncertainty surrounding the cost effectiveness of pharmaceuticals. One method used to assess the uncertainty surrounding health policy decisions is value of information (VOI) analysis. VOI analysis quantifies the impact of uncertainty (combining the probability and consequences of reimbursing a cost-ineffective health care technology) to provide an estimation of the risk associated with the decision at hand. Once the risk surrounding the decision is estimated, financial and/or outcome-based Managed Entry Agreements (MEAs) can be implemented to manage it. MEAs are agreements between payers and pharmaceutical companies aiming at providing access to a pharmaceutical while managing the uncertainty surrounding their (cost) effectiveness, and/or budget impact. Coverage with evidence development (CED) is a type of MEA during which a pharmaceutical is temporarily reimbursed and additional evidence is collected concerning its (cost) effectiveness. This additional evidence aims at taking a betterinformed after the CED period. In the Netherlands, registries are a common CED research design. VOI is valuable for the design and prioritisation of further research because it can be used to provide information on which element(s) of the assessment further research should focus, to evaluate the value of potential research designs, and to identify the most optimal research design. VOI can contribute to the design of MEAs by estimating the impact of different MEAs on the risk of reimbursing pharmaceuticals.

The first part of this thesis describes how uncertainty is currently considered in reimbursement decisions. Chapter 2 investigates which uncertainties were present before the initiation of CED research and whether these uncertainties were included in CED research plans. Stakeholders involved in these CED schemes were interviewed on issues and ways forward with uncertainty in CED schemes. In all three investigated 
CED schemes, CED research designs focused on the set-up or continuation of registries to collect clinical data to inform reimbursement continuation. A systematic identification of uncertainty was lacking before CED research initiation and there was a poor relation between the uncertainties present before CED initiation and those included in the CED research plans. VOI analysis was performed in one case, but VOI results did not inform decision making. Stakeholders argued for more flexible CED research design which should be informed by uncertainties present before CED initiation. Stakeholders questioned whether CED research reduced the identified uncertainties. Chapter 3 presents a review of the single technology appraisal concerning fluocinolone acetonide intravitreal implant for treating non-infectious uveitis of the posterior segment in the United Kingdom. This chapter describes which uncertainty influenced the cost-effectiveness results and the reimbursement decision during this appraisal. Prominent uncertainties concerned the place of fluocinolone acetonide in the treatment pathway, the definition and measurement of the primary outcome of the pivotal trial, and the absence of a relevant comparator (dexamethasone) in the company submission. Through scenario analyses, the evidence review group showed the impact on the results of considering dexamethasone as a comparator. The impact of other uncertainties, such as the place of fluocinolone in the treatment pathway, could not be assessed. The appraisal committee acknowledged the uncertainty surrounding the results. Considering the range of results, the appraisal committee concluded that fluocinolone was a costeffective use of National Health Service resources. Fluocinolone acetonide intravitreal implant was recommended for treating non-infectious uveitis of the posterior segment.

The second part of this thesis focuses on applying different methods to assess uncertainty. All methods are applied to case studies considering the assessment of chemotherapy for the treatment of advanced breast cancer $(A B C)$ patients. Chapter 4 is a literature review of model-based health economic evaluations concerning $A B C$ treatments. This chapter investigates the relation between study characteristics and study quality and outcomes. On average, the included studies satisfied 14 out of the 26 items of a well-known quality checklist. Items of the checklist were often not fulfilled due to a lack of transparency in reporting. No relation was found between study characteristics and outcomes, except that industry-sponsored analyses seemed to result more often in favourable outcomes for the intervention than studies performed independently of industry. In Chapter 5, the relative effectiveness of eribulin (a novel chemotherapy) versus non-eribulin chemotherapy for the treatment of $A B C$ patients is estimated using data collected through the SOutheast Netherlands Advanced BREast cancer (SONABRE) registry (NCT03577197). Forty-five patients 
received eribulin. Eribulin was administered as a late treatment line to most patients. Since registry data informed this study, uncertainty arose due to the absence of randomisation and the potential bias induced by confounding by indication. Genetic Matching was applied on treatment lines administered to patients to address this bias. Using treatment line matching, eribulin did not lead to a statistically significantly increased overall survival compared with non-eribulin chemotherapy (hazard ratio: $0.66,95 \%$ confidence interval: $0.38-1.13)$. This study emphasised the tolerable toxicity profile of eribulin. Chapter 6 builds upon Chapter 5 and estimates the cost effectiveness of eribulin versus non-eribulin chemotherapy, using a health economic model. The budget impact and risk (estimated through VOI analysis) associated with reimbursing eribulin were also estimated. The estimated incremental costeffectiveness ratio (ICER) was $€ 220,608$ per quality-adjusted life-year gained versus non-eribulin chemotherapy. This ICER exceeds the Dutch willingness-to-pay thresholds. Uncertainty surrounding the results arose due to the limited number of patients receiving eribulin and the lack of health-state utility values estimated in patients receiving eribulin. Both budget impact and risk of reimbursing eribulin are limited, mainly because of the small estimated number of patients who may receive eribulin in the Netherlands.

The third part of this thesis focuses on developing methods and tools to improve the identification and assessment of uncertainty in HTAs. In Chapter 7, the development of the ParamEtRic SUrvivAl moDel sElection (PERSUADE) template is described. This template aims at supporting the selection of parametric survival models incorporated in health economic models and at rendering it more transparent and accessible. The PERSUADE template provides the necessary input to transparently justify which parametric survival model(s) is (are) the most plausible and may help to prevent mistakes in the implementation of parametric survival models in health economic models. This will potentially increase the consistency, transparency, internal validity, and consequently the credibility of the selected parametric survival models. Chapter 8 describes a method to capture structural uncertainty stemming from survival model selection in health economic models by applying model averaging of the probabilistic sensitivity analysis (PSA) results of the health economic model developed in Chapter 6. This study outlines a method to compute weights for model averaging which considers both statistical fit information and clinical expert opinion. Ignoring survival model selection uncertainty led to biased cost-effectiveness results and uncertainty estimations. The value of reducing survival model selection uncertainty was estimated through VOI analysis. In this specific case, the value of reducing survival model selection uncertainty was low because all fitted parametric survival models led to approximately similar incremental survival estimates. This 
result is likely case-specific and could be substantially different in other assessments. Chapter 9 describes the development and validation of the TRansparent Uncertainty ASsessmenT (TRUST) tool. TRUST enables a systematic identification, assessment, and reporting of all uncertainty prevailing in all aspects (scope/context, model structure, selection of evidence, model inputs, model implementation, model outcomes) of a health economic model. TRUST highlights the source of uncertainty (transparency, methodology, imprecision, bias and indirectness, and unavailability) since different sources of uncertainty may call for different types of analyses and health policy decisions to manage these uncertainties. TRUST also provides a simple overview of uncertainties which are (not) included in the PSA and VOI analysis.

In the Netherlands, registries were implemented as part of CED schemes to regulate the uptake of expensive and specialised treatments between 2006 and 2012. These registries aimed at reducing the uncertainty surrounding the cost effectiveness of these treatments through data collection concerning their real-world clinical use, costs, and effectiveness. VOI, which can contribute to the design of MEAs, was not routinely used to inform the design of these registries. The general discussion of this thesis therefore focuses on two subjects: a) the importance of VOI analysis for health policy decisions, and b) the use of registries to inform health policy decisions. Regarding the importance of VOI analysis for health policy decisions, this thesis argued that not considering VOI analysis in health policy decisions has multiple negative consequences. By not considering VOI analysis results, decision makers are unaware of the risk they face and may thus not be able to adequately manage it. Also, research which is not worthwhile may be commissioned because the value of additional research is not investigated. Routinely considering VOI results is therefore warranted to inform health policy decision making. Implementing VOI in practice is however challenging because performing VOI analysis may be time consuming and it requires multiple stakeholders to interact with each other, ideally in an iterative way. More research is needed to determine how to implement VOI analysis in practice. Concerning the technical implementation of VOI, further research should focus on improving guidance on the implementation of model averaging, to ensure future VOI analysis provide more complete assessment of uncertainty than currently performed. Nevertheless, the ideal situation where all uncertainty surrounding a health policy decision is incorporated in VOI analyses may never be achieved for two reasons: a) we may not be aware of all uncertainty affecting a decision, b) health economic evaluations do not capture all aspects of value of pharmaceuticals because they operationalise value in cost per quality-adjusted life years gained. It is currently still unclear how these broader aspects of value of pharmaceuticals should be weighed against value for money in health policy decisions. If stakeholders desire to consider 
these broader aspects of value in health policy decisions, a framework considering these indicators of value and their surrounding uncertainty should be developed.

Secondly, the use of registries to inform health policy decisions is discussed. We identified a disconnect between the uncertainties present before CED initiation and the ones included in CED research plans. A reason explaining this disconnect is the lack of systematic uncertainty identification before CED initiation. Consequently, CED research may not focus on the most important uncertainty for the decision. A systematic identification of uncertainty, through the use of TRUST for instance, is warranted to prevent that important uncertainty are overlooked and future registries focus on the most important uncertainty. Registries have several advantages above performing randomised controlled trials such as being cheaper and including patients who are representative of patients encountered in clinical practice. However, the analysis of registry data is challenging due to the absence of randomisation, potentially causing confounding by indication. Statistical methods have been developed to address this bias but analysis choices are often made ad hoc when the registry data has already been collected. Describing analysis choices a priori in a protocol may improve the design of registries and the analysis of registry data. Methodological guidelines should be developed to inform decision makers and researchers designing a registry about the available types of analysis, their (dis)advantages, and how to perform them.

In conclusion, the current thesis has argued that health policy decision making could be improved by a more systematic assessment of uncertainty and risk through a structured implementation of VOI analysis and the use of registry data. This thesis shows how registry data can be used to assess the (cost) effectiveness of a pharmaceutical, and provides a method and tools to improve uncertainty identification, assessment, and reporting during the assessment of health care technologies. Embracing uncertainty through the widespread adoption of the method and tools described in the current thesis may increase stakeholders' awareness about uncertainty and the opportunities that considering uncertainty in health policy decisions may create. These opportunities encompass a better management of risk, which would ultimately lead to greater health benefits being obtained with the available resources. Finally, it would increase the value of research that is performed to inform health policy decisions. 
Addenda 
Valorisation 
Addenda 
Health Technology Assessment (HTA) is a field aiming at informing decision makers about the value of health care technologies and has direct policy relevance as well as societal impact. ${ }^{1-3}$ This emphasises that the analyses, tools, and method developed in the current thesis are not merely scientific exercises. The overall aim was to provide tools to improve the consideration of uncertainty during the assessments of health care technologies, likely leading to better-informed health policy decisions in the future. In this chapter, I discuss how the results of the current thesis have generated value and may continue to generate value in- and outside the scientific community. Value is defined in this context as "the process of creating value from knowledge, by making knowledge suitable and/or available for social (and/or economic) use and by making it suitable for translation into competing products, services, processes and new activities". 4

I will first describe the societal relevance of the current thesis for different stakeholders, and describe why and how the different products developed in the current thesis are innovative. This chapter is concluded with the steps that have been undertaken to disseminate the results of the current thesis.

\section{Societal relevance}

This section discusses the relevance of the results of this thesis for different stakeholder groups, namely health care professionals and patients, health policy decision makers, and HTA and health economics researchers.

First of all, the results of the current thesis have had a direct impact on care that can be provided by health care professionals to patients. The study describing the appraisal of fluocinolone acetonide implant (Chapter $\mathbf{3}$ ) is a summary of an actual appraisal which has resulted in the recommendation of fluocinolone acetonide intravitreal implant for the treatment of recurrent non-infectious uveitis in the United Kingdom. All documents that were produced during this appraisal are available online ${ }^{5}$, however, these documents are lengthy. By writing this publicly available summary, we hope to have provided a clear overview of the key uncertainties that arose during this appraisal and of the reasons why fluocinolone acetonide intravitreal implant was finally recommended. The subsequent chapters of this thesis also aim at impacting patients care by improving the consideration of uncertainty in health policy decisions. Systematically considering and assessing a broader range of uncertainty would result in better-informed health policy decisions, and facilitate (rapid) access to health care technologies providing value for money, and hopefully value to patients. 
The second stakeholder group which benefits from the results of the current thesis are health policy decision makers. They directly benefited from our expertise, as an evidence review group, during the appraisal of fluocinolone acetonide intravitreal implant (Chapter 3). Additionally, Chapter 2 argues that the implementation of registries and coverage with evidence development (CED) may benefit from a systematic identification of uncertainty before their implementation. This may improve the design of CED schemes by focusing on remaining uncertainties. The general discussion of this thesis further encourages the use of value of information (VOI) to inform CED research design and provides recommendations about how to improve the implementation of $\mathrm{VOI}$ and registries to inform health policy decisions. For instance, the set-up of mandatory protocols could contribute to improving the design of registries. The tools and method described in Chapters $\mathbf{7}$ to 9 aim at improving the uncertainty identification and assessment in health economic evaluations and health policy decisions. The broader uptake of these tools and method in company submissions, or during the review of such submission may lead to more complete assessment of uncertainty than in current submissions.

Finally, HTA and health economics researchers benefit from the results of the current thesis. Chapters $\mathbf{5}$ and $\mathbf{6}$ provide examples of how registry data can be used and analysed to assess the effectiveness and cost effectiveness of pharmaceuticals. Additionally, the tools and method developed in Chapters $\mathbf{7}$ to $\mathbf{9}$ will be made freely available online for use once published. Using these tools systematically during model development may lead to more complete uncertainty assessment. Additionally, these tools encourage a more systematic reporting of which uncertainty is present and assessed during the assessment of health care technologies.

\section{Products and innovativeness}

Chapters 6 to 9 resulted in innovative products described in this section. These product are partly already freely available online and the remainder will be made available online once published.

The health economic model developed in Chapter 6 is freely available online. ${ }^{6}$ This is innovative since health economic models are not routinely published online. We therefore contributed to open sciences by providing access to the source code, and consequently allow the re-use of (part of) the code for further assessments.

The ParamEtRic SUrvivAl moDel sElection (PERSUADE) template (Chapter 7) is also freely available online (https://github.com/Bram-R/PERSUADE), and is the first 
template aiming at standardising parametric survival model selection for health economic models.

The $\mathrm{R}$ code to estimate weights for multiple parametric survival models is described in Chapter 8. Survival model selection uncertainty is currently not routinely included when assessing the uncertainty surrounding health economic models. By providing this code, we hope to encourage health economic modellers to consider this uncertainty during their assessment.

Finally, Chapter 9 describes the development of the TRansparent Uncertainty ASsessmenT (TRUST) tool, which is also freely available to anyone as an Appendix to the open source publication (https://doi.org/10.1007/s40273-019-00855-9). ${ }^{7}$ To the best of my knowledge, no such tools promoting a transparent and systematic identification of uncertainty existed within the HTA literature.

\section{Dissemination}

The results of this thesis have been disseminated among the different stakeholder groups through different activities.

Almost all papers described in the current studies have been published open access in international scientific journals. This has permitted their dissemination among researchers but provides access to their content to all stakeholders if they desire to. Additionally, a short and non-technical article describing the advantage of treatment-line matching (Chapters 5 and 6) has been published in a non-peer reviewed journal (Value and Outcome Spotlight) published by the International Society for Pharmacoeconomics and Outcomes Research (ISPOR). The complete report summarised in Chapter $\mathbf{3}$ can also be freely accessed on the National Institute for Health and Clinical Excellence website. ${ }^{5}$ Besides (open access) scientific publications, the products developed in Chapters 6 to 9 will become available online once published.

Research performed as part of this thesis has also been presented at (inter)national conferences, which ensured their dissemination among the scientific community, employees of pharmaceutical and consultancy firms, as well as decision makers. The results of the current studies have been presented during ISPOR Europe, the European Health Economic Association (EUHEA) congress, the International Health Economic Association (iHEA congress), the Lowlands Health Economics Study Group, the Care and Public Health Research Institute (CAPHRI) Research Day, the 'Certainly uncertain' symposium of the Nederlands Vereniging voor Technologie Assessment in 
de Gezondheidszorg (NVTAG), and the national symposium of the Vereniging voor Gezondheidseconomie (VGE).

To ensure the dissemination of TRUST among pharmacoeconomic assessors of the Dutch Health Care Institute (Zorginstituut Nederland) and thus during Dutch health policy decisions, TRUST has been applied during a prospective case study. ${ }^{8}$ Additionally, TRUST has been used and presented during the appraisal of tomosynthesis as a screening instrument for the Dutch National Breast Cancer Screening Program. This ensured the dissemination of TRUST among decision makers. A workshop on uncertainty has also been held to present TRUST to a wide audience of stakeholders involved in the assessment and appraisal of health care technologies in the Netherlands and the United Kingdom (pharmacoeconomic assessors, decision makers, HTA researchers). 


\section{References}

1. World Health Organisation. Medical devices > Health technology assessment [18/10/2019]. Available from: https://www.who.int/medical_devices/assessment/en/

2. Liberati A, Sheldon TA, Banta HD. EUR-ASSESS Project Subgroup report on Methodology. Methodological guidance for the conduct of health technology assessment. Int J Technol Assess Health Care. 1997;13(2):186-219.

3. O'Rourke B, Oortwijn W, Schuller T. The new definition of health technology assessment: A milestone in international collaboration. Int J Technol Assess Health Care. 2020;36(3):187-790.

4. Landelijke Commissie Valorisatie. Waardevol: Indicatoren voor Valorisatie. Den Haag: Rathenau Instituut; 2011.

5. National Institure for Health and Care Excellence. Fluocinolone acetonide intravitreal implant for treating recurrent non-infectious uveitis>History 2019 [11/07/2020]. Available from: https://www.nice.org.uk/guidance/ta590/history

6. Pouwels XGLV, Ramaekers BLT, Geurts SME, et al. An economic evaluation of eribulin for advanced breast cancer treatment based on the Southeast Netherlands advanced breast cancer registry. Acta Oncologica. 2020:1-8.

7. Grimm SE, Pouwels X, Ramaekers BLT, et al. Development and Validation of the TRansparent Uncertainty ASsessmenT (TRUST) Tool for Assessing Uncertainties in Health Economic Decision Models. PharmacoEconomics. 2020;38(2):205-216.

8. Zorginstituut Nederland. Pakketadvies sluisgeneesmiddel voretigene neparvovec (Luxturna ${ }^{\circledR}$ ) bij de behandeling van visusverlies door erfelijke retinale dystrofie met bi-allelische RPE65-mutaties. 2020. 
Addenda 


\section{List of publications and presentations}


Addenda 


\section{Scientific publications}

Pouwels XGLV, Geurts SME, Ramaekers BLT, Erdkamp F, Vriens BEPJ, Aaldering KNA, van de Wouw AJ, Dercksen MW, Smilde TJ, Peters NAJB, van Riel JM, Pepels MJ, Heijnen-Mommers J, Tjan-Heijnen VCG, de Boer M, Joore MA (2020) An economic evaluation of eribulin for advanced breast cancer treatment based on the Southeast Netherlands advanced breast cancer registry. Acta Oncol .Jun 16;1-8. doi: 10.1080/0284186X.2020.1775289. [Online ahead of print]

Schneider PP, Pouwels XGLV, Passos VL, Ramaekers BLT, Geurts SME, Ibragimova KIE, de Boer M, Erdkamp F, Vriens BEPJ, van de Wouw AJ, den Boer MO, Pepels MJ, TjanHeijnen VCG, Joore MA. Variability of Cost Trajectories Over the Last Year of Life in Patients With Advanced Breast Cancer in the Netherlands. PLoS One. 2020. Apr 9;15(4):e0230909. doi: 10.1371/journal.pone.0230909.

Pouwels XGLV, Petersohn S, Carrera VH, Denniston AK, Chalker A, Raatz H, Armstrong N, Witlox W, Worthy G, Noake C, Riemsma R, Kleijnen J, Joore MA. Response to Comment on "Fluocinolone Acetonide Intravitreal Implant for Treating Recurrent NonInfectious Uveitis: An Evidence Review Group Perspective of a NICE Single Technology Appraisal". Pharmacoeconomics. 2020 Jun;38(6):649-651. doi: 10.1007/s40273-02000900-y.

Petersohn S, Pouwels X, Ramaekers B, Ten Cate-Hoek A, Joore M. Rivaroxaban Plus Aspirin for the Prevention of Ischaemic Events in Patients With Cardiovascular Disease: A Cost-Effectiveness Study. Eur J Prev Cardiol. 2020 Mar 29;2047487320913380. doi: 10.1177/2047487320913380.

Grimm SE, Pouwels X, Ramaekers BLT, Wijnen B, Knies S, Grutters J, Joore MA. Development and Validation of the TRansparent Uncertainty ASsessmenT (TRUST) Tool for Assessing Uncertainties in Health Economic Decision Models. Pharmacoeconomics. 2019 Nov 11. doi: 10.1007/s40273-019-00855-9. [Epub ahead of print] 
Pouwels XGLV, Petersohn S, Carrera VH, Denniston AK, Chalker A, Raatz H, Armstrong N, Shah D, Witlox W, Worthy G, Noake C, Riemsma R, Kleijnen J, Joore MA. Fluocinolone Acetonide Intravitreal Implant for Treating Recurrent Non-infectious Uveitis: An Evidence Review Group Perspective of a NICE Single Technology Appraisal. Pharmacoeconomics. 2019 Nov 8. doi: 10.1007/s40273-019-00851-z. [Epub ahead of print]

Pouwels XGLV, Geurts SME, Ramaekers BLT, Erdkamp F, Vriens BEPJ, Aaldering KNA, van de Wouw AJ, Dercksen MW, Smilde TJ, Peters NAJB, van Riel JM, Pepels MJ, Heijnen-Mommers J, Joore MA, Tjan-Heijnen VCG, de Boer M (2019) The relative effectiveness of eribulin for advanced breast cancer treatment: a study of the southeast Netherlands advanced breast cancer registry. Acta Oncologica. doi: 10.1080/0284186X.2019.1670356.

Grimm SE, Fayter D, Ramaekers BLT, Petersohn S, Riemsma R, Armstrong N, Pouwels X, Witlox W, Noake C, Worthy G, Kleijnen J, Joore MA. Pembrolizumab for Treating Relapsed or Refractory Classical Hodgkin Lymphoma: An Evidence Review Group Perspective of a NICE Single Technology Appraisal. PharmacoEconomics. doi: https://doi.org/10.1007/s40273-019-00792-7.

Pouwels XGLV, Grutters JPC, Bindels J, Ramaekers BLT, Joore MA. Uncertainty and Coverage With Evidence Development: Does Practice Meet Theory? Value in health. 2019. 22(7):799-807. doi: https://doi.org/10.1016/j.jval.2018.11.010.

Ramaekers BLT, Riemsma R, Grimm S, Fayter D, Deshpande S, Armstrong N, Witlox W, Pouwels X, Duffy S, Worthy G, Kleijnen J, Joore MA. Arsenic Trioxide for Treating Acute Promyelocytic Leukaemia: An Evidence Review Group Perspective of a NICE Single Technology Appraisal. Pharmacoeconomics. 2018 Nov 14. doi: 10.1007/s40273018-0738-y. [Epub ahead of print]

Grimm SE, Armstrong N, Ramaekers BLT, Pouwels X, Lang S, Petersohn S, Riemsma R, Worthy G, Stirk L, Ross J, Kleijnen J, Joore MA. Nivolumab for Treating Metastatic or Unresectable Urothelial Cancer: An Evidence Review Group Perspective of a NICE Single Technology Appraisal. Pharmacoeconomics. 2018 Oct 6. doi: 10.1007/s40273018-0723-5. [Epub ahead of print] 
Ramaekers BLT, Wolff RF, Pouwels X, Oosterhoff M, Van Giessen A, Worthy G, Noake C, Armstrong N, Kleijnen J, Joore MA. Ixekizumab for Treating Moderate-to-Severe Plaque Psoriasis: An Evidence Review Group Perspective of a NICE Single Technology Appraisal. Pharmacoeconomics. 2018 Feb 26.

Pouwels XGLV, Wolff R, Ramaekers BLT, Van Giessen A, Lang S, Ryder S, Worthy G, Duffy S, Armstrong N, Kleijnen J, Joore MA. Ticagrelor for Secondary Prevention of Atherothrombotic Events After Myocardial Infarction: An Evidence Review Group Perspective of a NICE Single Technology Appraisal. Pharmacoeconomics. 2018. 36:533-543.

Ramaekers BLT, Wolff R, van Giessen A, Pouwels X, Fayter D, Lang S, Armstrong N, Worthy G, Duffy S, Kleijnen J, Joore MA. Trifluridine-Tipiracil for Previously Treated Metastatic Colorectal Cancer: An Evidence Review Group Perspective of a NICE Single Technology Appraisal. Pharmacoeconomics. 2017.

Pouwels $\mathbf{X G L V}_{\mathbf{L}}$ Ramaekers B, Joore MA. Reviewing the quality, health benefit and value for money of chemotherapy and targeted therapy for metastatic breast cancer. Breast Cancer Research and Treatment. 2017 Oct;165(3):485-498. doi: 10.1007/s10549-017-4374-6.

\section{Non-scientific publications}

Chapter 27: "Assessing costs and value for money of breast cancer care" by Joore $M$, Pouwels X, Ramaekers B in "Breast cancer: Global quality care". Edited by Didier Verhoeven, Cary Kaufman, Robert Mansel, and Sabine Siesling. Oxford University Press. Oxford, England.

Pouwels X, Ramaekers BLT, Joore MA. Treatment-Line Versus Patient-Level Matching: A Case Study in Oncology. Value \& Outcomes Spotlight. January/February 2020. 


\section{Documents informing policy decisions}

Riemsma R, Van Asselt ADI, Witlox W, Huertas Carrera V, Posadzki P, Armstrong N, Ryder S, Ramaekers B, Grimm S, Pouwels X, Duffy S, Joore MA, Kleijnen J. Lenalidomide with rituximab for previously treated follicular lymphoma and marginal zone lymphoma: a Single Technology Assessment. York: Kleijnen Systematic Reviews Ltd, 2019.

Riemsma R, Pouwels X, Petersohn S, Chalker A, Huertas Carrera V, Raatz H et al. Fluocinolone acetonide ocular implant for treating recurrent non-infectious uveitis: a Single Technology Assessment. York: Kleijnen Systematic Reviews Ltd2019.

Westwood M, Ramaekers BLT, Petersohn S, Grimm S, Pouwels X, Witlox W, Fayter D, Armstrong N, Shah D, Worthy G, de Kock S, Joore MA, Kleijnen J. Erenumab for preventing migraine: A Single Technology Assessment. York: Kleijnen Systematic Reviews Ltd, 2018.

Wolff R, Grimm S, Ramaekers B, Fayter D, Armstrong N, Pouwels X, Witlox W, Portegijs P, Matovinovic E, Misso K, Worthy G, Joore M, Kleijnen J. Ixekizumab for treating active psoriatic arthritis following inadequate response to diseasemodifying antirheumatic drugs; A Single Technology Assessment. York: Kleijnen Systematic Reviews Ltd, 2018.

Riemsma R, Ramaekers B, Grimm S, Fayter D, Deshpande S, Portegijs, P, Armstrong N, Witlox W, Pouwels X, Duffy S, Worthy G, Joore M, Kleijnen J. Arsenic trioxide for treating acute promyelocytic leukaemia; A Single Technology Assessment. York: Kleijnen Systematic Reviews Ltd, 2018.

Fayter D, Grimm S, Ramaekers B, Petersohn S, Riemsma R, Armstrong N, Witlox W, Pouwels X, Noake C, Worthy G, Joore M, Kleijnen J. Pembrolizumab for treating relapsed or refractory classical Hodgkin lymphoma; A Single Technology Assessment. York: Kleijnen Systematic Reviews Ltd, 2017.

Armstrong N, Grimm S, Ramaekers BLT, Pouwels X, Lang S, Fayter D, Petersohn S, Riemsma R, Worthy G, Stirk S, Ross J, Joore MA, Kleijnen J. Nivolumab for treating metastatic or unresectable urothelial cancer: a Single Technology Assessment. York: Kleijnen Systematic Reviews Ltd, 2017. 
Wolff R, Pouwels X, Ramaekers BLT, Van Giessen A, Birnie R, Lang S, Ryder S, Worthy G, Duffy S, Armstrong N, Joore MA, Kleijnen J. Ticagrelor for secondary prevention of atherothrombotic events after myocardial infarction: a Single Technology Assessment. York: Kleijnen Systematic Reviews Ltd, 2016.

Armstrong N, Ramaekers BLT, Pouwels X, Zaim R, Wolff RF, Riemsma RR, Wei CY, Worthy G, Misso K, Joore MA, Al M, Kleijnen J. Nivolumab for treating recurrent or metastatic squamous-cell carcinoma of the head and neck after platinum-based chemotherapy: a Single Technology Assessment. York: Kleijnen Systematic Reviews Ltd, 2016.

Fayter D, Ramaekers BLT, Grimm S, Pouwels X, Armstrong N, Ryder S, Garcia S, Portegijs P, Noake C, Riemsma R, Joore MA, Kleijnen J. Obeticholic acid for treating primary biliary cirrhosis: a Single Technology Assessment. York: Kleijnen Systematic Reviews Ltd, 2016.

Riemsma R, Joore MA, Ramaekers BLT, Leadley R, Pouwels $\mathbf{X}$, Van Giessen A, Armstrong N, Wei, C-Y, Worthy G, Misso K, Kleijnen J. Sofosbuvir in combination with velpatasvir for treating chronic hepatitis C: a Single Technology Assessment. York: Kleijnen Systematic Reviews Ltd, 2016.

Wolff R, Ramaekers BLT, Van Giessen A, Pouwels X, Fayter D, Lang S, Armstrong N, Worthy G, Duffy $S$, Joore MA, Kleijnen J. Trifluridine in combination with tipiracil hydrochloride for previously treated metastatic colorectal cancer: a Single Technology Assessment. York: Kleijnen Systematic Reviews Ltd, 2016.

Wolff R, Joore MA, Pouwels X, Oosterhoff M, Ramaekers BLT, Van Giessen A, Wei C-Y, Worthy G, Noake C, Armstrong N, Kleijnen J. Ixekizumab for treating moderate to severe chronic plaque psoriasis: a Single Technology Appraisal. York: Kleijnen Systematic Reviews Ltd, 2016.

Riemsma R, Joore MA, Ramaekers BLT, Van Giessen A, Westwood M, Armstrong N, Pouwels X, Worthy G, Stirk L, Severens JL, Kleijnen J. Sebelipase alfa for treating lysosomal acid lipase deficiency: a Highly Specialised Technology Evaluation. York: Kleijnen Systematic Reviews Ltd, 2015. 
Wolff R, Joore MA, Ramaekers B, Tomini F, Pouwels X, Deshpande S, Portegijs P, Stirk L, Worthy G, Armstrong N, Riemsma R, Severens JL, Kleijnen J. Tolvaptan for treating autosomal dominant polycystic kidney disease: a Single Technology Appraisal. York: Kleijnen Systematic Reviews Ltd, 2015.

\section{Oral presentations}

2019: "Introduction to time to event data and survival analysis" during the workshop: "Survival analysis" for Young-NVTAG, October 10, Utrecht, the Netherlands

2019: "A framework to systematically identify and communicate uncertainty in decision analytic models" during the session "Barriers and Proposed Solutions for Using Value of Information Analysis to Improve Reimbursement Decisions: The Case of Tomosynthesis for Breast Cancer Screening." at the international health economic association (iHEA) conference, July, Basel, Switzerland

2018: "The impact of treatment line matching on covariates balance and cost effectiveness results: A case study in oncology" during the workshop "Confounding by indication - current practices and future applications" at the ISPOR $21^{\text {st }}$ Annual European Congress, November, Barcelona, Spain

2018: "Assessing The Certainty Of Modelled Cost Effectiveness Evidence Using A Framework Based On Cochrane's GRADE Tool" during the organised session "From a Lack of Evidence to Action: How to Assess and Manage Uncertainty in HTA" at the $12^{\text {th }}$ European Conference of the Health Economics Association (EuHEA), July, Maastricht The Netherlands

2017: "Did we EMBRACE eribulin in the Dutch clinical practice?" at the Scientific day of the Department of Internal Medicine, Division of Medical Oncology, School for Oncology and Developmental Biology, Maastricht University Medical Centre+, September 4, Maastricht, The Netherlands 


\section{Poster presentations}

2018 "Patient versus treatment line matching: impact on covariates balance and cost effectiveness results" during the ISPOR $21^{\text {st }}$ Annual European Congress, November, Barcelona, Spain

2018: "Uncertainty and Coverage with Evidence Development: Does Practice Meet Theory?" at the $12^{\text {th }}$ European Conference of the Health Economics Association (EuHEA), July, Maastricht, The Netherlands

2017: "Survival model selection uncertainty in model-based cost-effectiveness analysis" during the ISPOR $20^{\text {th }}$ Annual European Congress, November, Glasgow, Scotland

2016: "Missed opportunities: the negligence of non-statistical uncertainty in the Dutch CED policy" during the ISPOR $19^{\text {th }}$ Annual European Congress, November, Vienna, Austria

2015: "Resource use and cost of chemotherapeutic treatment for metastatic breast cancer in the Netherlands" during the ISPOR $18^{\text {th }}$ Annual European Congress, 7-11 November, Milan, Italy

2015: "Reviewing the cost-effectiveness of chemotherapy and targeted therapy for metastatic breast cancer" during the ISPOR $18^{\text {th }}$ Annual European Congress, 7-11 November, Milan, Italy 
Addenda 
Dankwoord 
Addenda 
Voilà, c'est fini, le livre est enfin là ...! Mais arriver à ce résultat n'aurait pas été possible sans la contribution et le soutient de certaines personnes! Comme vous le savez, je peux être un homme de peu de mots, donc si tu n'es pas mentionné dans ce mot de remercîment, cela ne veut pas dire que je ne t'ai pas dans mon cœur.

Zonder de hulp en steun van meerdere personen was dit proefschrift niet geworden wat het geworden is... Daarom wil ik graag de mensen bedanken die hieraan hebben meegewerkt. Daarnaast wil ik mensen uit mijn persoonlijke omgeving bedanken die (af en toe zonder het te weten) de nodige zet in de rug en afleiding hebben gegeven. Zoals jullie weten, kan ik een man van weinig woorden zijn, dus als jij hier niet benoemd wordt, betekent het niet dat ik je niet in mijn hart draag.

Allereerst wil ik graag mijn promotieteam bedanken. Manuela, bedankt voor jouw kritische blik op mijn werk. Je hebt mij gedurende 4,5 jaar bij heel veel verschillende projecten betrokken waardoor ik meerdere kanten van economische evaluaties en van het besluitproces heb kunnen ervaren. Ik ben heel dankbaar dat je me die mogelijkheden hebt gegeven. Verder heb je nooit nee gezegd tegen congresbezoeken die allemaal leerzaam en gezellig waren. Bram, bedankt voor het stellen van de goede en moeilijke vragen. Zelfs als ik af en toe je kantoor uitkwam met nog meer vragen dan toen ik binnenkwam, heb je mij geleerd om kritisch na te denken over welke keuzen ik maak(te). Kennis van methoden is natuurlijk niet het enige waarop jij mij bleef verbazen! Door jouw brede interesse in technologische innovaties die je zo enthousiast met mij deelde, had ik het gevoel dat ik niet helemaal onder een steen leefde. Verder, het was heel fijn dat je kantoordeur altijd open stond zodat ik snel vraagjes kon komen stellen. Maaike, je sloot later aan bij de team maar jij gaf de nodige klinische blik om sommige drogere economische stukken wat interessanter te maken voor het publiek die we in het oog hadden. Ik vond het heel fijn dat je altijd navroeg of je niet teveel vroeg als we besloten analyses anders uit te voeren of om extra analyses te draaien. Ik ben nog steeds verbaasd dat je tijd vond (en nog steeds vindt) naast patiëntenzorg om al onze afspraken goed voor te bereiden.

Daarnaast wil ik graag de beoordelingscommissie bedanken voor het doornemen en beoordelen van dit boek, en voor jullie virtuele of fysieke aanwezigheid in Maastricht! (Op het moment dat ik dit schreef was het dus onduidelijk wat er wel/ niet mogelijk zou zijn op 27 November) 
I would also like to thank all the co-authors on the papers included in this thesis. Sabine, it has been a pleasure to work with you. You're always enthusiastic and thorough in your work which is really inspiring! En Janneke, het was heel fijn om met jou te kunnen samenwerken tijdens deze periode. Alhoewel je heel druk bent, bleef je vragen of je op de een of andere manier mij kon ondersteunen, en dat waardeer ik heel erg.

KEMTA - Andrea, Ben, Bram, Brigitte, Carmen, Daisy, Hassan, Irene, Julie, Lloyd, Loeki, Maarten, Manuela, Marije, Martijn, Merel, Monique, Sabine, Sander, Sophie, Stella, Svenja, Willem, Yvonne - bedankt voor de fijne tijd. Ik wil vooral de jongere onderzoekers van KEMTA bedanken, het was heel gezellig om ook na werk samen een drankje te kunnen doen! Verder heb ik een aantal kamergenoten gehad die van dichterbij mijn traject meegemaakt hebben. Alles begon met Adriënne in ons kamertje met uitzicht op de parking aan het eind van de gang. Als rookies, hebben we samen ontdekt hoe het leven bij KEMTA ging. Het was heel fijn om dat kamertje met jou te delen. Het werk ging voor, maar dit nam niet weg dat we altijd een luisterend hoor hadden voor elkaar, wat best belangrijk was tijdens deze stageperiode. After that year, I've started to share a room with Stella and Svenja. I was not the rookie anymore but could help other rookies to find their ways. Even though I played the serious guy sometimes, we had a lot of fun together. Of course Svenja, you've got a special place here since we also lived together a year, but we did not mix private matters and work, which was really nice (you got this one right?). It was good to be able to share the good stuffs of life but also the frustrations (no need to be extensive here...) with you without being judged. And we're still in touch through Jong-NVTAG, so who knows when this will end? Thanks for introducing me to the veggie life as well! Stella, jij bent degene die het het langst volgehouden heeft met mij op kantoor. Ik denk dat er in de loop van de tijd er steeds minder taboes waren tussen ons, en dat was gewoon fijn want het zorgde dat we allebei af en toe chagrijnig of heel blij konden zijn. Daarna kwam Dorthe het plekje van Svenja overnemen en introduceerde iets in mijn leven waar ik nog helemaal niet mee bezig was... Ik moet toegeven dat ik hieraan moest wennen, maar nu snap ik helemaal dat een huis kopen constant in je hoofd blijft spelen... Bedankt dat je wat extra leven in het kantoortje kwam blazen! Toen kwam Willem er ook eventjes ook bijzitten. Ja, wat kan ik zeggen...? Bedankt voor de visverhalen en het vissen! Je vrolijkheid is niet te overtreffen, dus bedankt voor de humor die je elke dag meebracht naar kantoor. En uiteindelijk, Sophie, jij kwam bij ons zitten aan het eind van mijn tijd bij KEMTA. Jij straalt rust uit en jij bent altijd enthousiast over een goede gesprek. Dus bedankt voor deze momenten! 
Naast de jongere onderzoekers van KEMTA wil ik ook Brigitte, Merel, en Sander bedanken voor de leuke discussie tijdens onze koffiemomenten.

I would also like to thank the whole NICE team - Anoukh, Ben, Bram, Manuela, Sabine, Svenja, Théa, Willem. It was nice to work together besides performing more individual research. I have learned a lot from these assessments and from working as a member of the team. We were bonded and had a sort of agreement that, sometimes, the team goes first above the individual. I am grateful for that since it allowed to provide high quality assessments which were finished within our time line. I would also like to thank our colleagues from Kleijnen Systematic Reviews for the pleasant collaborations on these assessments.

Ik wil ook graag de ISPOR Student Chapter van Maastricht bedanken met wie we mooie activiteiten hebben georganiseerd maar vooral hebben laten zien dat pubquizz echt ons ding is... Bedankt Ben, Inge, Ingrid, Irina, Kei-Long, Luka, Marije, Svenja, en Stella!

My time in Maastricht would not have been the same without ULTIMAAS, my beloved ultimate frisbee team. Even though I left through the back door, thank you guys and girls for the nice training sessions, nights out, and tournaments!

Les amis, merci pour toutes les distractions durant nos plus ou moins long weekends! Thomas, c'est cool d'avoir pu démolir l'intérieur de ta maison pour évacuer mes frustrations!

Mes dernier mots vont à mon entourage. Maman, merci pour tout! Même si ce que je fais dans la vie reste un peu obscure pour toi, ça ne t'a pas empêché de me pousser à finir ce que j'ai commencé. Si je suis là aujourd'hui, c'est grâce à toi. Il serait aussi injuste de passer mon papa. Même si nous avons nos différents, tu fais partie de ma vie et a contribué à la personne que je suis aujourd'hui. Tout comme bon papa, bonne ma, et mamy, et tous mes oncles et tantes. Les bros, Ro et Jo, vous êtes deux cowboys comparé à moi. On a fait des choix différents : j'ai choisi une certaine facilité et stabilité alors que vous avez choisis un chemin plus risqué dans vos vies professionnelles. Vous me montrez chaque jour que la vie peut être approchée différemment et que ce n'est pas la stabilité qui rend heureux mais ce que tu fais de ta journée. Merci pour ces leçons de vie!

Teun, ja hoor, je valt ook onder familie! Hoeveel nachten in Rotterdam heb je mij ondervraagd over mijn werk, wat mij blij maakt, wat belangrijk voor mij is ...? Ik kan 
me het niet herinneren want deze nachten werden af en toe ook een beetje wazig... Die vragen maakten mij ongemakkelijk maar ik leerde er van. We zien elkaar nog steeds, minder regelmatig dan wat ik zou willen, maar dat hoeft niet want we zijn er voor elkaar geweest en zullen er voor elkaar zijn in de toekomst. Ik ben je dankbaar dat je zo'n goede vriend bent!

Mijn schatje, jij bent er geweest tijdens de leuke en minder leuke momenten. Net als mama, heb je mij bijna achter mijn scherm gezet om dit af te ronden. Dus bedankt voor de gezelligheid en discipline thuis, de rest weet je wel! 
Author information 
Addenda 


\section{Author information}

Xavier was born on Augusts $17^{\text {th }} 1991$ in Verviers, Belgium. Xavier grew up in Belgium and after his secondary education, he moved to Diever, the Netherlands, where he spent one academic year refining his Dutch in a guest family. After this experience 'abroad', Xavier started the Health Sciences Bachelor (Biology-track) at Maastricht University in 2010. He then enrolled in 2013 for the Health Sciences Research Master, still at Maastricht University, during which he followed the PREMIUM

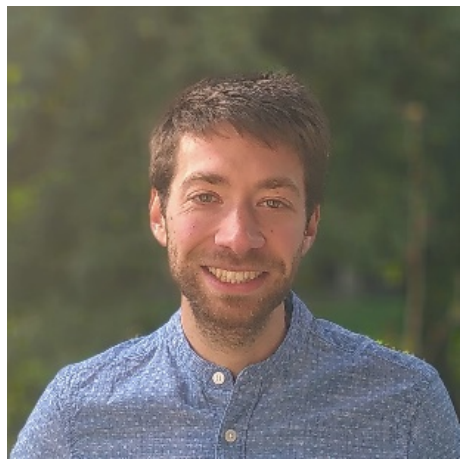
Honours Program. After graduating, Xavier started his PhD research in 2015 at the department of Clinical Epidemiology and Medical Technology Assessment (KEMTA) from the Maastricht University Medical Centre+ (MUMC+). During his doctoral research, Xavier was a member of an evidence review group, in collaboration with Kleijnen Systematic Reviews, for the National Institute for Health and Care Excellence (NICE) of the United Kingdom. Xavier was also active in teaching activities. He was a tutor in the Healthcare Policy, Innovations, and Management Master program and a lecturer and practical supervisor in the Health Sciences Research Master. During his $\mathrm{PhD}$ research, Xavier participated in multiple committees, such as the International Society for Pharmacoeconomics and Outcome Research (ISPOR) Student Chapter of Maastricht University, the CAPHRI PhD panel, and he received a distinguished service award from ISPOR due to his contribution to the society. Xavier is currently president of the Jong-NVTAG committee. Since February 2020, Xavier is working as a post-doc researcher at the department of Health Technology and Services Research at the University of Twente. 
